\title{
Radioactive Waste Management Information for 1994 and Record-To-Date
}
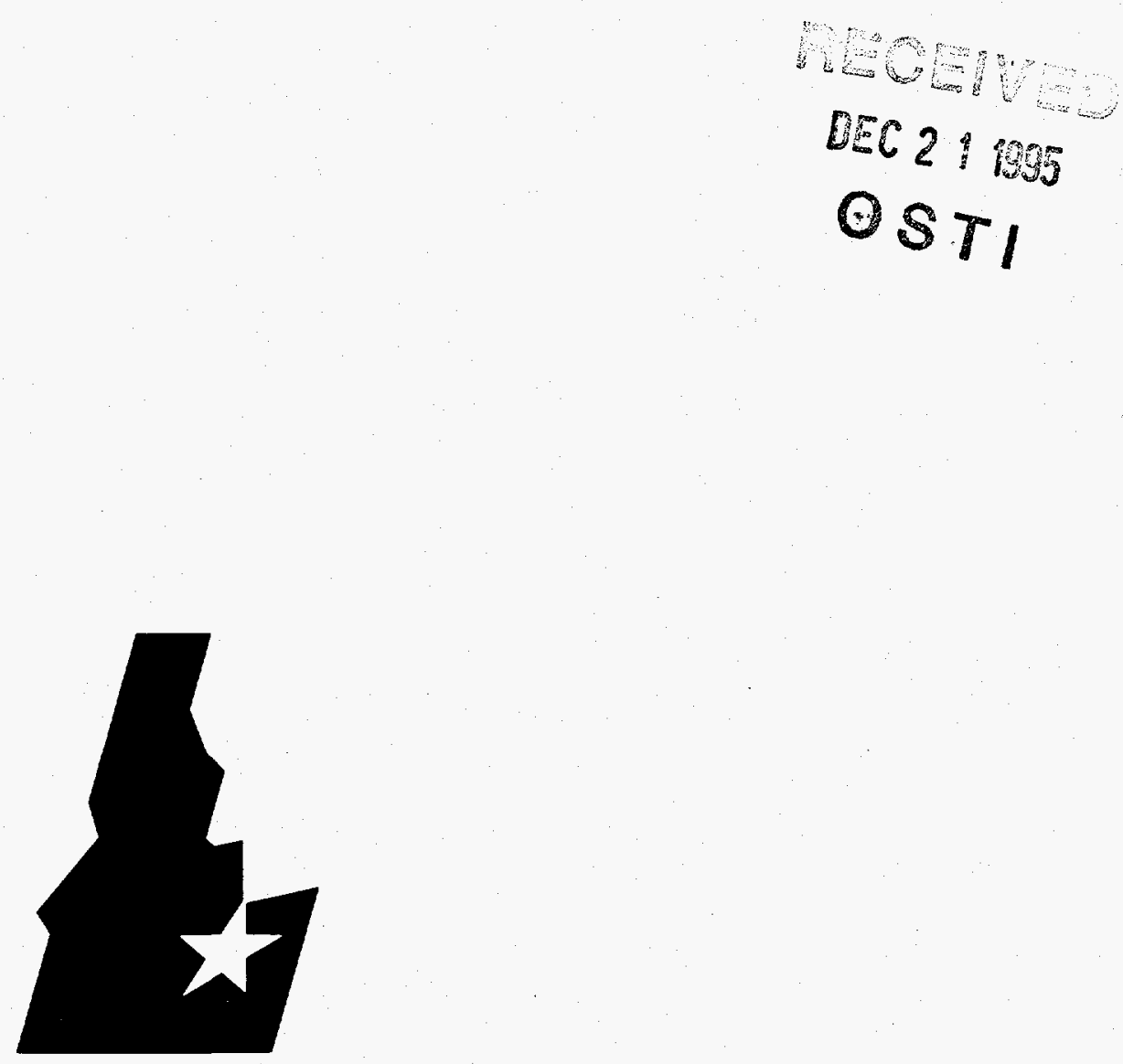

Idaho National Engineering Laboratory

U.S. Department of Energy $\cdot$ Idaho Operations Office
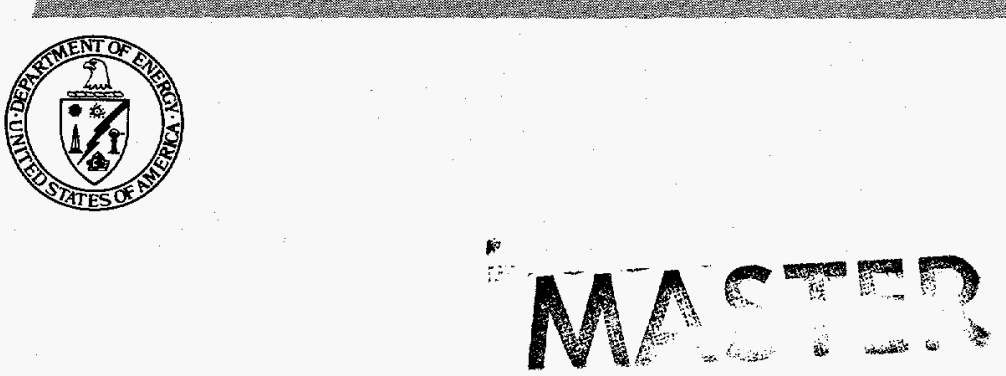


\section{DISCLAMIER}

Portions of this document may be illegible in electronic image products. Images are produced from the best available original document. 
DOE/ID-10054(94)

Distribution Category

Limited

\title{
Radioactive Waste Management Information For 1994 And Record-To-Date
}

\author{
D.L. French \\ D.J. Lisee \\ K.A. Taylor
}

Environmental Operations, Branch Support

Published July 1995

Idaho National Engineering Laboratory Lockheed Martin Idaho Technologies Idaho Falls, Idaho $\mathbf{8 3 4 1 5}$

Prepared for the U.S. Department of Energy

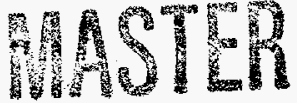
DOE Idaho Operations Office Contract No. DE-AC07-94ID 13223 



\section{ABSTRACT}

This document presents detailed data, bar graphs, and pie charts on volume, radioactivity, isotopic identity, origin, and decay status of radioactive waste for calendar year 1994. It also summarizes the radioactive waste data records compiled from 1952 to present for the Idaho National Engineering Laboratory (INEL). The data presented are from the INEL Radioactive Waste Management Information System.

\section{DISCLAIMER}

This report was prepared as an account of work sponsored by an agency of the United States Government. Neither the United States Government nor any agency thereof, nor any of their employees, makes any warranty, express or implied, or assumes any legal liability or responsibility for the accuracy, completeness, or usefulness of any information, apparatus, product, or process disclosed, or represents that its use would not infringe privately owned rights. Reference herein to any specific commercial product, process, or service by trade name, trademark, manufacturer, or otherwise does not necessarily constitute or imply its endorsement, recommendation, or favoring by the United States Government or any agency thereof. The views and opinions of authors expressed herein do not necessarily state or reflect those of the United States Government or any agency thereof. 


\section{EXECUTIVE SUMMARY}

This document, Radioactive Waste Management Information for 1994 and Record-To-Date, contains computerized radioactive waste data records from the Idaho National Engineering Laboratory (INEL). Data are compiled from information supplied by the U.S. Department of Energy (DOE) contractors. Data listed are on airborne and liquid radioactive effluents and solid radioactive waste that is stored, disposed, and sent to the INEL for reduction. Data are summarized for the years 1952 through 1993. Data are detailed for the calendar year 1994.

Airborne and liquid waste was released to the environment through engineered release points identified on Table 1 located in the Engineered Release Points section. Monitoring and effluent sampling systems are an integral part of each engineered release point. Releases to the environment may also occur at locations other than normal release points.

Airborne releases of radioactivity for 1994 were 2,230 curies; noble gases accounted for 2,184 curies or $98 \%$ of the radioactivity. The curie amount compares to 2,719 curies in 1993 and 24,179 curies in 1992.

A total of $1.995 \mathrm{E}+09$ liters of liquid radioactive waste containing 50 curies was released to the environment at the INEL during 1994. Tritium accounted for $94 \%$ of the radioactivity. As a result of radioactive decay, 47 curies or 94\% of the curies for identified radionuclides remained on January 1, 1995, and 26 curies or $52 \%$ will remain after 5 years. The radioactivity discharged as liquid waste in 1994 was $62 \%$ less than the activity discharged in 1993 and 73\% less than the activity discharged in 1992.

During 1994, a total of $1,906 \mathrm{~m}^{3}$ of solid radioactive waste containing 49,524 curies was shipped to the Radioactive Waste Management Complex (RWMC) for disposal. This compares to $852 \mathrm{~m}^{3}$ with 429,655 curies disposed in 1993 , and $844 \mathrm{~m}^{3}$ with 143,951 curies in 1992. By January 1,1995 , the radionuclides identified in 1994 had decayed to 44,707 curies $(90 \%)$, and in 5 years they will have decayed to 32,970 curies $(67 \%)$.

The majority of the nuclides disposed in solid waste at RWMC in 1994 consisted of $\mathrm{Ni}-63$, $\mathrm{Co}-60$, and $\mathrm{Fe}-55$. These nuclides accounted for $92 \%$ of total curies.

Two cubic meters containing 49 curies of TRA waste initially received in 1990 was moved to a new storage location on Intermediate-Level Transuranic Storage Facility (ILTSF). An error was made on the location date that allowed it to roll into the 1994 TRA ILTSF stored waste total. The error was corrected, but was too late to appear in the data roll-up for this report.

The Waste Calcining Facility did not operate in 1994.

The Waste Experimental Reduction Facility (WERF) received $769 \mathrm{~m}^{3}$ and 1 curie of solid radioactive waste for size reduction in 1994. The waste received by WERF for reduction in 1994 compared to $446 \mathrm{~m}^{3}$ and 2 curies in 1993 and $940 \mathrm{~m}^{3}$ and 13 curies in 1992. Because of reevaluation of the shipments to WERF the 1991 and 1992 totals will be different.

In addition to the waste sent to WERF for volume reduction, $1,713 \mathrm{~m}^{3}$ containing 26 curies were sent off-site to Scientific Ecology Group. The ash was returned to RWMC for disposal.

Radioactive effluent discharges at the INEL in 1994 met all applicable DOE requirements (DOE 5400.5, 5820.2) and State of Idaho regulations and standards.

The impact of this waste on the environment, both onsite and offsite, is constantly monitored 
by the INEL Site Environmental Surveillance Program. The results of this program for 1994 are published in the annual report, The Idaho National Engineering Laboratory Site Environmental Report for Calendar Year 1994, DOE/ID-12082(94), published July 1994.

The effect of radioactive decay reduces quantities of activity at varying rates among the radionuclides. The effect of decay on airborne, liquid, and solid radioactive waste disposed on the INEL in 1994 is in INEL 1994 Summaries section. The decay summary data for the INEL for the period of 1952-1994 is found in the Area Decay Summaries section. Only curies of identified radionuclides have decayed. Although all radioactivity decays, decay schemes cannot be applied to the curies of unidentified radionuclides such as mixed fission or activation products. 


\section{CONTENTS}

ABSTRACT $\ldots \ldots \ldots \ldots \ldots \ldots \ldots \ldots \ldots \ldots \ldots \ldots \ldots \ldots \ldots \ldots \ldots \ldots$ iii

EXECUTIVE SUMMARY $\ldots \ldots \ldots \ldots \ldots \ldots \ldots \ldots \ldots \ldots \ldots \ldots$

ACRONYMS AND ABBREVIATIONS $\ldots \ldots \ldots \ldots \ldots \ldots \ldots \ldots \ldots \ldots \ldots \ldots \ldots$ ix

CONVERSION FACTORS $\ldots \ldots \ldots \ldots \ldots \ldots \ldots \ldots \ldots \ldots \ldots \ldots \ldots \ldots \ldots \ldots \ldots$ xii

DEFINITIONS $\ldots \ldots \ldots \ldots \ldots \ldots \ldots \ldots \ldots \ldots \ldots \ldots \ldots \ldots \ldots \ldots \ldots \ldots \ldots \ldots \ldots$

INTRODUCTION $\ldots \ldots \ldots \ldots \ldots \ldots \ldots \ldots \ldots \ldots \ldots \ldots \ldots \ldots \ldots \ldots \ldots$

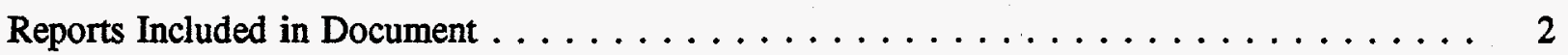

\section{TAB SECTIONS}

INEL Record-to-Date Summary

INEL Record-to-Date Discharges and Solid Waste

INEL Record-to-Date Decay Summaries

Area Record-to-Date Decay Summaries

INEL 1994 Year-to-Date Summary

INEL Disposed Waste Decay Summary in Curies for 1994

Nuclide Summary in Curies for Airborne, Liquid, and Solid Waste

Argonne National Laboratory-West 1994 Graphics

Central Facilities Area 1994 Graphics

Chemical Processing Plan 1994 Graphics

Decontamination and Decommissioning 1994 Graphics

Naval Reactor Facility 1994 Graphics

Power Burst Facility 1994 Graphics

Radioactive Waste Management Complex 1994 Graphics

Special Manufacturing Capability 1994 Graphics

Special Power Excursion Reactor 1994 Graphics

Test Area North 1994 Graphics

Test Reactor Area 1994 Graphics

Waste Experimental Reduction Facility 1994 Graphics

Engineered Release Points 


\section{ACRONYMS AND ABBREVIATIONS}

A1W Large ship reactor at Naval Reactors Facility

ALE Argonne National Laboratory-East

ANL Argonne National Laboratory-West

ARA Auxiliary Reactor Area

ATR Advanced Test Reactor

BCL Battelle Columbus Laboratories

BEN Bendix - Grand Junction, Colorado

BET Bettis Atomic Power Laboratory

BNL Brookhaven National Laboratory

B\&W Babcock \& Wilcox

CEG Combustion Engineering General Atomics

CFA Central Facilities Area

CG Concentration Guide (also referred to as DCG)

CPP Chemical Processing Plant (also referred to as ICPP)

CTF Containment Test Facility (LOFT became CTF on 10/01/86)

CY calendar year

D\&D decontamination and decommissioning

DCG Derived Concentration Guide

DOE U.S. Department of Energy

EBR-II Experimental Breeder Reactor II

ECF Expended Core Facility

EG\&G EG\&G, Incorporated

ETR Engineering Test Reactor

FASB Fuel Assembly and Storage Building

FAST Fluorinel and Storage Facility

FCF Fuel Cycle Facility

FMF Fuel Manufacturing Facility

JCH J.C. Haynes/Wright Patterson Air Force Base

HFEF Hot Fuels Examination Facility
HFEF-N Hot Fuels Examination FacilityNorth

HFEF-S Hot Fuels Examination FacilitySouth

ICPP Idaho Chemical Processing Plan (also referred to as CPP)

ILTSF Intermediate-Level Transuranic Storage Facility

INEL Idaho National Engineering Laboratory

L\&O Lab and Office Building

LOF Loss-of-Fluid Test

LOFT Loss-of-Fluid Test

MAP Mixed Activation Products

MDL Monsanto Dayton Laboratory

MFP Mixed Fission Products

MRC Monsanto Research Corporation Mound Laboratory

MTR Materials Test Reactor

MWSF Mixed Waste Storage Facility

NRF Naval Reactors Facility

NSA North Storage Area

NWCF New Waste Calcining Facility

OFF offsite

OTH other

PBF Power Burst Facilty

PER Special Power Excursion Reactor Test (also referred to as SPERT)

PREPP Process Reduction Experimental Pilot Plant

R\&D research and development

RAL Radiation Analysis Laboratory

RC Reactor Compartment (NRF only)

RESL/IL Radiological and Environmental Sciences Laboratory/Idaho Operations 
RFO Rocky Flats Office (EG\&G Rocky Flats, Colorado)

RLWTF Radioactive Liquid Waste Treatment Facility

RWDS Radioactive Waste Disposal System

RWMC Radioactive Waste Management Complex

RWMIS Radioactive Waste Management Information System

S1W submarine prototype at NRF

S5G submarine prototype at NRF

SCND Special Case Nondefense Waste

SDA Subsurface Disposal Area

SEG Scientific Ecology Group

SL-1 Stationary Low Power Reactor No. 1

SMC Specific Manufacturing Capability

SPERT Special Power Excursion Reactor Test (also referred to as PER)

SPF Sodium Process Facility
SWEPP Stored Waste Examination Pilot Plant

TAN Test Area North

TRA Test Reactor Area

TREAT Transient Reactor Test Facility

TSA Transuranic Storage Area

TSF Test Support Facility

WCF Waste Calcining Facility

WER Waste Experimental Reduction Facility (also referred to as WERF)

WERF Waste Experimental Reduction Facility (also referred to as WER)

WINCO Westinghouse Idaho Nuclear Company

WMC Waste Management Complex (also referred to as RWMC)

WVRF Waste Volume Reduction Facility

ZPPR Zero Power Plutonium Reactor 


\section{CONVERSION FACTORS}

\begin{tabular}{llr} 
To Convert & \multicolumn{1}{c}{ Into } & Multiply By \\
\cline { 2 - 3 } Cubic yards & Cubic meters & 0.7646 \\
Cubic meters & Cubic feet & 35.5140 \\
Liters & Gallons (U.S. liquid) & 0.2642 \\
Kilograms & Pounds & 2.2046
\end{tabular}

\section{DEFINITIONS}

Curie A unit of radioactivity, defined as that quantity of any radioactive nuclide that has $3.7 \mathrm{E}+10$ disintegrations per second (abbreviated $\mathrm{Ci}$ ).

Exponential notation Numbers in this document are expressed as either whole numbers or in exponential notation form. For example: $1,000,000=1 \times 10^{6}=1 \mathrm{E}+06$. 


\section{RADIOACTIVE WASTE MANAGEMENT INFORMATION FOR 1994 AND RECORD-TO-DATE}

\section{INTRODUCTION}

This document summarizes radioactive waste data records for the Idaho National Engineering Laboratory (INEL) compiled since 1952, and provides detailed data for calendar year (CY) 1994. A computerized Radioactive Waste Management Information System (RWMIS) has been used at the INEL since January 1971.

Several improvements to the data base system and data reporting have taken place since data collection was computerized. These improvements include:

- In 1982, the data base language was converted from COBOL to NOMAD, a fourth generation data base language. NOMAD has many statistical analysis features that can be applied to the data, and allows for modifying data reporting requirements with minimal impact on the data base.

- In January 1986 , solid waste data reporting requirements changed from a per-shipment basis to a per-container basis. A shipment could consist of 1 to 70 containers. By changing to a per-container basis, solid waste on the INEL is tracked more accurately. it is now possible to pinpoint the exact location of a container and to determine its waste classification.

- In February 1988, waste generators were given the ability to provide the analytical uncertainty, at one sigma, for the reported nuclides of air and liquid effluent releases.

U.S. Department of Energy (DOE) contractors routinely report information on airborne and liquid radioactive effluents and solid radioactive waste that is stored, disposed, and sent to the
INEL for reduction. Types of information include volume, radioactivity, isotopic identity, origin, and decay status. This system serves as the official repository for this data and provides reports for all types of radioactive effluents and solid waste disposed, sent for reduction, or stored at the INEL.

Graphics were added to the report in 1987 to improve the data presentation. In the Decay Summaries section, those ares having no identified nuclides, or only $<1$ curie of identified nuclides, are not presented with graphics. The area-specific solid low-level waste graphs in this report depict waste in five categories:

1. Direct Disposal-Waste that is sent directly to the Radioactive Waste Management Complex for disposal with no volume reduction efforts applied.

2. Compaction-Compactible waste that is sent to the Waste Experimental Reduction Facility (WERF) to be compacted.

3. Metal Sizing-Metallic waste that is sent to WERF for volume reduction.

4. Incineration-Combustible waste sent to WERF for incineration.

5. Waste shipped to private industry for volume reduction.

In 1990, additional reports and graphics were added to better represent previously summarized data. These reports and graphics included a detailed list of generators who ship waste to WERF and details on stored waste by generator. 
The RWMIS is continually undergoing review and enhancement. Comments on the system are encouraged. This report is updated annually to incorporate waste management data for the current year and to reflect changes from previous annual reports. These changes are made to more accurately reflect the current status of waste operations at the INEL.

Annual and special RWMIS reports have been useful to various levels of management in appraising their radioactive waste programs. Annual reports provide a summary by type and producer of waste. Special reports include radioactive decay from time of disposal to specified future dates. The volume, weight, and curie content in all RWMIS reports are expressed in exponential notation.

A comparison of the annual mean average released nuclides concentrations and the Derived Concentrations Guide (DCG) limits are included in the detailed RWMIS. The concentration of liquid releases is compared directly to the reference DCG limits for drinking water. It must be noted that the DCG liquid release limits are for protecting the public from ingesting radiationcontaminated water. INEL liquid releases flow to areas inaccessible to the general public. The calculated concentration of airborne releases as dispersed to the INEL boundary is compared to the reference airborne DCG limits. The reference DCG values are given in DOE Order 5400.5, "Radiation Protection of the Public and the Environment," February 1990. The righthand column of many RWMIS reports indicate total and average values. Total values include the total content in curies for each radionuclide released during the year. Average values are the annual mean concentrations of radionuclides.

The RWMIS provides readily available information that permits ongoing evaluation of INEL waste management activities, including compliance with DOE regulations and those of other Federal and State agencies. The TWMIS continues to be a valuable asset to the overall manage- ment effort at the INEL and continues to alert the DOE Idaho Operations Office (DOE-ID) of trends and potential problem areas.

\section{Reports Included in Document}

Eight report categories are included in this document. A brief description of each are below.

INEL Record-to-Date Summary-This report summarizes the volume and curies of all effluents and solid waste on the INEL by CY for the period 1985 to 1994 and a cumulative total for 1952 to 1984.

INEL Record-to-Date Discharges and Solid Waste-These reports summarize the volume and curies of all discharges and solid waste on the INEL by discharge/waste type for each area generating waste by CY for the period 1985 to 1994 and a cumulative total for 1952 to 1984.

INEL Record-to-Date Decay SummariesThese reports summarize both identified and unidentified curies by waste type on the INEL by CY for the period 1985 to 1994 and a cumulative total for 1962 to 1984 . The curie amount is adjusted to allow for radioactive decay through the last day of the current year.

\section{Area Record-to-Date Decay Summaries -} These reports summarize the identified and unidentified curies by area and waste type on the INEL by CY for the period 1985 to 1994 and a cumulative total for 1962 to 1984 . The curie amount is adjusted to allow for radioactive decay through the last day of the current year and then 10 years later.

INEL 1994 Vear-to-Date Summary-This report summarizes, by facility, the amounts of radioactivity generated at INEL facilities or at offsite facilities and shipped to the INEL. Liquid and airborne waste that is released is identified, and quantities of solid wastes stored, 
disposed, or received for reduction are given. The volume of the media containing the radioactivity is also included.

\section{INEL Disposed Waste Decay Summary in} Curies for 1994-This report summarizes the total curies of airborne, liquid, and solid waste on the INEL for the current year and then corrects the amount of the total curies for radioactive decay, by year, for 7 years.
Nuclide Summary in Curies for Airborne, Liquid, and Solid Waste-These reports summarize the annual curie values, by nuclide, for each area reporting effluents or solid waste on the INEL.

Area 1994 Graphics-These graphics include volume and curie monthly data for airborne, liquid, and solid waste generated by INEL areas and for non-INEL areas shipping solid waste to the INEL. 
INEL Record-to-Date Summary

INEL Record-to-Date Summary $\ldots \ldots \ldots \ldots \ldots \ldots \ldots \ldots \ldots \ldots \ldots \ldots \ldots$ INEL-3

INEL Record-to-Date Summary Bar Graphs $\ldots \ldots \ldots \ldots \ldots \ldots \ldots$ INEL-4 



\begin{tabular}{|c|c|c|c|c|c|c|c|c|c|c|c|c|}
\hline TYPE/YEAR & $1952-1984$ & 1985 & 1986 & 1987 & 1988 & 1989 & 1990 & 1991 & 1992 & 1993 & 1994 & TOTAL \\
\hline \multicolumn{13}{|l|}{ AIRBORNE } \\
\hline $\begin{array}{l}\text { VOLUME } \\
\text { CURIES }\end{array}$ & $\begin{array}{r}140664 \\
13050065\end{array}$ & $\begin{array}{r}9636 \\
77262\end{array}$ & $\begin{array}{l}12289 \\
14518\end{array}$ & $\begin{array}{r}12220 \\
254948\end{array}$ & $\begin{array}{r}13388 \\
173935\end{array}$ & $\begin{array}{l}13514 \\
22370\end{array}$ & $\begin{array}{l}13450 \\
24459\end{array}$ & $\begin{array}{l}12963 \\
63879\end{array}$ & $\begin{array}{l}12715 \\
24179\end{array}$ & $\begin{array}{r}13150 \\
2719\end{array}$ & $\begin{array}{r}12856 \\
2230\end{array}$ & $\begin{array}{r}266845 \\
13710563\end{array}$ \\
\hline \multicolumn{13}{|l|}{ LIQUID } \\
\hline $\begin{array}{l}\text { VOLUME } \\
\text { CURIES }\end{array}$ & $\begin{array}{l}72243 \\
73938\end{array}$ & $\begin{array}{r}2744 \\
667\end{array}$ & $\begin{array}{r}2529 \\
346\end{array}$ & $\begin{array}{r}2536 \\
357\end{array}$ & $\begin{array}{r}2357 \\
270\end{array}$ & $\begin{array}{r}1922 \\
137\end{array}$ & $\begin{array}{r}2642 \\
189\end{array}$ & $\begin{array}{r}2490 \\
170\end{array}$ & $\begin{array}{r}2799 \\
187\end{array}$ & $\begin{array}{r}2659 \\
130\end{array}$ & $\begin{array}{r}1995 \\
50\end{array}$ & $\begin{array}{l}96915 \\
76441\end{array}$ \\
\hline \multicolumn{13}{|c|}{ SOLID DISPOSED } \\
\hline $\begin{array}{l}\text { VOLUME } \\
\text { CURIES }\end{array}$ & $\begin{array}{r}191510 \\
10199026\end{array}$ & $\begin{array}{r}3140 \\
271435\end{array}$ & $\begin{array}{r}3437 \\
95084\end{array}$ & $\begin{array}{r}2969 \\
247521\end{array}$ & $\begin{array}{r}2045 \\
149742\end{array}$ & $\begin{array}{r}1364 \\
590066\end{array}$ & $\begin{array}{r}1762 \\
207543\end{array}$ & $\begin{array}{r}1272 \\
187568\end{array}$ & $\begin{array}{r}844 \\
143951\end{array}$ & $\begin{array}{r}852 \\
429655\end{array}$ & $\begin{array}{r}1906 \\
49524\end{array}$ & $\begin{array}{r}211102 \\
12571115\end{array}$ \\
\hline \multicolumn{13}{|l|}{ SOLID STORED } \\
\hline $\begin{array}{l}\text { VOLUME } \\
\text { CURIES }\end{array}$ & $\begin{array}{r}57247 \\
83252352\end{array}$ & $\begin{array}{r}3376 \\
55521\end{array}$ & $\begin{array}{r}3128 \\
55109\end{array}$ & $\begin{array}{r}2217 \\
3400798\end{array}$ & $\begin{array}{r}1395 \\
8609486\end{array}$ & $\begin{array}{r}880 \\
51118\end{array}$ & $\begin{array}{r}41 \\
4607\end{array}$ & $\begin{array}{r}178 \\
1603000\end{array}$ & 39741 & $\begin{array}{r}203 \\
1142363\end{array}$ & $\begin{array}{r}6 \\
72663\end{array}$ & $\begin{array}{r}68670 \\
98286758\end{array}$ \\
\hline $\begin{array}{c}\text { SOLID TO SEE } \\
\text { VOLUAY } \\
\text { CURIES }\end{array}$ & FOR REDUCT & & & & & & & & & & $\begin{array}{r}1713 \\
26\end{array}$ & $\begin{array}{r}1713 \\
26\end{array}$ \\
\hline $\begin{array}{l}\text { SOLID TO WER } \\
\text { VOLUME } \\
\text { CURIES }\end{array}$ & $\begin{array}{c}\text { F FOR REDUC } \\
<18\end{array}$ & $\begin{array}{r}1275 \\
1\end{array}$ & $\begin{array}{r}2458 \\
2\end{array}$ & $\begin{array}{r}2529 \\
5\end{array}$ & $\begin{array}{r}2967 \\
6\end{array}$ & $\begin{array}{r}2663 \\
6\end{array}$ & $\begin{array}{r}3161 \\
12\end{array}$ & $\begin{array}{r}2029 \\
15\end{array}$ & $\begin{array}{r}940 \\
13\end{array}$ & $\begin{array}{r}446 \\
2\end{array}$ & $\begin{array}{r}769 \\
1\end{array}$ & $\begin{array}{r}29315 \\
65\end{array}$ \\
\hline
\end{tabular}




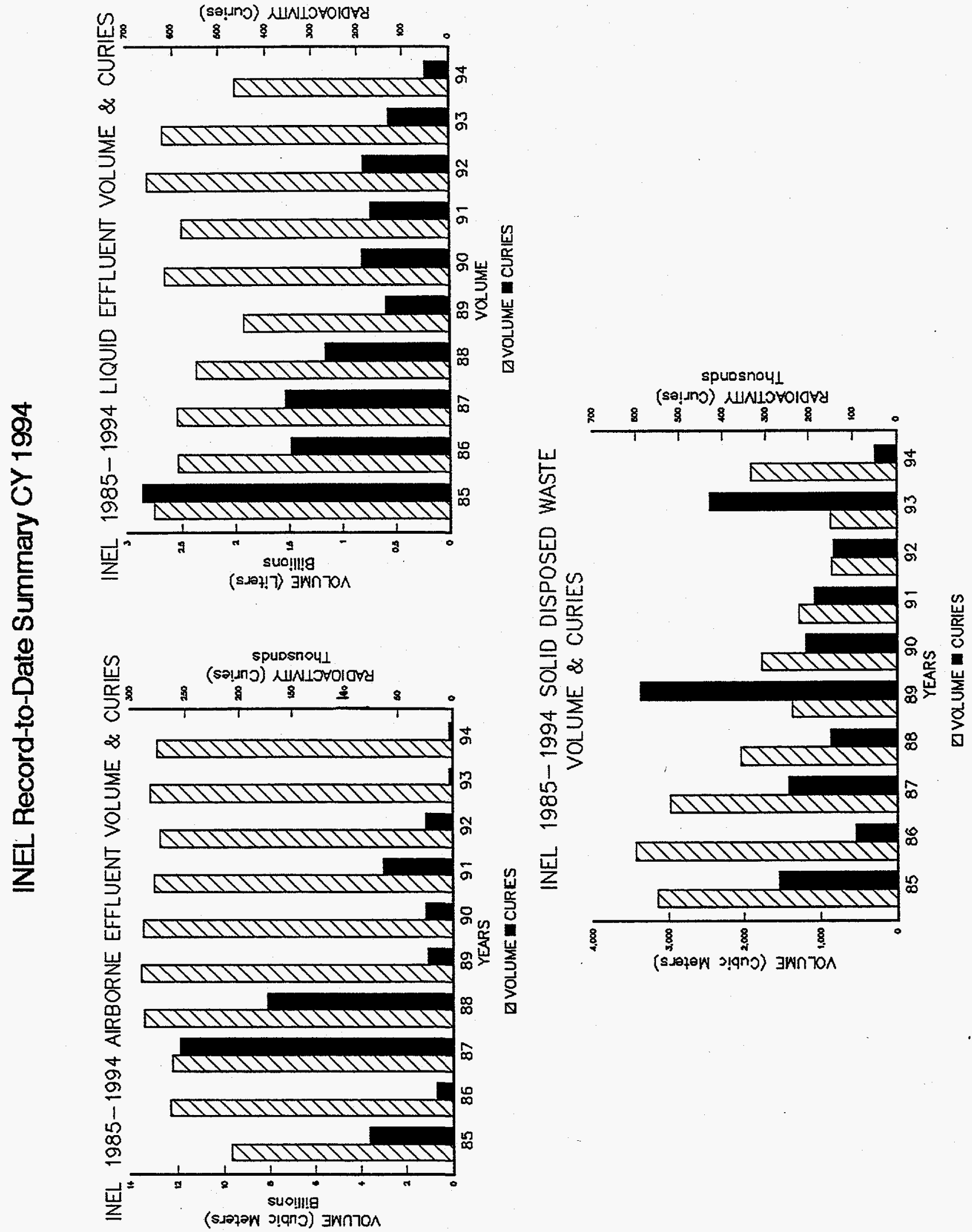




\section{INEL Record-to-Date Summary CY 1994}
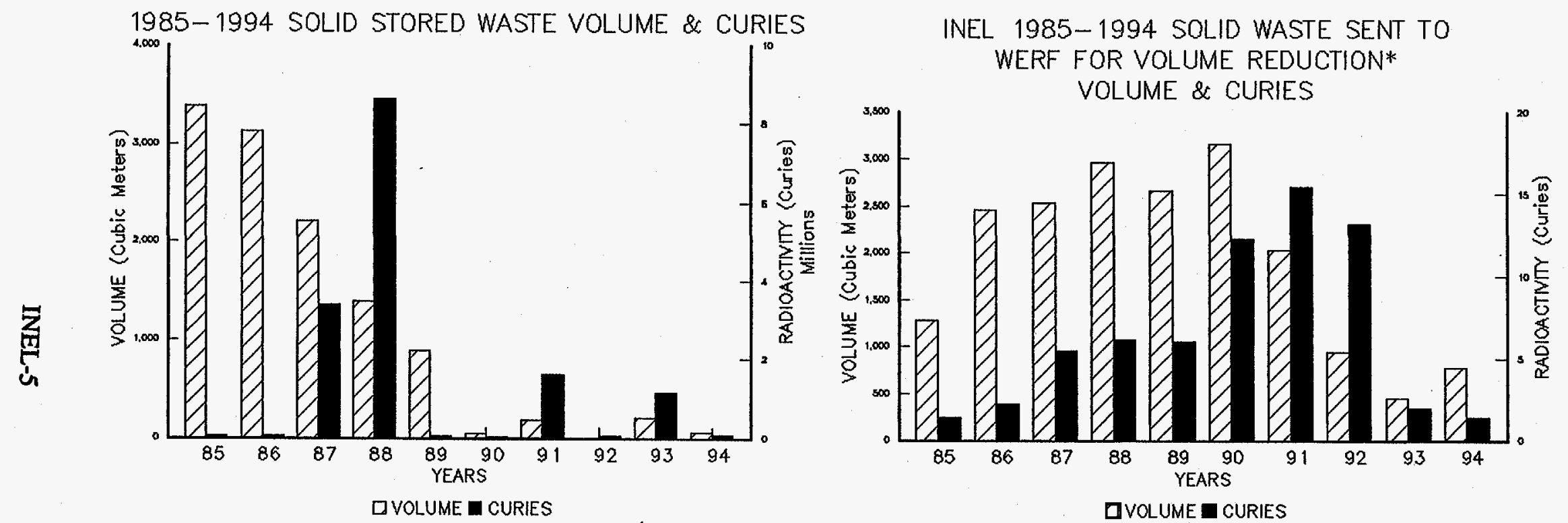

DVOLUME I CURIES

TRA/CPP INCINERABLE WASTE SENT TO

QVOLUME I CURIES

PRIVATE INDUSTRY FOR REDUCTION*

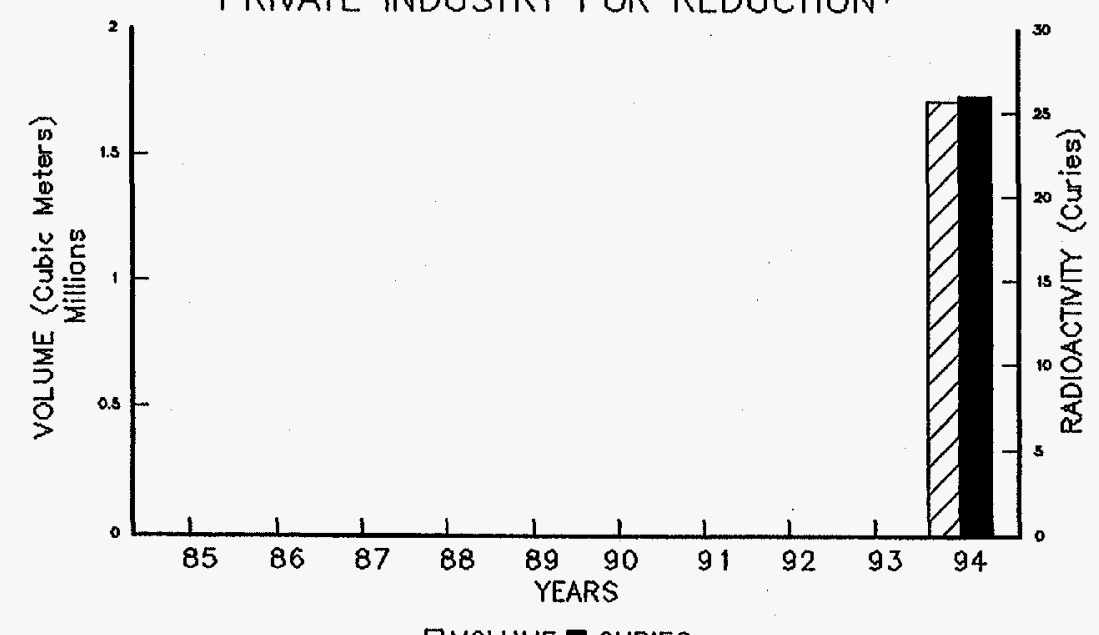

DV VOLUME D CURIES 
INEL Record-to-Date Discharges and Solid Waste

INEL Radioactive Airborne Waste Discharges, Record-to-Date . . . . . . . . . INEL-9

INEL Radioactive Airborne Waste Discharges Record-to-Date Bar Graphs . INEL-11

INEL Radioactive Liquid Waste Discharges, Record-To-Date . . . . . . . . . INEL-15

INEL Radioactive Liquid Waste Discharge Record-To-Date Bar Graphs . . INEL-17

INEL Solid Record-to-Date Summary, Disposed Waste . . . . . . . . . . INEL-20

INEL Solid Record-to-Date Summary Disposed Waste Bar Graphs . . . . INEL-23

INEL Solid Record-to-Date Summary, Stored Waste $\ldots \ldots \ldots \ldots \ldots \ldots \ldots$ INEL-28

INEL Solid Record-to-Date Summary Stored Waste Bar Graphs . . . . . INEL-29

INEL Solid Record-to-Date Summary, ANL Storage Waste and Bar Graph . . . . INEL-30

INEL Solid Record-to-Date Summary, ILTSF Waste . . . . . . . . . . INEL-31

INEL Solid Record-to-Date Summary ILTSF Waste Bar Graphs . . . . . . . INEL-32

INEL Solid Record-to-Date Summary, TSA Waste . . . . . . . . . . . INEL-33

INEL Solid Record-to-Date Summary TSA Waste Bar Graphs . . . . . . . INEL-35

CPP High-Level Liquid/Solid Waste Storage, Record-to-Date . . . . . . . . . . INEL-38

CPP High-Level Liquid/Solid Waste Storage Record-to-Date Bar Graphs . INEL-39

INEL Solid Record-to-Date Summary, WERF Waste $\ldots \ldots \ldots \ldots \ldots \ldots$ INEL-40

INEL Solid Record-to-Date Summary WERF Waste Bar Graphs . . . . . . . INEL-42

INEL Solid Record-to-Date Summary, SCND Waste $\ldots \ldots \ldots \ldots \ldots \ldots$ INEL-46

INEL Solid Record-to-Date SCND Summary CY 1994 Bar Graphs . . . . . . INEL-47 

INEL RADIOACTIVE AIRBORNE WASTE DISCHARGES

RECORD-TO-DATE

\begin{tabular}{|c|c|c|c|c|c|c|c|c|c|c|c|c|c|}
\hline AREA & NEAR & $1952-1984$ & 1985 & 1986 & 1987 & 1988 & 1989 & 1990 & 1991 & 1992 & 1993 & 1994 & TOTAL \\
\hline \multicolumn{14}{|l|}{ ANL } \\
\hline & $\begin{array}{l}\text { VOLLME } \\
\text { CURIES }\end{array}$ & $\begin{array}{l}32147 \\
45525\end{array}$ & $\begin{array}{r}2445 \\
916\end{array}$ & $\begin{array}{r}2406 \\
932\end{array}$ & $\begin{array}{r}2532 \\
889\end{array}$ & $\begin{array}{r}2554 \\
618\end{array}$ & $\begin{array}{r}2540 \\
686\end{array}$ & $\begin{array}{r}2535 \\
716\end{array}$ & $\begin{array}{r}2542 \\
551\end{array}$ & $\begin{array}{r}2542 \\
739\end{array}$ & $\begin{array}{l}2522 \\
1160\end{array}$ & $\begin{array}{l}2520 \\
1154\end{array}$ & $\begin{array}{l}57286 \\
53887\end{array}$ \\
\hline \multicolumn{14}{|l|}{ ARA } \\
\hline & VOLUME & 386 & 47 & 47 & 47 & 32 & & t. & & & & & 560 \\
\hline \multicolumn{14}{|l|}{ CFA } \\
\hline & $\begin{array}{l}\text { VOLLME } \\
\text { CURIES }\end{array}$ & $\begin{array}{r}820 \\
<1\end{array}$ & $\begin{array}{r}140 \\
<1\end{array}$ & $\begin{array}{r}117 \\
<1\end{array}$ & $\begin{array}{l}77 \\
<1\end{array}$ & $\begin{array}{l}59 \\
<1\end{array}$ & $\begin{array}{l}58 \\
<1\end{array}$ & $\begin{array}{l}58 \\
<1\end{array}$ & $\begin{array}{l}58 \\
<1\end{array}$ & $\begin{array}{l}58 \\
<1\end{array}$ & $\begin{array}{l}34 \\
<1\end{array}$ & & $\begin{array}{r}1481 \\
<1\end{array}$ \\
\hline \multicolumn{14}{|l|}{ CPP } \\
\hline & $\begin{array}{l}\text { VOLUME } \\
\text { CURIES }\end{array}$ & $\begin{array}{r}40739 \\
7524886\end{array}$ & $\begin{array}{r}3332 \\
64279\end{array}$ & $\begin{array}{r}3923 \\
10845\end{array}$ & $\begin{array}{r}3911 \\
250918\end{array}$ & $\begin{array}{r}3893 \\
170708\end{array}$ & $\begin{array}{r}3794 \\
20002\end{array}$ & $\begin{array}{r}3827 \\
20002\end{array}$ & $\begin{array}{r}3916 \\
60043\end{array}$ & $\begin{array}{r}3895 \\
20000\end{array}$ & $\begin{array}{r}4244 \\
62\end{array}$ & $\begin{array}{r}4156 \\
<1\end{array}$ & $\begin{array}{r}79632 \\
8141745\end{array}$ \\
\hline \multicolumn{14}{|l|}{ CTF } \\
\hline & $\begin{array}{l}\text { VOLUME } \\
\text { CURIES }\end{array}$ & & & $\begin{array}{r}155 \\
<1\end{array}$ & 63 & & & & & & & & $\begin{array}{r}218 \\
<1\end{array}$ \\
\hline \multicolumn{14}{|l|}{ LOF } \\
\hline & $\begin{array}{l}\text { VOLUME } \\
\text { CURIES }\end{array}$ & $\begin{array}{r}657 \\
31\end{array}$ & $\begin{array}{r}165 \\
8780\end{array}$ & & & & & & & & & & $\begin{array}{r}822 \\
8810\end{array}$ \\
\hline \multicolumn{14}{|l|}{ NRF } \\
\hline & VOLLME & 27521 & 2187 & 2722 & 1630 & 1745 & 2377 & 2151 & 1795 & 1654 & 1646 & 1329 & 46757 \\
\hline & CURIES & 35 & $<1$ & $<1$ & $<1$ & $<1$ & $<1$ & $<1$ & $<1$ & $<1$ & 1 & 2 & 41 \\
\hline \multicolumn{14}{|l|}{ PBF } \\
\hline & VOLLME & 905 & 62 & 57 & 58 & 60 & 60 & 62 & 63 & 58 & 52 & 59 & 1495 \\
\hline & CURIES & $<1$ & $<1$ & $<1$ & $<1$ & $<1$ & $<1$ & $<1$ & $<1$ & $<1$ & $<1$ & $<1$ & $<1$ \\
\hline \multicolumn{14}{|l|}{ PER } \\
\hline & VOLUME & $* *$ & & & & & & & & & & & $* *$ \\
\hline & CURIES & 6 & & & & & & & & & & & 6 \\
\hline \multicolumn{14}{|l|}{ SMC } \\
\hline & VOLLME & & 8 & 1424 & 1962 & 3046 & 2812 & 3031 & 3.122 & 3047 & 3141 & 3166 & 24758 \\
\hline & CURIES & & $<1$ & $<1$ & $<1$ & $<1$ & $<1$ & $<1$ & $<1$ & $<1$ & $<1$ & $<1$ & $<1$ \\
\hline \multicolumn{14}{|l|}{ TAN } \\
\hline & $\begin{array}{l}\text { VOLUME } \\
\text { CURTES }\end{array}$ & $\begin{array}{l}14983 \\
53725\end{array}$ & 173 & 332 & 660 & 645 & 632 & 492 & 191 & 182 & 191 & 197 & 18677 \\
\hline \multicolumn{14}{|l|}{ TRA } \\
\hline & $\begin{array}{l}\text { VOLUME } \\
\text { CURIES }\end{array}$ & $\begin{array}{r}22506 \\
5425861\end{array}$ & $\begin{array}{l}1076 \\
3288\end{array}$ & $\begin{array}{l}1089 \\
2741\end{array}$ & $\begin{array}{l}1199 \\
3140\end{array}$ & $\begin{array}{l}1190 \\
2609\end{array}$ & $\begin{array}{l}1069 \\
1682\end{array}$ & $\begin{array}{l}1130 \\
3741\end{array}$ & $\begin{array}{l}1140 \\
3284\end{array}$ & $\begin{array}{l}1130 \\
3439\end{array}$ & $\begin{array}{l}1155 \\
1495\end{array}$ & $\begin{array}{l}1190 \\
1074\end{array}$ & $\begin{array}{r}33873 \\
5452352\end{array}$ \\
\hline
\end{tabular}


RECORD-TO-DATE

\begin{tabular}{|c|c|c|c|c|c|c|c|c|c|c|c|c|}
\hline AREA/YEAR & $1952-1984$ & 1985 & 1986 & 1987 & 1988 & 1989 & 1990 & 1991 & 1992 & 1993 & 1994 & TOTAL \\
\hline \multicolumn{13}{|l|}{ WER } \\
\hline VOLUME & & & 16 & 72 & 161 & 156 & 164 & 137 & 135 & 155 & 239 & 1235 \\
\hline CURIES & & & $<1$ & $<1$ & $<1$ & $<1$ & $<1$ & $<1$ & $<1$ & & $<1$ & $<1$ \\
\hline \multicolumn{13}{|l|}{ WMC } \\
\hline VOLUME & & & & 9 & 4 & 15 & & & 14 & 10 & & 52 \\
\hline CURIES & & & & $<1$ & $<1$ & $<1$ & & & $<1$ & $<1$ & & $<1$ \\
\hline \multicolumn{13}{|l|}{ TOTAL } \\
\hline $\begin{array}{l}\text { VOLUME } \\
\text { CURIES }\end{array}$ & $\begin{array}{r}140664 \\
13050065\end{array}$ & $\begin{array}{r}9636 \\
77262\end{array}$ & $\begin{array}{l}12289 \\
14518\end{array}$ & $\begin{array}{r}12220 \\
254948\end{array}$ & $\begin{array}{r}13388 \\
173935\end{array}$ & $\begin{array}{l}135114 \\
22370\end{array}$ & $\begin{array}{l}13450 \\
24459\end{array}$ & $\begin{array}{l}12963 \\
63879\end{array}$ & $\begin{array}{l}12715 \\
24179\end{array}$ & $\begin{array}{r}13150 \\
2719\end{array}$ & $\begin{array}{r}12856 \\
2230\end{array}$ & $\begin{array}{r}266845 \\
13710563\end{array}$ \\
\hline
\end{tabular}

VOLUME IN MILLIONS OF CUBIC METERS

DETAILS MAY NOT ADD UP TO TOTALS BECAUSE OF ROUNDING 

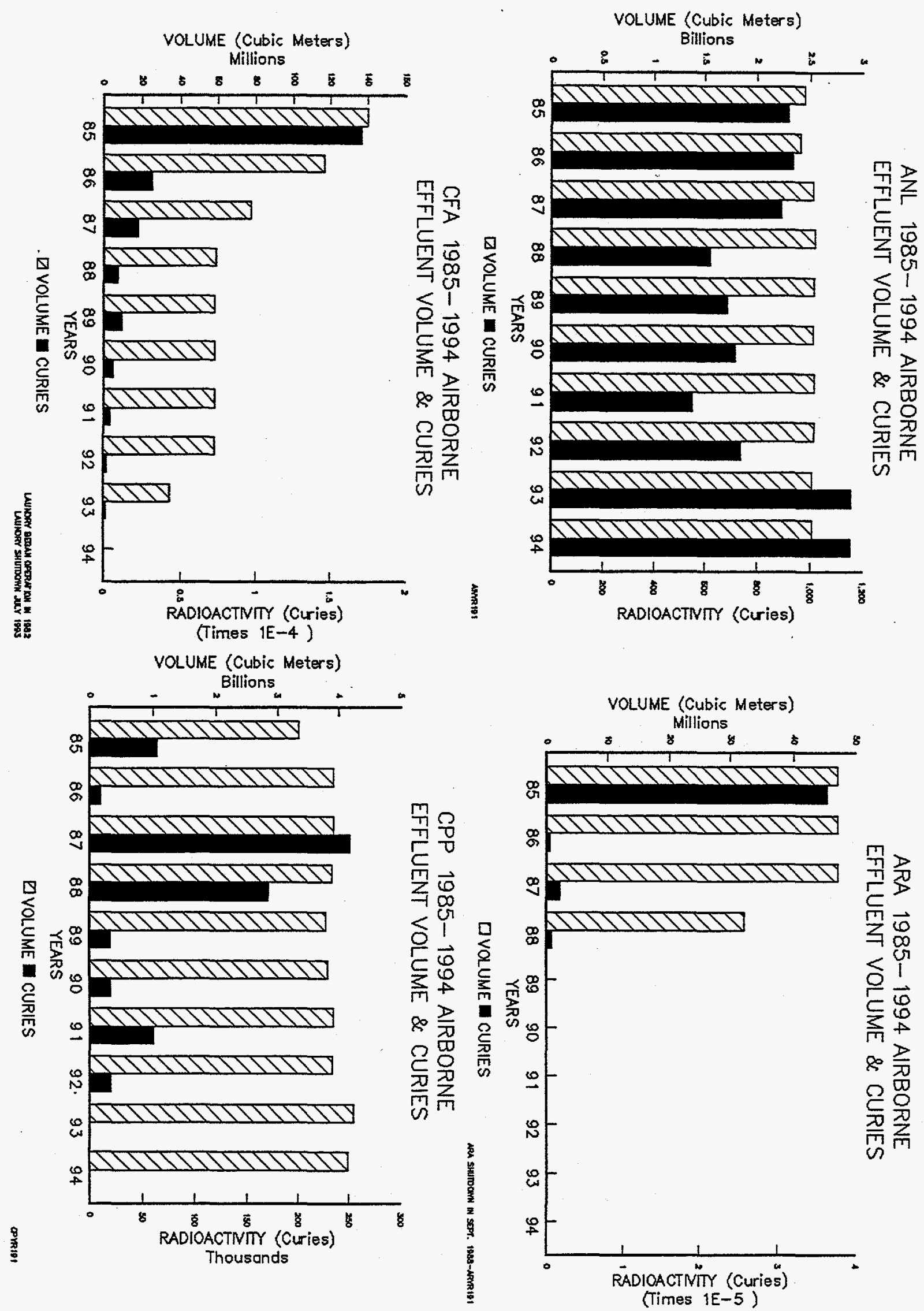
INEL Radioactive Airborne Waste Discharges Record-to-Date

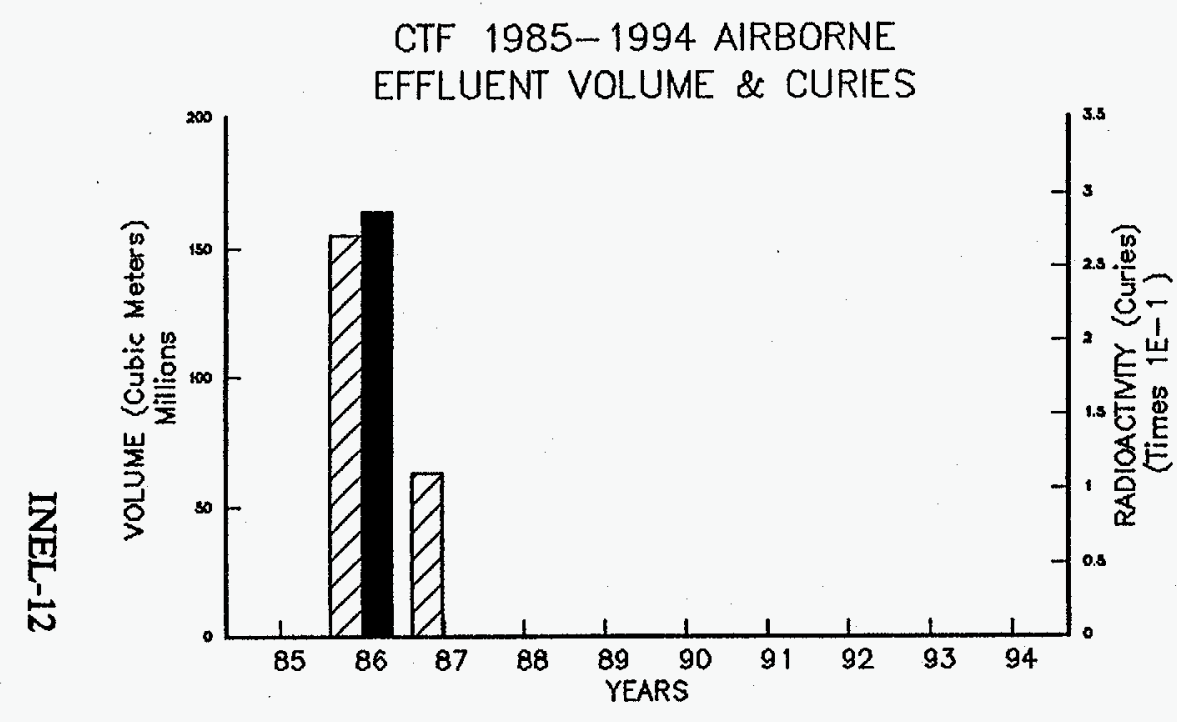

IV VOLUME I CURIES

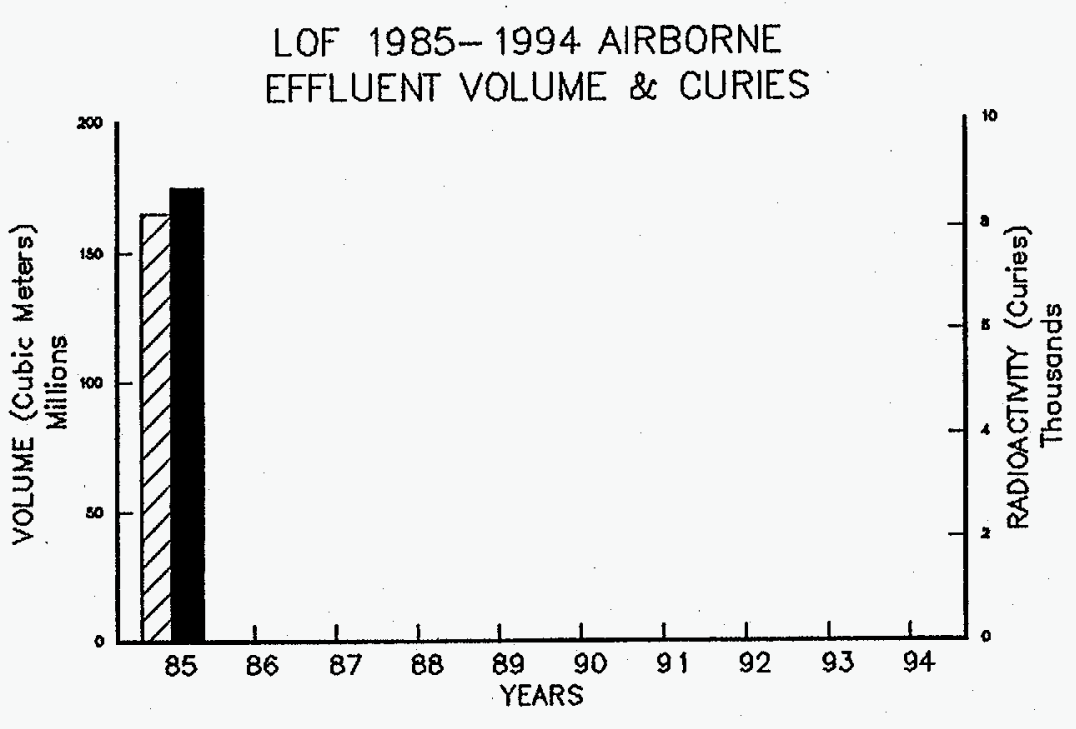

DVOLUME $\mathbf{D}$ CURIES

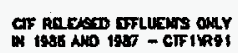

NRF 1985- 1994 AIRBORNE

EFFLUENT VOLUME \& CURIES

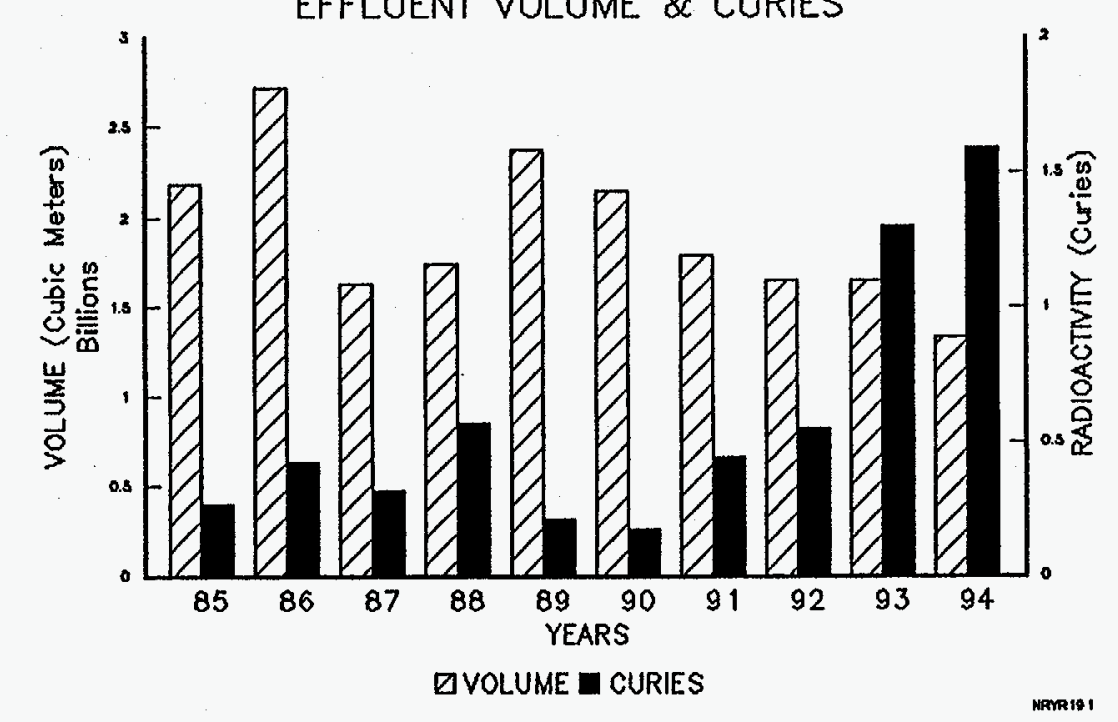




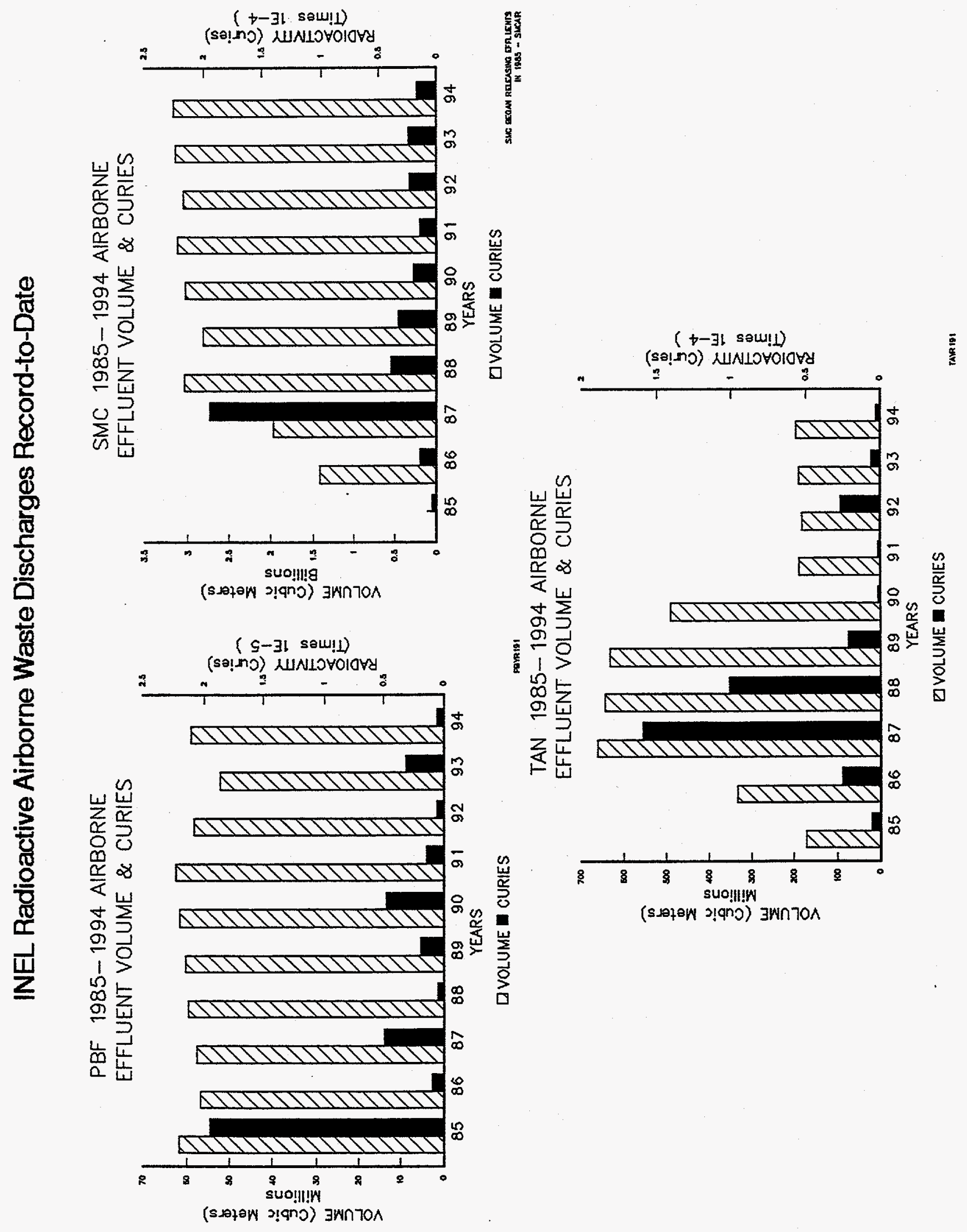




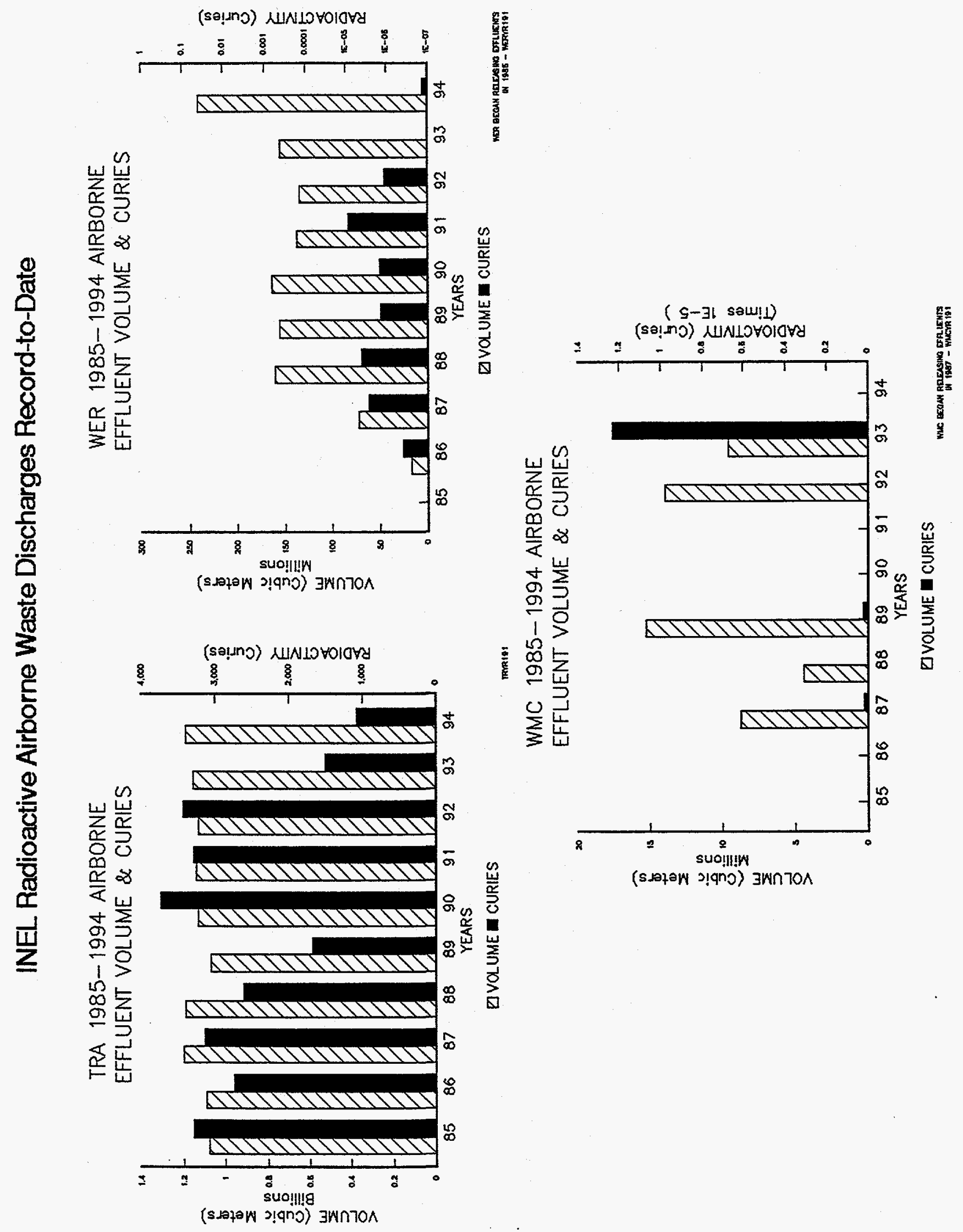


RECORD-TO-DATE

\begin{tabular}{|c|c|c|c|c|c|c|c|c|c|c|c|c|c|}
\hline AREA & NEAR & $1952-1984$ & 1985 & 1986 & 1987 & 1988 & 1989 & 1990 & 1991 & 1992 & 1993 & 1994 & TOTAL \\
\hline \multicolumn{14}{|l|}{ ANL } \\
\hline & VOLUME & 66 & 2 & 2 & 2 & 2 & 1 & 2 & 2 & 1 & 5 & 5 & 91 \\
\hline & CURIES & 21 & $<1$ & $<1$ & $<1$ & $<1$ & $<\overline{1}$ & $<1$ & $<1$ & $<1$ & $<1$ & $<1$ & 23 \\
\hline \multicolumn{14}{|l|}{ ARA } \\
\hline & VOLUME & 61 & & $<1$ & 2 & 1 & & & & & & & 65 \\
\hline & CURIES & $<1$ & & $<1$ & $<1$ & $<1$ & & & & & & & $<1$ \\
\hline \multicolumn{14}{|l|}{ CFA } \\
\hline & VOLUME & 4579 & 166 & 104 & 89 & 62 & 117 & 154 & 192 & 160 & 44 & & 5668 \\
\hline & CURIES & 44 & 4 & 3 & 2 & 1 & 2 & 2 & 3 & 3 & $<i$ & & 65 \\
\hline \multicolumn{14}{|l|}{ CPp } \\
\hline & VOLUME & 42644 & 2020 & 2183 & 2268 & 2140 & 1664 & 2357 & 2107 & 2433 & 2515 & 1964 & 64295 \\
\hline & CURIES & 21298 & 393 & 251 & 216 & 89 & $<1$ & $<1$ & 2 & $<1$ & $<1$ & $<1$ & 22250 \\
\hline \multicolumn{14}{|l|}{ CTF } \\
\hline & $\begin{array}{l}\text { VOLUME } \\
\text { CURIES }\end{array}$ & & & $\begin{array}{l}71 \\
<1\end{array}$ & 22 & & & & & & & & $\begin{array}{l}93 \\
<1\end{array}$ \\
\hline \multicolumn{14}{|l|}{ LOF } \\
\hline & VOLUME & 2386 & 427 & & & & & & & & & & 2813 \\
\hline & CURIES & $<1$ & $<1$ & & & & & & & & & & $<1$ \\
\hline \multicolumn{14}{|l|}{ NRF } \\
\hline & VOLUME & 1569 & & & & & & & & $<1$ & & & 1569 \\
\hline & CURIES & 349 & & & & & & & & $<1$ & & & 349 \\
\hline \multicolumn{14}{|l|}{ PBF } \\
\hline & VOLUME & 9 & & & & & & & & & & & 9 \\
\hline & CURIES & $<1$ & & & & & & & & & & & $<1$ \\
\hline \multicolumn{14}{|l|}{ PER } \\
\hline & VOLUME & 76 & & & & & & & & & & & 76 \\
\hline & CURIES & 155 & & & & & & & & & & & 155 \\
\hline \multicolumn{14}{|l|}{ TAN } \\
\hline & VOLUME & 2070 & 51 & 73 & 82 & 81 & 67 & 58 & 90 & 116 & 24 & & 2712 \\
\hline & CURIES & 58 & $<1$ & $<1$ & $<1$ & $<1$ & $<1$ & CI & $<1$ & $<1$ & 2 & & 61 \\
\hline \multicolumn{14}{|l|}{ TRA } \\
\hline & $\begin{array}{l}\text { VOLLME } \\
\text { CURTES }\end{array}$ & $\begin{array}{l}18785 \\
52011\end{array}$ & 78 & 94 & 70 & 70 & 73 & 71 & 99 & 88 & 71 & 25 & 19523 \\
\hline \multirow{2}{*}{\multicolumn{14}{|c|}{$\operatorname{manc}$}} \\
\hline & & & & & & & & & & & & & \\
\hline & $\begin{array}{l}\text { VOLLME } \\
\text { CURIES }\end{array}$ & $<1$ & & & & & & & & & & & $<1$ \\
\hline
\end{tabular}


RECORD-TO-DATE

\begin{tabular}{|c|c|c|c|c|c|c|c|c|c|c|c|c|}
\hline AREA/YEAR & $1952-1984$ & 1985 & 1986 & 1987 & 1988 & 1989 & 1990 & 1991 & 1992 & 1993 & 1994 & TOTAL \\
\hline \multicolumn{13}{|l|}{ TOTAL } \\
\hline $\begin{array}{l}\text { VOLUME } \\
\text { CURIES }\end{array}$ & $\begin{array}{l}72243 \\
73936\end{array}$ & $\begin{array}{r}2744 \\
667\end{array}$ & $\begin{array}{r}2529 \\
346\end{array}$ & $\begin{array}{r}2536 \\
357\end{array}$ & $\begin{array}{r}2357 \\
270\end{array}$ & $\begin{array}{r}1922 \\
137\end{array}$ & $\begin{array}{r}2642 \\
189\end{array}$ & $\begin{array}{r}2490 \\
170\end{array}$ & $\begin{array}{r}2799 \\
187\end{array}$ & $\begin{array}{r}2659 \\
130\end{array}$ & $\begin{array}{r}1995 \\
50\end{array}$ & $\begin{array}{l}96915 \\
76439\end{array}$ \\
\hline
\end{tabular}

* VOLUME IN MILLION LITERS

* FFOR THE YEARS 1962-70 THE SHORT-LIVED RADIOACTIVITY (LESS THAN 2-3 DAY HALF LIFE) WERE NOT REPORTED

DETAILS MAY NOT ADD UP TO TOTALS BECAUSE OF ROUNDING 
INEL Radioactive Liquid Waste Discharge Record-to-Date
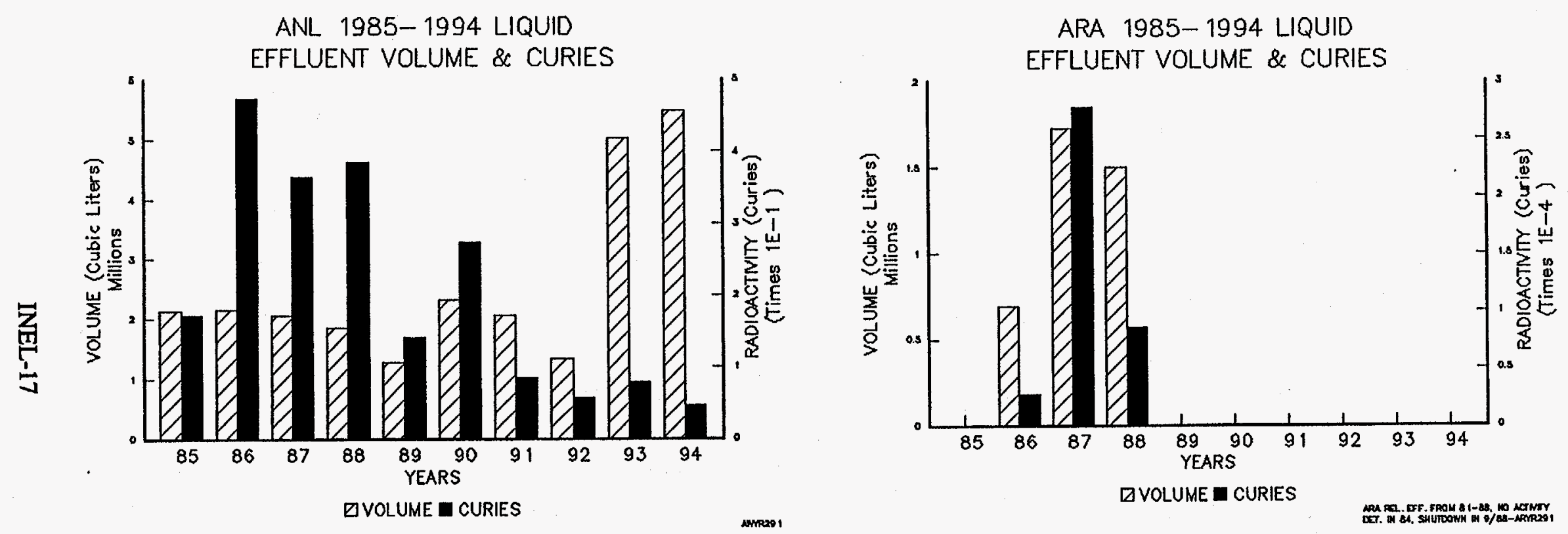

CFA 1985-1994 LIQUID

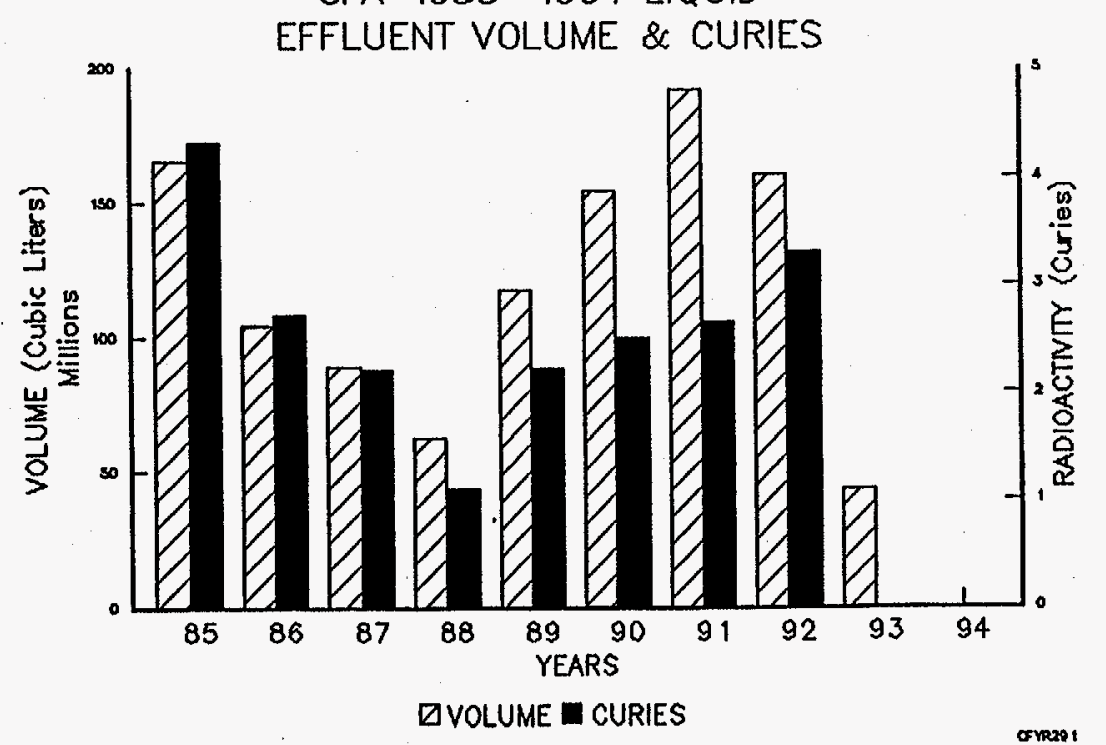




\section{INEL Radioactive Liquid Waste Discharge Record-to-Date}

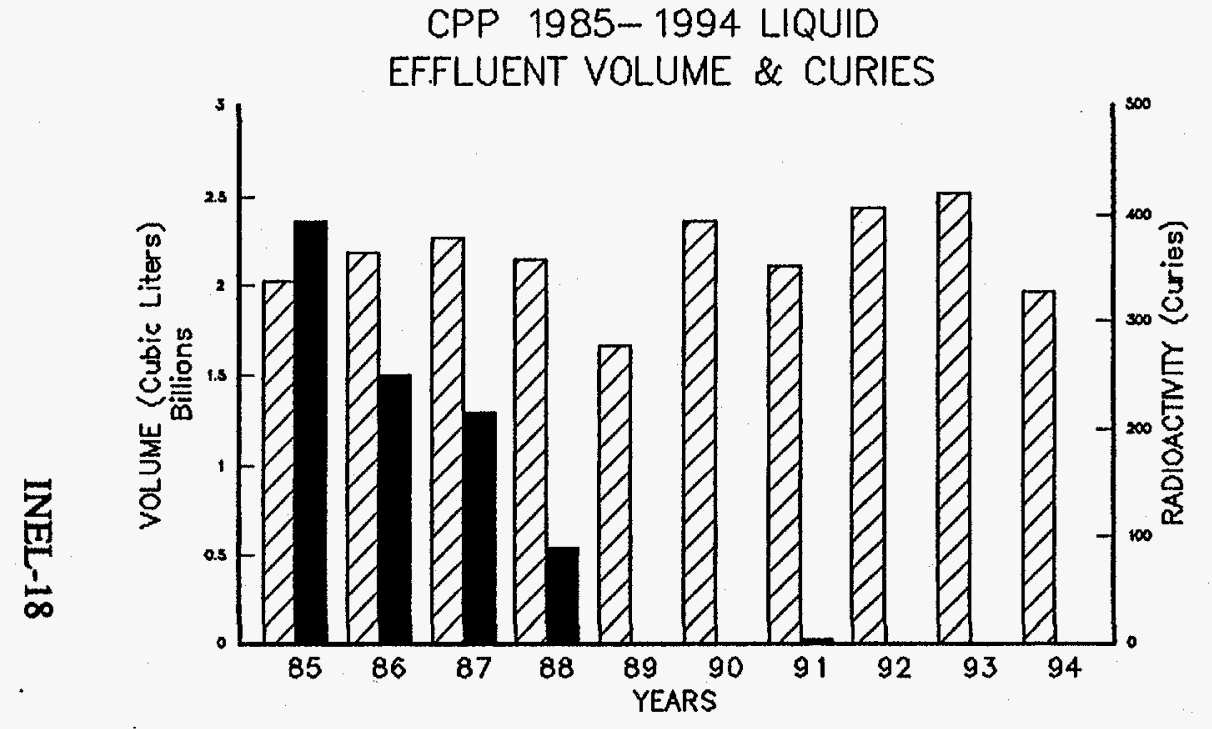

D YOLUME I CURIES

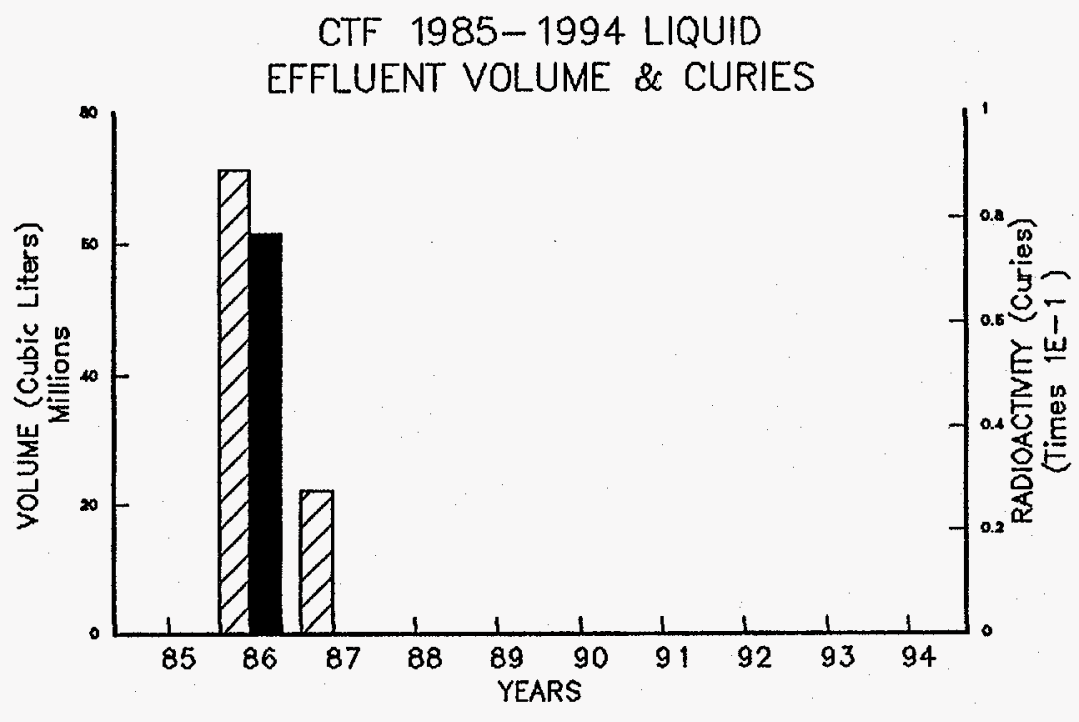

DVOLUME CURIES

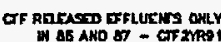

LOF 1985- 1994 LIQUID

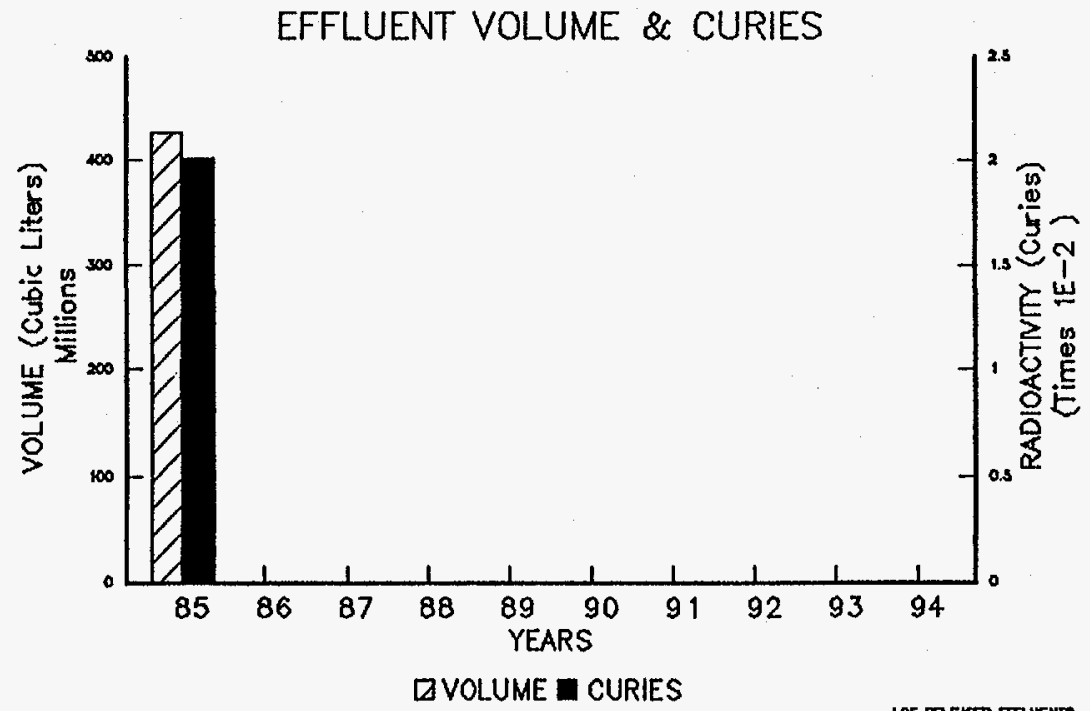




\section{INEL Radioactive Liquid Waste Discharge Record-to-Date}
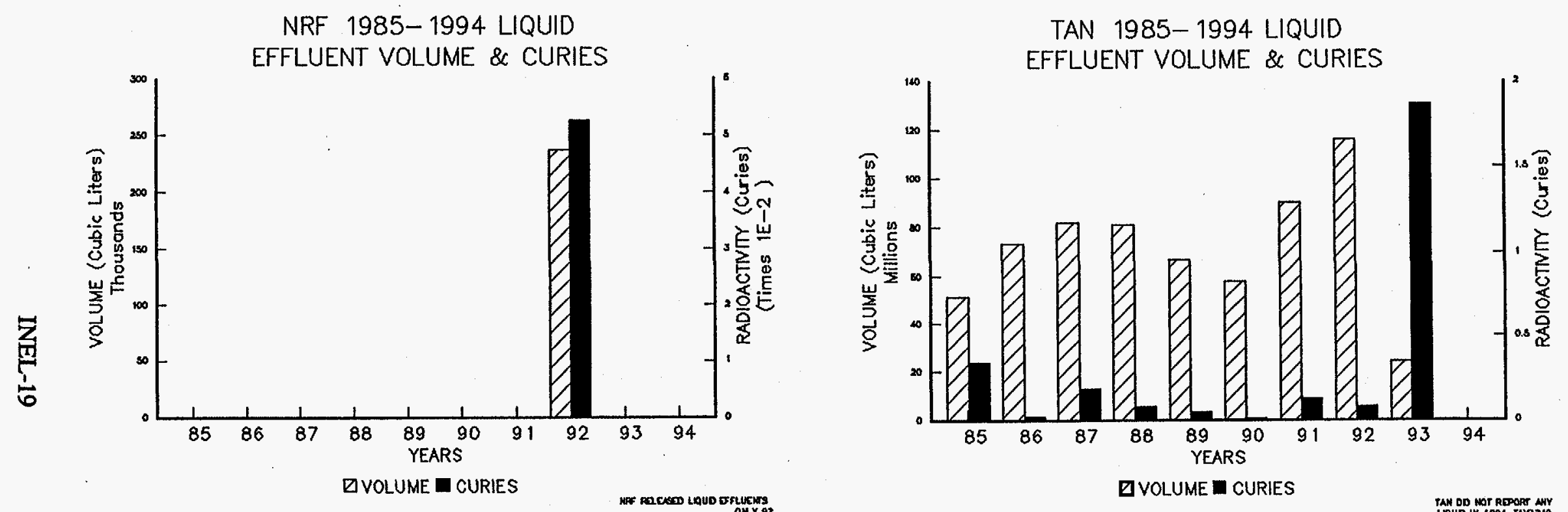

TRA 1985-1994 LIQUID

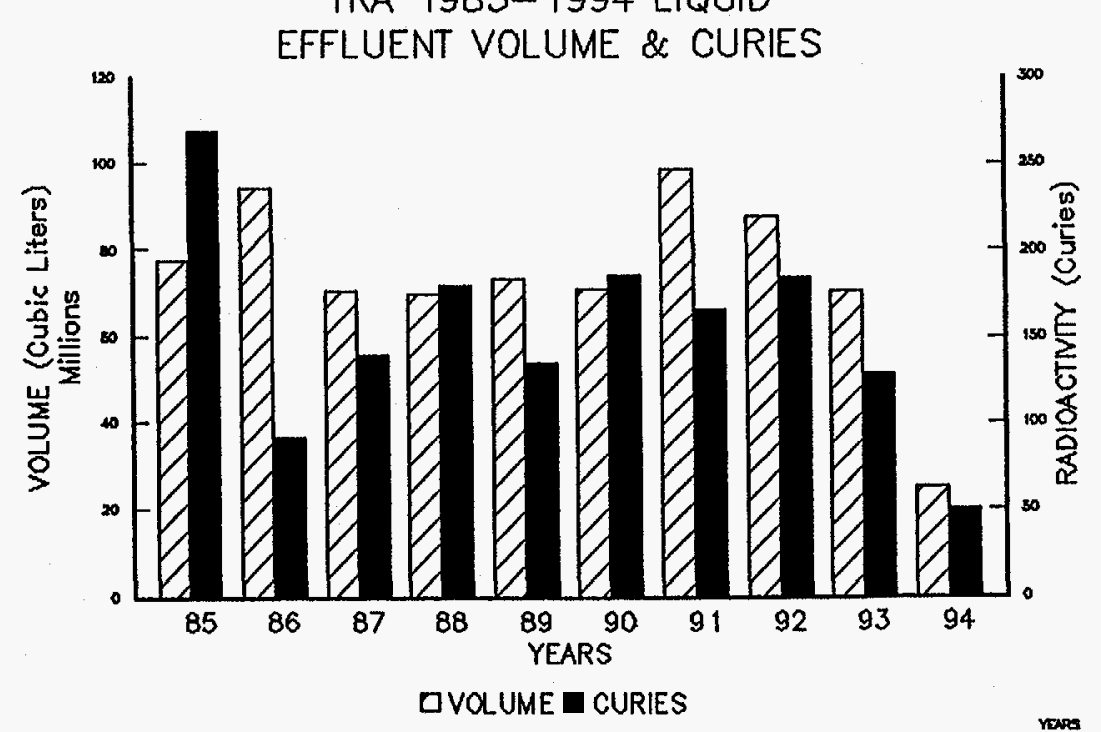


OISPOSED WASTE

\begin{tabular}{|c|c|c|c|c|c|c|c|c|c|c|c|c|c|}
\hline AREA & NEAR & $1952-1984$ & 1985 & 1986 & 1987 & 1988 & 1989 & 1990 & 1991 & 1992 & 1993 & 1994 & TOTAL. \\
\hline \multicolumn{14}{|l|}{ ALE } \\
\hline & $\begin{array}{l}\text { VOLUME } \\
\text { CURIES }\end{array}$ & $\begin{array}{r}3986 \\
510\end{array}$ & $\begin{array}{r}592 \\
71\end{array}$ & $\begin{array}{r}306 \\
10\end{array}$ & $\begin{array}{l}309 \\
840\end{array}$ & $\begin{array}{r}204 \\
50\end{array}$ & & & & & & & $\begin{array}{l}5398 \\
1481\end{array}$ \\
\hline \multicolumn{14}{|l|}{ ANL } \\
\hline & $\begin{array}{l}\text { VOLUME } \\
\text { CURIES }\end{array}$ & $\begin{array}{r}7265 \\
1231127\end{array}$ & $\begin{array}{r}121 \\
126651\end{array}$ & $\begin{array}{r}135 \\
57382\end{array}$ & $\begin{array}{r}109 \\
204682\end{array}$ & $\begin{array}{r}473 \\
142614\end{array}$ & $\begin{array}{r}172 \\
463320\end{array}$ & $\begin{array}{r}65 \\
130511\end{array}$ & $\begin{array}{r}30 \\
82261\end{array}$ & $\begin{array}{r}56 \\
92896\end{array}$ & $\begin{array}{r}77 \\
87058\end{array}$ & $\begin{array}{r}211 \\
12301\end{array}$ & $\begin{array}{r}8713 \\
2630803\end{array}$ \\
\hline \multicolumn{14}{|l|}{ ARA } \\
\hline & $\begin{array}{l}\text { VOLUME } \\
\text { CURIES }\end{array}$ & $\begin{array}{r}396 \\
4019\end{array}$ & $\begin{array}{r}17 \\
5\end{array}$ & $<1$ & $\begin{array}{l}20 \\
<1\end{array}$ & & & & & & & $\begin{array}{r}101 \\
<1\end{array}$ & $\begin{array}{r}536 \\
4025\end{array}$ \\
\hline \multicolumn{14}{|l|}{ BEN } \\
\hline & VOLUME & & & $<1$ & & & & & & & & & $<1$ \\
\hline & CURIES & & & $<1$ & & & & & & & & & $<1$ \\
\hline \multicolumn{14}{|l|}{ BNL } \\
\hline & VOLUME & 5 & & & & & & & & & & & 5 \\
\hline & CURIES & $<1$ & & & & & & & & & & & $<1$ \\
\hline \multicolumn{14}{|l|}{ CEG } \\
\hline & $\begin{array}{l}\text { VOLUME } \\
\text { CURIES }\end{array}$ & & & & & & & & & & $\begin{array}{r}22 \\
1967\end{array}$ & . & $\begin{array}{r}22 \\
1967\end{array}$ \\
\hline \multicolumn{14}{|l|}{ CFA } \\
\hline & VOLUME & 2467 & 9 & 16 & 4 & 5 & 4 & 45 & & & 84 & 42 & 2675 \\
\hline & CURIES & 382 & $<1$ & $<1$ & 4 & $<1$ & $<1$ & $<1$ & & & $<1$ & $<1$ & 385 \\
\hline \multicolumn{14}{|l|}{ CPP } \\
\hline & VOLUME & 27757 & 807 & 948 & 1151 & 578 & 513 & 752 & 544 & 129 & 7 & 127 & 33314 \\
\hline & CURIES & 613701 & 112 & 214 & 218 & 192 & 115 & 211 & 24 & 2 & $<1$ & 2 & 614791 \\
\hline \multirow[t]{3}{*}{ CTF } & & & & i & & & & & & & & & \\
\hline & VOLLAME & & & 805 & 251 & 27 & & & & & 54 & & 1138 \\
\hline & CURIES & & & 277 & 25 & $<1$ & & & & & $<1$ & & 303 \\
\hline \multicolumn{14}{|l|}{$D+D$} \\
\hline & VOLLME & 2316 & 34 & 53 & 8 & 95 & 24 & 21 & 58 & 185 & 344 & 654 & 3791 \\
\hline & CURIES & 5692 & $<1$ & $<1$ & $<1$ & $<1$ & $<1$ & $<1$ & $<1$ & $<1$ & 2 & $<1$ & 5695 \\
\hline \multicolumn{14}{|l|}{ LOF } \\
\hline & $\begin{array}{l}\text { VOLUME } \\
\text { CURTES }\end{array}$ & 24 & 49 & & & & · & & & & & & $\begin{array}{r}73 \\
3\end{array}$ \\
\hline \multicolumn{14}{|l|}{ NRF } \\
\hline & $\begin{array}{l}\text { VOLUME } \\
\text { CURIES }\end{array}$ & $\begin{array}{r}20194 \\
4015925\end{array}$ & $\begin{array}{r}347 \\
141753\end{array}$ & $\begin{array}{r}270 \\
35930\end{array}$ & $\begin{array}{r}171 \\
29665\end{array}$ & $\begin{array}{r}111 \\
6732\end{array}$ & $\begin{array}{r}134 \\
126486\end{array}$ & $\begin{array}{r}419 \\
74087\end{array}$ & $\begin{array}{r}270 \\
102849\end{array}$ & $\begin{array}{r}159 \\
49795\end{array}$ & $\begin{array}{r}146 \\
42259\end{array}$ & $\begin{array}{r}222 \\
36900\end{array}$ & $\begin{array}{r}22444 \\
4662383\end{array}$ \\
\hline
\end{tabular}


INEL SOLID RECORD-TO-DATE SUMMARY

DISPOSED WASTE

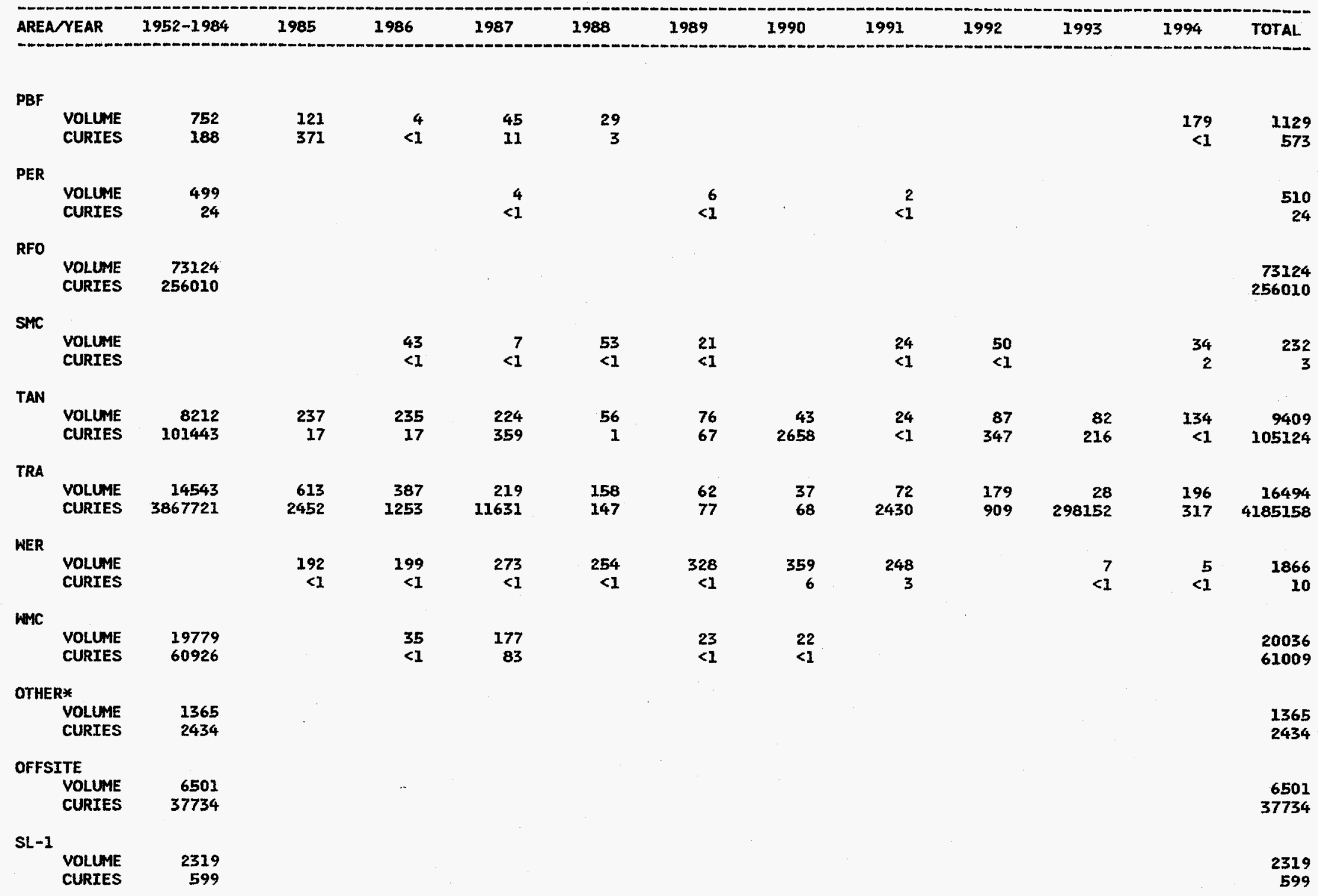




\section{DISPOSED WASTE}

\begin{tabular}{|c|c|c|c|c|c|c|c|c|c|c|c|c|}
\hline AREA/YEAR & $1952-1984$ & 1985 & 1986 & 1987 & 1988 & 1989 & 1990 & 1991 & 1992 & 1993 & 1994 & TOTAL \\
\hline \multicolumn{13}{|l|}{ TOTAL } \\
\hline $\begin{array}{l}\text { VOLUME\# } \\
\text { CURIES" }\end{array}$ & $\begin{array}{r}191504 \\
10198432\end{array}$ & $\begin{array}{r}3140 \\
271433\end{array}$ & $\begin{array}{r}3437 \\
95084\end{array}$ & $\begin{array}{r}2969 \\
247519\end{array}$ & $\begin{array}{r}2045 \\
149740\end{array}$ & $\begin{array}{r}1364 \\
590065\end{array}$ & $\begin{array}{r}1762 \\
207541\end{array}$ & $\begin{array}{r}1272 \\
187566\end{array}$ & $\begin{array}{r}844 \\
143948\end{array}$ & $\begin{array}{r}852 \\
429654\end{array}$ & $\begin{array}{r}1906 \\
49524\end{array}$ & $\begin{array}{r}211095 \\
12570507\end{array}$ \\
\hline
\end{tabular}

VOLUME IN CUBIC METERS

*ML-1, GCRF, OMRE

FFACILITY TOTALS (EXCEPT FOR RFO) DO NOT INCLUDE DATA FOR 1952-1960:

THIS INFORMATION ( 18460 CUBIC METERS AND 60920 CURIES) IS REPORTED IN LUMP SUM UNDER WMC FOR 1960

DETAILS MAY NOT ADD UP TO TOTALS BECAUSE OF ROUNDING 


\section{INEL Solid Record-to-Date Summary Disposed Waste}

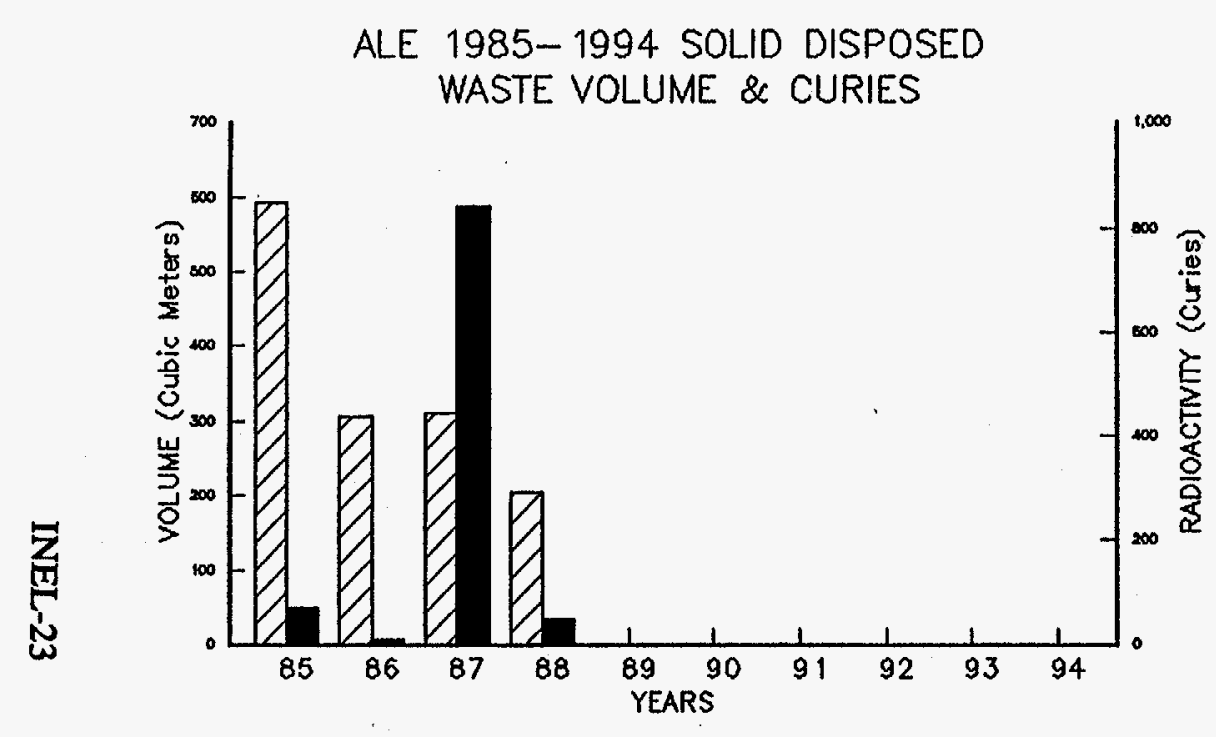

$\square$ VOLUME $\square$ CURIES

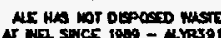

ARA 1985- 1994 SOLID DISPOSED

WASTE VOLUME \& CURIES

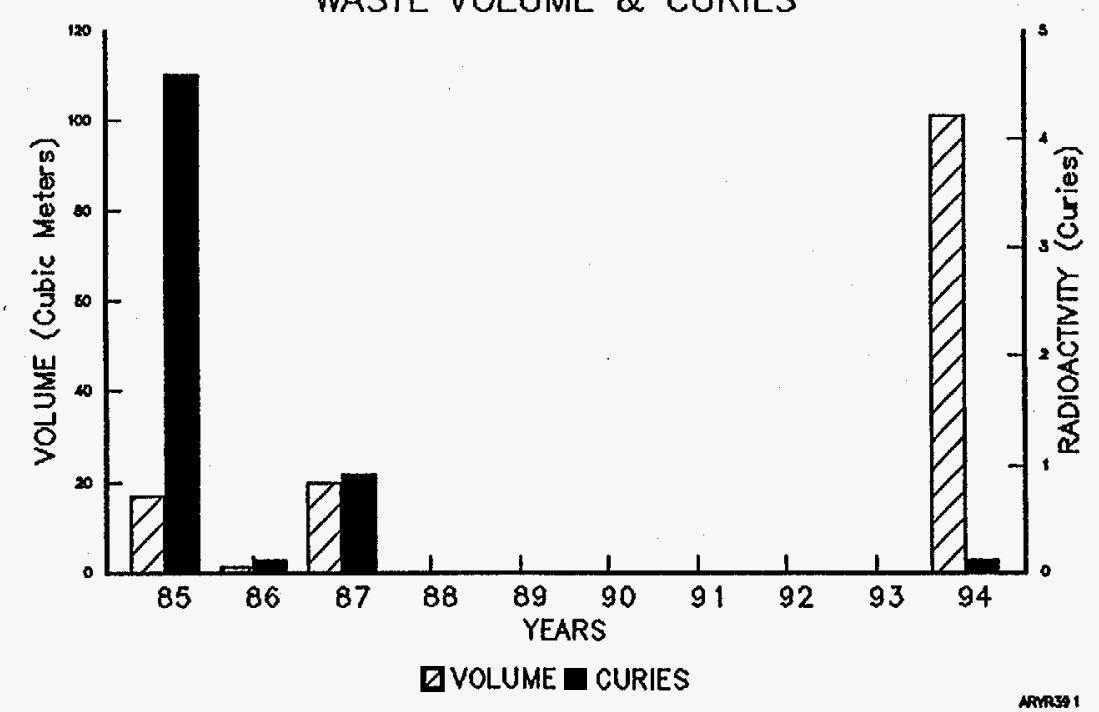

ANL 1985-1994 SOLID DISPOSED WASTE VOLUME \& CURIES

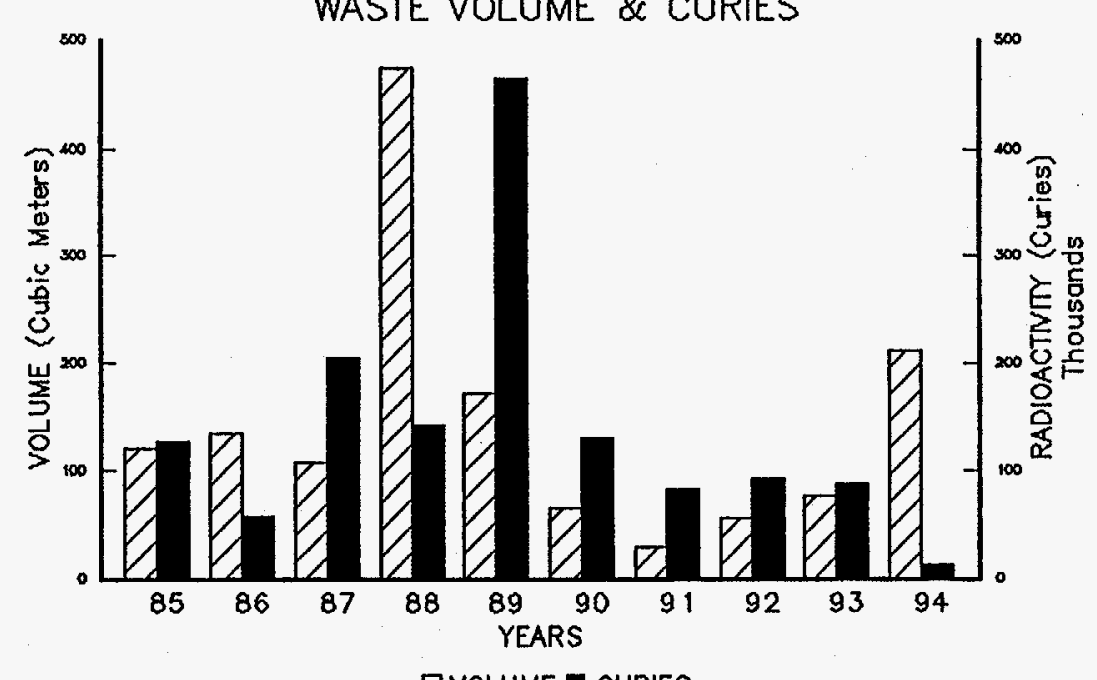

D VOLUME I CURIES

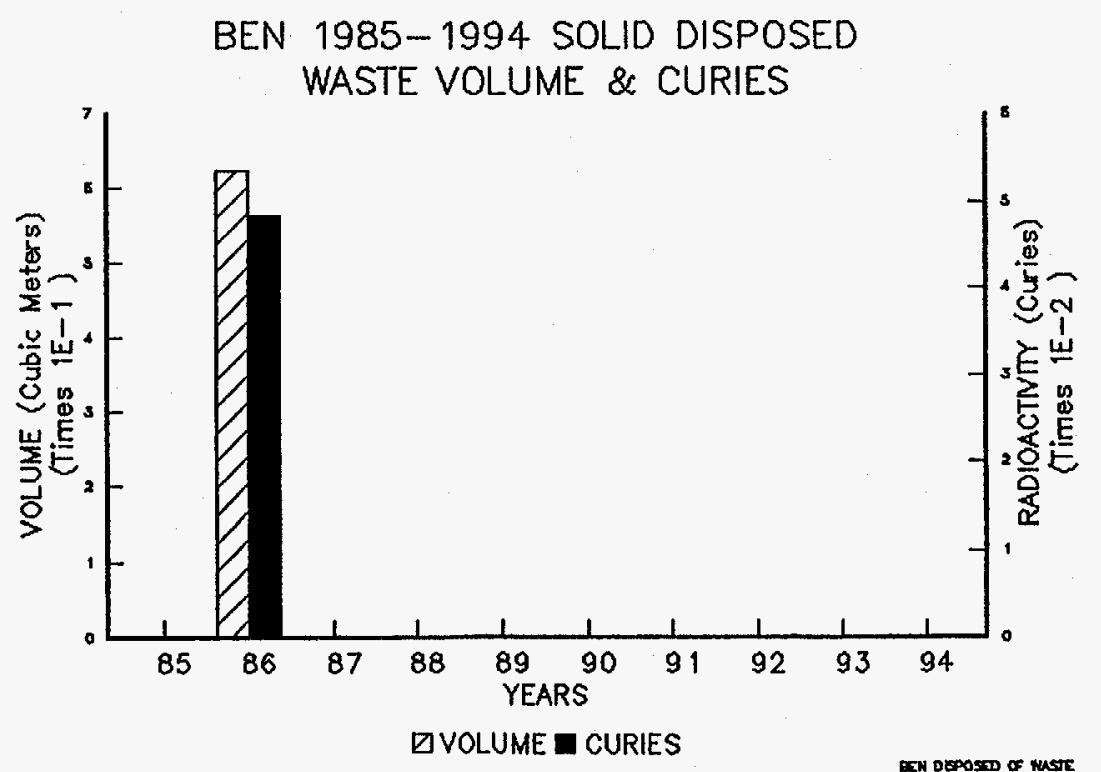


INEL Solid Record-to-Date Summary Disposed Waste
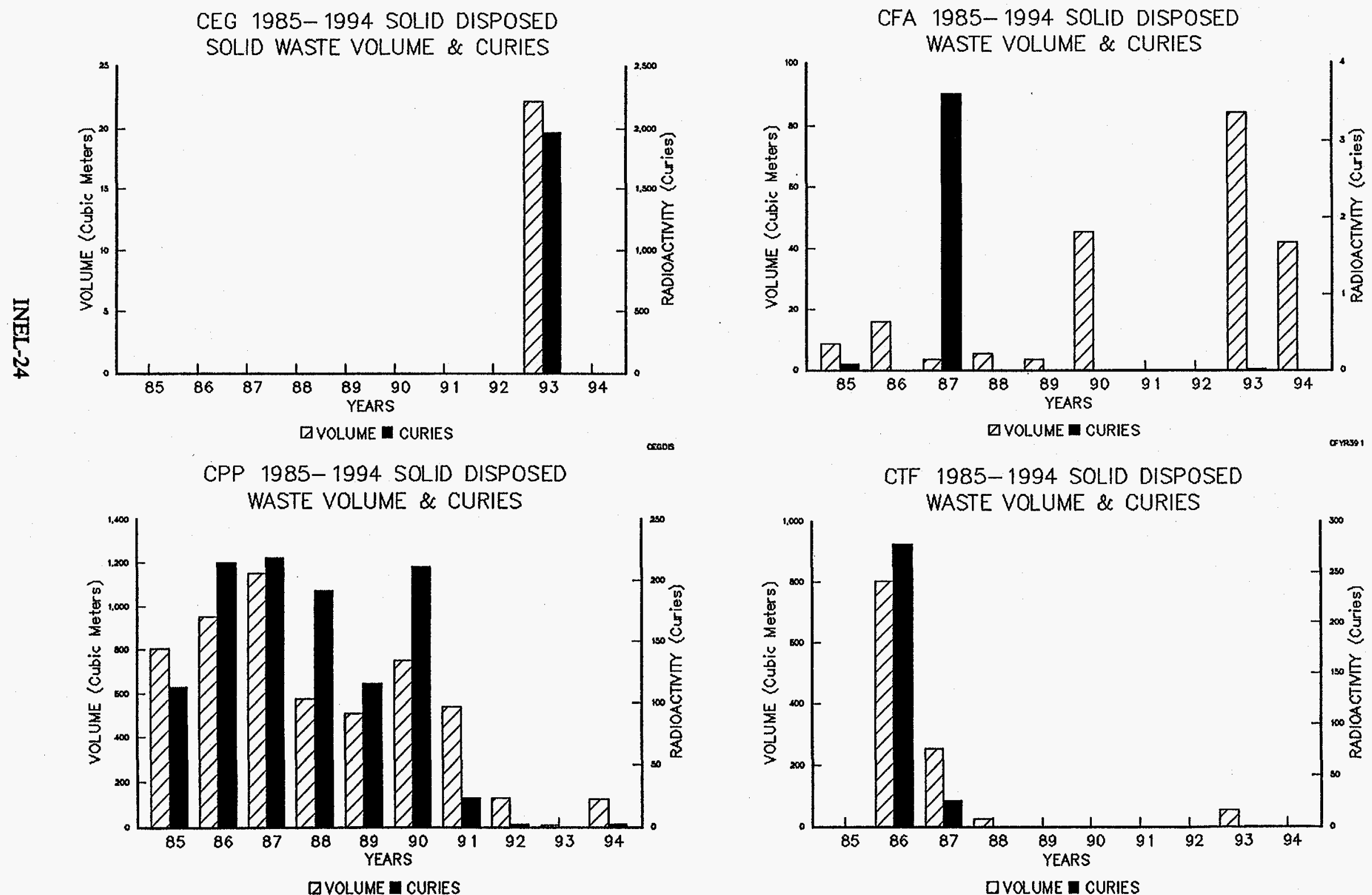

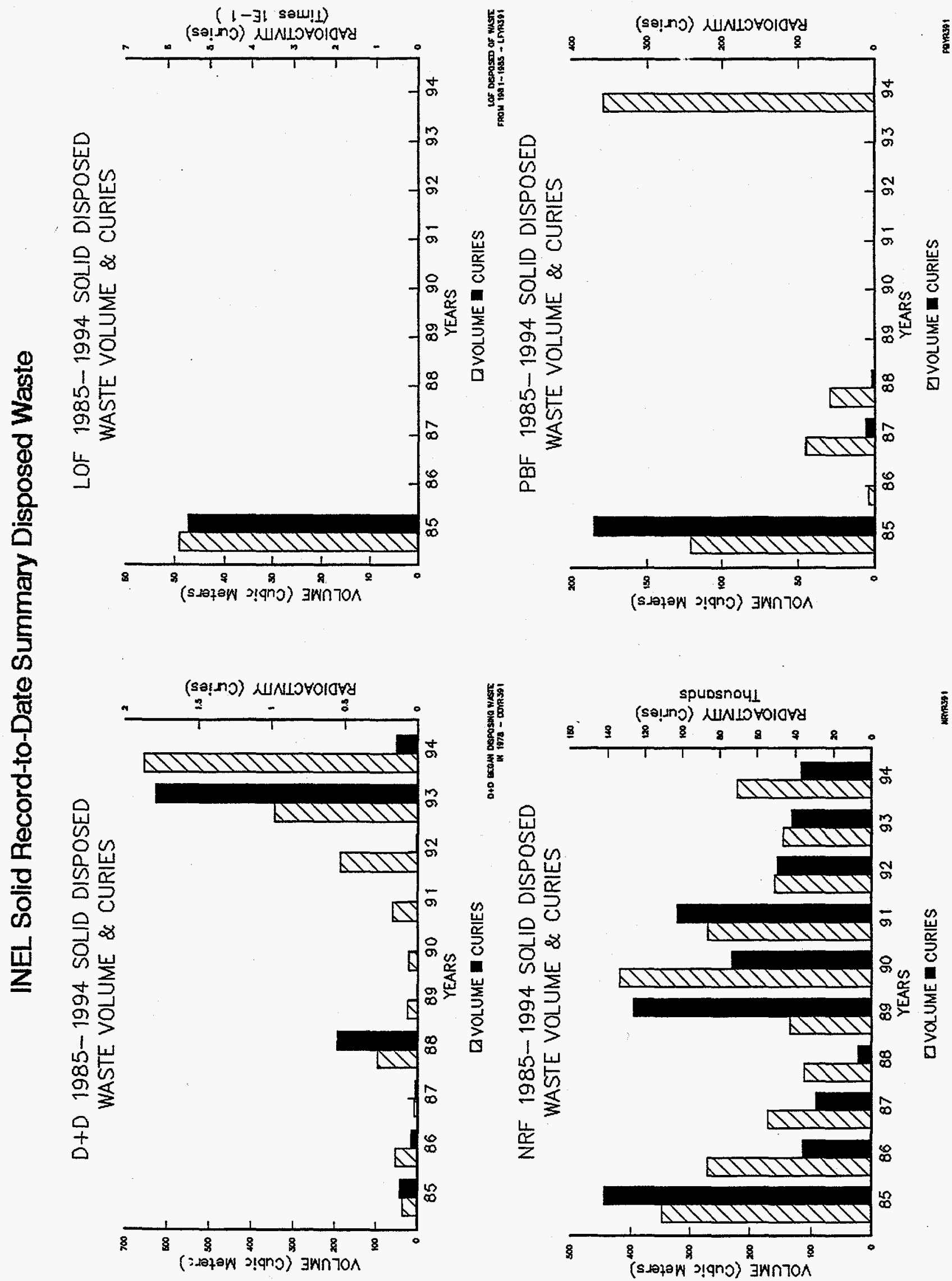

要

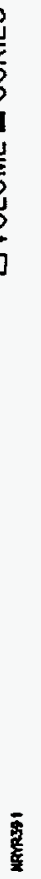

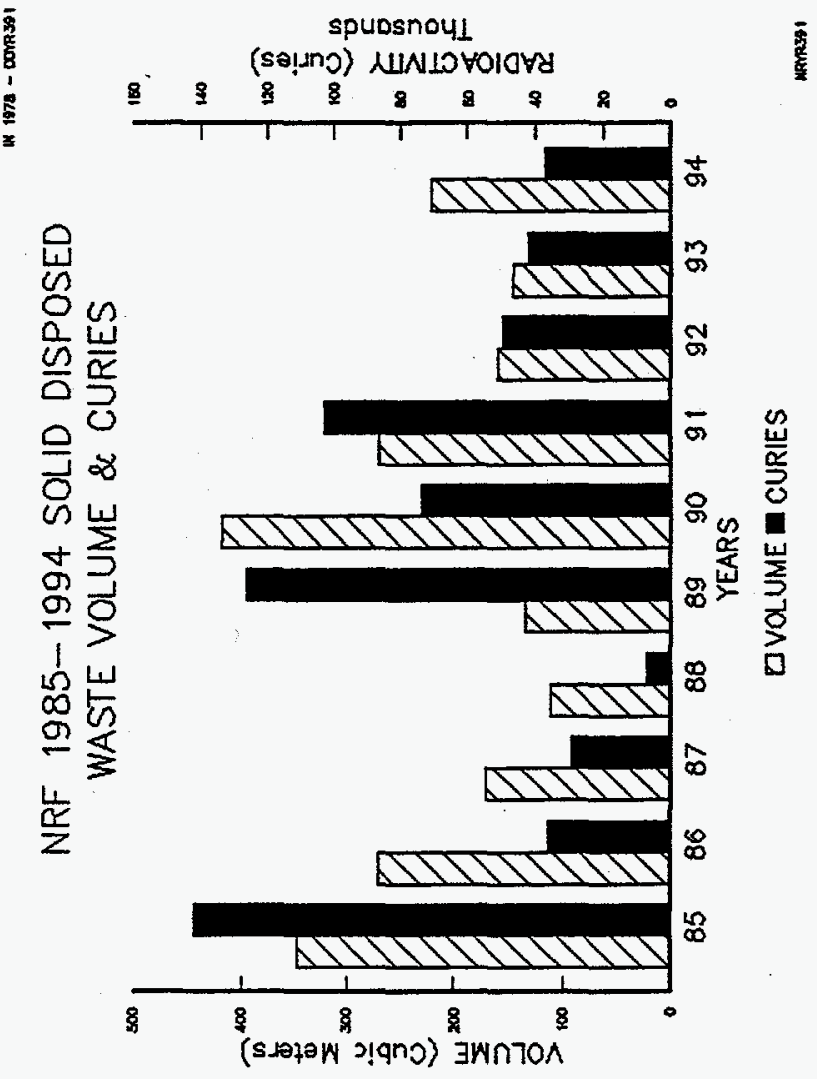

INEL-25 


\section{INEL Solid Record-to-Date Summary Disposed Waste}

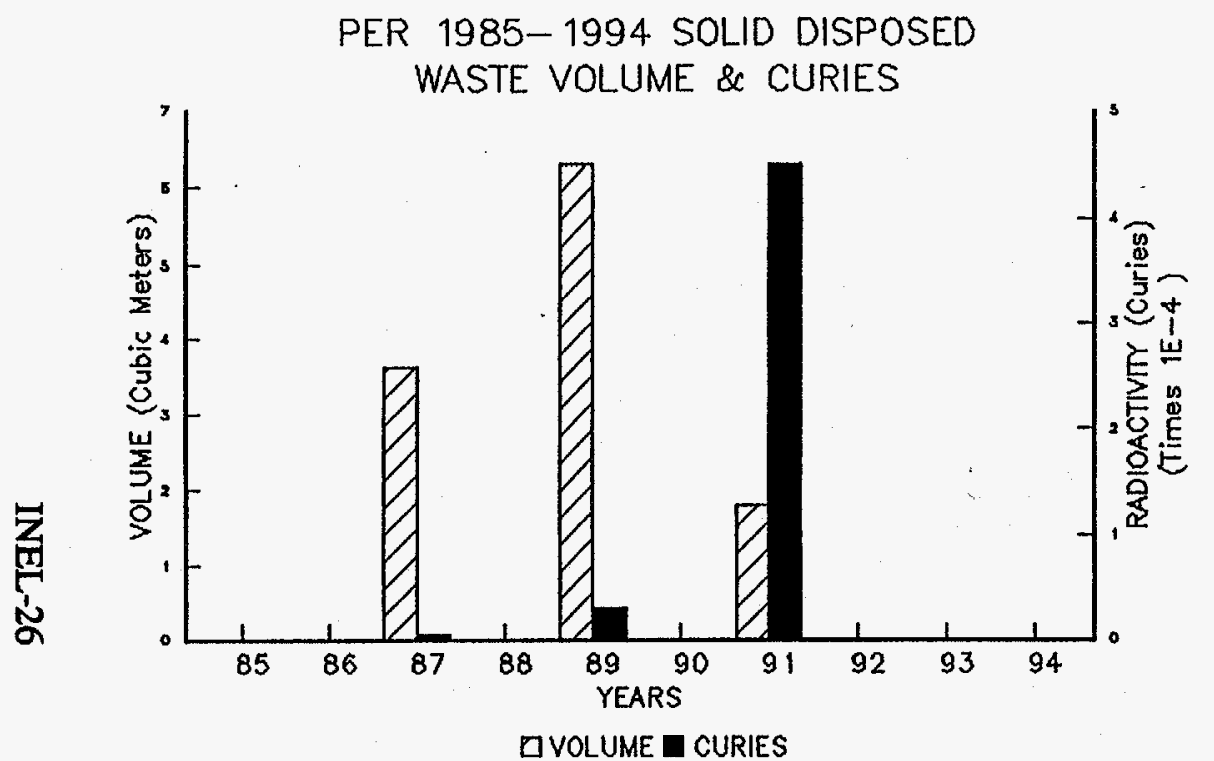

QVOLUME C CURIES

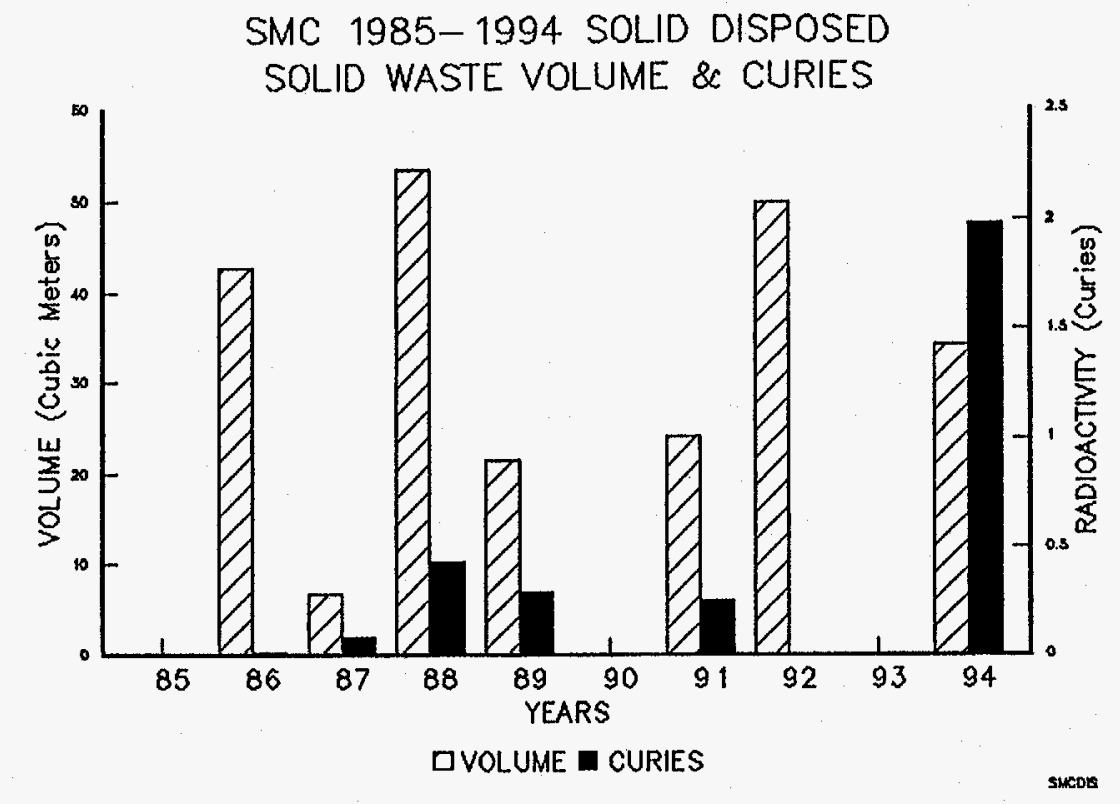

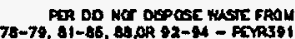

SMC 1985-1994 SOLID DISPOSED

TAN 1985-1994 SOLID DISPOSED WASTE VOLUME \& CURIES

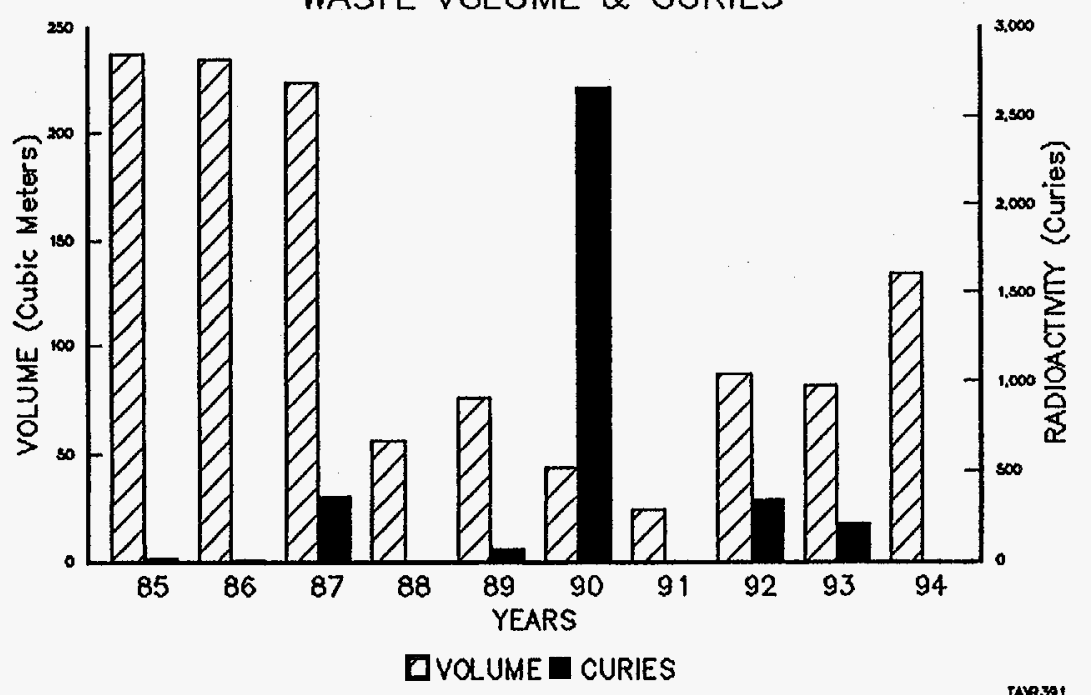




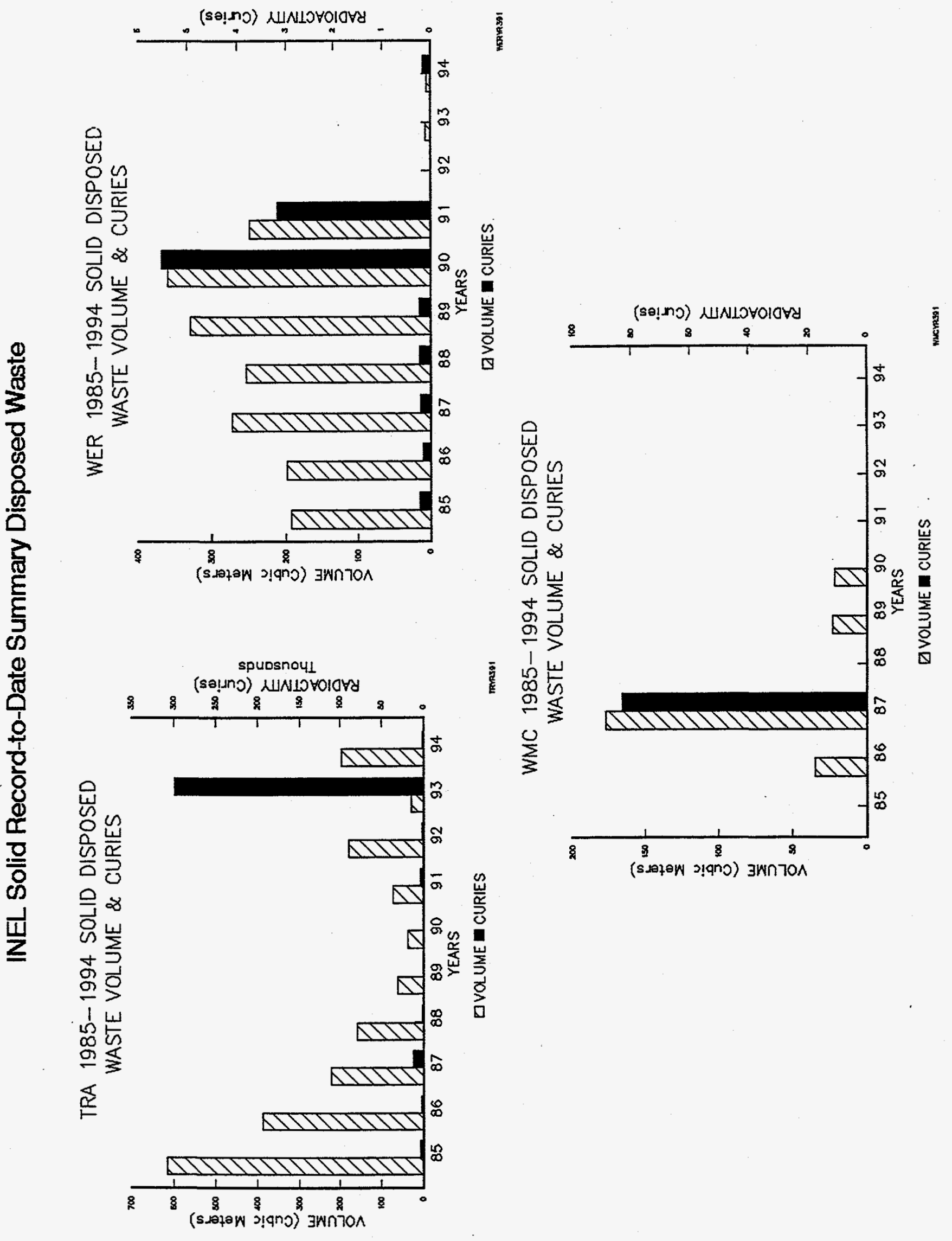

INEL-27 
STORED MASTE

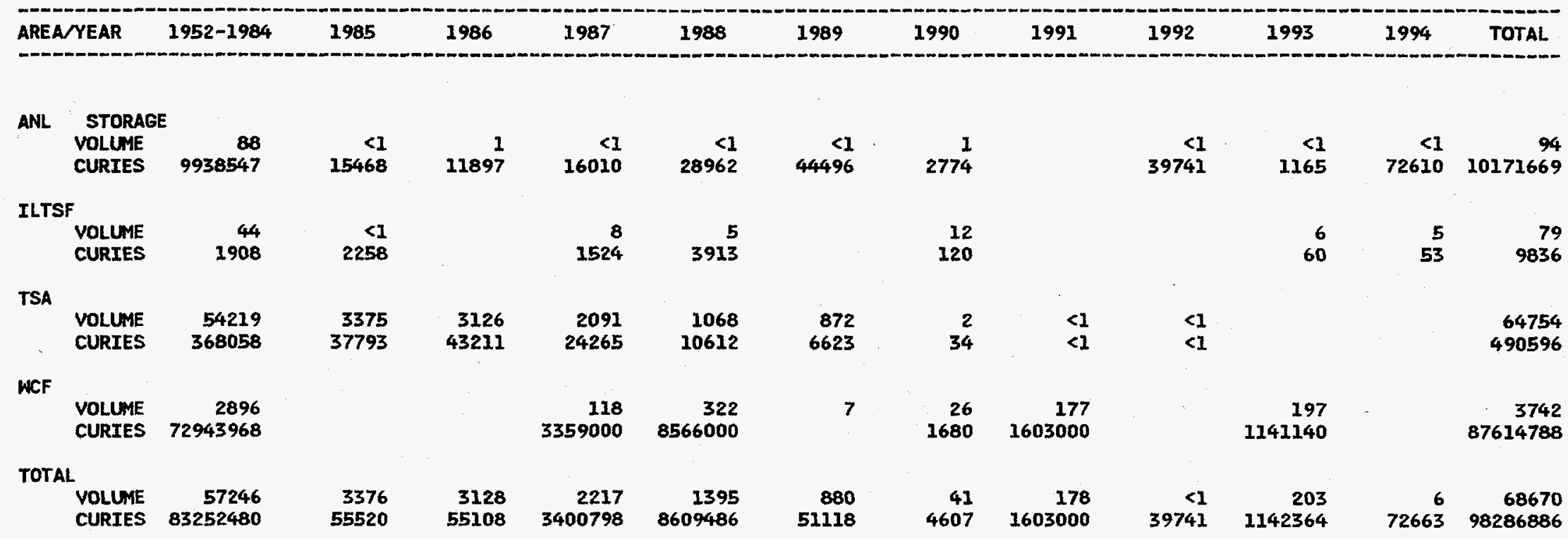

VOLUME IN CUBIC METERS 


\section{INEL Record-to-Date Summary Stored Waste}

1985- 1994 ANL STORAGE AREA

SOLID STORED WASTE VOLUME \& CURIES

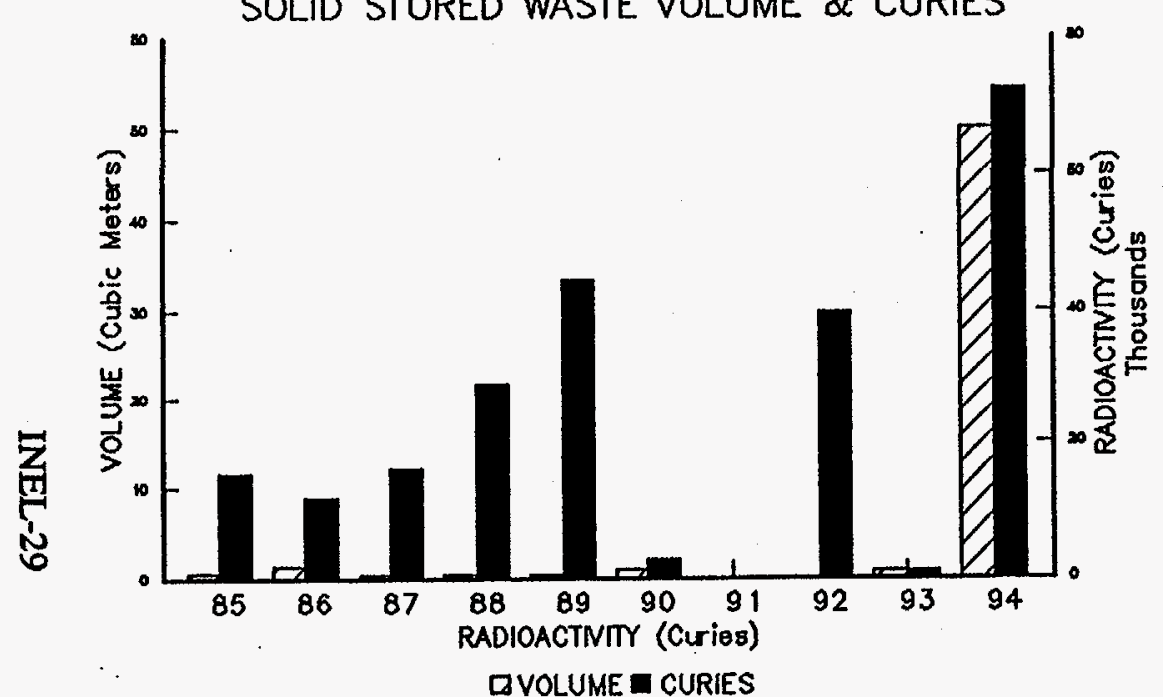

1985-1994 TRANSURANIC STORAGE AREA (TSA) SOLID STORED WASTE VOLUME \& CURIES

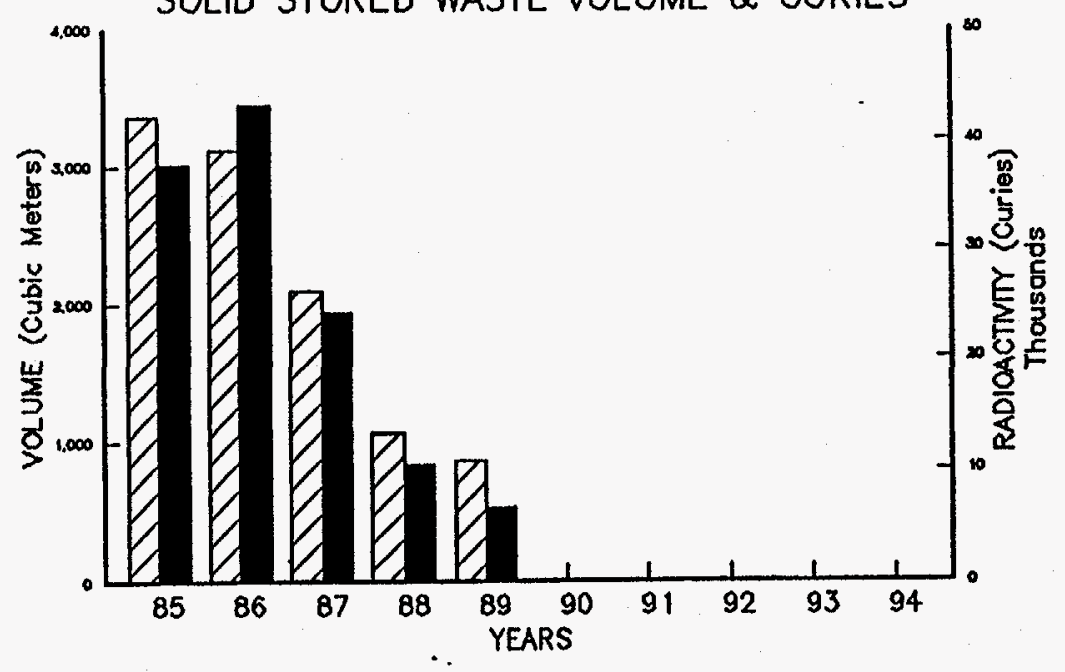

1985- 1994 INTERMEDIATE LEVEL TRANSURANIC STORAGE FACILITY

SOLID STORED WASTE VOLUME \& CURIES

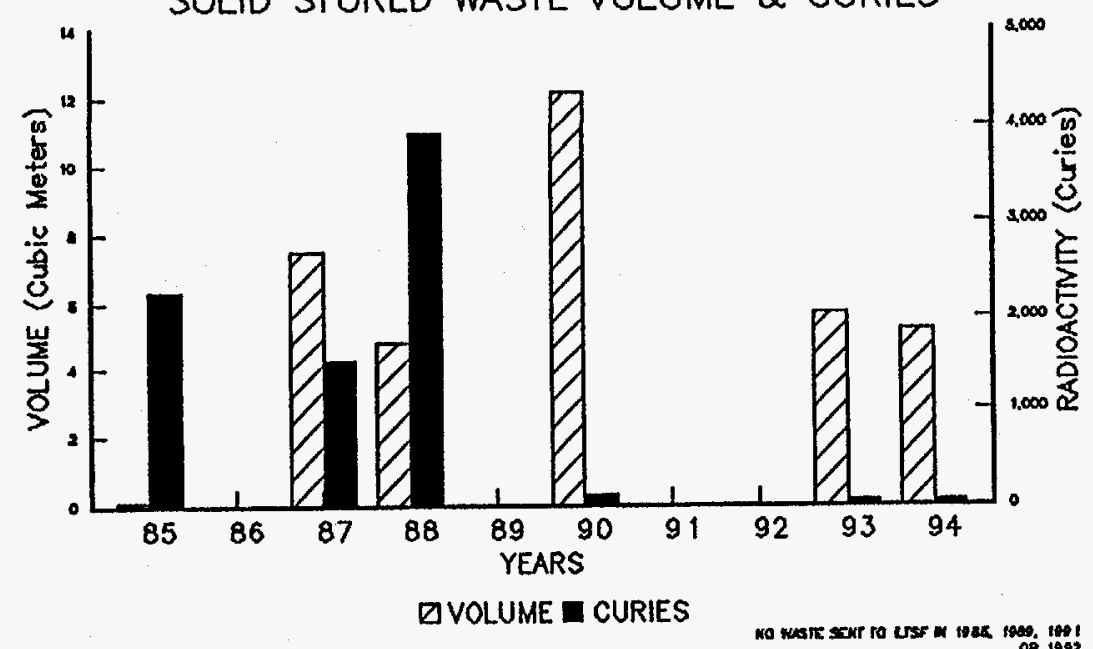

1985-1994 WASTE CALCINING FACILITY

SOLID STORED WASTE VOLUME \& CURIES

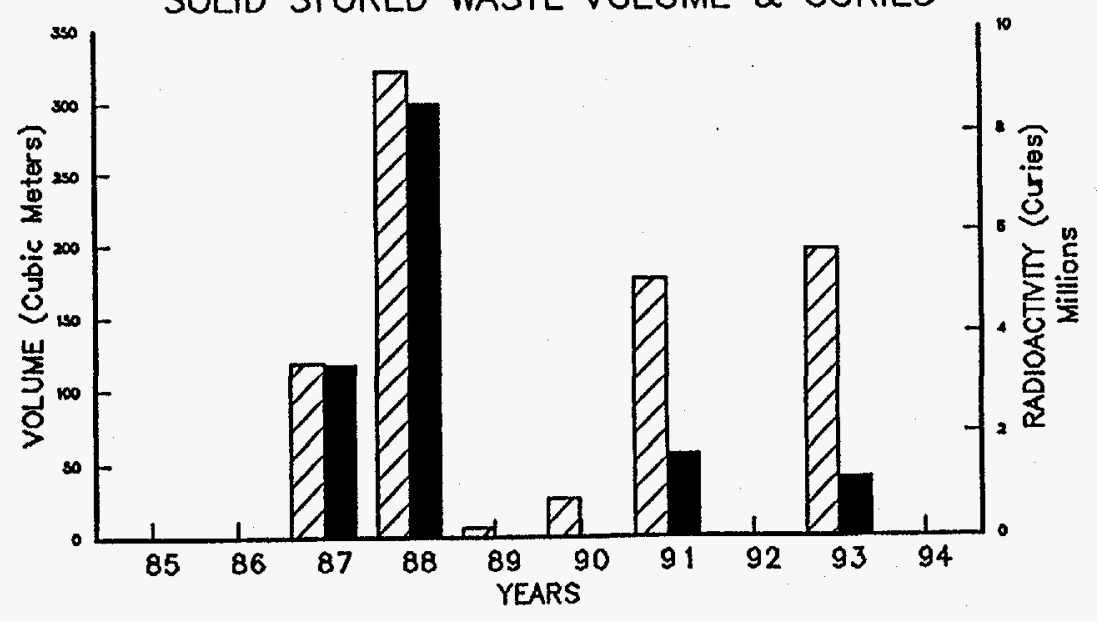

D YOLUME CURIES 
ANL STORAGE WASTE

\begin{tabular}{|c|c|c|c|c|c|c|c|c|c|c|c|c|}
\hline AREA YEAR & 1952-1984 & 1985 & 1986 & 1987 & 1988 & 2989 & 1990 & 1991 & 1992 & 1993 & 2994 & TOTAL \\
\hline \multicolumn{13}{|l|}{ ANL } \\
\hline $\begin{array}{l}\text { VOLUME } \\
\text { CURIES }\end{array}$ & $\begin{array}{r}87 \\
9938491\end{array}$ & $\begin{array}{r}<1 \\
15468\end{array}$ & 11897 & $\begin{array}{r}<1 \\
16010\end{array}$ & $\begin{array}{r}<1 \\
28962\end{array}$ & $\begin{array}{r}<1 \\
44496\end{array}$ & $\begin{array}{r}1 \\
2774\end{array}$ & & $\begin{array}{r}<1 \\
39741\end{array}$ & $\begin{array}{r}<1 \\
2165\end{array}$ & $\begin{array}{r}<1 \\
72620\end{array}$ & $\begin{array}{r}93 \\
10171613\end{array}$ \\
\hline \multicolumn{13}{|l|}{ TRA } \\
\hline $\begin{array}{l}\text { VOLUME } \\
\text { CURIES }\end{array}$ & $\begin{array}{l}<1 \\
<1\end{array}$ & & & & & & & & & & & $\begin{array}{l}<1 \\
<1\end{array}$ \\
\hline \multicolumn{13}{|l|}{ TOTAL } \\
\hline $\begin{array}{l}\text { VOLUME } \\
\text { CURIES }\end{array}$ & $\begin{array}{r}88 \\
9938491\end{array}$ & $\begin{array}{r}<1 \\
15468\end{array}$ & $\begin{array}{r}1 \\
11897\end{array}$ & $\begin{array}{r}<1 \\
16010\end{array}$ & $\begin{array}{r}<1 \\
28962\end{array}$ & $\begin{array}{r}<1 \\
44496\end{array}$ & $\begin{array}{r}1 \\
2774\end{array}$ & & $\begin{array}{r}<1 \\
39741\end{array}$ & $\begin{array}{r}<1 \\
1165\end{array}$ & $\begin{array}{r}<1 \\
72610\end{array}$ & $\begin{array}{r}94 \\
10171613\end{array}$ \\
\hline
\end{tabular}

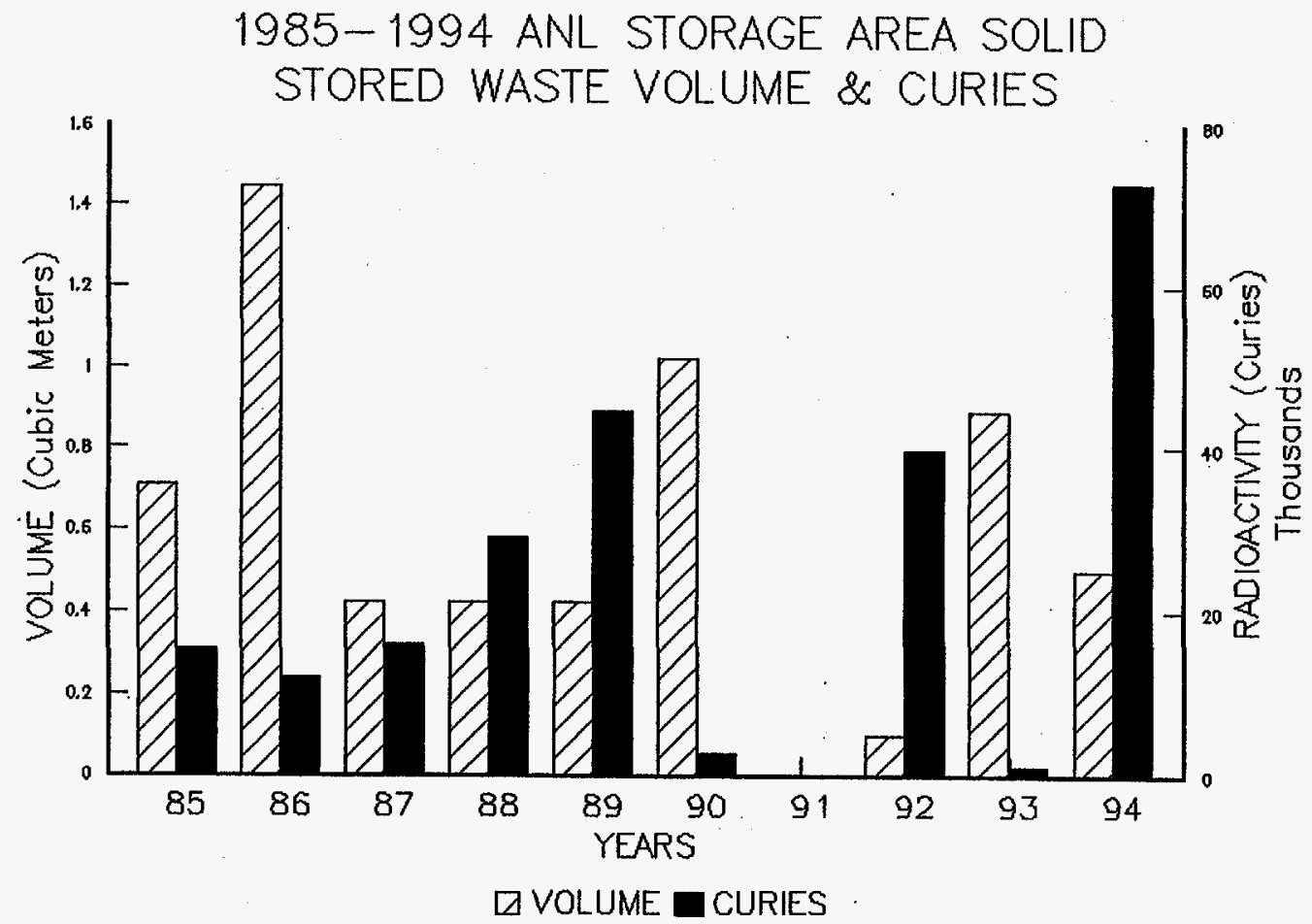


INEL SOLID RECORD-TO-DATE SUMAARY

ILTSF MASTE

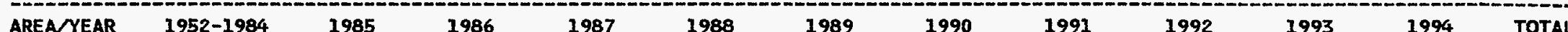

AREAMEAR 1952-1984

1985

1986

1987

1988

1989

1990

1991

1992

1993

TOTAL

ALE

VOLUME $\quad 38$

CURIES 233

$\begin{array}{rr}7 & 3\end{array}$

12

ANL

VOLUME

3

$<1$

4

120

6
60

66
549

$\begin{array}{lll}\text { CURIES } & 1524 & 2258\end{array}$

$\begin{array}{rr}<1 & 1 \\ 1429 & 3268\end{array}$

5
8479

BET

VOLUME

CURIES

$<1$
604

604

言

CPP

VOLUME

$<1$

VOLUME 3

CURIES 81

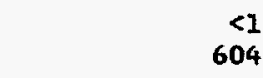

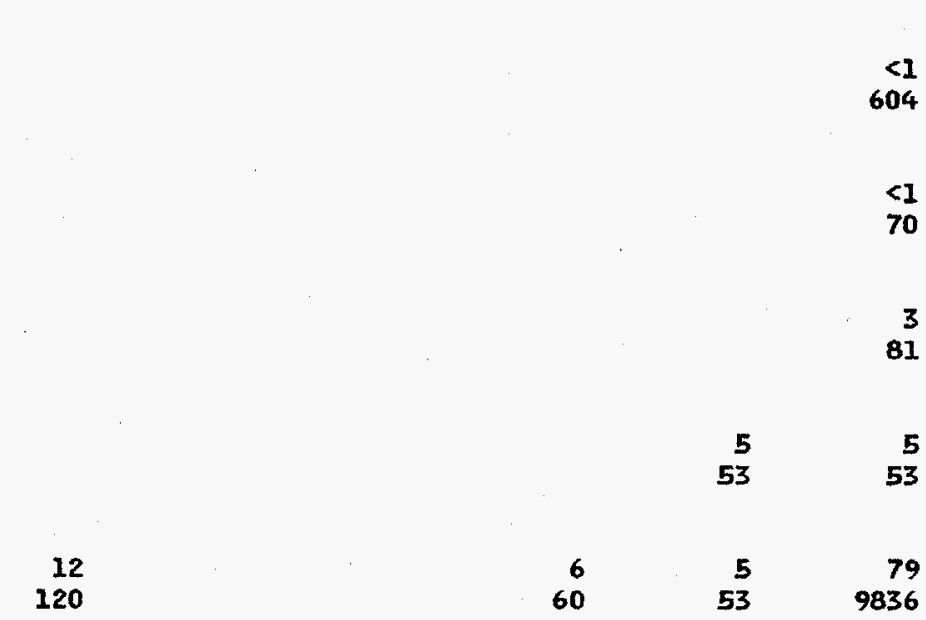

VOLUME

CURIES

$\begin{array}{rr}8 & 5 \\ 1524 & 3913\end{array}$

CURTES

44
1908

$<1$
2258

VOLUME IN CUBIC METERS

DETAILS MAY NOT ADD UP TO TOTALS BECAUSE OF ROUNDING 


\section{INEL Solid Record-to-Date Summary ILTSF Waste}

ALE 1985- 1994 INTERMEDIATE LEVEL TRANSURANIC STORAGE FACILITY SOLID WASTE VOLUME \& CURIES

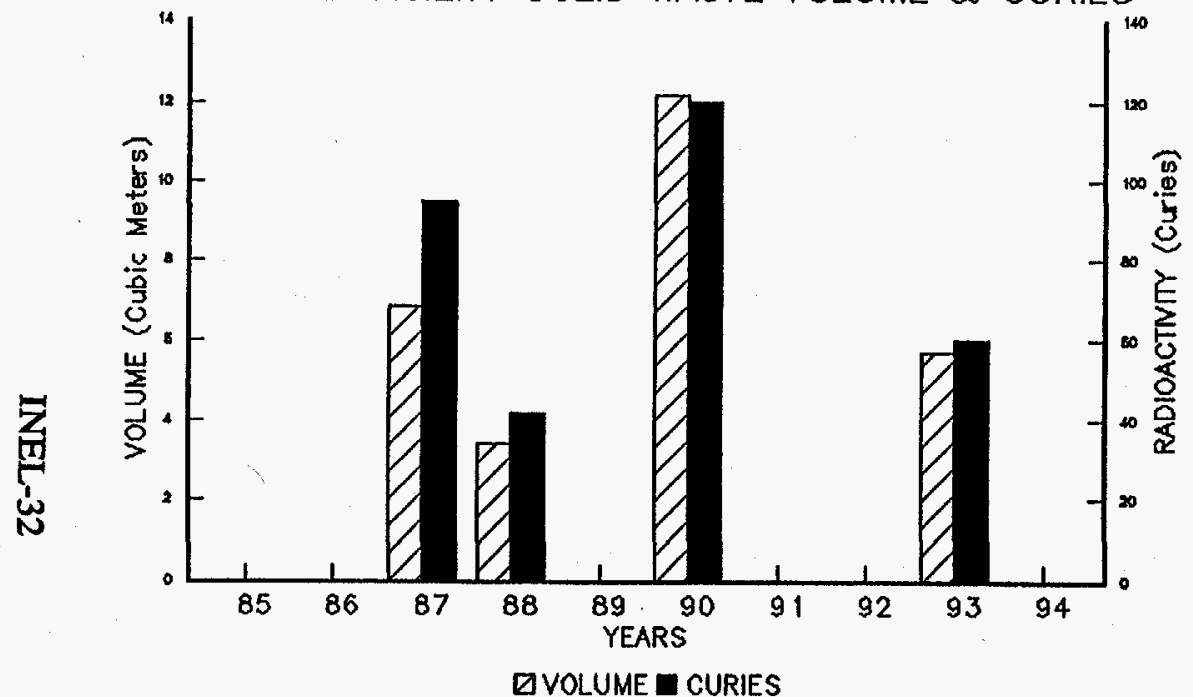

BET 1985- 1994 INTERMEDIATE LEVEL TRANSURANIC STORAGE FACILITY SOLID WASTE VOLUME \& CURIES

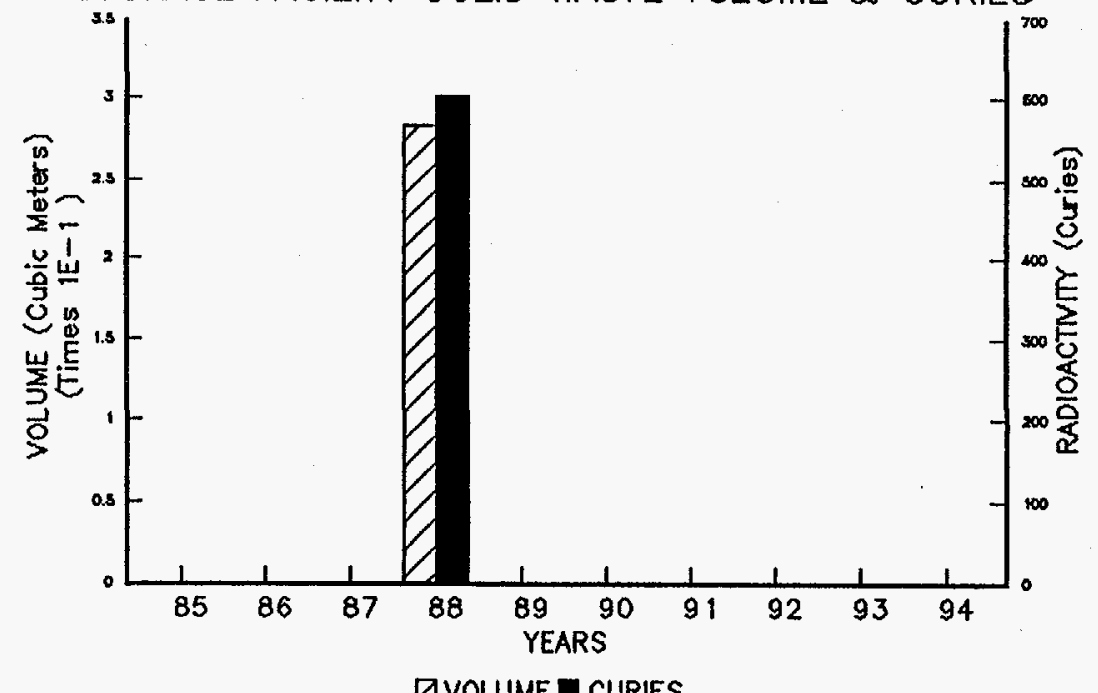

ANL 1985-1994 INTERMEDIATE LEVEL TRANSURANIC STORAGE FACILITY SOLID WASTE VOLUME \& CURIES

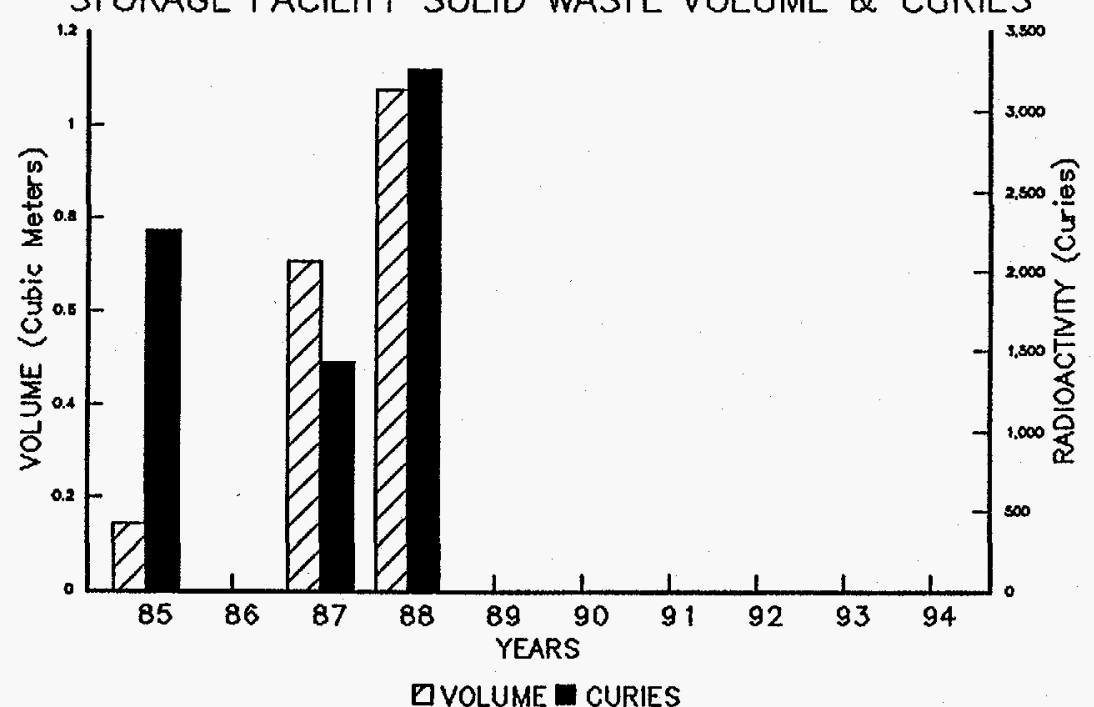

TRA 1985-1994 INTERMEDIATE LEVEL TRANSURANIC STORAGE FACILITY SOLID WASTE VOLUME \& CURIES

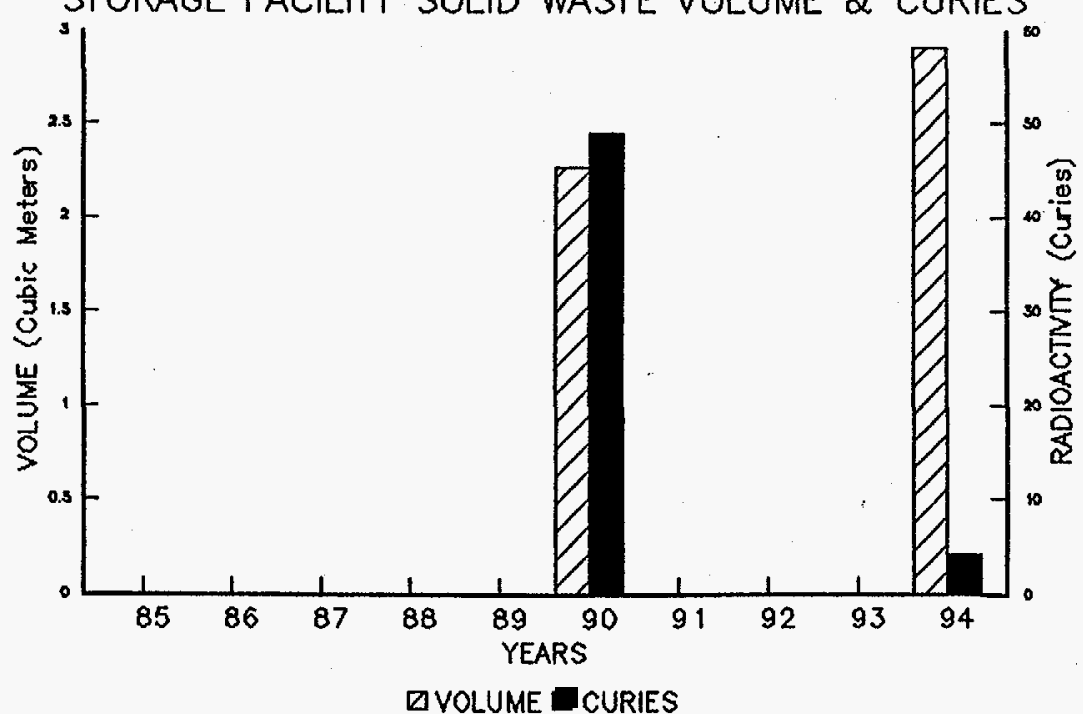


TSA WASTE

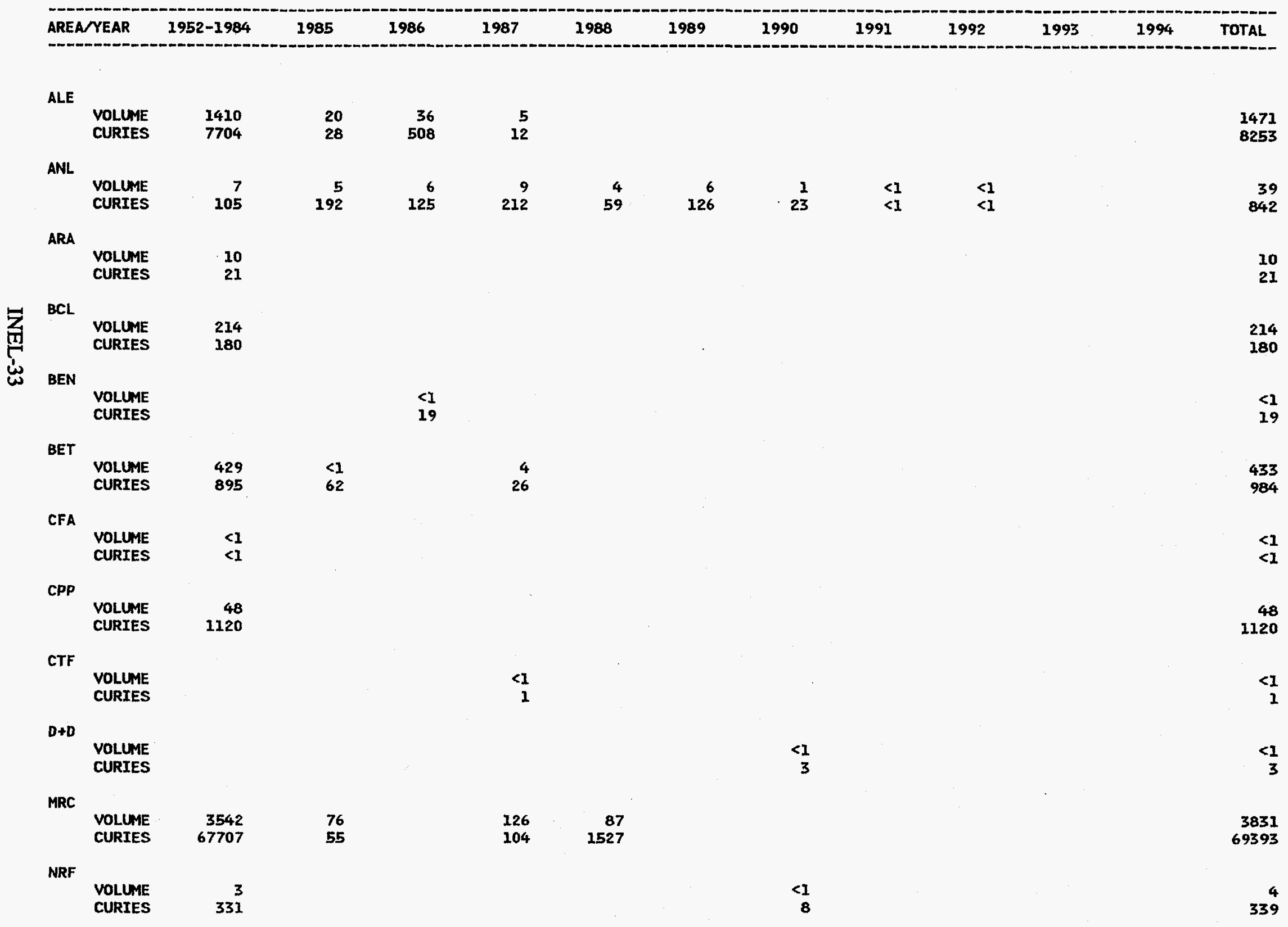


SCHEdULE NO. P61PM022-M

INEL. SOLID RECORD-TO-DATE SUMMARY

TSA WASTE

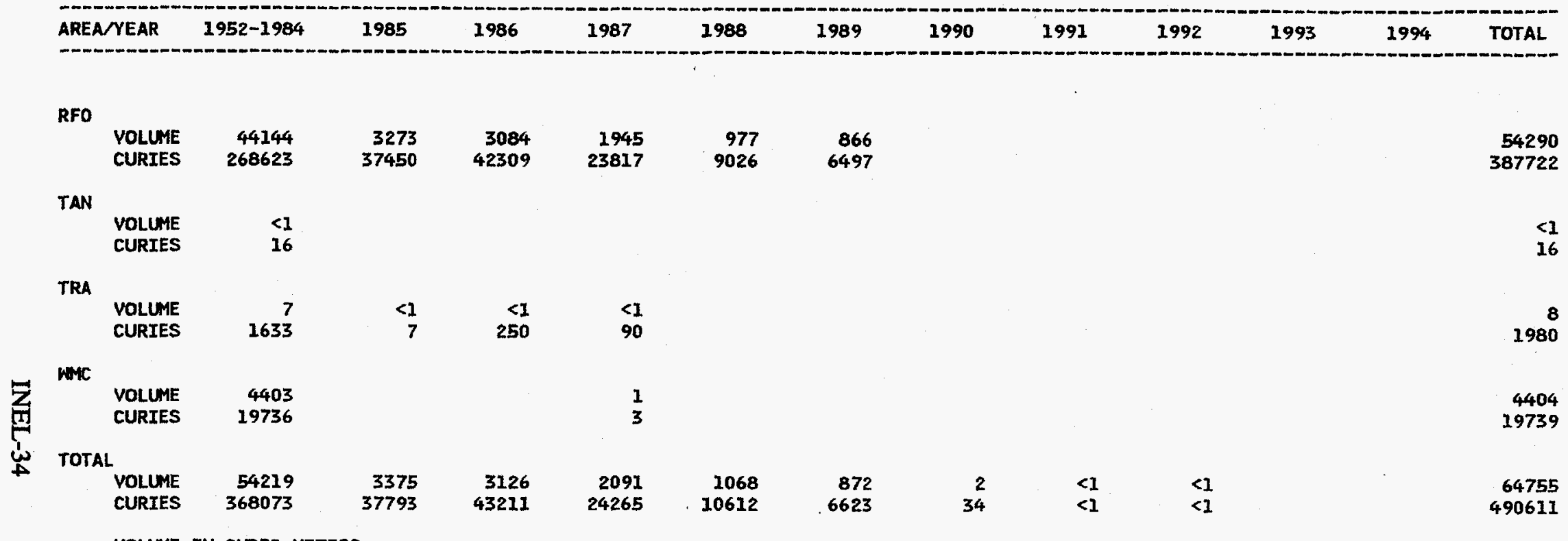

DETAILS MAY NOT ADD UP TO TOTALS BECAUSE OF ROUNDTNG 


\section{INEL Solid Record-to-Date Summary TSA Waste}

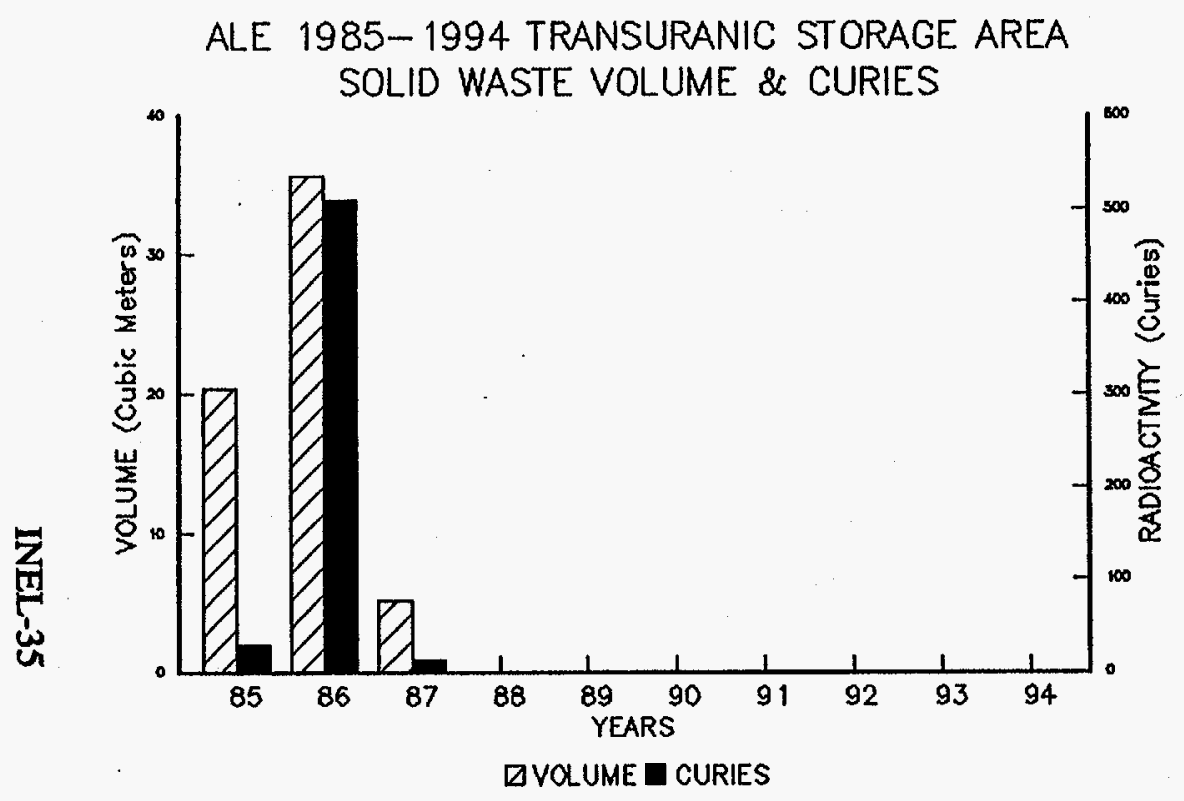

BEN 1985-1994 TRANSURANIC STORAGE AREA

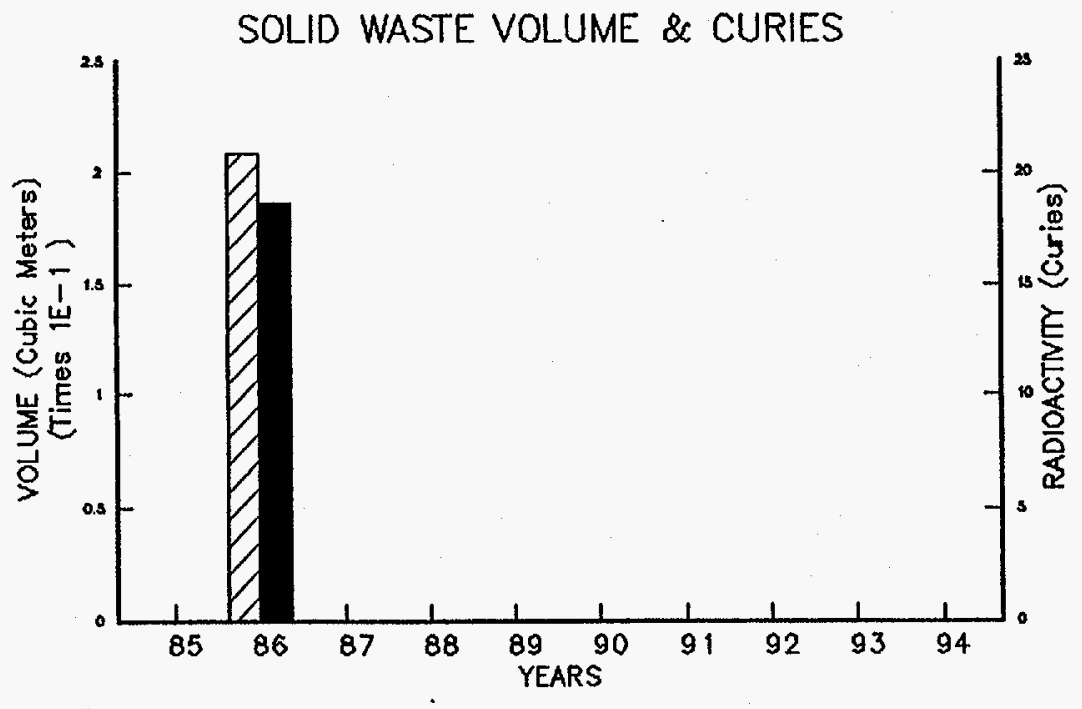

CVYLUME I CURIES
ANL 1985-1994 TRANSURANIC STORAGE AREA SOLID WASTE VOLUME \& CURIES

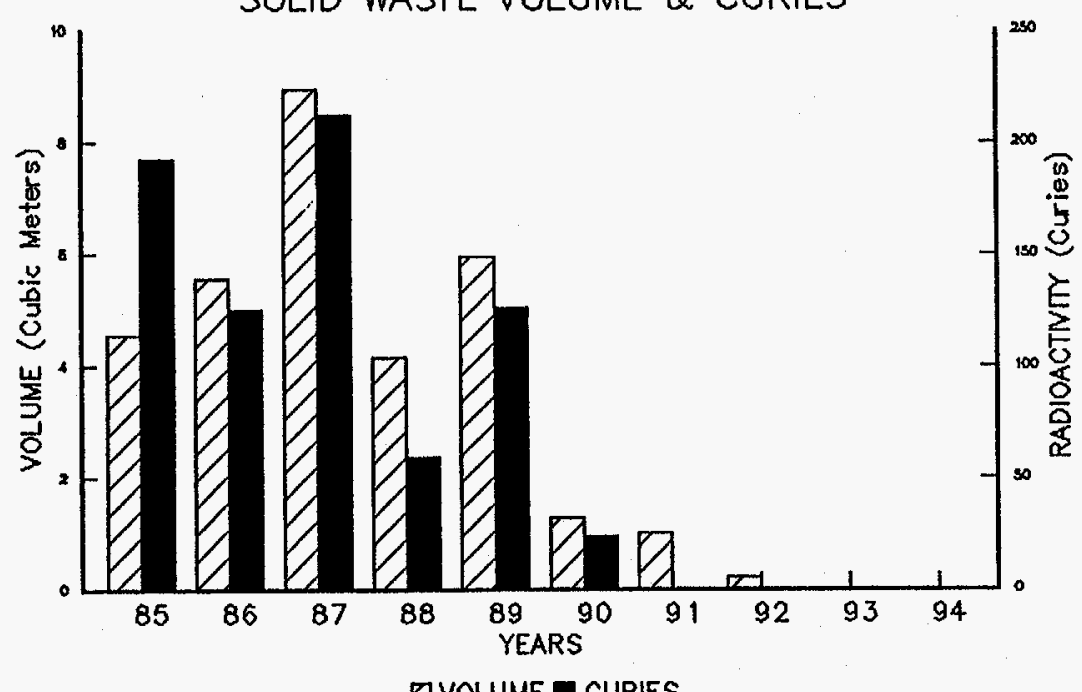

DVOLUME C CURIES

BET 1985-1995 TRANSURANIC STORAGE AREA SOLID WASTE VOLUME \& CURIES

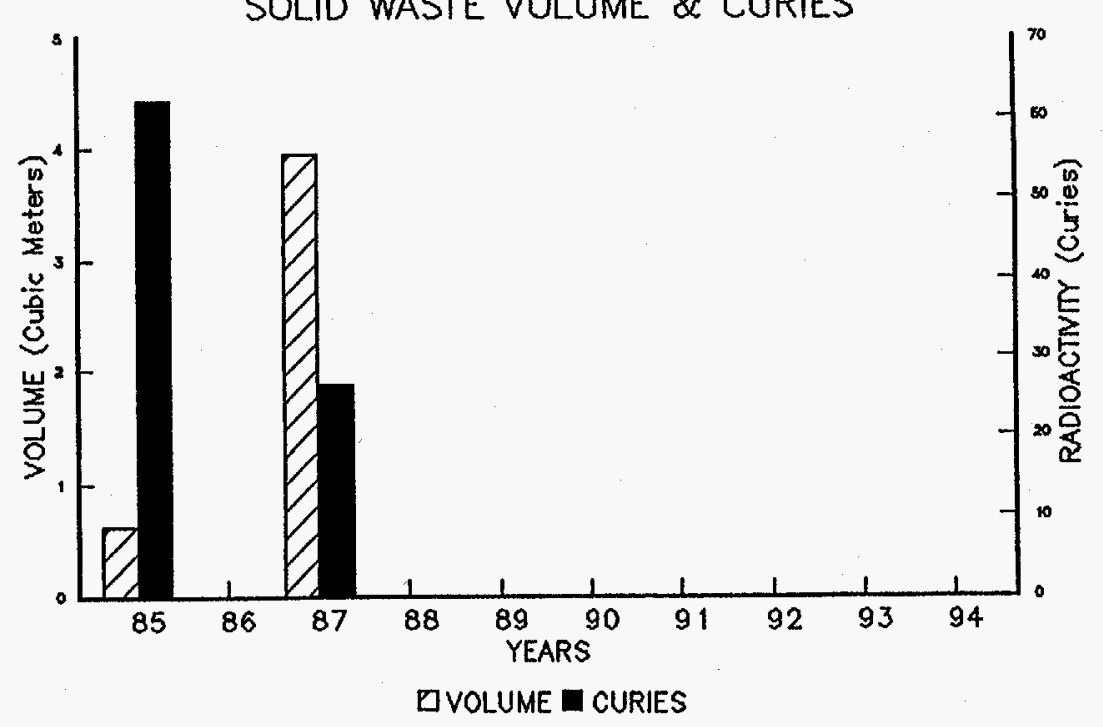




\section{INEL Solid Record-to-Date Summary TSA Waste}

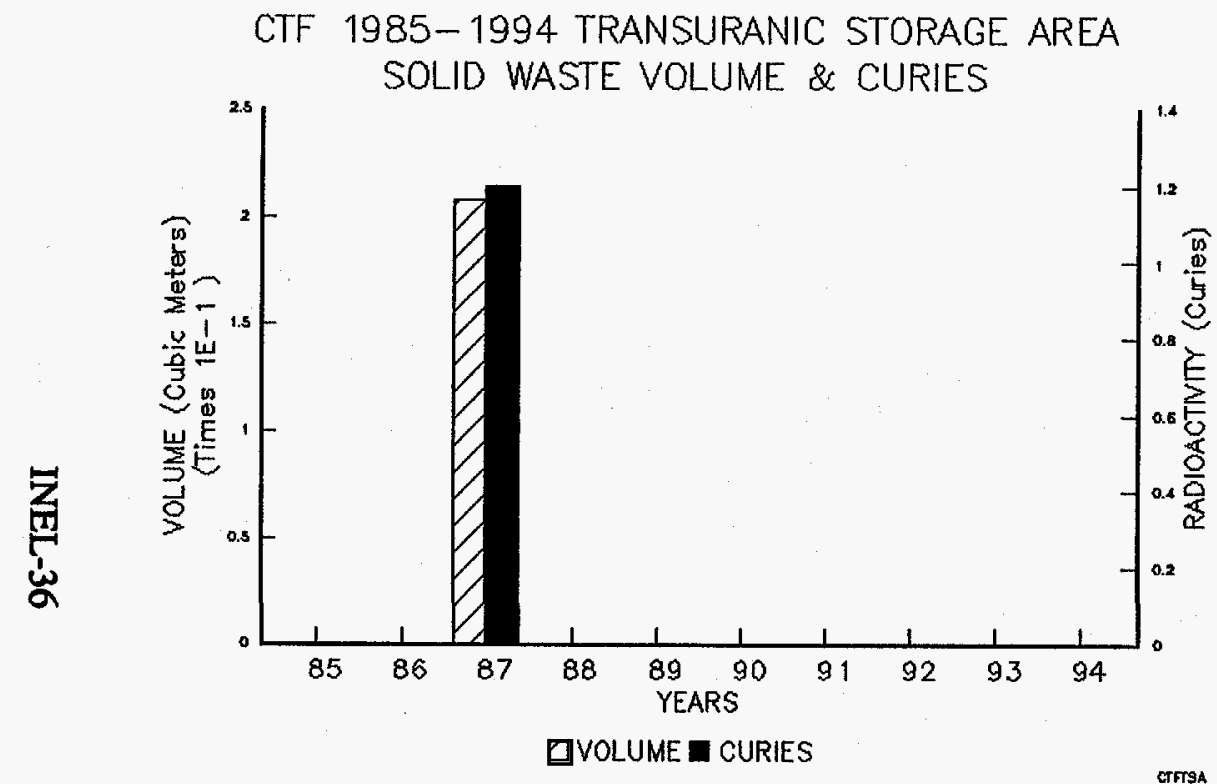

MRC 1985-1994 TRANSURANIC STORAGE AREA

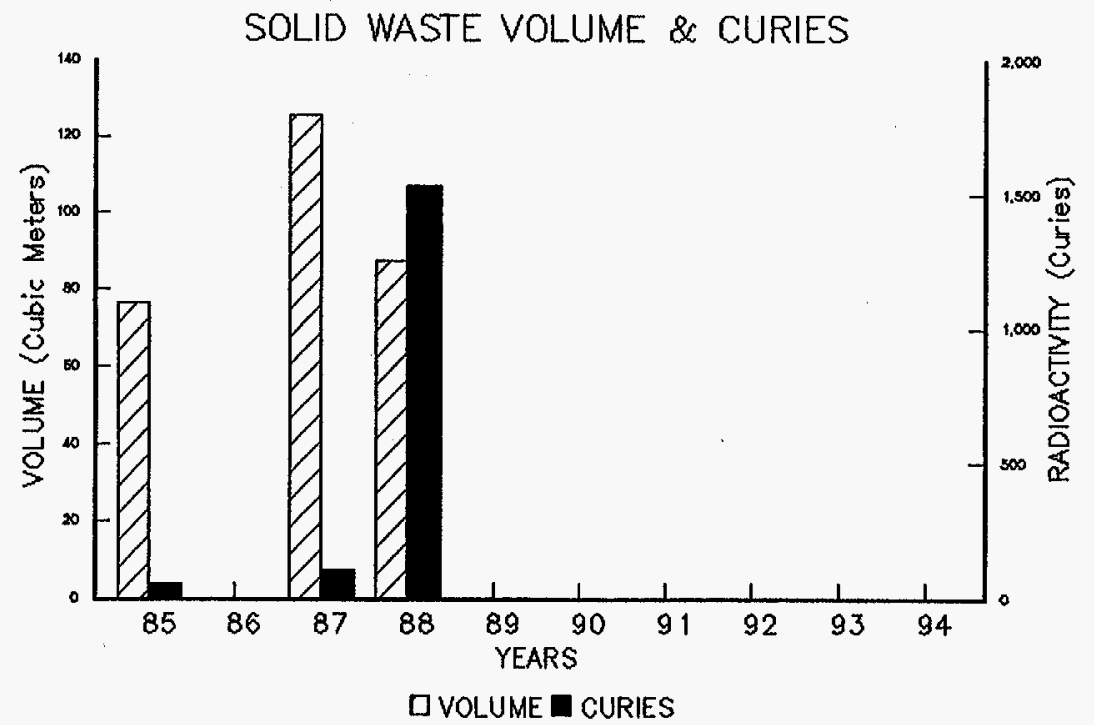

D\&D 1985-1994 TRANSURANIC STORAGE AREA SOLID WASTE VOLUME \& CURIES

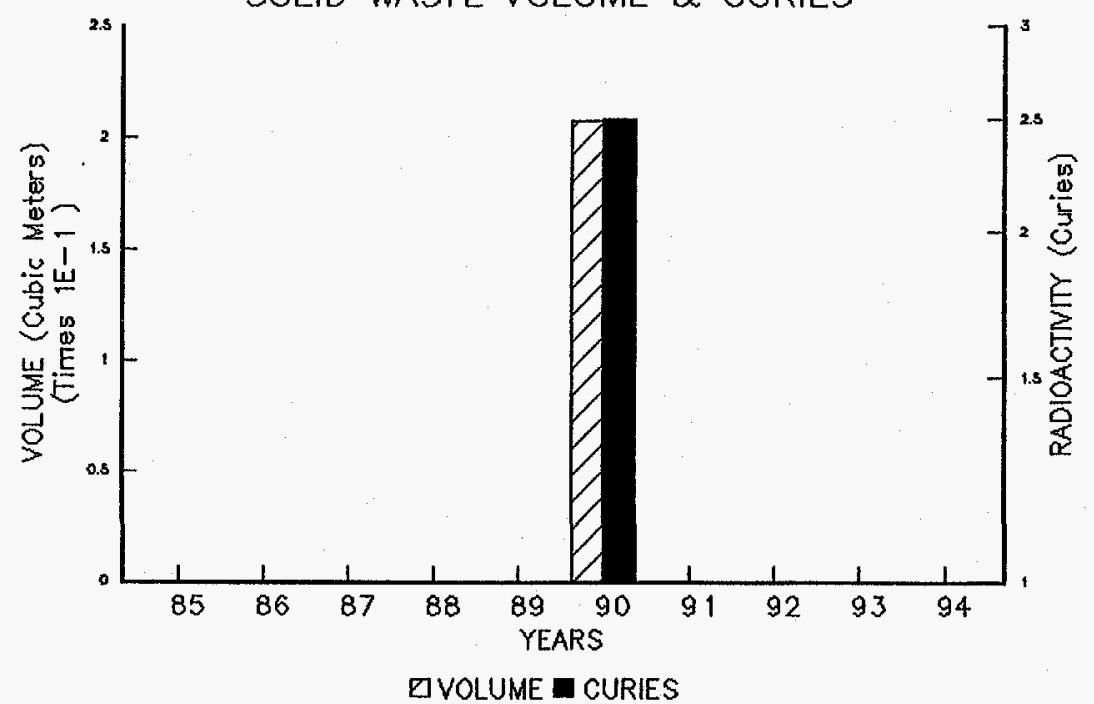

NRF 1985-1994 TRANSURANIC STORAGE AREA

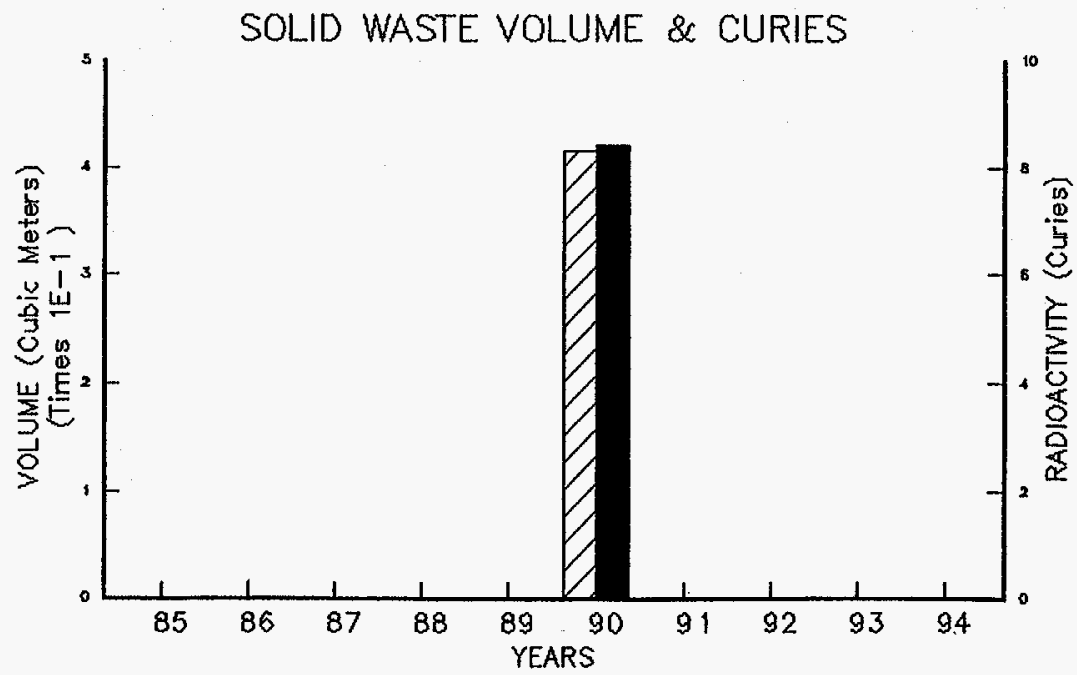

DVOLUME M CURIES 


\section{INEL Solid Record-to-Date Summary TSA Waste}
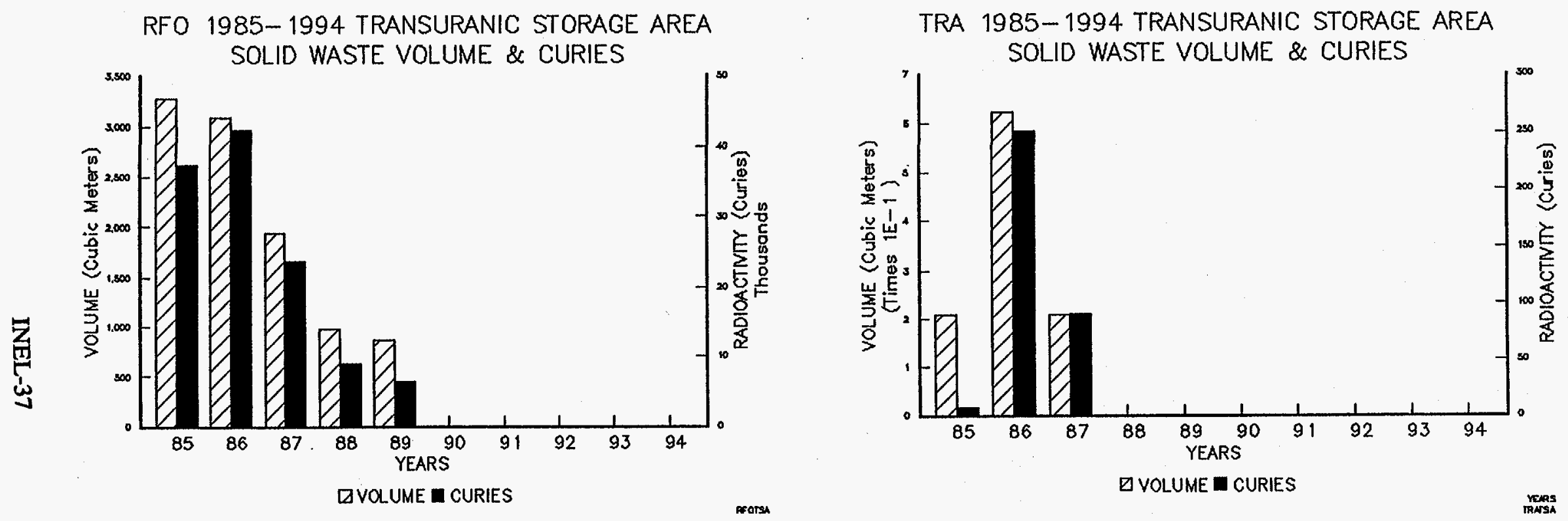

WMC 1985-1994 TRANSURANIC STORAGE AREA

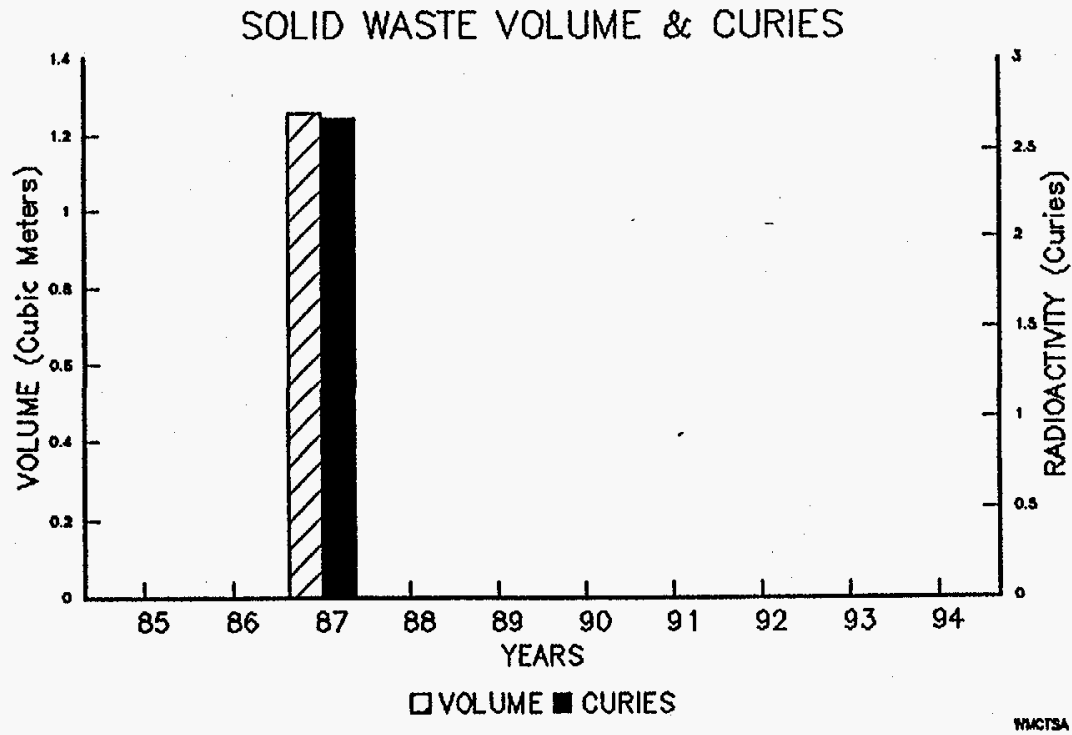


SCHEDULE NO. P61PM022-F

CPP HIGH-LEVEL. LIQUID/SOLID WASTE STORAGE

RUN DATE: 05/24/95 RECORD-TO-DATE

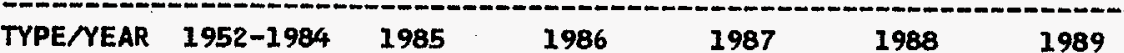

$-1$

1992

LIQUID WASTE TO LIQUID WASTE TANKS

IQUID WASTE TO LIQUTD
VOLUME 26594064

CURIES $\quad 378679040$

1991

1992

1993

1994

TOTAL

LIQUID WASTE TO CALCINER

VOLUME 46126432

CALCINED SOLIDS TO STORAGE

VOLUME 2896

CURIES $\quad 72944064$

$$
72944064
$$

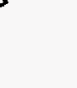

$\begin{array}{rrrrrrr} & & & & \begin{array}{r}26594064 \\ 378679040\end{array} \\ 856257 & 2275040 & & 181700 & 1627800 & 1139800 & 52207024 \\ 118 & & & & & & \\ 3359000 & 8566000 & 7 & 1680 & 1603000 & 177 & 3742 \\ & & & & & 1141140 & 87614880\end{array}$

LIQUID VOLUME IN LITERS

TI SOLID VOLUME IN CUBIC METERS

心 LIQUID WASTES WERE GENERATED BEGINWING IN 1953,

HOWEVER, THE WASTE CALCINATION PROCESS DID NOT BEGIN UNTIL 1963.

DETAILS MAY NOT ADD UP TO TOTALS BECAUSE OF ROUNDING 

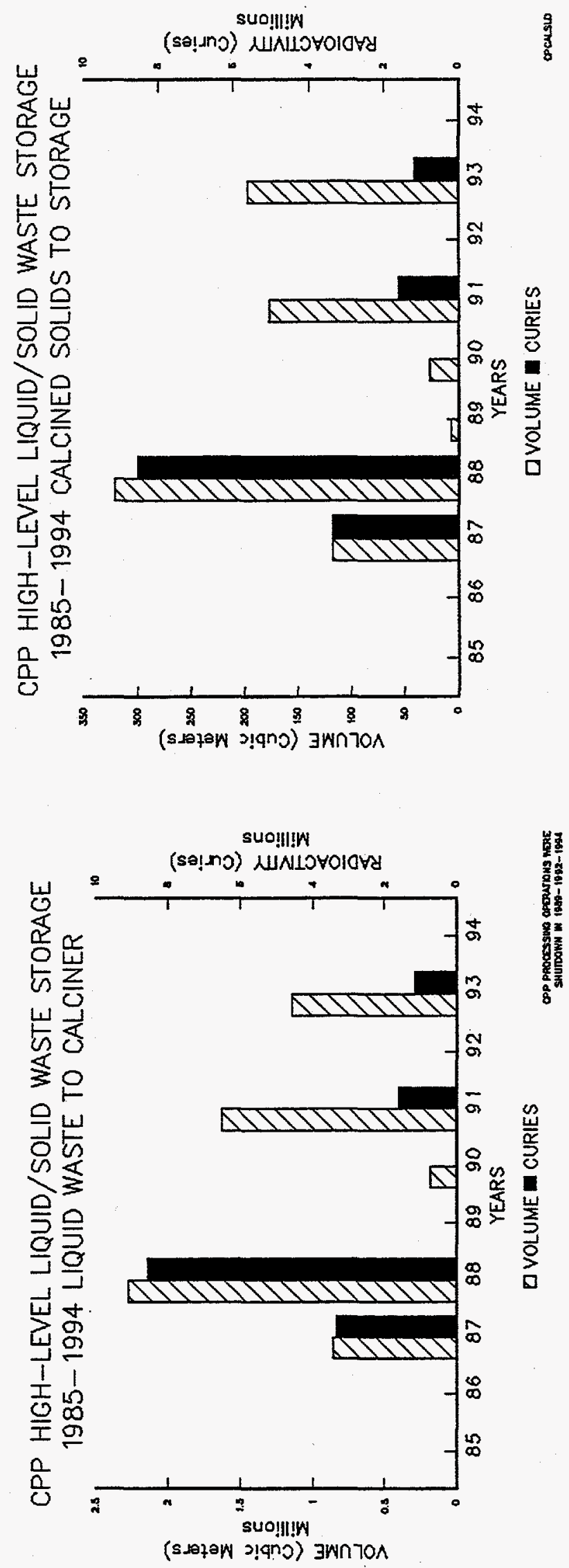

INEL-39 
WERF MASTE

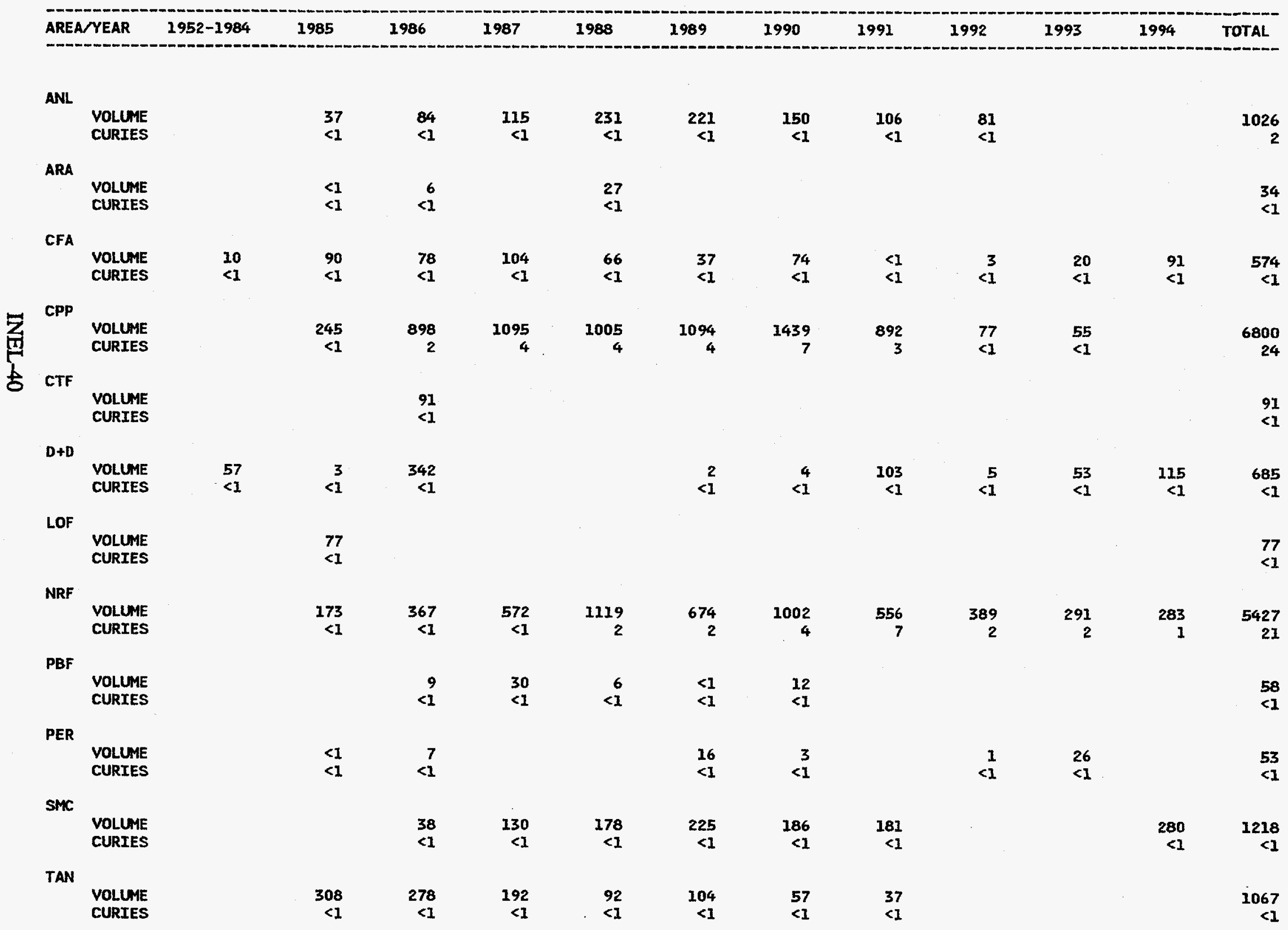


INEL SOLID RECORD-TO-DATE SUMMARY

WERF WASTE

\begin{tabular}{|c|c|c|c|c|c|c|c|c|c|c|}
\hline AREA YEAR & $1952-1984$ & 1985 & 1986 & 1987 & 1988 & 1989 & 1990 & 1991 & 1992 & TOTAL \\
\hline
\end{tabular}

TRA

\begin{tabular}{|c|c|c|c|c|c|c|c|c|c|c|c|c|}
\hline VOLUME & 11 & 340 & 257 & 286 & 244 & 290 & 227 & 147 & 384 & & & 2185 \\
\hline CURIES & $<1$ & $<1$ & $<1$ & $<1$ & $<1$ & $<1$ & $<1$ & 5 & 11 & & & 17 \\
\hline $\begin{array}{l}\text { VOLUME } \\
\text { CURIES }\end{array}$ & & & $\begin{array}{r}2 \\
<1\end{array}$ & $\begin{array}{r}4 \\
<1\end{array}$ & & & $\begin{array}{r}6 \\
<1\end{array}$ & $\begin{array}{r}6 \\
<1\end{array}$ & & & & $\begin{array}{l}19 \\
<1\end{array}$ \\
\hline $\begin{array}{l}\text { VOLUME } \\
\text { CURIES }\end{array}$ & $\begin{array}{l}78 \\
<1\end{array}$ & $\begin{array}{r}1275 \\
1\end{array}$ & $\begin{array}{r}2458 \\
2\end{array}$ & $\begin{array}{r}2529 \\
5\end{array}$ & $\begin{array}{r}2967 \\
6\end{array}$ & $\begin{array}{r}2663 \\
6\end{array}$ & $\begin{array}{r}3161 \\
12\end{array}$ & $\begin{array}{r}2029 \\
15\end{array}$ & $\begin{array}{r}940 \\
13\end{array}$ & $\begin{array}{r}446 \\
2\end{array}$ & $\begin{array}{r}769 \\
1\end{array}$ & $\begin{array}{r}19315 \\
65\end{array}$ \\
\hline
\end{tabular}

点

DETAILS MAY NOT ADD UP TO TOTALS BECAUSE OF ROUNDING 


\section{INEL Solid Record-to-date Summary WERF Waste}
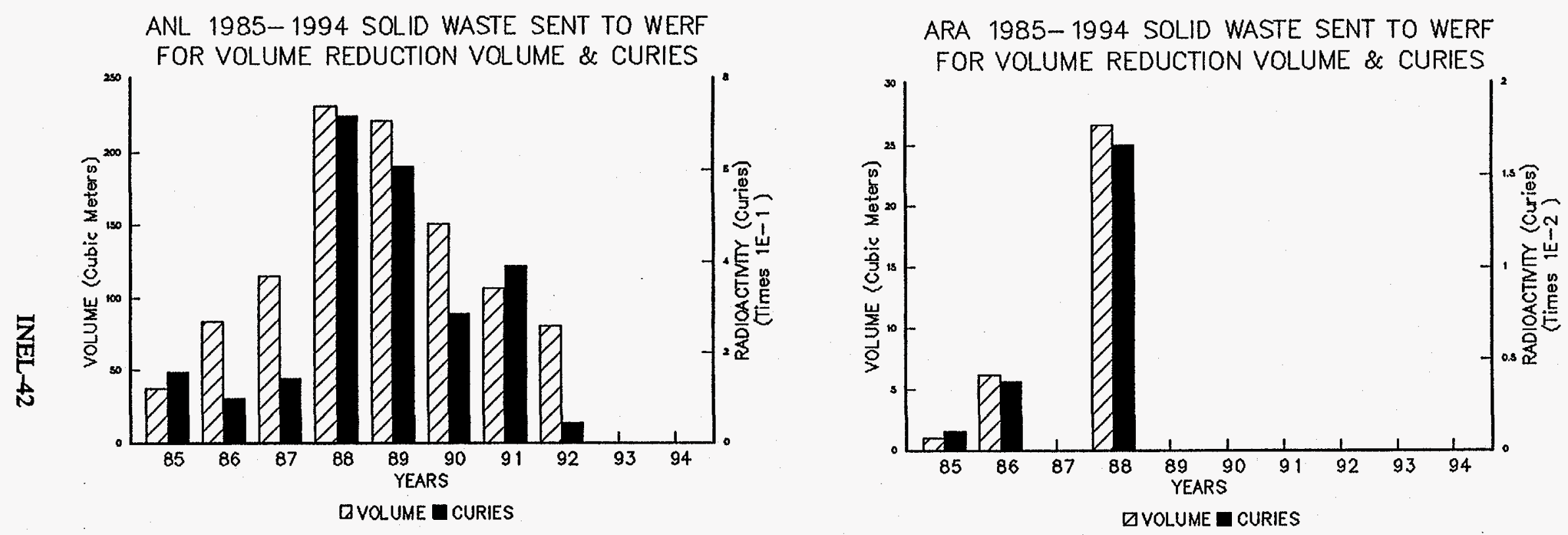

CFA 1985-1994 SOLID WASTE SENT TO WERF FOR VOLUME REDUCTION VOLUME \& CURIES
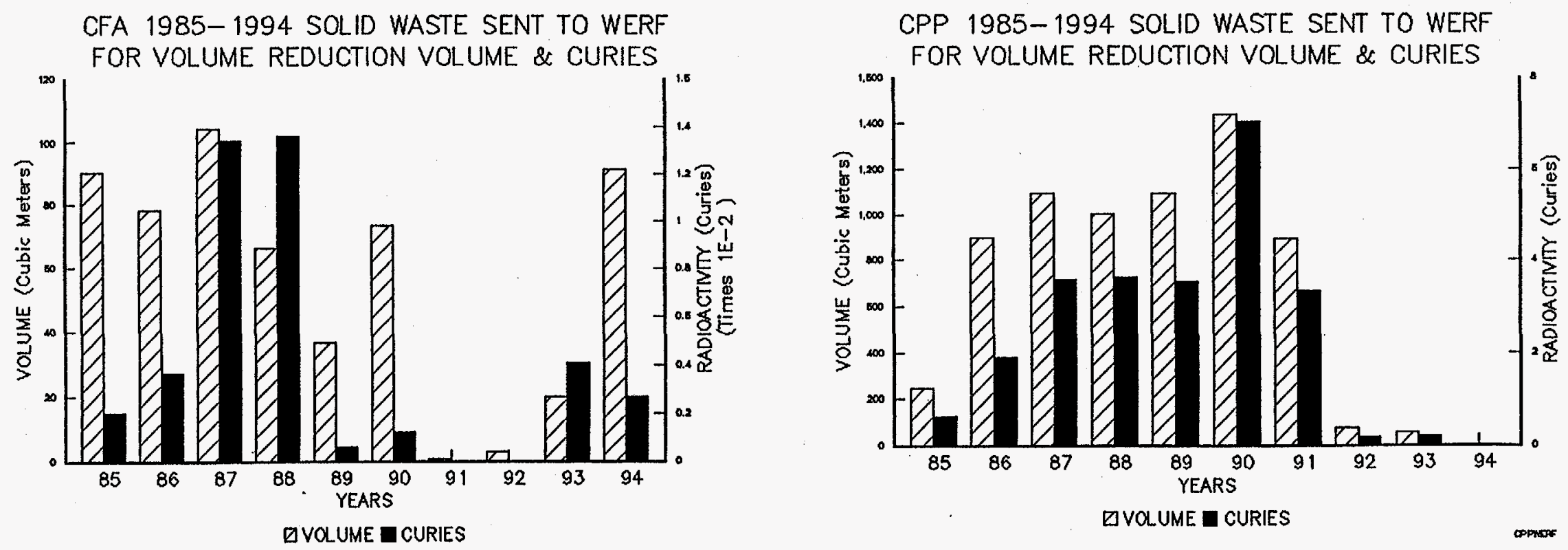


\section{INEL Solid Record-to-Date Summary WERF Waste}
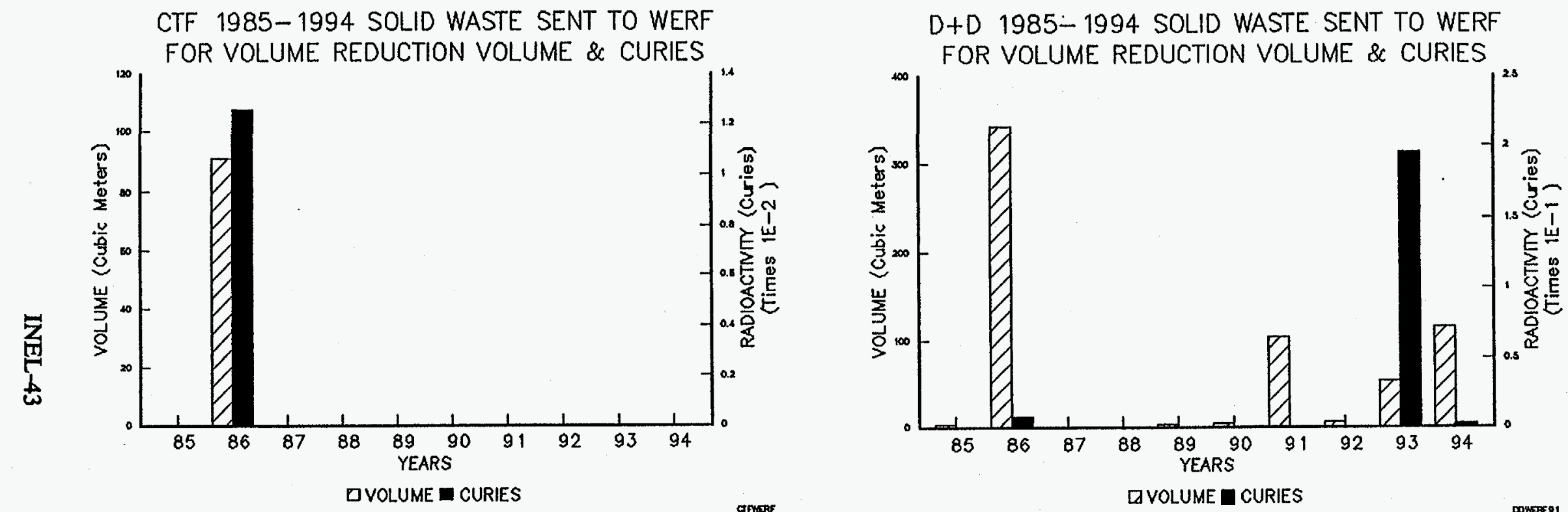

LOF 1985- 1994 SOLID WASTE SENT TO WERF FOR VOLUME REDUCTION VOLUME \& CURIES

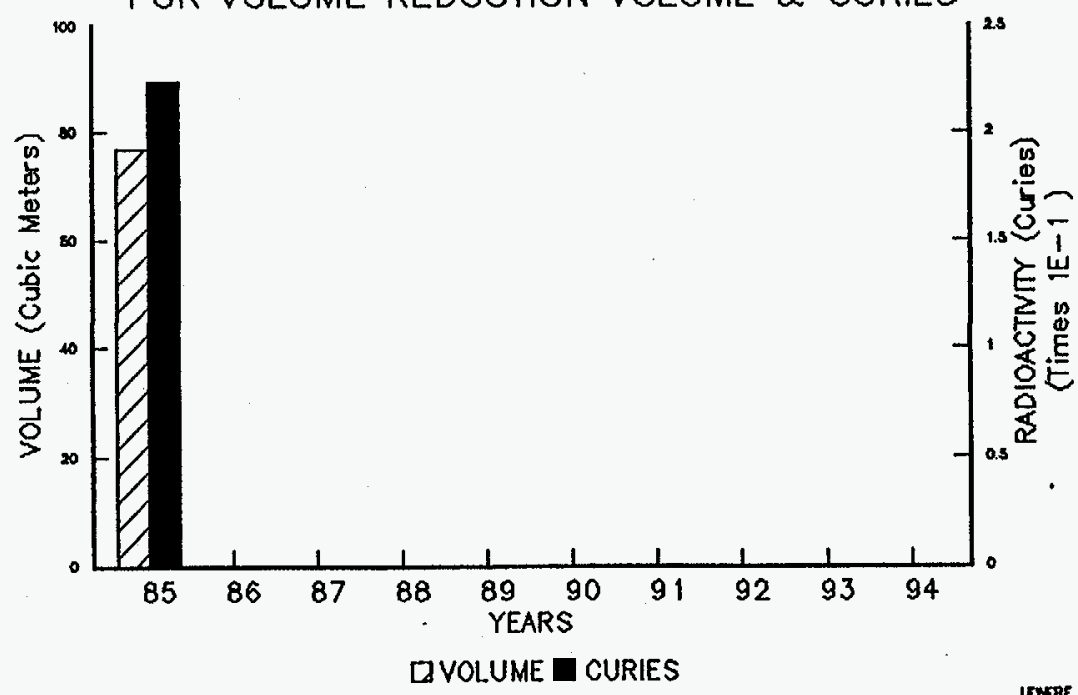

NRF 1985-1994 SOLID WASTE SENT TO WERF FOR VOLUME REDUCTION VOLUME \& CURIES

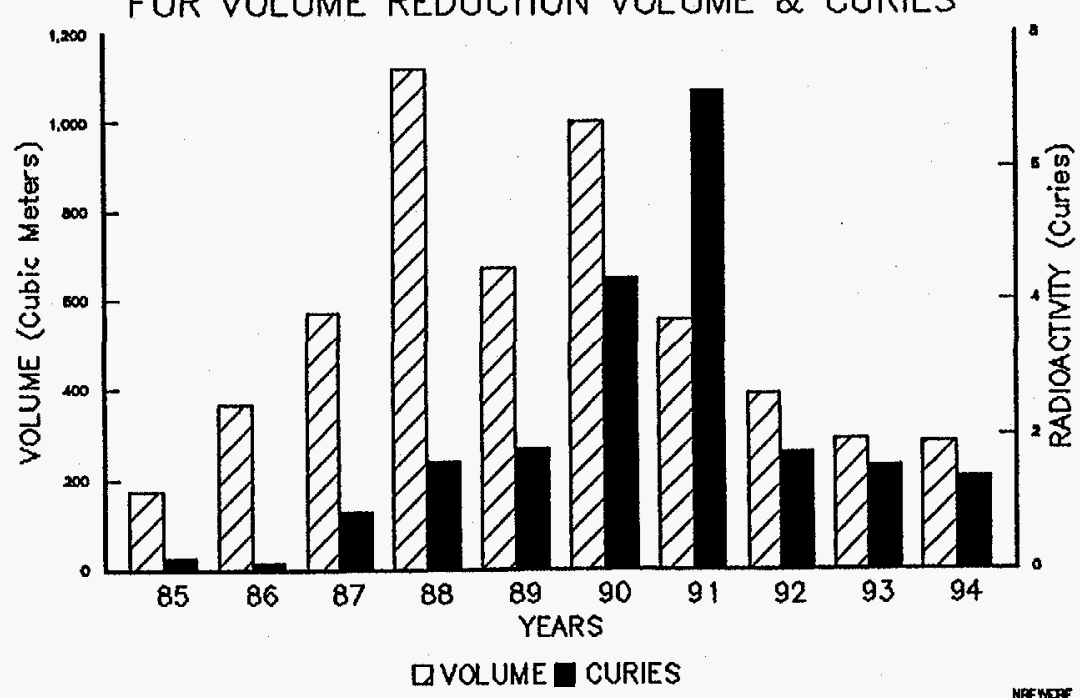




\section{INEL Solid Record-to-Date Summary WERF Waste}
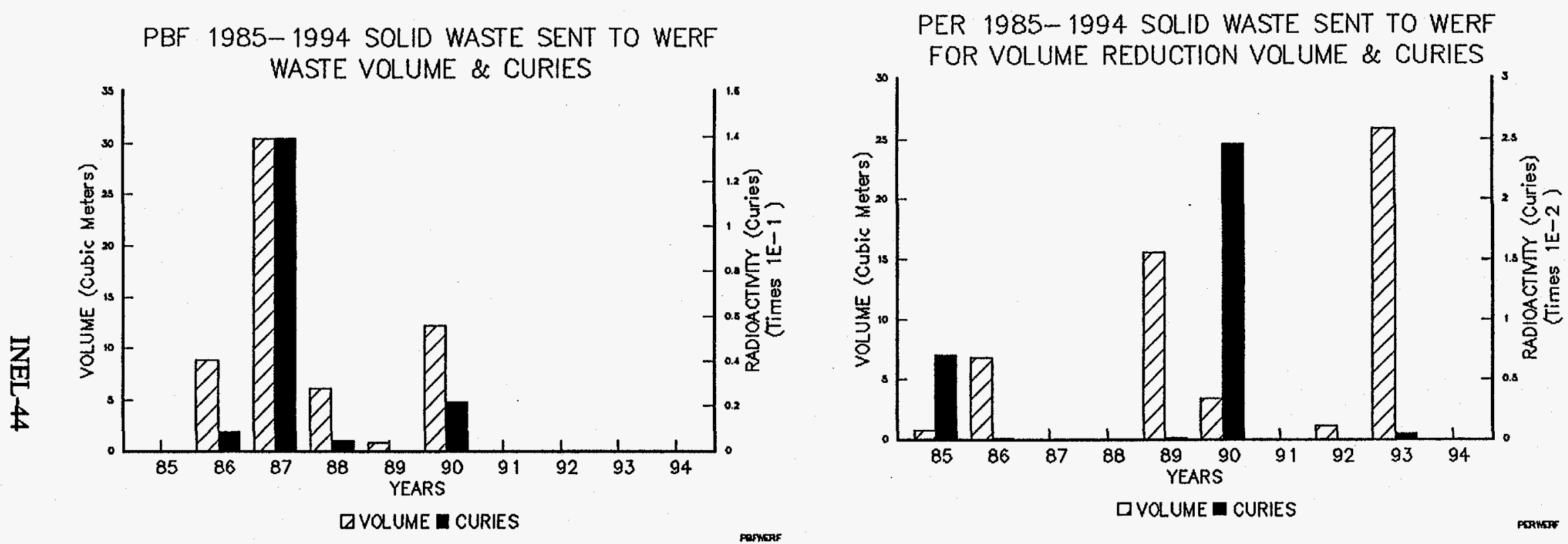

SMC 1985-1994 SOLID WASTE SENT TO WERF

FOR VOLUME REDUCTION VOLUME \& CURIES

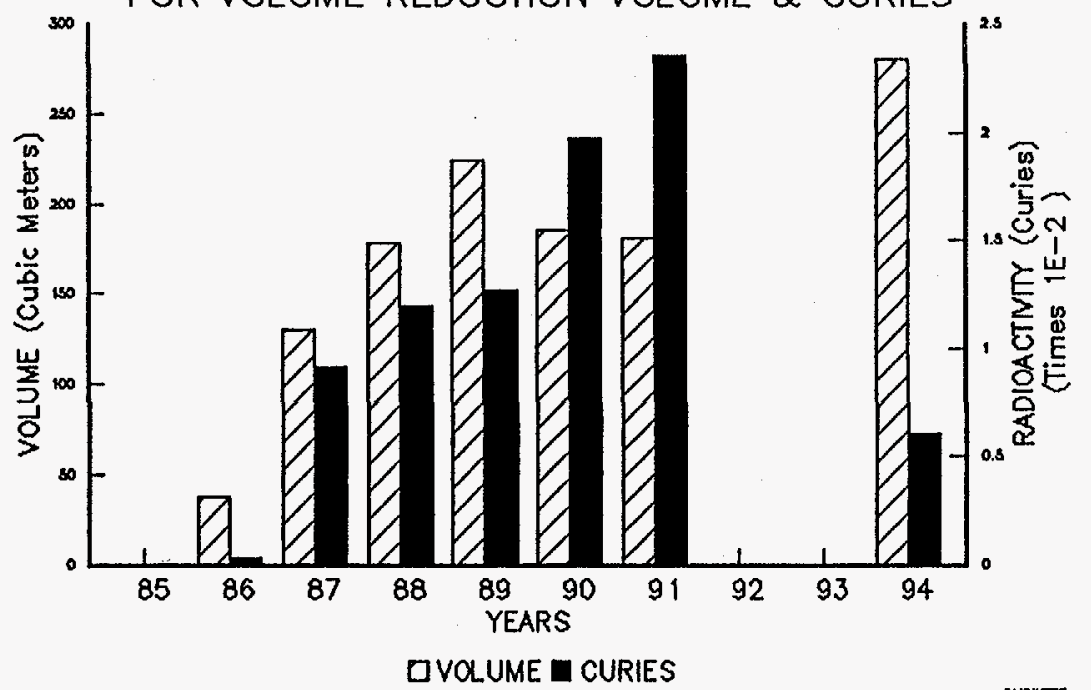




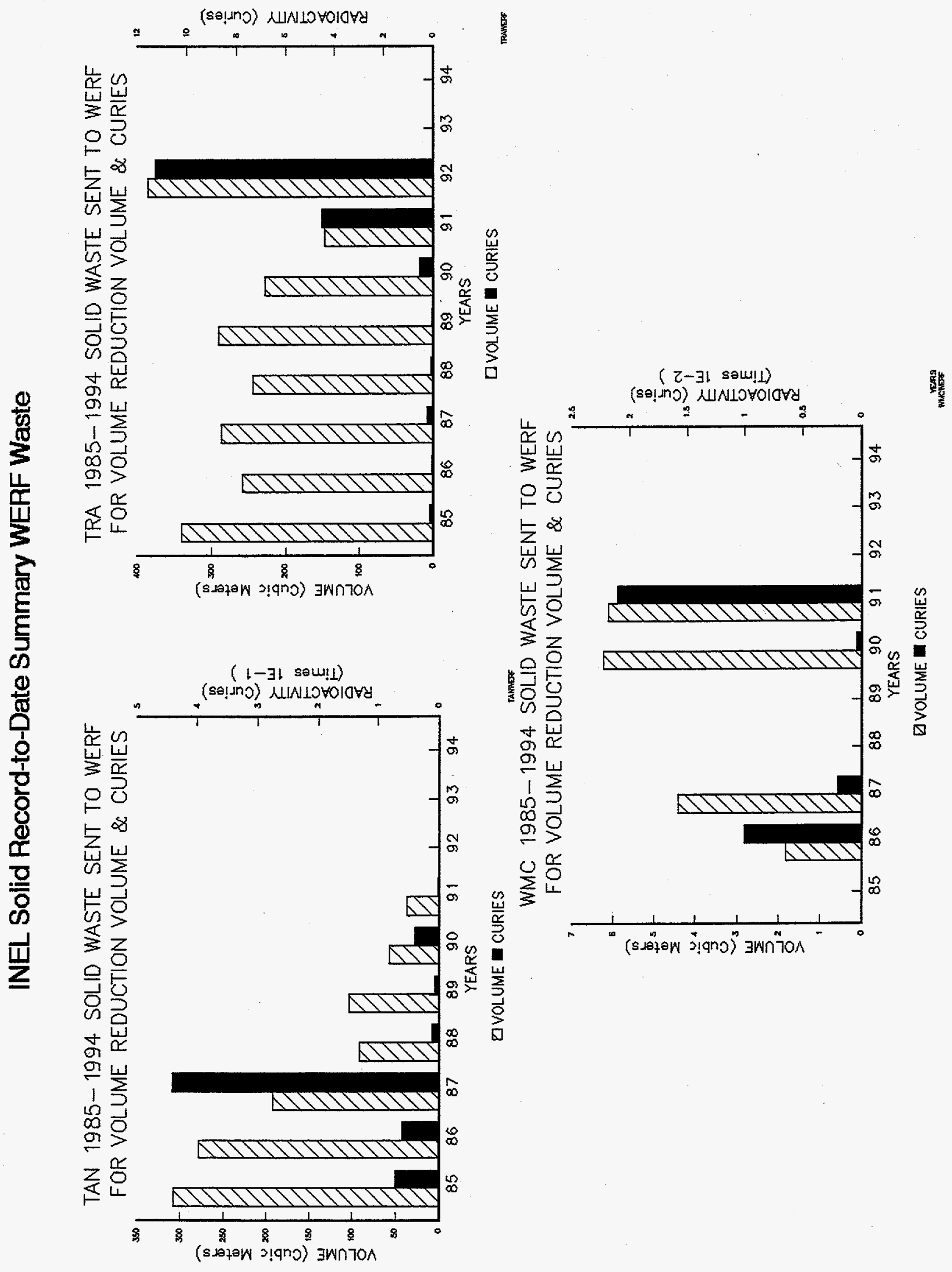


SCND WASTE

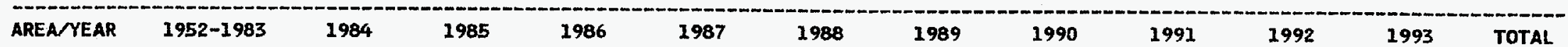

$B+W$

VOLUME

CURIES

$\mathrm{JCH}$

VOLUME

CURIES

MDL

VOLUME

CURTES

案

TOTAL

VOLLATE

CURIES

VOLUME IN CUBIC METERS

\section{4}

46

4

17

491

25

DETAILS MAY NOT ADD UP TO TOTALS BECAUSE OF ROUNDING

THIS IS A SUMMARY OF SCND WASTE NOT PREVIOUSLY REPORTED 


\section{INEL Record-to-Date SCND Summary CY 1994}

$B+W$ 1985- 1994 SPECIAL CASE NON-DEFENSE WASTE STORED AT THE INEL VOLUME \& CURIES

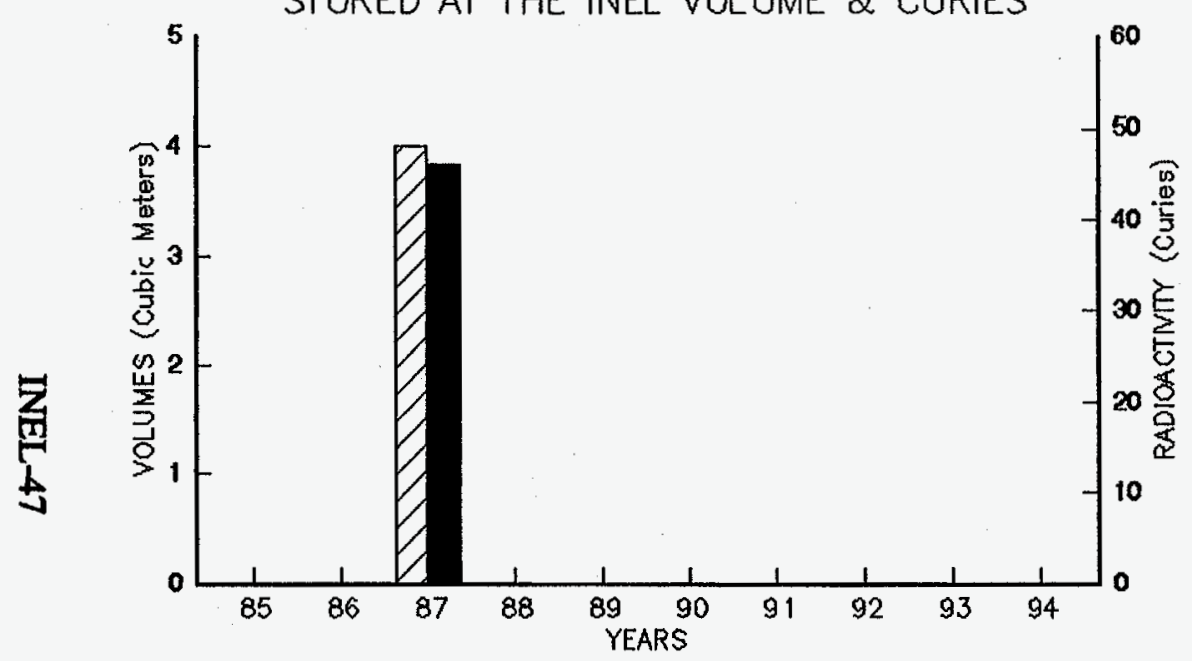

$\square$ VOLUME W CURIES
JCH 1985-1994 SPECIAL CASE NON-DEFENSE WASTE STORED AT THE INEL VOLUME \& CURIES

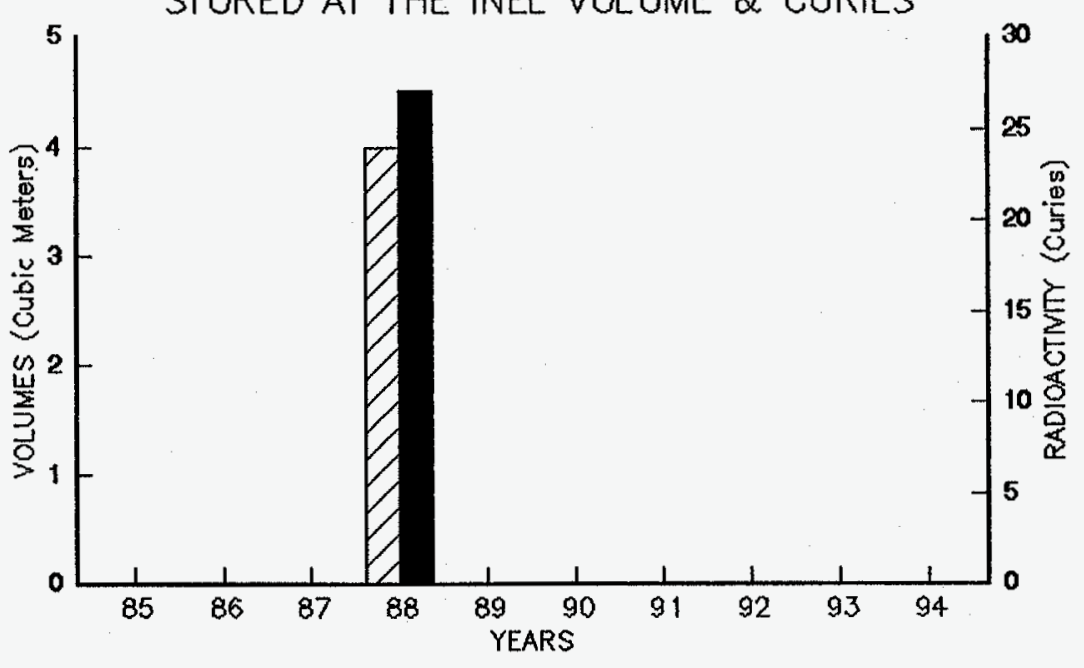

QVOLUME I CURIES

MDL 1985-1994 SPECIAL CASE NON-DEFENSE WASTE

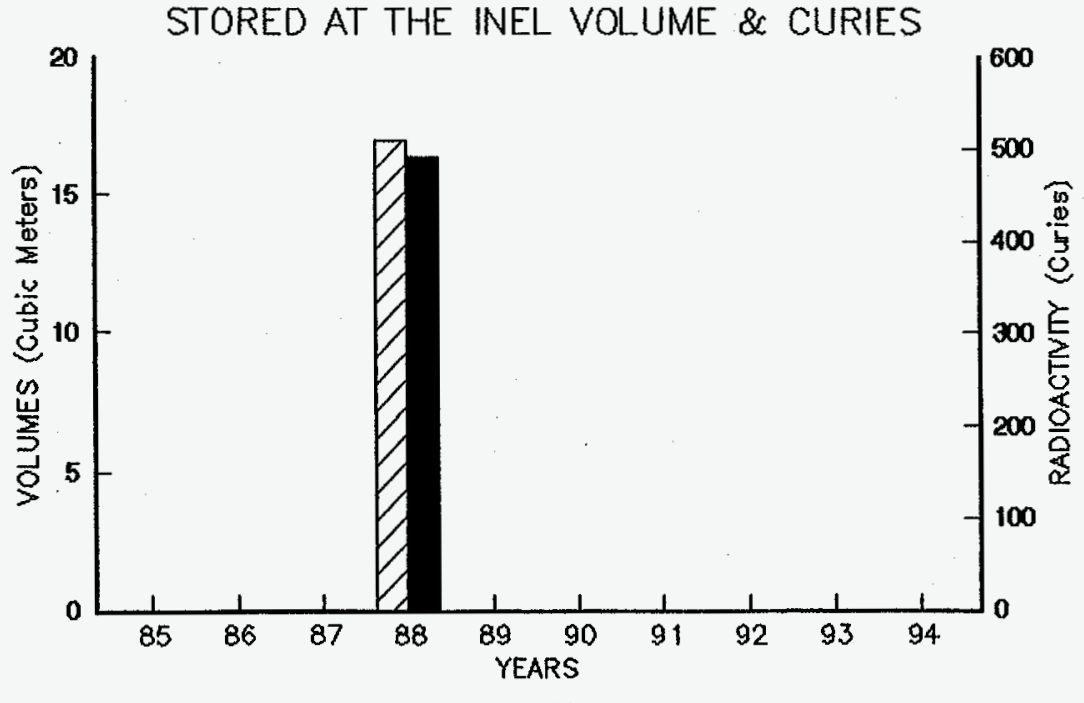

DVOLUME $\square$ CURIES 


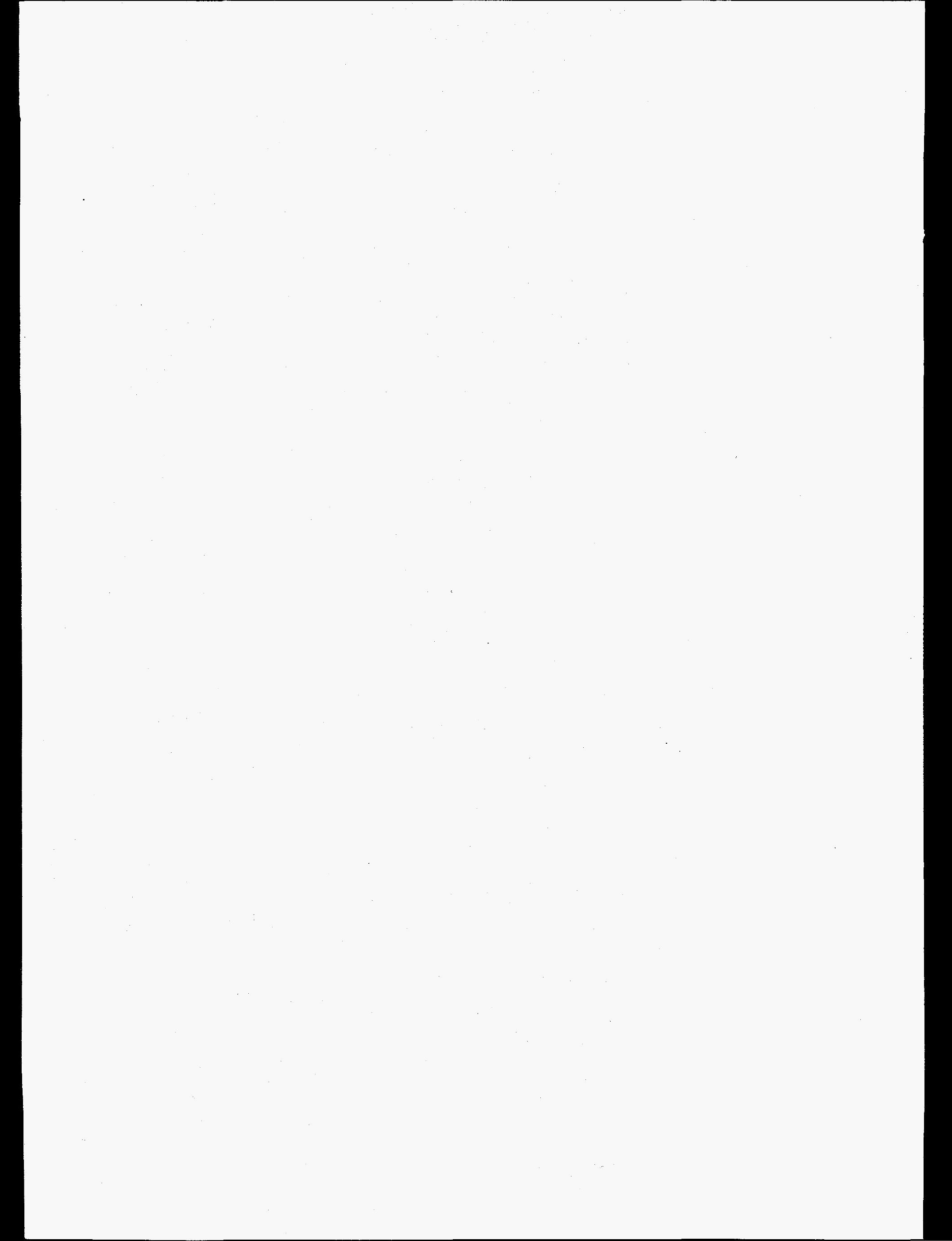


INEL Record-to-Date Decay Summaries

INEL Airborne Waste Decay Data and Bar Graph .............. INEL-51

INEL Liquid Waste Decay Data and Bar Graph ............... INEL-52

INEL Solid Disposed Waste Decay Data and Bar Graph . . . . . . . . . . . INEL-53 

AIRBORNE MASTE DECAY DATA

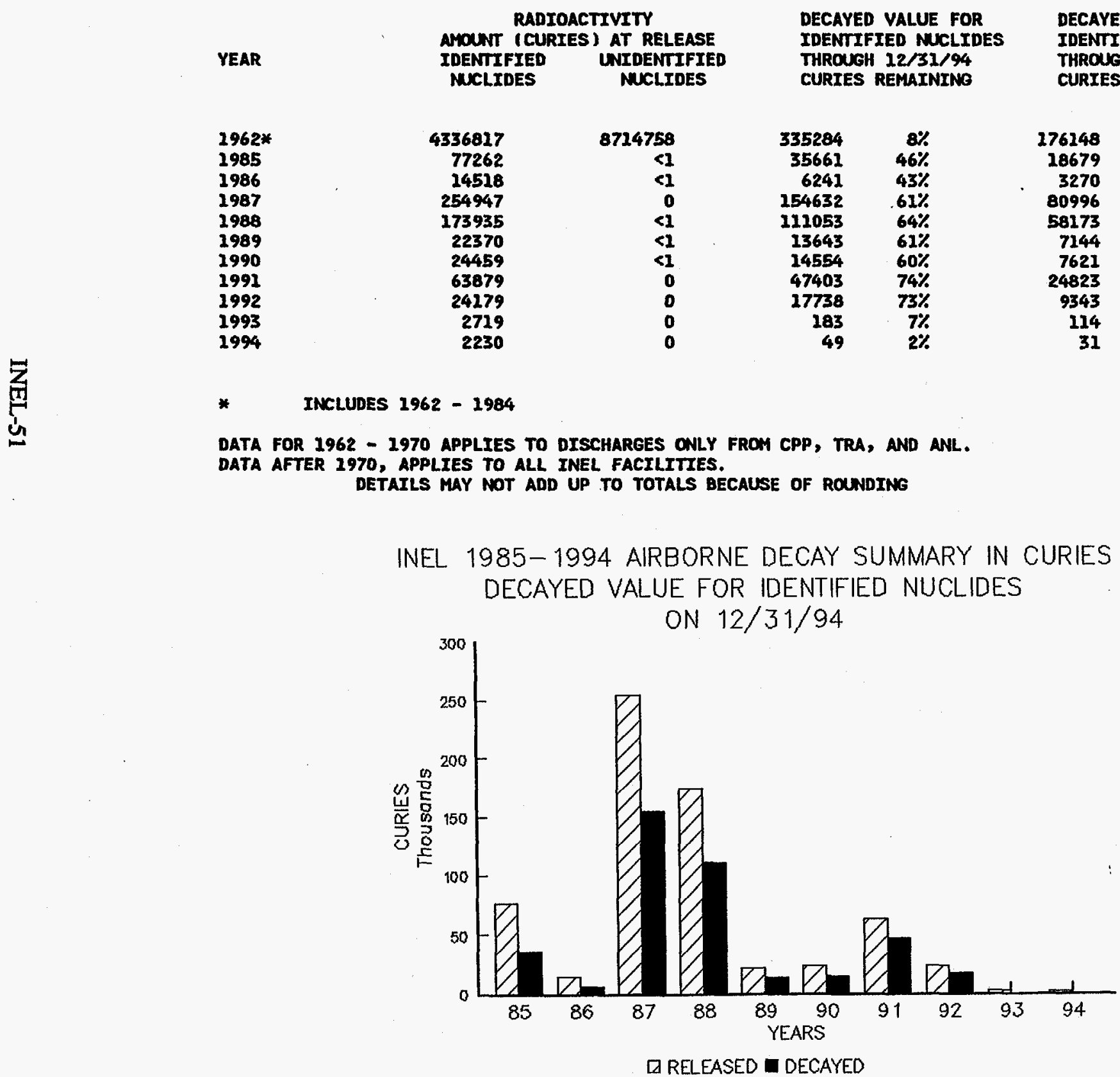


LIGUTO WASTE DECAY DATA

\begin{tabular}{|c|c|c|c|c|c|c|}
\hline YEAR & $\begin{array}{l}\text { AMOUNT ICU } \\
\text { IDENTIFIED } \\
\text { NUCLIDES }\end{array}$ & $\begin{array}{l}\text { AT RELEASE } \\
\text { UNIDENTIFIED } \\
\text { NUCLIDES }\end{array}$ & $\begin{array}{l}\text { IDEN } \\
\text { THRO } \\
\text { CURI }\end{array}$ & $\begin{array}{l}\text { ED NUCLIDES } \\
12 / 31 / 94 \\
\text { EMAINING }\end{array}$ & $\begin{array}{l}\text { IDEN } \\
\text { THRO } \\
\text { CURIE }\end{array}$ & $\begin{array}{l}\text { ED NX } \\
12 / 31 \\
\text { EMAIN. }\end{array}$ \\
\hline $\begin{array}{l}1962 * \\
1985 \\
1986 \\
1987 \\
1988 \\
1989 \\
1990 \\
1991 \\
1992 \\
1993 \\
1994\end{array}$ & $\begin{array}{r}44636 \\
667 \\
346 \\
357 \\
270 \\
137 \\
189 \\
170 \\
187 \\
130 \\
50\end{array}$ & $\begin{array}{r}29310 \\
<1 \\
<1 \\
0 \\
0 \\
0 \\
0 \\
0 \\
0 \\
0 \\
0\end{array}$ & $\begin{array}{r}6024 \\
391 \\
204 \\
227 \\
183 \\
94 \\
143 \\
138 \\
159 \\
115 \\
47\end{array}$ & $\begin{array}{l}13 \% \\
59 \% \\
59 \% \\
63 \% \\
68 \% \\
69 \% \\
76 \% \\
81 \% \\
85 \% \\
88 \% \\
93 \%\end{array}$ & $\begin{array}{r}3465 \\
223 \\
116 \\
129 \\
104 \\
54 \\
82 \\
78 \\
90 \\
65 \\
26\end{array}$ & $\begin{array}{r}8 \% \\
33 \% \\
34 \% \\
36 \% \\
39 \% \\
39 \% \\
43 \% \\
46 \% \\
48 \% \\
50 \% \\
52 \%\end{array}$ \\
\hline
\end{tabular}

* INCLUdES 1962 - 1984

DATA FOR 1962 - 1970 APPLIES TO DISCHARGES ONLY FROM CPP, TRA, AND NRF. DATA AFTER 1970, APPLIES TO ALL INEL FACILITIES.

DETAILS MAY NOT ADO UP TO TOTALS BECAUSE OF ROUNDING

INEL 1985- 1994 LIQUID DECAY SUMMARY

IN CURIES, DECAYED VALUE FOR IDENTIFIED

NUCLIDES ON $12 / 31 / 94$

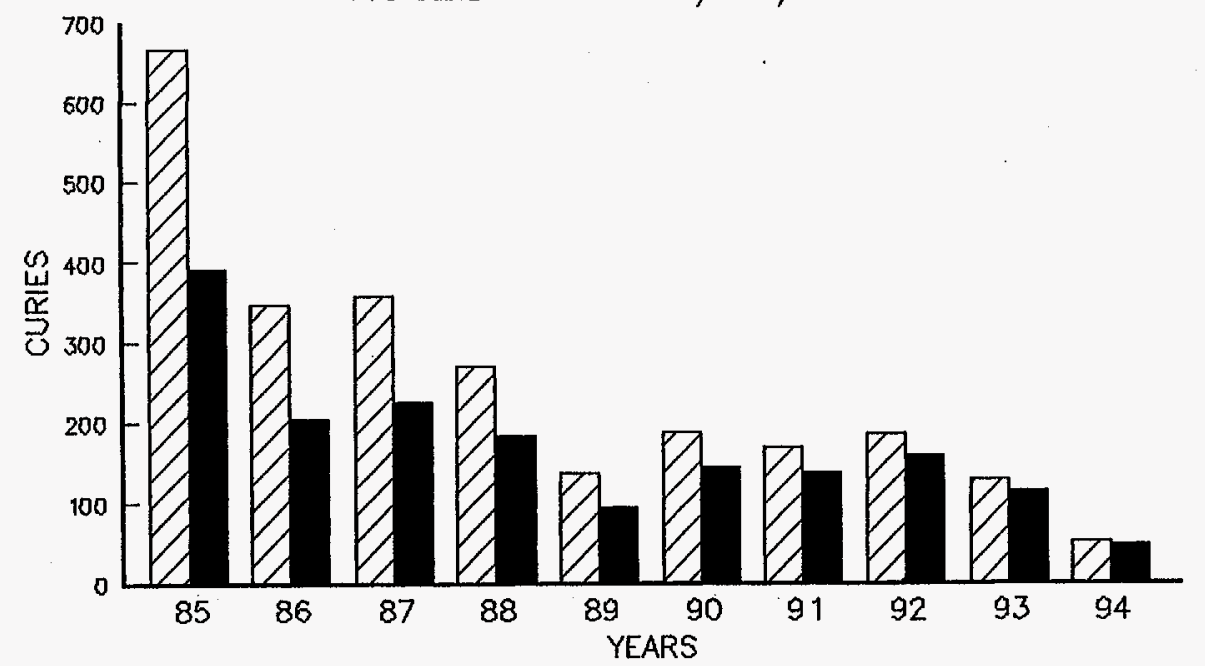

$\square$ RELEASED D DECAYED 
SOLID DISPOSED MASTE DECAY DATA

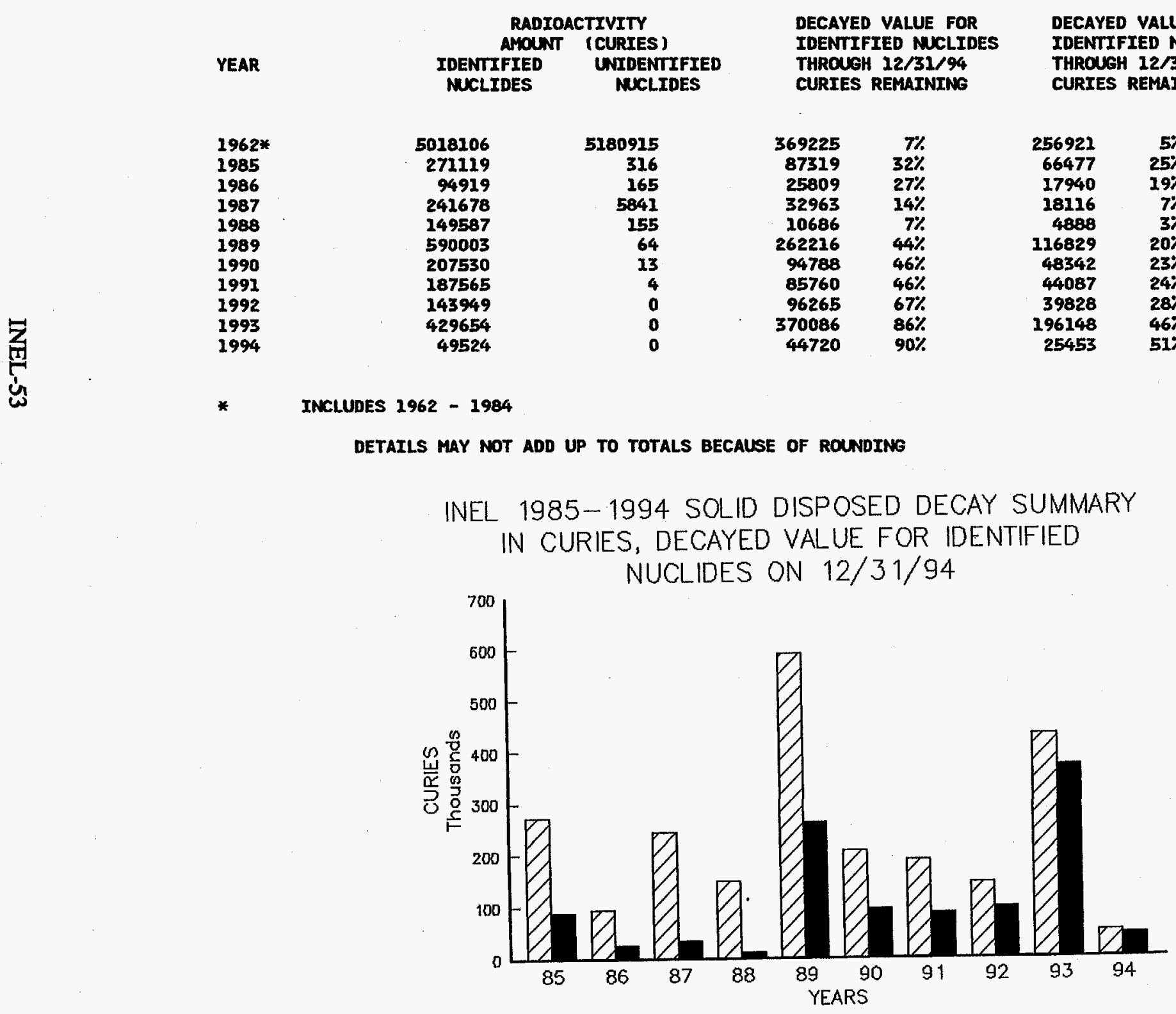

$\square$ DISPOSED DECAYED 


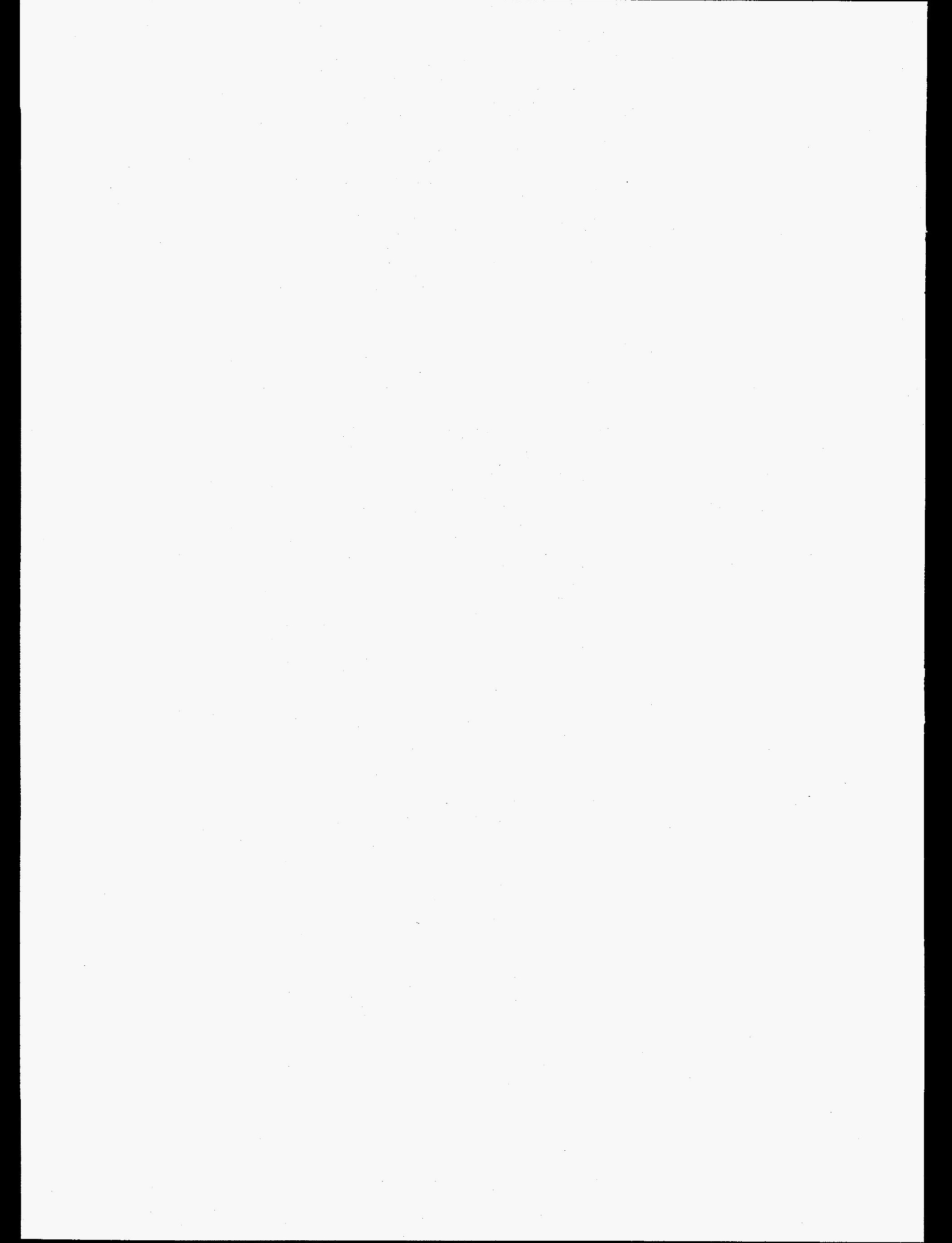




\section{Area Record-to-Date Decay Summaries}

ANL-East Solid Disposed Waste Decay Data and Bar Graph . . . . . . . . . . INEL-57 ANL-West Airborne Effluent Decay Data and Bar Graph . . . . . . . . . . . . ANL-West Liquid Effluent Data

INEL-58

ANL-West Solid Disposed Waste Decay Data and Bar Graph . . . . . . . . . . . ARA Airborne Effluent Decay Data . . . . . . . . . . . . . . . .

ARA Liquid Effluent Data

INEL-59

INEL-60

INEL-61

INEL-62

ARA Solid Disposed Waste Decay Data and Bar Graph . . . . . . . . . . . .

Bendix Solid Disposed Waste Decay Data . . . . . . . . . . . . . . .

BNL Solid Disposed Waste Decay Data . . . . . . . . . . . . . .

CFA Airborne Effluent Decay Data

INEL-63

INEL-64

INEL-65

INEL-66

CFA Liquid Effluent Data and Bar Graph

INEL-67

CFA Solid Disposed Waste Decay Data

INEL-68

CEG Solid Disposed Waste Decay Data

INEL-69

CPP Airborne Effluent Decay Data and Bar Graph . . . . . . . . . . . . . . . INEL-70

CPP Liquid Effluent Data and Bar Graph . . . . . . . . . . . . . . . . . . . INEL-71

CPP Solid Disposed Waste Decay Data and Bar Graph . . . . . . . . . . . . . INEL-72

CTF Airborne Effluent Decay Data . . . . . . . . . . . . . . . . . . . . INEL-73

CTF Liquid Effluent Data . . . . . . . . . . . . . . . . . . . . . . . INEL-74

CTF Solid Disposed Waste Decay Data and Bar Graph . . . . . . . . . . . . . INEL-75

D\&D Solid Disposed Waste Decay Data and Bar Graph . . . . . . . . . . . . . . . INEL-76

LOFT Facility Airborne Effluent Decay Data and Bar Graph . . . . . . . . . . . . INEL-77

LOFT Facility Liquid Effluent Decay Data . . . . . . . . . . . . . . . . . . . . . INEL-78

LOFT Facility Solid Disposed Waste Decay Data . . . . . . . . . . . . . . . INEL-79

NRF Airborne Effluent Decay Data . . . . . . . . . . . . . . . . . . . INEL-80

NRF Liquid Effluent Data . . . . . . . . . . . . . . . . . . . . . . . . INEL-81

NRF Solid Disposed Waste Decay Data and Bar Graph . . . . . . . . . . . . . . INEL-82

PBF Airborne Effluent Decay Data . . . . . . . . . . . . . . . . . INEL-83

PBF Liquid Effluent Data . . . . . . . . . . . . . . . . . . . . . . . . INEL-84

PBF Solid Disposed Waste Decay Data and Bar Graph . . . . . . . . . . . . INEL-85

RFO Solid Disposed Waste Decay Data . . . . . . . . . . . . . . . . . . INEL-86

SMC Airborne Effluent Decay Data . . . . . . . . . . . . . . . . . . INEL-87

SMC Solid Disposed Waste Decay Data . . . . . . . . . . . . . . . . . INEL-88

SPERT Airborne Effluent Decay Data . . . . . . . . . . . . . . . . . . . . INEL-89

SPERT Liquid Effluent Data . . . . . . . . . . . . . . . . . . . . . . . INEL-90

SPERT Solid Disposed Waste Decay Data . . . . . . . . . . . . . . . . . . INEL-91

TAN Airborne Effluent Decay Data . . . . . . . . . . . . . . . . . . . . . INEL-92

TAN Liquid Effluent Data ... . . . . . . . . . . . . . . . . . . . . . . . INEL-93

TAN Solid Disposed Waste Decay Data and Bar Graph . . . . . . . . . . . . . . . INEL-94

TRA Airborne Effluent Decay Data and Bar Graph . . . . . . . . . . . . . . . . INEL-95

TRA Liquid Effluent Data and Bar Graph . . . . . . . . . . . . . . . . . . . INEL-96

TRA Solid Disposed Waste Decay Data and Bar Graph . . . . . . . . . . . . . . INEL-97

WERF Airborne Effluent Decay Data . . . . . . . . . . . . . . . . . . . INEL-98

WERF Solid Disposed Waste Decay Data . . . . . . . . . . . . . . . . . . . INEL-99

WMC Airborne Effluent Decay Data . . . . . . . . . . . . . . . . . . . INEL-100

WMC Liquid Effluent Data . . . . . . . . . . . . . . . . . . . . . . INEL-101

WMC Solid Disposed Waste Decay Data and Bar Graph . . . . . . . . . . . . INEL-102 

SOLID DISPOSED MASTE DECAY DATA

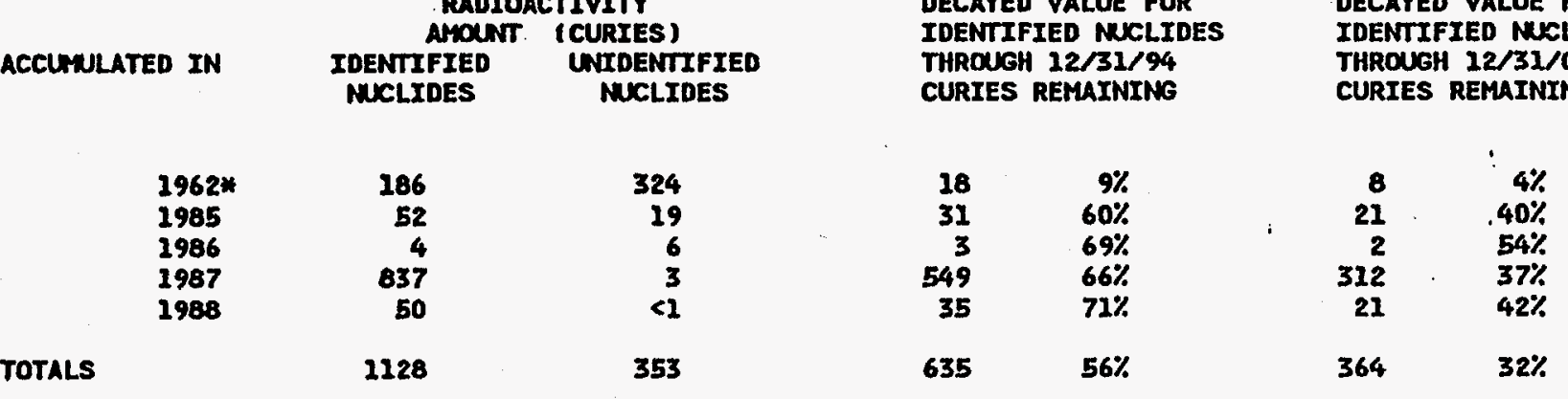

* INCLUDES $1962-1984$

DETAILS MAY NOT ADD UP TO TOTALS BECAUSE OF ROUNDING

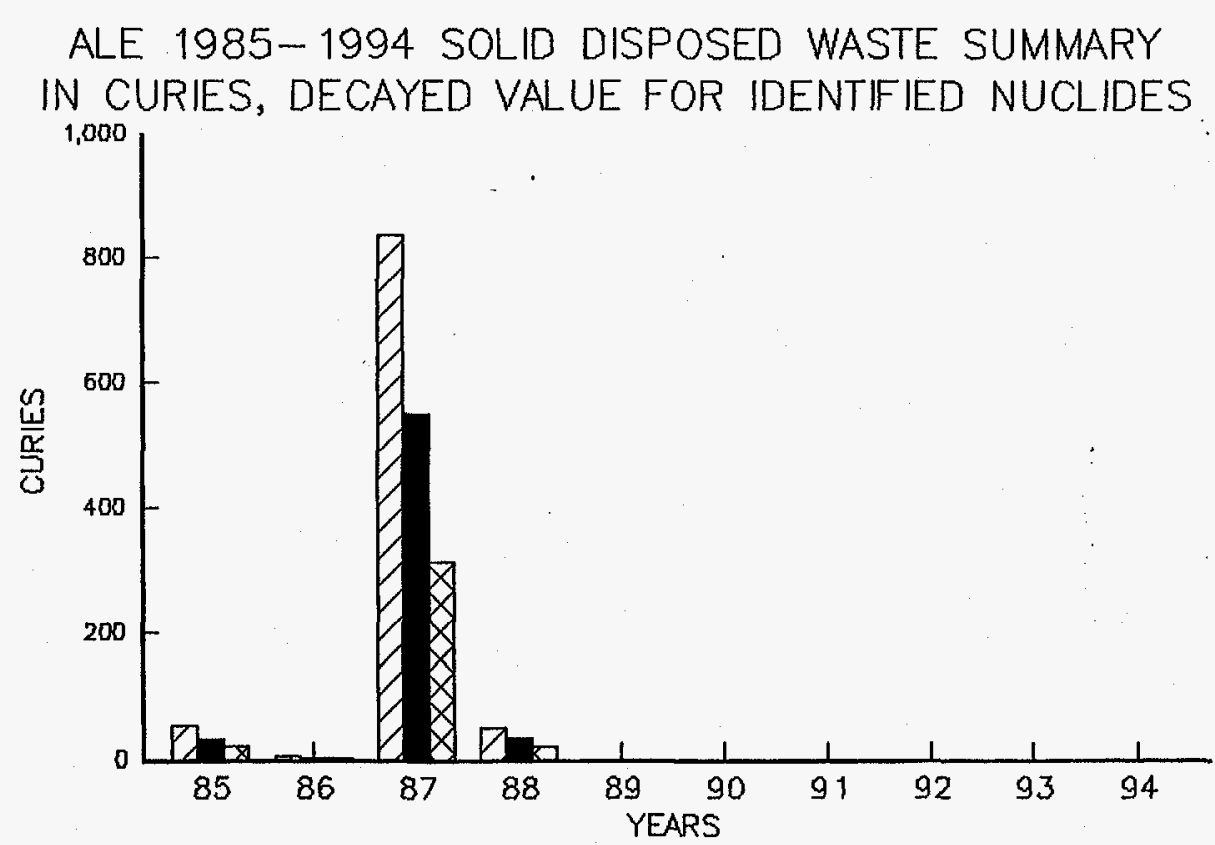

口DISPOSED DECAYED ON 12/31/94 D DECAYED ON $12 / 31 / 04$ 
ATRBORNE EFFLUENT DECAY DATA

RACIOACTIVITY

AMOUNT (CURTES) AT RELEASE

IDENTIFIED UNIDENTIFIED

NUCLIDES

MUCLIDES

2962* 32865

1985

1986

1987

1988

1989

1990

1991

1992

1993

1994

TOTALS

32865
916
932
889
618
686
716
551
739
1160
1154

41227

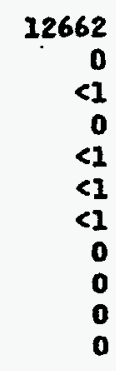

12662
DECAYED VALUE FOR

IDENTIFIED MUCLIDES

THROUGH 12/31/94

CURIES REMAINING

$\begin{array}{rr}617 & 2 \% \\ 5 & <1 \% \\ 5 & <1 \% \\ 6 & <1 \% \\ 2 & <1 \% \\ 1 & <1 \% \\ 2 & <1 \% \\ 1 & <1 \% \\ 167 & 23 \% \\ 125 & 11 \% \\ 46 & 4 \% \\ 977 & 2 \%\end{array}$

DECAYED VALUE FOR

IDENIIFIED MUCLIDES

THROUGH 12/31/0

CURIES REMAINING

INCLUDES 1962 - 1984

DETAILS MAY NOT ADD UP TO TOTALS BECAUSE OF ROUNDING

ANL 1985-1994 AIRBORNE DECAY SUMMARY

IN CURIES, DECAYED VALUE FOR IDENTIFIED NUCLIDES

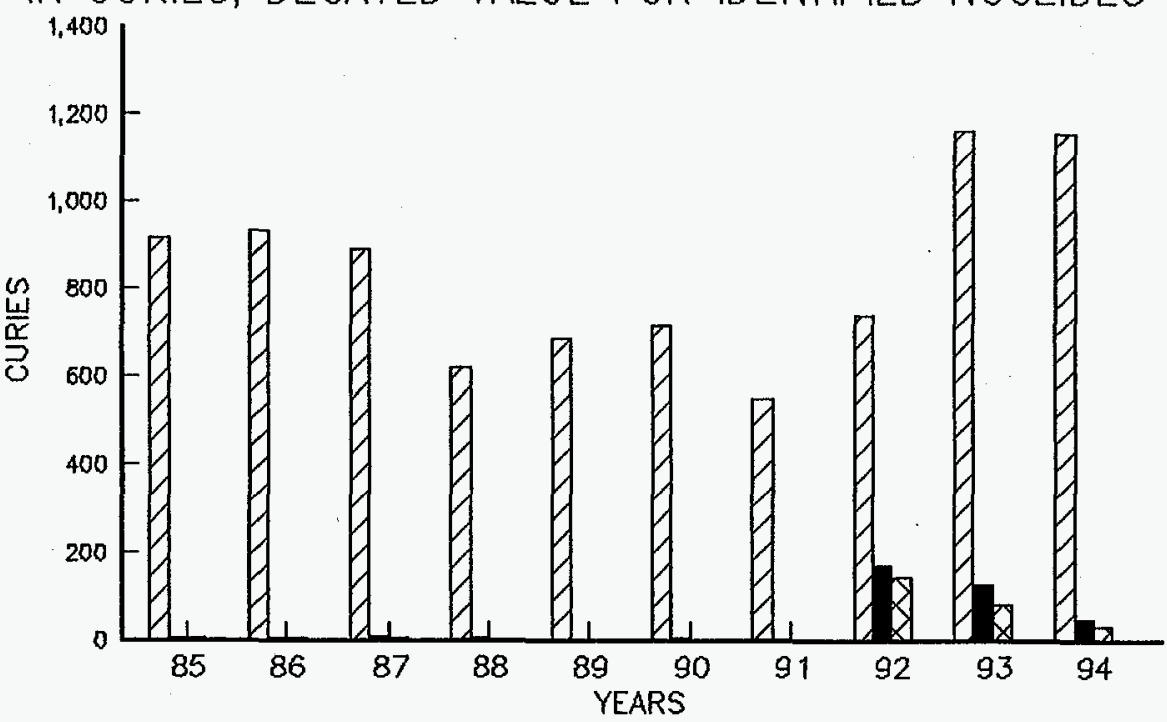

$\square$ RELEASED $\square$ DECAYED ON 12/31/94 $\triangle$ DECAYED ON 12/31/O4 
LIQUID EFFLUENT DATA

RADIOACTIVITY

AMOUNT (CURIES) AT PELEASE

IDENTIFIED UNIDENTIFIED

MUCLIDES

$\begin{array}{ll}1962 * & 2 \\ 1985 & <1 \\ 1986 & <1 \\ 1987 & <1 \\ 1988 & <1 \\ 1989 & <1 \\ 1990 & <1 \\ 1991 & <1 \\ 1992 & <1 \\ 1993 & <1 \\ 1994 & <1\end{array}$

TOTALS

IKCLUDES $1962-1984$

DETAILS MAY NOT ADD UP TO TOTALS BECAUSE OF ROUNDING

DECAYEd VALUE FOR $\begin{array}{ll}\text { TDENTIFIED MUCLIDES } & \text { IDENTIFIED MUCLIDES } \\ \text { THROUGH 12/31/94 } & \text { THROUGH 12/31/04 }\end{array}$ $\begin{array}{ll}\text { TDENTIFIED NUCLIDES } & \text { IDENTIFIED NUCLID } \\ \text { THROUGH 12/31/94 } & \text { THROUGH 12/31/04 }\end{array}$ THROUGH 12/31/94 CURIES REMAINING

$\begin{array}{ll}<1 & 27 \% \\ <1 & 34 \% \\ <1 & 35 \% \\ <1 & 37 \% \\ <1 & 40 \% \\ <1 & 41 \% \\ <1 & 44 \% \\ <1 & 47 \% \\ <1 & 50 \% \\ <1 & 53 \% \\ <1 & 55 \%\end{array}$

1

$34 \%$ 
SOLID DISPOSED WASTE DECAY DATA

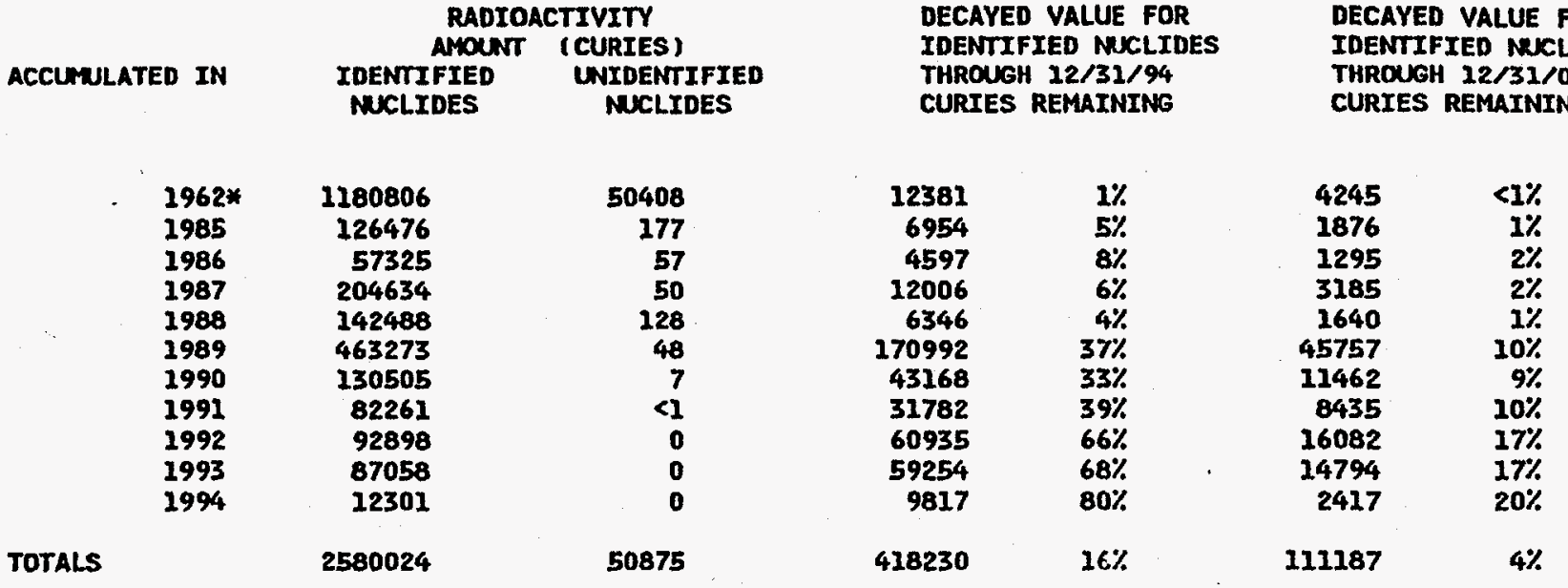

DETAILS MAY NOT ADD UP TO TOTALS BECALSE OF ROUNDING

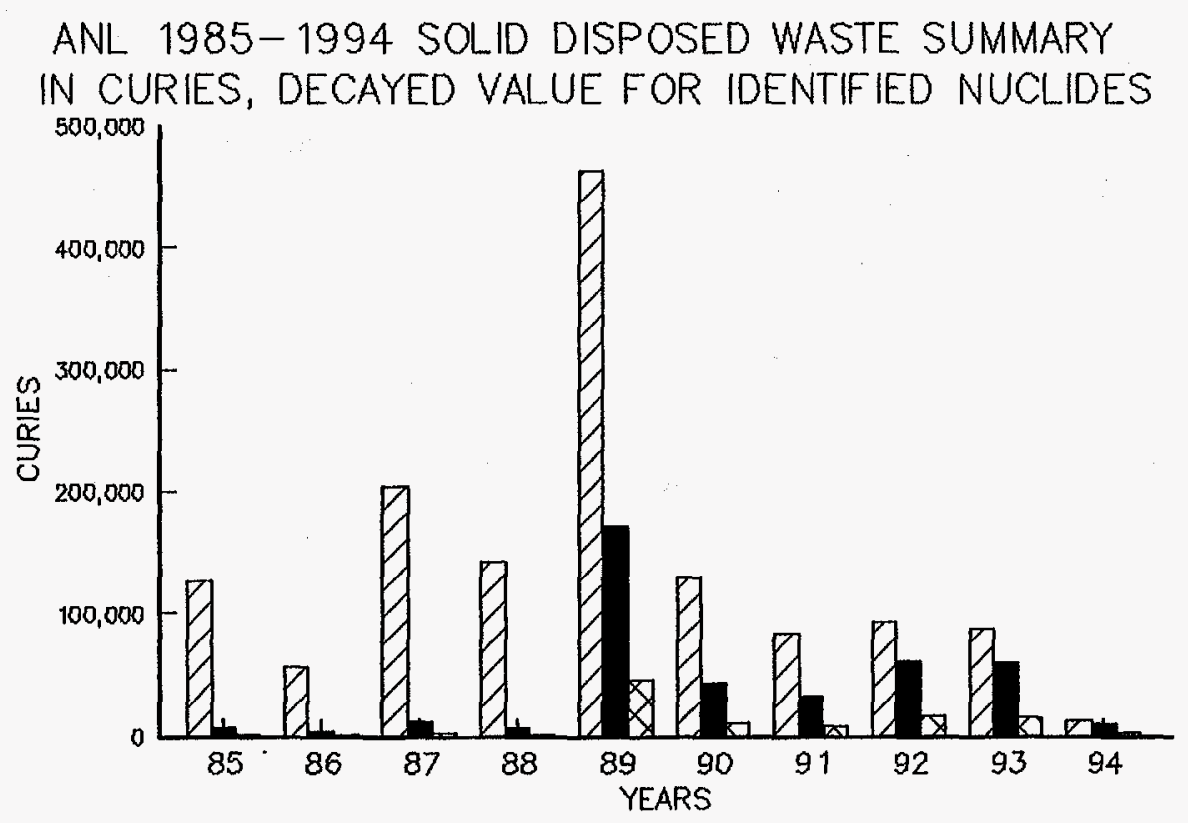

IDISPOSED DECAYED ON $12 / 31 / 94 \square D E C A Y E D$ ON $12 / 31 / 04$ 
AIRBORNE EFFLUENT DECAY DATA

RADIOACTIVITY

ACCUNULATED IN

$$
\begin{aligned}
& \text { RADIOACTIVITY } \\
& \text { AMOUNT (CURIES) AT RELEASE } \\
& \text { IDENTIFIEN } \\
& \text { NUCLIDES }
\end{aligned}
$$

DECAYED VALUE FOR IDENTIFIED NKCLIDES

THROUGH 12/3.1/94

CURIES REMAINING

$\begin{array}{ll}1962 * & <1 \\ 1985 & <1 \\ 1986 & <1 \\ 1987 & <1 \\ 1988 & <1\end{array}$

TOTALS

$\begin{array}{lr}<1 & <1 \\ <1 & <1 \\ <1 & <1 \\ <1 & 0 \\ <1 & 0 \\ <1 & <1\end{array}$

* InCLUDES $1962-1984$

$\begin{array}{rr}<1 & <1 \% \\ <1 & 4 \% \\ <1 & 64 \% \\ <1 & 1 \% \\ <1 & 3 \% \\ <1 & <1 \%\end{array}$

$<1<1 \%$
DECAYEd VALUE FOR IDENTIFIED MUCLIDES THROUGH 12/31/04 CURIES REMAINING

DETAILS MaY NOT ADD UP TO TOTALS BECAUSE OF ROUNDING 
IIQUID EFFLUENT DATA

\section{RADIOACTIVITY \\ AMOUNT (CURIES) AT RELEASE IDENTIFIED \\ WIDENTIFIED}

ACCUNULATED IN

$1962 *$
1986
1987
1988

TOTALS

*

INCLUDES $1962-1984$

$<1$

$<1$

$<1$

I
0
0

\section{DECAYEd VALUE FOR \\ IDENTIFIED MUCLIDES \\ THROUEH 12/31/94 \\ CURIES REMAINING}

DETAILS MAY NOT ADD UP TO TOTALS BECAUSE OF ROUNDING
$0 \%$

$0 \quad 0 \%$

$0.0 \%$

$<1<1 \%$
DECAYED VALUE FOR

IDENTIFIED MCLIDES

THROUGH 12/31/04

CURIES REMAINING

$\begin{array}{rr}0 & 0 \% \\ <1 & 9 \% \\ 0 & 0 \% \\ 0 & 0 \% \\ <1 & <1 \%\end{array}$

$0 \%$
$1 \%$ (1) 
SOLID DISPOSED WASTE DECAY DATA

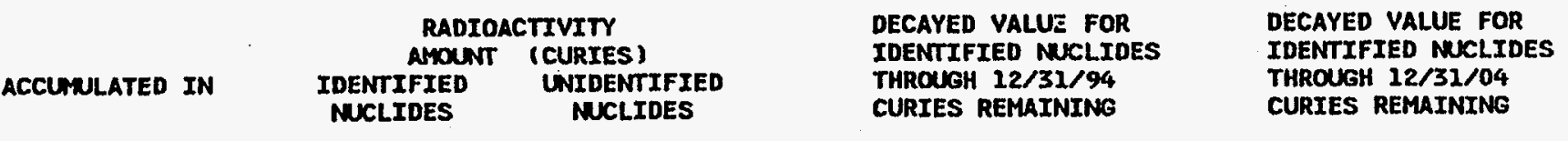

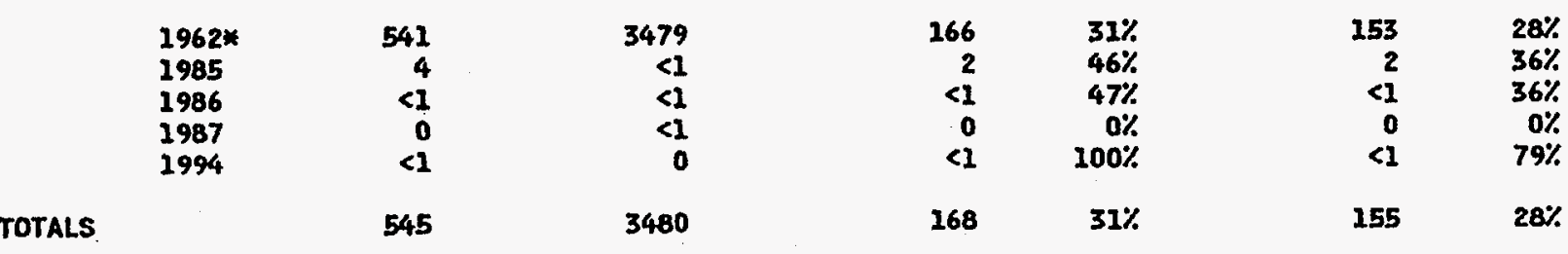

* INCLUDES 1962 - 1984

DETAILS MAY NOT ADD UP TO TOTALS BECAUSE OF ROUNDING

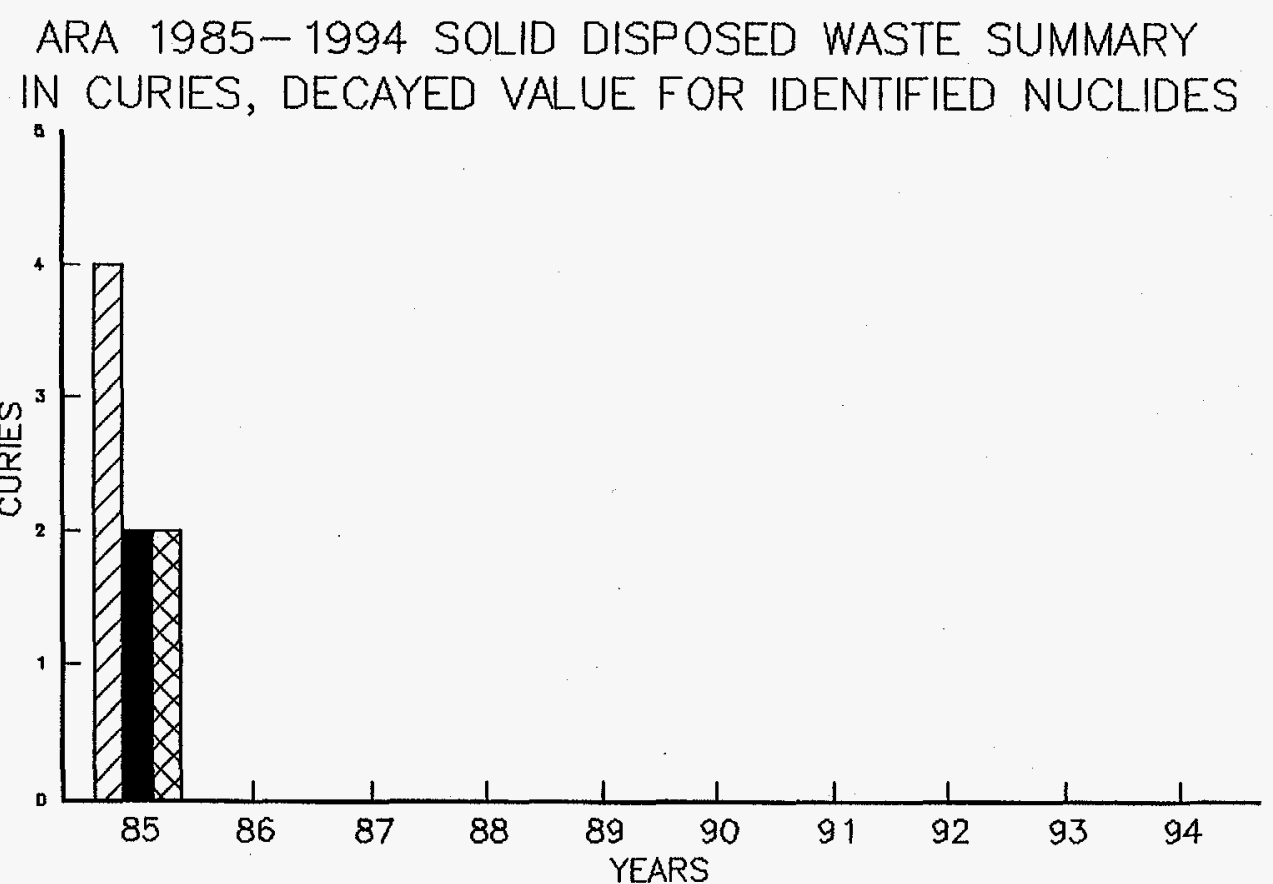

$\square$ DISPOSED $\square$ DECAYED ON $12 / 31 / 94$ Q DECAYED ON $12 / 31 / 04$ 


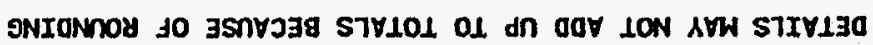

789

$\% 89$

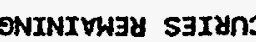

†O/TE/ZI HตnOUH।

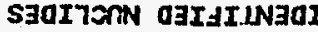

yos zก7ษ azAษว30

T>

Is
766

$\% 62$

โ>

SNINIษW9y saI4nว

\$6/TE/ZT HONO४4H

SzaI7FN 03IATIN30I

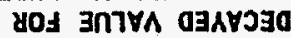

\$96I - 296I S3an73NI

T>

T) $986 \mathrm{~T}$

VIVO AFכ30 JLSFM OJSOdSIa oI7OS 


\begin{tabular}{|c|c|c|c|c|c|c|}
\hline ACCUMLATED IN & $\begin{array}{l}\text { RADIC } \\
\text { AHOUNI } \\
\text { IDENTIFIED } \\
\text { NUCLIDES }\end{array}$ & $\begin{array}{l}\text { TIVITY } \\
\text { (CURIES) } \\
\text { UNIDENTIFIED } \\
\text { MUCLIDES }\end{array}$ & \multicolumn{2}{|c|}{$\begin{array}{l}\text { DECAYED VALUE FOR } \\
\text { IDENTIFIED NHCLIIDES } \\
\text { THROUGH } 12 / 31 / 94 \\
\text { CURIES REMAINING }\end{array}$} & \multicolumn{2}{|c|}{$\begin{array}{l}\text { DECAYED VALUE } \\
\text { IDENTIFIED NUE } \\
\text { THROUGH } 12 / 31 / \\
\text { CURIES REMAINI }\end{array}$} \\
\hline 1962* & $<1$ & 0 & $<1$ & $51 \%$ & $<1$ & $29 \%$ \\
\hline TOTALS & $<1$ & 0 & $<1$ & $51 \%$ & $<1$ & $29 \%$ \\
\hline
\end{tabular}

* INClUdes $1962-1984$

DETAILS MAY NOT ADD UP TO TOTALS BECAUSE OF ROUNDING 
TOTALS

1962*

1985

1986

1987

1988

1989

1990

1991

1992

1993

INCLUDES 1962 - 1984

AIRBORNE EFFLUENT DECAY DATA

\section{RADIOACTIVITY}

AMOUNT (CURIES) AT RELEASE IDENTIFIED UNIDENTIFIED

NUCLIOES
DECAYED VALUE FOR
IDENTIFIED MUCLIDES
THROUEH 12/31/94
CURTES REMATNING

$\begin{array}{rr}<1 & 33 \% \\ <1 & 39 \% \\ <1 & 27 \% \\ <1 & 9 \% \\ <1 & 16 \% \\ <1 & 18 \% \\ <1 & 15 \% \\ <1 & 13 \% \\ <1 & 5 \% \\ <1 & 2 \%\end{array}$

$<1 \quad 19 \%$

$$
\begin{aligned}
& <1 \\
& <1 \\
& <1 \\
& <1 \\
& <1 \\
& <1 \\
& <1 \\
& <1 \\
& <1 \\
& <1
\end{aligned}
$$

$<1$

$<1$

$<1$
$<1$
$<1$
0
0
0
0
0
0
0

DECAYED VALUE FOR

IDENTIFIED NUCLIDES

THROUGH 12/31/04

CURIES REMAINING

$\begin{array}{rr}<1 & 21 \% \\ <1 & 22 \% \\ <1 & 17 \% \\ <1 & 4 \% \\ <1 & 7 \% \\ <1 & 7 \% \\ <1 & 6 \% \\ <1 & 7 \% \\ <1 & 2 \% \\ <1 & 2 \%\end{array}$

DETAILS MAY NOT ADD UP TO TOTALS BECAUSE OF ROUNDING 
LIQUID EFFLUENT DATA

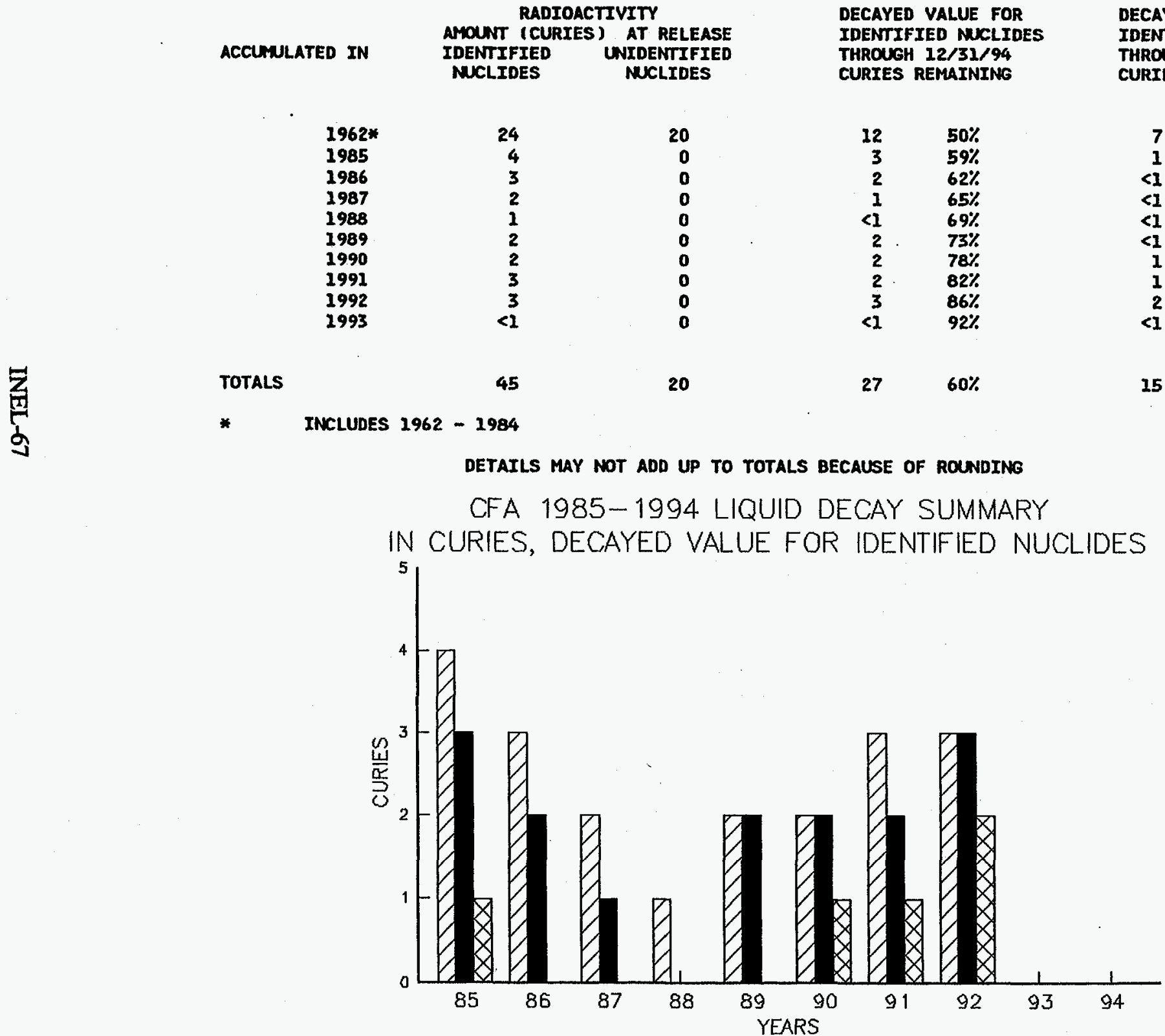

$\square D$ DISPOSED DECAYED ON 12/31/91 $\square$ DECAYED ON 12/31/01 
SOLID DISPOSED MASTE DECAY DATA

\begin{tabular}{|c|c|c|c|c|c|c|}
\hline ACCUMLATED IN & $\begin{array}{l}\text { RADI } \\
\text { AMOUN } \\
\text { IDENTIFIED } \\
\text { MUCLIDES }\end{array}$ & $\begin{array}{l}\text { STVITY } \\
\text { (CURIES) } \\
\text { UNIDENTIFIED } \\
\text { NUCLIOES }\end{array}$ & \multicolumn{2}{|c|}{$\begin{array}{l}\text { DECAYED VALUE FOR } \\
\text { IDENTIFIED MUCLIDES } \\
\text { THROUGH } 12 / 31 / 94 \\
\text { CURTES REMAINING }\end{array}$} & \multicolumn{2}{|c|}{$\begin{array}{l}\text { DECAYED VALUE F } \\
\text { IDENTIFIED NUCL } \\
\text { THROUGH } 12 / 31 / 0 \\
\text { CURIES REMAININ }\end{array}$} \\
\hline $\begin{array}{l}2962 * \\
1985 \\
1986 \\
1987 \\
1988 \\
1989 \\
1990 \\
1993 \\
1994\end{array}$ & $\begin{array}{r}186 \\
<1 \\
0 \\
0 \\
0 \\
0 \\
<1 \\
<1 \\
<1\end{array}$ & $\begin{array}{r}196 \\
<1 \\
<1 \\
4 \\
<1 \\
<1 \\
0 \\
0 \\
0\end{array}$ & $\begin{array}{r}36 \\
<1 \\
0 \\
0 \\
0 \\
0 \\
<1 \\
<1 \\
<1\end{array}$ & $\begin{array}{r}20 \% \\
99 \% \\
0 \% \\
0 \% \\
0 \% \\
0 \% \\
71 \% \\
93 \% \\
98 \%\end{array}$ & $\begin{array}{r}17 \\
<1 \\
0 \\
0 \\
0 \\
0 \\
<1 \\
<1 \\
<1\end{array}$ & $\begin{array}{r}9 \% \\
98 \% \\
0 \% \\
0 \% \\
0 \% \\
0 \% \\
38 \% \\
63 \% \\
74 \%\end{array}$ \\
\hline TOTALS & 186 & 200 & 37 & $20 \%$ & 17 & $9 \%$ \\
\hline
\end{tabular}

* INCLUDES $2962-1984$

DETAILS MAY NOT ADD UP TO TOTALS BECAUSE OF ROUNDING 
CONBUSTIONENGINEERING - GA

SOLID DISPOSED WASTE DECAY DATA

\begin{tabular}{|c|c|c|c|c|c|c|}
\hline ACCUMULATED IN & $\begin{array}{r}\text { RADI } \\
\text { AYONA } \\
\text { IDENTIFIED } \\
\text { NUCLIDES }\end{array}$ & $\begin{array}{l}\text { TIVITY } \\
\text { (CURIES ) } \\
\text { UNIDENTIFIED } \\
\text { NUCLIDES }\end{array}$ & \multicolumn{2}{|c|}{$\begin{array}{l}\text { DECAYED VALUE FOR } \\
\text { IDENTIFIED NUCLIDES } \\
\text { THROUGH } 12 / 31 / 94 \\
\text { CURIES REMAINING }\end{array}$} & \multicolumn{2}{|c|}{$\begin{array}{l}\text { DECAYED VALUE FOR } \\
\text { IDENTIFIED NUCLIDES } \\
\text { THROUGH } 12 / 31 / 04 \\
\text { CURIES REMAINING }\end{array}$} \\
\hline 1993 & 1967 & 0 & 1788 & $91 \%$ & 1017 & $52 \%$ \\
\hline TOTALS & 1967 & o & 1788 & $91 \%$ & 1017 & $52 \%$ \\
\hline
\end{tabular}


AIRBORNE EFFLUENT DECAY DATA

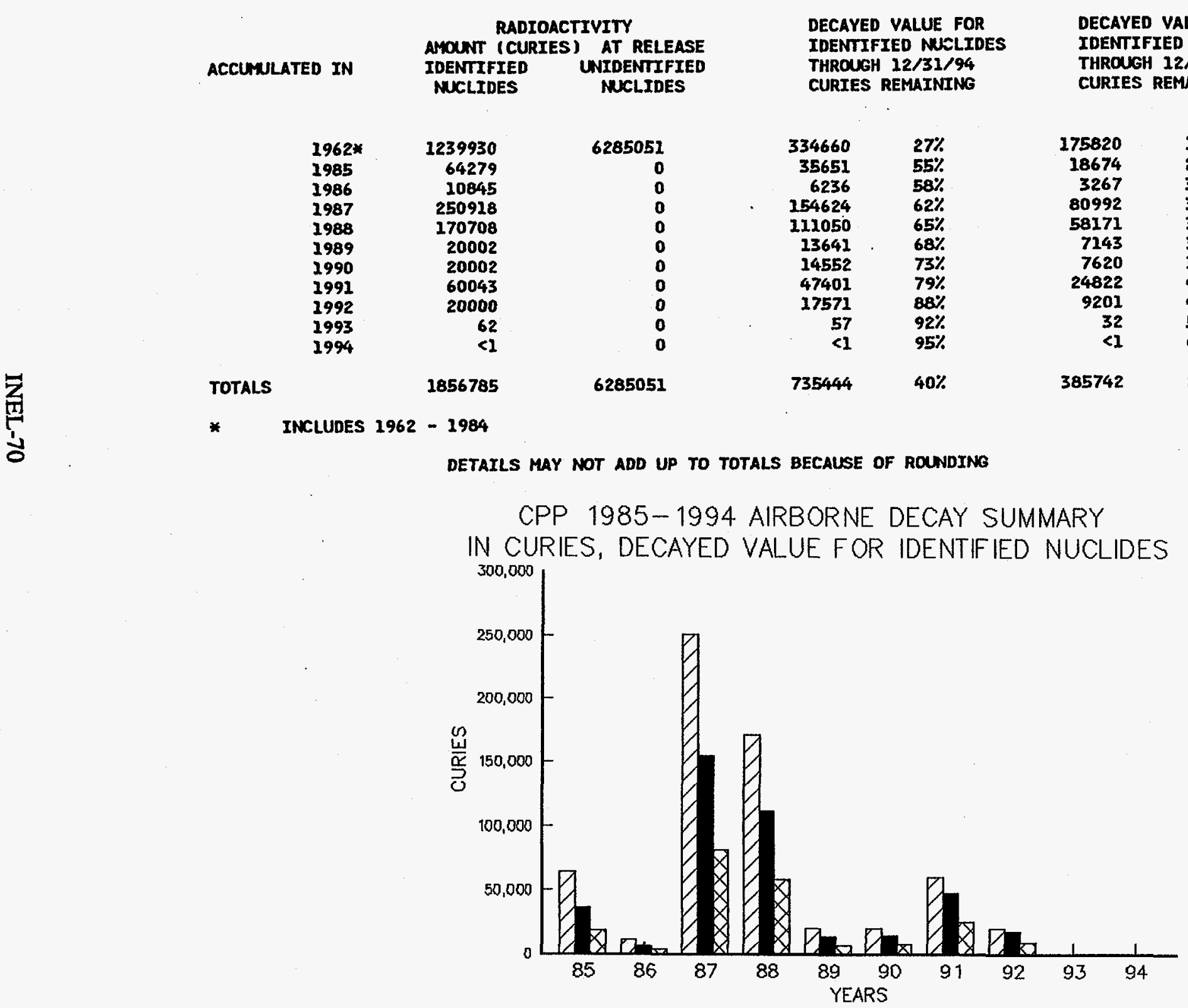

पRELEASED D DECAYED ON 12/31/94 D DECAYED ON 12/31/04 
LIQUID EFFLUENT DATA

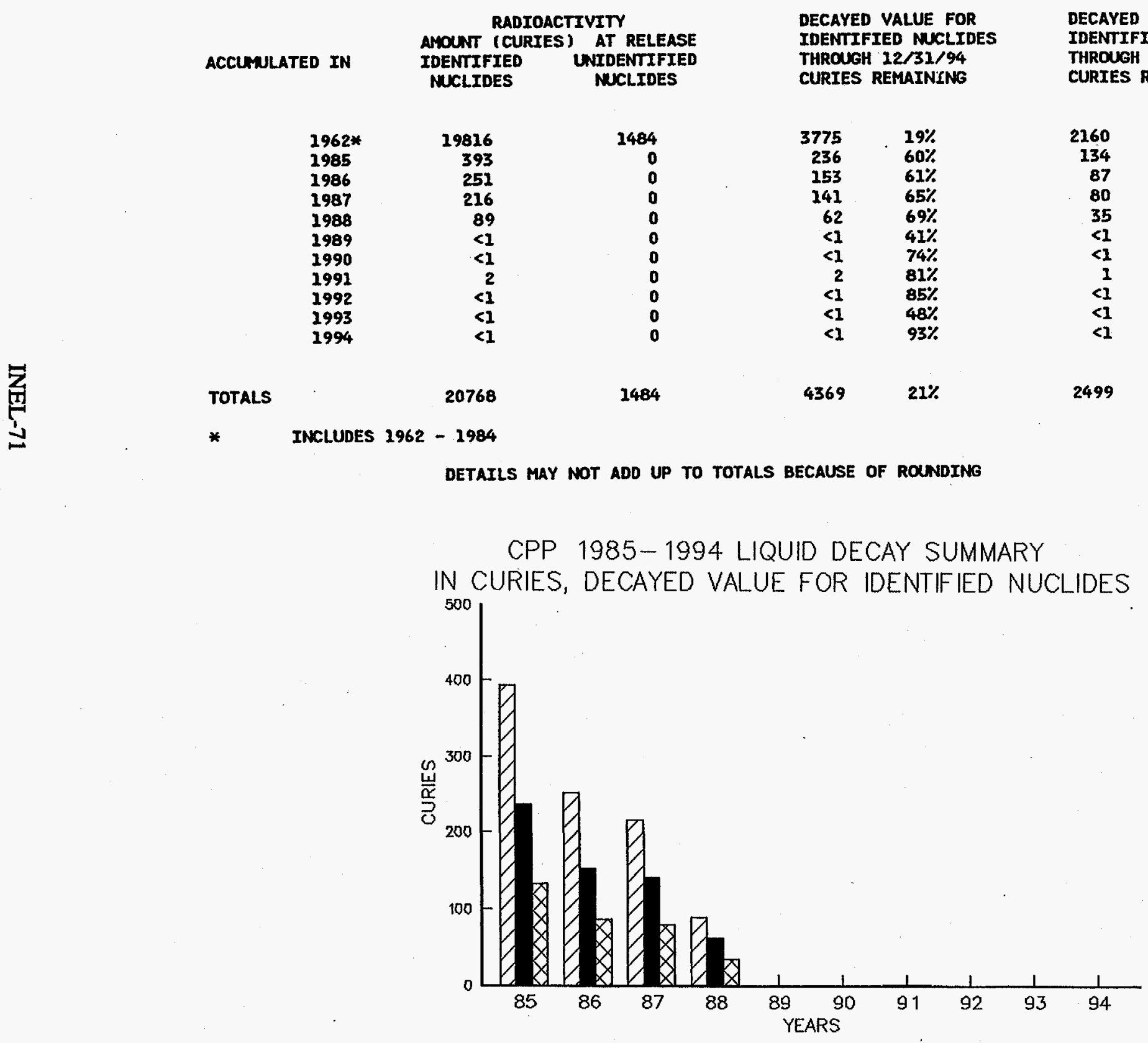

$\square$ DISPOSED DECAYED ON 12/31/94 $\square$ DECAYED ON $12 / 31 / 04$ 
SOLID DISPOSED WASTE DECAY DATA

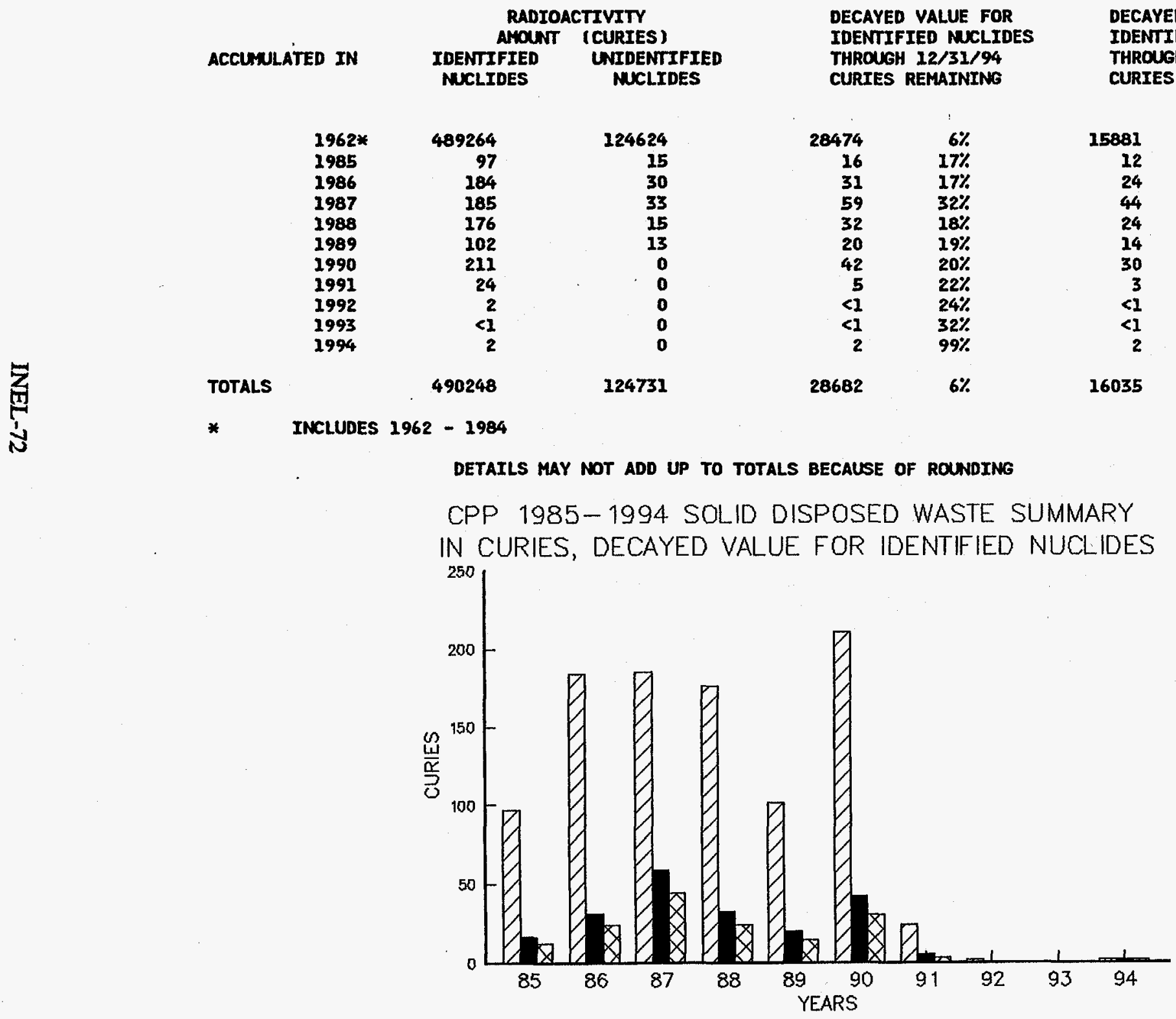

曰DISPOSED DECAYED ON 12/31/94 $₫$ DECAYED ON 12/31/04 
AIRBORNE EFFLUENT DECAY DATA

ACCUMULATEd IN

$$
\begin{aligned}
& \text { RADIOACTIVITY } \\
& \text { AMOUNT (CURIES) AT RELEASE } \\
& \text { IDENIIFIED } \\
& \text { NUCLIDES }
\end{aligned}
$$

1986

1987

TOTALS
DECAYED VALUE FOR IDENTIFIED NUCLIDES THROUEH 12/31/94 CURIES REMAINING

$\begin{array}{rr}<1 & 56 \% \\ 0 & 0 \% \\ & \end{array}$

0

$<1$

$56 \%$

$29 \%$
0

$<1 \quad 29 \%$

DETAILS MAY NOT ADD UP TO TOTALS BECAUSE OF ROUNDING 
LIQUTD EFFLUENT DATA

RADIOACTIVITY

AHOUNT (CURIES) AT RELEASE

IDENTIFIED UNIDENTIFIED

NUCLIDES

DeCAYED VALUE FOR

IDENTIFIED MUCLIDES

THROUGH 12/31/94

CURTES 12/31/94

DECAYEd VALUE for

IDENTIFIEO MUCLIDES

THROUGH 12/31/04

CURIES REMAINING

$\begin{array}{rrrr}<1 & 82 \% & <1 & 65 \% \\ 0 & 0 \% & 0 & 0 \% \\ <1 & 82 \% & <1 & 65 \%\end{array}$

TOTALS

$<1$

$<$ 
ACCUMLATED IN

AMOUNT
(CURIES)
IDENTIFIED
MUCLIDES

1986
1987
1988
1993

TOTALs

*

$\begin{array}{rr}242 & 35 \\ 20 & 6 \\ 0 & <1 \\ <1 & 0\end{array}$

262

35
6
$<1$
0
41

DECAYED VALUE FOR

TOENTIFIED MICLIDES

THROUGH $12 / 31 / 94$

DECAYED VALIUE FOR

IDENTIFIED NUCLIDES

THROUGH 12/31/04

CURIES REMAINING

CURIES REMAINING

$\begin{array}{rr}21 & 9 \% \\ 2 & 10 \% \\ 0 & 0 \% \\ <1 & 58 \%\end{array}$

23

9\%

DETAILS MAY NOT ADD UP TO TOTALS BECAUSE OF ROUNDING

CTF 1985-1994 SOLID DISPOSED WASTE SUMMARY

IN CURIES, DECAYED VALUE FOR IDENTIFIED NUCLIDES

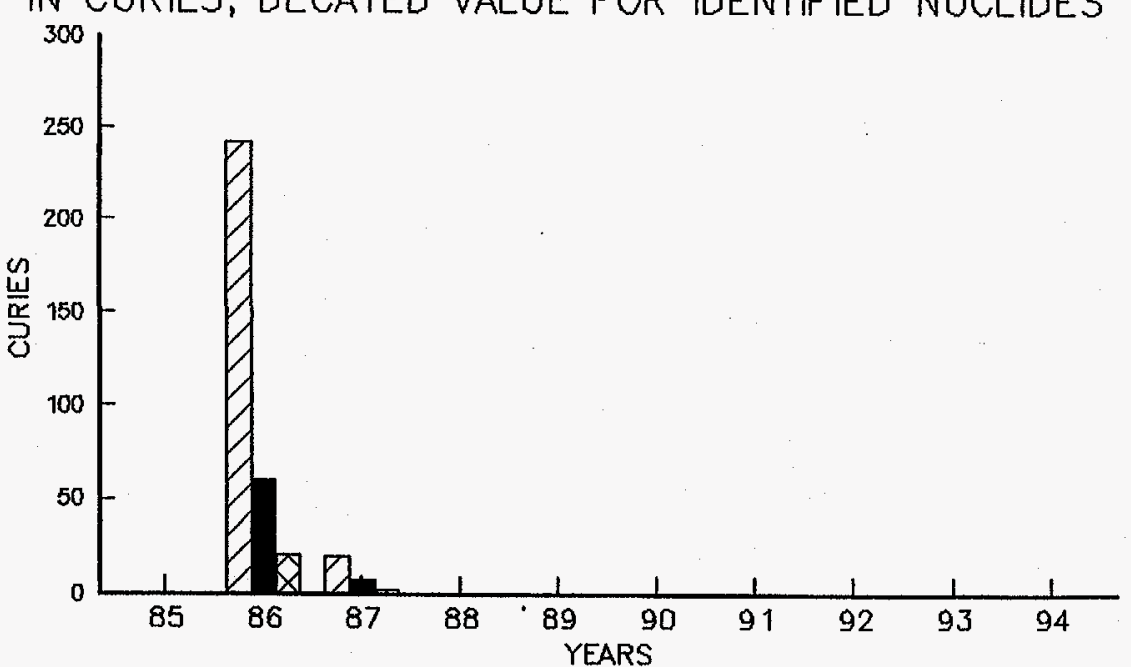

IDISPOSED DECAYED ON 12/31/94 $\triangle D$ DECAYED ON 12/31/04

CTE DISPOSED WASTE IN 86, 87, 88, 93 
SOLID DISPOSED WASTE DECAY DATA

\begin{tabular}{|c|c|c|c|c|c|c|}
\hline \multirow[b]{3}{*}{ ACCUHULATED IN } & \multicolumn{2}{|c|}{ RADIOACTIVITY } & \multicolumn{2}{|c|}{ DECAYED VALUE } & \multicolumn{2}{|c|}{ DECAYED VALUE } \\
\hline & \multicolumn{2}{|c|}{ AMOUNT (CURIES) } & \multirow{2}{*}{\multicolumn{2}{|c|}{$\begin{array}{l}\text { IDENTIFIED NUCLIDES } \\
\text { THROUGH } 12 / 31 / 94 \\
\text { CURIES REMAINING }\end{array}$}} & \multirow{2}{*}{\multicolumn{2}{|c|}{$\begin{array}{l}\text { IDENTIFIED NKC } \\
\text { THROUGH } 12 / 31 / \\
\text { CURIES REMAINI }\end{array}$}} \\
\hline & $\begin{array}{c}\text { IDENTIFIED } \\
\text { NUCLIDES }\end{array}$ & $\begin{array}{l}\text { UNIDENTIFIED } \\
\text { MUCLIDES }\end{array}$ & & & & \\
\hline 1962* & 5632 & 60 & 2755 & $49 \%$ & 1700 & $30 \%$ \\
\hline 1985 & $<1$ & $<1$ & $<1$ & $29 \%$ & $<1$ & $8 \%$ \\
\hline 1986 & $<1$ & $<1$ & $<1$ & $100 \%$ & $<1$ & $100 \%$ \\
\hline 1987 & $<i$ & $<1$ & $<1$ & $67 \%$ & $<1$ & $51 \%$ \\
\hline 1988 & $<1$ & $\mathbf{0}$ & $<1$ & $40 \%$ & $<1$ & $11 \%$ \\
\hline 2989 & $<1$ & 0 & $<1$ & $69 \%$ & $<1$ & $42 \%$ \\
\hline 1990 & $<1$ & $\mathbf{0}$ & $<1$ & $86 \%$ & $<1$ & $67 \%$ \\
\hline 1991 & $<1$ & $\mathbf{0}$ & $<1$ & $90 \%$ & $<1$ & $68 \%$ \\
\hline 1992 & $<\bar{l}$ & 0 & $<1$ & $95 \%$ & $<1$ & $75 \%$ \\
\hline 1993 & 2 & 0 & 2 & $93 \%$ & 1 & $62 \%$ \\
\hline 1994 & $<1$ & $\mathbf{0}$ & $<1$ & $99 \%$ & $<1$ & $74 \%$ \\
\hline TOTALS & 5635 & 60 & 2757 & $49 \%$ & 1701 & $30 \%$ \\
\hline
\end{tabular}

DETAILS MAY NOT ADD UP TO TOTALS BECAUSE OF ROUNDING

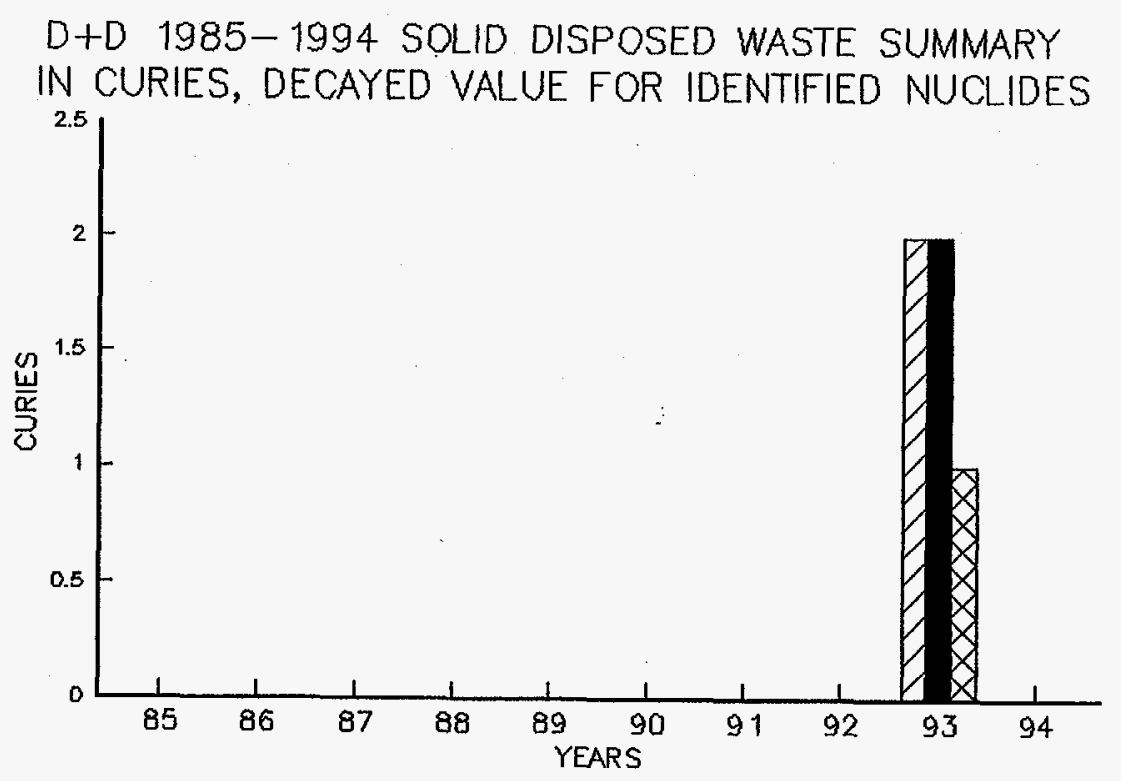

DDISPOSED $\square$ DECAYED ON $12 / 31 / 94$ \& DECAYED ON $12 / 31 / 04$ 
AIRBORNE EFFLUENT DECAY DATA

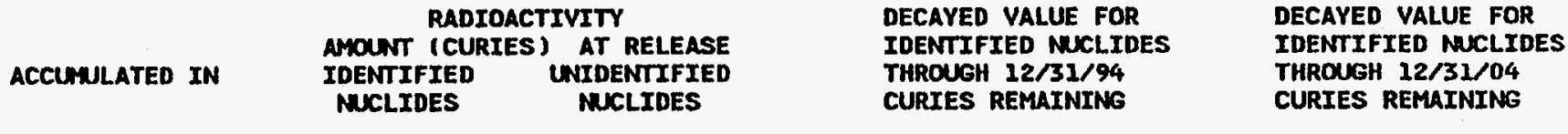

\begin{tabular}{|c|c|c|c|c|c|c|}
\hline $\begin{array}{l}1962 * \\
1985\end{array}$ & $\begin{array}{r}31 \\
8780\end{array}$ & $\begin{array}{r}<1 \\
0\end{array}$ & $\begin{array}{r}<1 \\
6\end{array}$ & $\begin{array}{l}\text { <1\% } \\
\text { <1\% }\end{array}$ & $\begin{array}{r}<1 \\
3\end{array}$ & $\begin{array}{l}<1 \% \\
<1 \%\end{array}$ \\
\hline ALS & 8810 & $<1$ & 6 & $<1 \%$ & 3 & $<1 \%$ \\
\hline
\end{tabular}

* INCLUdes $2962-1984$

DETAILS MAY NOT ADD UP TO TOTALS BECAUSE OF ROUNOING

LOF 1985-1994 AIRBORNE DECAY SUMMARY IN CURIES, DECAYED VALUE FOR IDENTIFIED NUCLIDES

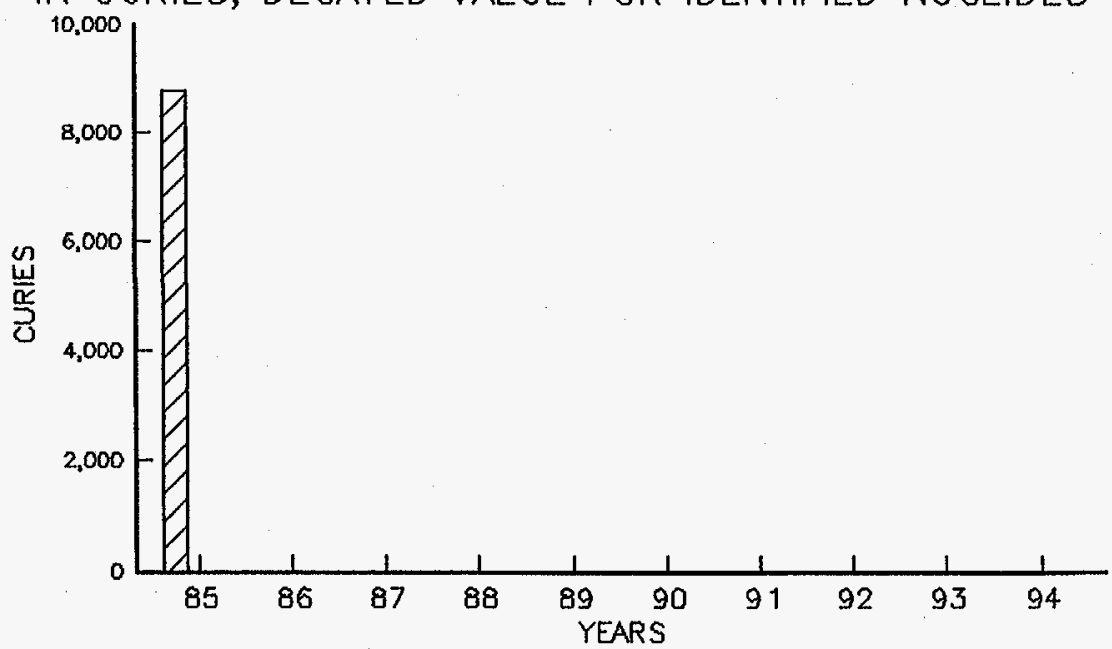

$\square R E L E A S E D \square D E C A Y E D$ ON $12 / 31 / 94$ Q DECAYED ON 12/31/04

LOF RELEASED EFFLUENTS FROM 78-85 
LIQUID EFFLUENT DATA
RADIOACTIVITY

AMOUNT (CURIES) AT RELEASE

IDENTXFIED UNIDENTIFIED

NUCLIDES

DECAYED VALUE FOR IDENTIFIED NUCLIDES

THROUEH 12/31/94

<1 $24 \%$

$<1 \quad 52 \%$

$<1 \quad 37 \%$

$<1$

$<1$

$<1$
CURIES REMATNINO

TOTALS

DECAYED VALUE FOR

IDENTIFIED NUCLIDES

THROUGH $12 / 31 / 04$

* INCLUDES $1962-1984$

DETAILS MAY NOT ADD UP TO TOTALS BECAUSE OF ROUNDING
CURIES REMAINING

$<1 \quad 15 \%$

$<I \quad 25 \%$
$<1 \quad 37 \%$ 


\begin{tabular}{|c|c|c|c|c|c|c|}
\hline ACCUMULATED IN & $\begin{array}{l}\text { RADI } \\
\text { AMOUN } \\
\text { IDENTIFIED } \\
\text { NUCLIDES }\end{array}$ & $\begin{array}{l}\text { TIVITY } \\
\text { (CURIES ) } \\
\text { UNIDENIIFIED } \\
\text { NUCLIDES }\end{array}$ & \multicolumn{2}{|c|}{$\begin{array}{l}\text { DECAYED VALUE FOR } \\
\text { IDENTIFIED NUCLIDES } \\
\text { THROUGH } 12 / 31 / 94 \\
\text { CURIES REMAINING }\end{array}$} & \multicolumn{2}{|c|}{$\begin{array}{l}\text { DECAYED VALUE } \\
\text { IDENTIFIED MK } \\
\text { THROUGH } 12 / 31 / \\
\text { CURTES REMAINT }\end{array}$} \\
\hline $\begin{array}{l}1962 * \\
1985\end{array}$ & $\begin{array}{r}2 \\
<1\end{array}$ & $\begin{array}{l}<1 \\
<1\end{array}$ & $\begin{array}{l}<1 \\
<1\end{array}$ & $\begin{array}{l}12 \% \\
25 \%\end{array}$ & $<1$. & $\begin{array}{r}3 \% \\
15 \%\end{array}$ \\
\hline TOTALS & 2 & $<1$ & $<1$ & $13 \%$ & $<1$ & $4 \%$ \\
\hline
\end{tabular}

DETAILS MAY NOT ADD UP TO TOTALS BECAUSE OF ROUNDING 
AIRBORNE EFFLUENT DECAY DATA

RADTOACTIVITY

AMOUNT (CURIES) AT RELEASE

IDENTIFIED UNIDENTIFIED

MUCLIDES NUCLIDES

勇
品

$1962 *$
1985
1986
1987
1988
1989
1990
1991
1992
1993
1994

1962*

1985

1986

1988

1990

1992

1993

1994

TOTALS

35
$<1$
$<1$
$<1$
$<1$
$<1$
$<1$
$<1$
$<1$
1
2

41
* INCLUDES $1962-1984$
DECAYED VALUE FOR IDENTIFIED NUCLIDES

THROVGH 12/31/94

CURIES REMAINING

$\begin{array}{lrl}<1 & 5 & 13 \% \\ <1 & <1 & 94 \% \\ 0 & <1 & 87 \% \\ 0 & <1 & 84 \% \\ 0 & <1 & 79 \% \\ 0 & <1 & 95 \% \\ 0 & <1 & 94 \% \\ 0 & <1 & 85 \% \\ 0 & <1 & 43 \% \\ 0 & <1 & 75 \% \\ 0 & 1 & 84 \% \\ <1 & & \\ & 23 \%\end{array}$

DETATLS MAY NOT ADD UP TO TOTALS BECAUSE OF ROUNDING
DECAYEd VALUE FOR

IDENTIFIED MUCLIDES

THROUEH 12/31/04

CURIES REMAINING

$\begin{array}{ll}3 & 9 \% \\ <1 & 91 \% \\ <1 & 83 \% \\ <1 & 72 \% \\ <1 & 57 \% \\ <1 & 89 \% \\ <1 & 86 \% \\ <1 & 56 \% \\ <1 & 35 \% \\ <1 & 72 \% \\ <1 & 59 \% \\ 7 & 17 \%\end{array}$




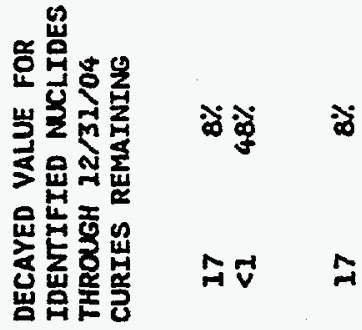

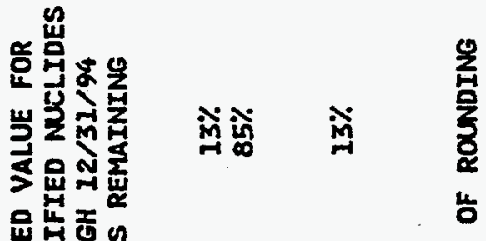

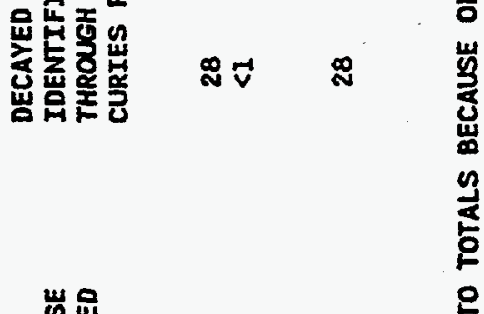

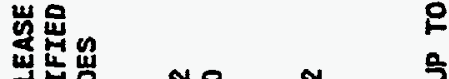

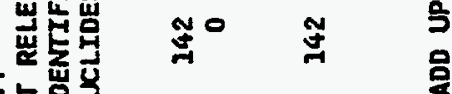

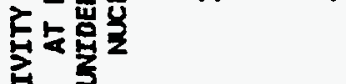

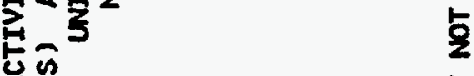

彭哕定

起总脂

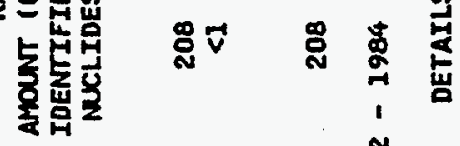

苗 
SOLID DISPOSED WASTE DECAY DATA

ACCUMULATED IN

$\begin{array}{lr}1962 * & 920638 \\ 1985 & 141754 \\ 1986 & 35930 \\ 1987 & 29665 \\ 1988 & 6732 \\ 1989 & 126487 \\ 1990 & 74089 \\ 1991 & 102852 \\ 1992 & 49795 \\ 1993 & 42260 \\ 1994 & 36901\end{array}$

RADIOACTIVITY.

\begin{tabular}{cc}
\multicolumn{2}{c}{ RADIOACTIVITY. } \\
AMOUNT \\
(CURIES) \\
IDENTIFIED & UNIDENTIFIED \\
MUCLIDES & MUCLIDES
\end{tabular}

1567097

3095387
0
0
0
0
$<1$
0
0
0
0
0

3095387
DECAYED YALUE FOR IDENTIFIED MUCLIDES THROUGH 12/31/94 CURIES REMAINING

260501
79930
21039
17989
4271
91150
49207
53242
34796
35643
34680

79930

1039

4271

5324

34680

682446

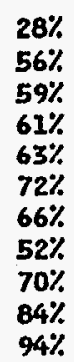

$28 \%$
$56 \%$
$59 \%$
$61 \%$
$63 \%$
$72 \%$
$66 \%$
$52 \%$
$70 \%$
$84 \%$
$94 \%$

DECAYED VALUE FOR IDENTIFIED MUCLIDES THROUGH 12/31/04 CURIES REMAINING

$\begin{array}{rr}203922 & 22 \% \\ 64288 & 45 \% \\ 16553 & 46 \% \\ 13859 & 47 \% \\ 3202 & 48 \% \\ 71015 & 56 \% \\ 34972 & 47 \% \\ 35449 & 34 \% \\ 23457 & 47 \% \\ 24130 & 57 \% \\ 22982 & 62 \%\end{array}$

$513829 \quad 33 \%$

* INCLUdes 1962 - 2984

DETAILS MAY NOT ADD UP TO TOTALS BECAUSE OF ROUNDING

NRF 1985-1994 SOLID DISPOSED WASTE SUMMARY IN CURIES, DECAYED VALUE FOR IDENTIFIED NUCLIDES

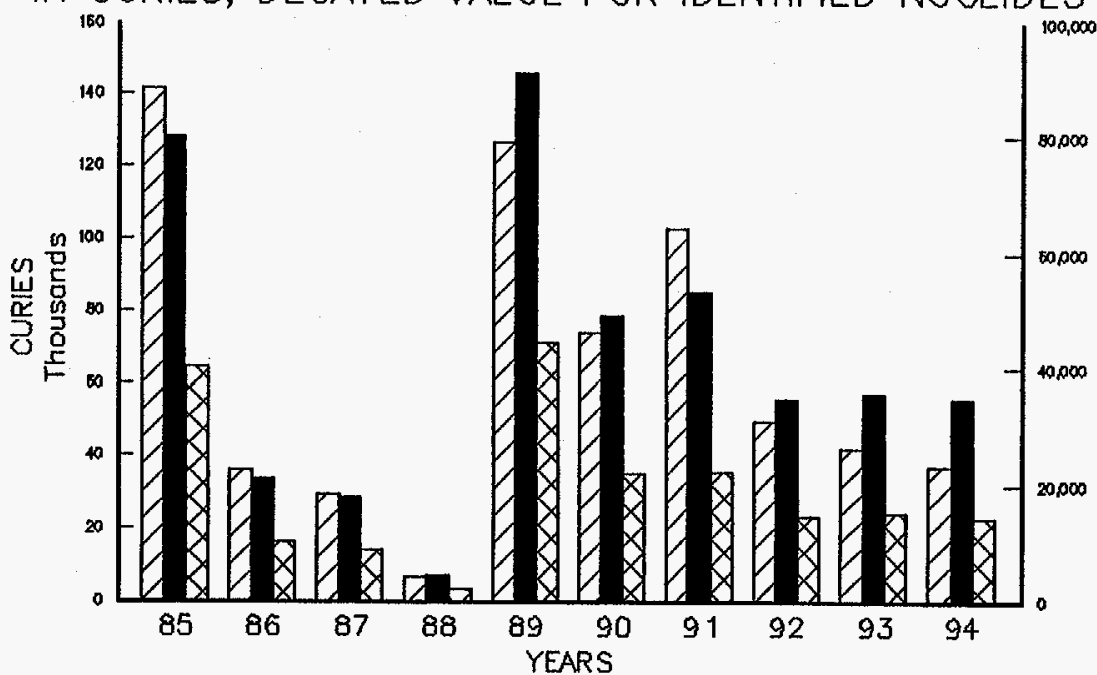

I DISPOSED DDECAYED ON 12/31/94 D DECAYED ON 12/31/04 
AIRBORNE EFFLUENT DECAY DATA

$\begin{array}{llll} & \text { RADIOACTIVITY } & \text { DECAYED VALUE FOR } & \text { DECAYED VALUE FOR } \\ \text { ACCUMLATED IN } & \text { AMOUNT (CURIES) AT RELEASE } & \text { IDENTFIED MUCLIDES } & \text { IDENIFIED NUCLIDES } \\ \text { IDENTIFIED } & \text { UNIDENIFIED } & \text { THROUGH } 12 / 31 / 94 & \text { THROUGH } 12 / 31 / 04 \\ \text { NUCLIDES } & \text { NUCLIDES } & \text { CURIES REMAINING } & \text { CURIES REMAINING }\end{array}$

$\begin{array}{llrrrrr}1962 * & <1 & <1 & <1 & 27 \% & <1 & 22 \% \\ 1985 & <1 & <1 & <1 & 30 \% & <1 & 24 \% \\ 1986 & <1 & <1 & <1 & 47 \% & <1 & 37 \% \\ 1987 & <1 & 0 & 0 & 0 \% & 0 & 0 \% \\ 1988 & <1 & 01 & <1 & 49 \% & <1 & 4 \% \\ 1989 & <1 & 01 & 01 & 4 \% & <1 & 39 \% \\ 1990 & <1 & 0 & <1 & 45 \% & <1 & 3 \% \\ 1991 & <1 & 0 & <1 & 28 \% & <1 & 36 \% \\ 1992 & <1 & 0 & <1 & <1 \% & <1 & 22 \% \\ 1993 & <1 & 0 & <1 & 8 \% & <1 & <1 \% \\ 1994 & <1 & 0 & <1 & 27 \% & <1 & 6 \% \\ & <1 & & & <1 & 22 \%\end{array}$

* INClUdes $1962-1984$

DETAILS MAY NOT ADD UP TO TOTALS BECAUSE OF ROUNDING 


\section{LIQUID EFFLUENT DATA}

RADIOACTIVITY

AMOUNT (CURIES) AT RELEASE

IDENTIFIED UNIDENTIFIED

NUCLIDES

DECAYED VALUE FOR

IOENTIFIED NUCLIDES

THROUGH 12/31/94

CURIES REMAINING

$<1$

$\therefore \%$

$<1$

$48 \%$

TOTALS

$<1$

$<1$

* INCLUDES $1962-1984$
DECAYED VALUE FOR

IDENTIFIED MUCLIDES

THROUGH 12/31/04

CURIES REMAINING

DETAILS MAY NOT ADD UP TO TOTALS BECAUSE OF ROUNDING 
SOLID DISPOSED WASTE DECAY DATA

\begin{tabular}{|c|c|c|c|c|}
\hline & RADI & ctrVITY & DECAYED VALUE FOR & DECAYED VALUE FOR \\
\hline CCUMLATED IN & $\begin{array}{l}\text { AMON } \\
\text { IDENTIFIEO } \\
\text { MUCLIOES }\end{array}$ & $\begin{array}{l}\text { (CURIES) } \\
\text { UNIDENTIFIED } \\
\text { NUCLIDES }\end{array}$ & $\begin{array}{l}\text { IDENTIFIED NUCLIDES } \\
\text { THROUGH } 12 / 31 / 94 \\
\text { CURXES REMAINING }\end{array}$ & $\begin{array}{l}\text { IDENTIFIED NEKLIDES } \\
\text { THROUGH } 12 / 31 / 04 \\
\text { CURIES RENHANING }\end{array}$ \\
\hline
\end{tabular}

\begin{tabular}{|c|c|c|c|c|c|}
\hline $\begin{array}{l}1962 * \\
1985 \\
1986 \\
1987 \\
1988 \\
1994\end{array}$ & $\begin{array}{r}<1 \\
367 \\
0 \\
8 \\
<1 \\
<1\end{array}$ & $\begin{array}{r}188 \\
4 \\
<1 \\
3 \\
2 \\
0\end{array}$ & $\begin{array}{r}<1 \\
256 \\
0 \\
3 \\
<1 \\
<1\end{array}$ & $\begin{array}{r}94 \% \\
70 \% \\
0 \% \\
39 \% \\
41 \% \\
99 \%\end{array}$ & $\begin{array}{r}<1 \\
202 \\
0 \\
<1 \\
<1 \\
<1\end{array}$ \\
\hline & 376 & 197 & 260 & $69 \%$ & 203 \\
\hline
\end{tabular}

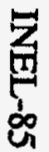

DETAILS MAY NOT ADD UP TO TOTALS BECAUSE OF ROUNDTNG

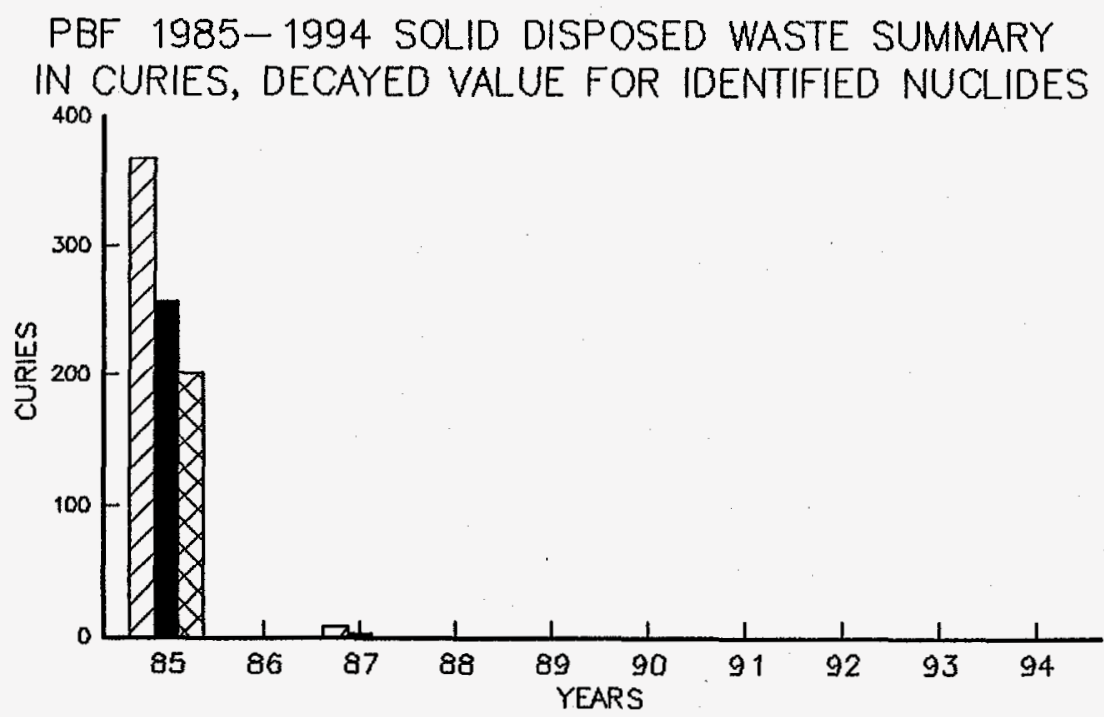

DDISPOSED DDECAYED ON 12/31/94 ØDECAYED ON 12/31/04

PBF HAD NO IDENTIFIED NUCLIDES IN 81,83,
84,86 OR 88 THRU 93 


\section{SOLID DISPOSED WASTE DECAY DATA}

\begin{tabular}{|c|c|c|c|c|}
\hline \multirow[b]{3}{*}{ ACCUNULATED IN } & \multicolumn{2}{|c|}{ RADIOACTIVITY } & \multirow{2}{*}{\multicolumn{2}{|c|}{$\begin{array}{l}\text { DECAYED VALUE FOR } \\
\text { IDENTIFIED NUCLIDES }\end{array}$}} \\
\hline & AMtoun & (CURIES) & & \\
\hline & $\begin{array}{l}\text { IDENTIFIED } \\
\text { MUCLIDES }\end{array}$ & $\begin{array}{l}\text { UNIDENTIFIED } \\
\text { NUCLIDES }\end{array}$ & $\begin{array}{l}\text { THRO } \\
\text { CURI }\end{array}$ & $\begin{array}{l}2 / 31 / 94 \\
\text { MAINING }\end{array}$ \\
\hline 1962* & 4870 & 251140 & 4790 & $98 \%$ \\
\hline TOTALS & 4870 & 251140 & 4790 & $98 \%$ \\
\hline
\end{tabular}

DETAILS MAY NOT ADD UP TO TOTALS BECAUSE OF ROUNDING
DECAYEd VALUE FOR

IDENTIFIED NUCLIDES

THROUGH 12/31/04

CURIES REMAINING

$4784 \quad 98 \%$

$478498 \%$ 
AIRBORNE EFFLUENT DECAY DATA

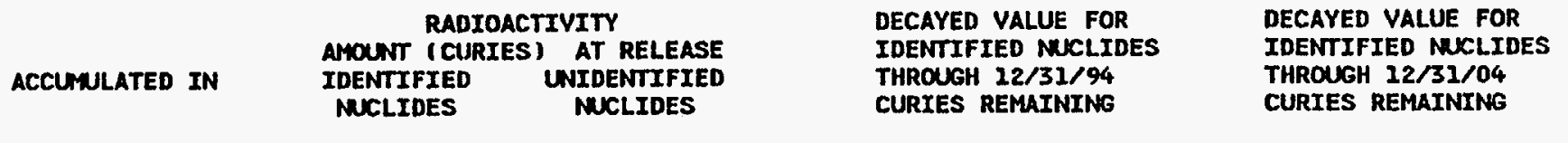

$\begin{array}{lllllll}1985 & <1 & 0 & <1 & 43 \% & <1 & 43 \% \\ 1986 & <1 & 0 & <1 & 43 \% & <1 & 43 \% \\ 1987 & <1 & 0 & <1 & 22 \% & <1 & 22 \% \\ 1988 & <1 & 0 & <1 & 43 \% & <1 & 43 \% \\ 1989 & <1 & 01 & <1 & 36 \% & <1 & 36 \% \\ 1990 & <1 & 0 & <1 & 36 \% & <1 & 36 \% \\ 1991 & <1 & 0 & <1 & 36 \% \\ 1992 & <1 & 0 & <1 & 36 \% & <1 & 36 \% \\ 1993 & <1 & 0 & <1 & 37 \% & <1 & 36 \% \\ 1994 & <1 & 0 & <1 & 30 \% & <1 & 36 \% \\ & <1 & 0 & 0 & & <1 & 30 \%\end{array}$

INKLUDES 1962 - 1984

DETAILS MAY NOT ADD UP TO TOTALS BECALSE OF ROUNDING 
SOLID DISPOSED MASTE DECAY DATA

$\begin{array}{ccl} & \text { RADIOACTIVITY } & \text { DECAYED VALUE FOR } \\ \text { ACCUNULATED IN } & \text { AMONT (CURIES) } & \text { IDENTIFIED NUCLIDES } \\ \text { IDENTIFIED } & \text { UNIDENTIFIED } & \text { THROUGH } 12 / 31 / 94 \\ \text { NUCLIDES } & \text { NUCLIDES } & \text { CURIES REMAINING }\end{array}$

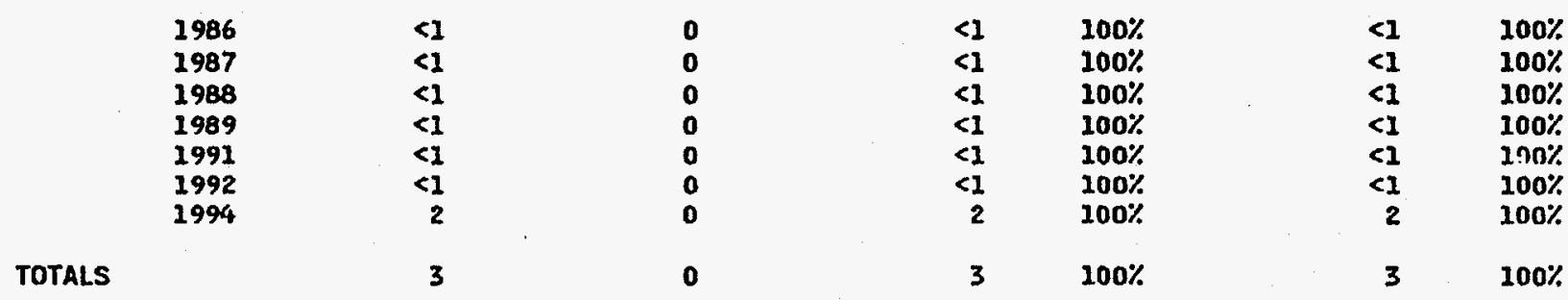

* INCLUDES $1962-1984$

DETAILS MAY NOT ADD UP TO TOTALS BECAUSE OF ROUNDING 
AIRBORNE EFFLUENT DECAY DATA

ACCUNULATED IN

2962*

TOTALS

\section{RADIOACTIVITY}

AHOUNT (CURIES) AT RELEASE

ADENTIFIED * LNIDENTIFIED

NUCLIDES

$\mathbf{0}$

o
6
DECAYED VALUE FOR IDENTIFIED NUCLIDES

TDENIIFIED NUCLIDES

THROUGH $12 / 31 / 94$

CURIES REMAINING

D $\quad 0 \%$

0 $\%$
DECAYED VALUE FOR IDENTIFIED MUCLIDES

TOENTFIED NUCLIOES

THROU $12 / 31 / 04$

* INCLUDES $1962-1984$

DETAILS MAY NOT ADD UP TO TOTALS BECAUSE OF ROUNDING 
LIOUIO EFFLUENT DATA

RADIOACTIVITY

AMOUNT (CURIES) AT RELEASE

IDENTIFIED UNIDENTIFIED

MUCLIDES
DECAYEd VALUE FOR
IDENTXFIED MUCLIDES
THROUGH 12/31/94

CURIES REMAINING

1962*

$<1$

155

155

$<1$

$28 \%$

$<1$

$28 \%$

TOTALS

$<1$

INCLUDES $1962-1984$
DECAYED YALUE FOR

IDENTIFIED NUCLIDES

THROUGH 12/31/04

CURIES REMAINING

$<1 \quad 22 \%$

$<1 \quad 22 \%$ 
SOLIO DISPOSED MASTE DECAY DATA

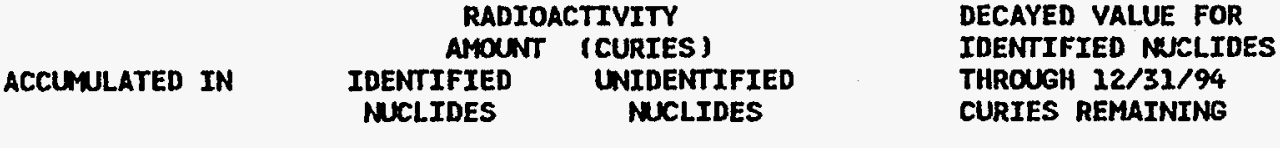

DECAYED VALUE FOR

IDENTIFIED MULLIDES

THROUGH $12 / 31 / 04$

CURIES REMAINING

\begin{tabular}{|c|c|c|c|c|c|c|}
\hline $\begin{array}{l}1962 * \\
1987 \\
1989 \\
1991\end{array}$ & $\begin{array}{r}5 \\
<1 \\
0 \\
<1\end{array}$ & $\begin{array}{r}19 \\
<1 \\
<1 \\
0\end{array}$ & $\begin{array}{r}<1 \\
<1 \\
0 \\
<1\end{array}$ & $\begin{array}{r}9 \% \\
100 \% \\
0 \% \\
76 \%\end{array}$ & $\begin{array}{r}<1 \\
<1 \\
0 \\
<1\end{array}$ & $\begin{array}{r}6 \% \\
100 \% \\
0 \% \\
46 \%\end{array}$ \\
\hline & 5 & 19 & $<I$ & $9 \%$ & $<1$ & $6 \%$ \\
\hline
\end{tabular}

TOTALS

INCLUDES $1962-1984$

DETAILS MAY NOT ADD UP TO TOTALS BECAUSE OF ROUNDING 
SCHEDULE NO. PG1PMO21-B

TEST AREA NORTH

AIRBORNE EFFLUENT DECAY DATA

ACCUMULATED IN

$$
\begin{aligned}
& 1962 * \\
& 1985 \\
& 1986 \\
& 1987 \\
& 1988 \\
& 1989 \\
& 1990 \\
& 1991 \\
& 1992 \\
& 1993 \\
& 1994
\end{aligned}
$$

TOTALS

*

INCLUDES $1962-1984$
RADIOACTIVITY

AMOUNT (CURIES) AT RELEASE IDENTIFIED UNIDENTIFIED NUCLIDES

DECAYED VALUE FOR IDENTIFIED MUCLIDES

THROUGH 12/31/94

CURIES REMAINING

53724
$<1$
$<1$
0
0
0
0
0
0
0
0

53724

$\begin{array}{rr}<1 & 45 \% \\ <1 & 41 \% \\ <1 & 9 \% \\ <1 & 52 \% \\ <1 & 31 \% \\ <1 & 44 \% \\ <1 & 18 \% \\ <1 & 17 \% \\ <1 & <1 \% \\ <1 & 31 \% \\ <1 & 24 \%\end{array}$

DECAYED VALUE FOR

IDENTIFIED NUCLIDES

THROUGH 12/31/04

CURIES REMAINING

DETAILS MAY NOT ADD UP TO TOTALS BECAUSE OF ROUNOING

$\begin{array}{ll}<1 & 24 \% \\ <1 & 33 \% \\ <1 & 7 \% \\ <1 & 31 \% \\ <1 & 25 \% \\ <1 & 35 \% \\ <1 & 14 \% \\ <1 & 14 \% \\ <1 & <1 \% \\ <1 & 22 \% \\ <1 & 19 \%\end{array}$

$<1 \quad 24 \%$ 
LIQUID EFFLUENT DATA

RADIOACTIVITY

AMOUNT (CURIES) AT RELEASE

TDENITIFIED UNIDENTIFIED

NUCLIDES
DECAYED VALUE FOR
IDENTIFIED MUXLIDES
THROUGH 12/31/94

CURIES REMAINING

TOTALS

章

$\begin{array}{ll}19 & 39 \\ <1 & <1 \\ <1 & 0 \\ <1 & 0 \\ <1 & 0 \\ <1 & 0 \\ <1 & 0 \\ <1 & 0 \\ <1 & 0 \\ 2 & 0\end{array}$

22

39

$1962 *$
1985
1986
1987
1988
1989
1990
1991
1992
1993

6
$<1$
$<1$
$<1$
$<1$
$<1$
$<1$
$<1$
$<1$
2

$30 \%$

$56 \%$

$60 \%$

$<1 \%$

$66 \%$

$64 \%$

$80 \%$

$83 \%$

$92 \%$

$36 \%$

INCLUDES $1962-1984$
DETATLS MAY NOT ADD UP TO TOTALS BECAUSE OF ROUNDING
DECAYED VALUE FOR IDENTIFIED NUCLIDES THROVGH $12 / 31 / 04$ CURIES REMATNING

$\begin{array}{rr}3 & 17 \% \\ <1 & 32 \% \\ <1 & 35 \% \\ <1 & <1 \% \\ <1 & 5 \% \\ <1 & 38 \% \\ <1 & 37 \% \\ <1 & 46 \% \\ <1 & 47 \% \\ <1 & 52 \%\end{array}$

5
$17 \%$
$32 \%$
$35 \%$
$<1 \%$
$5 \%$
$38 \%$
$37 \%$
$46 \%$
$47 \%$
$52 \%$

$21 \%$ 
SOLID DISPOSED WASTE DECAY DATA

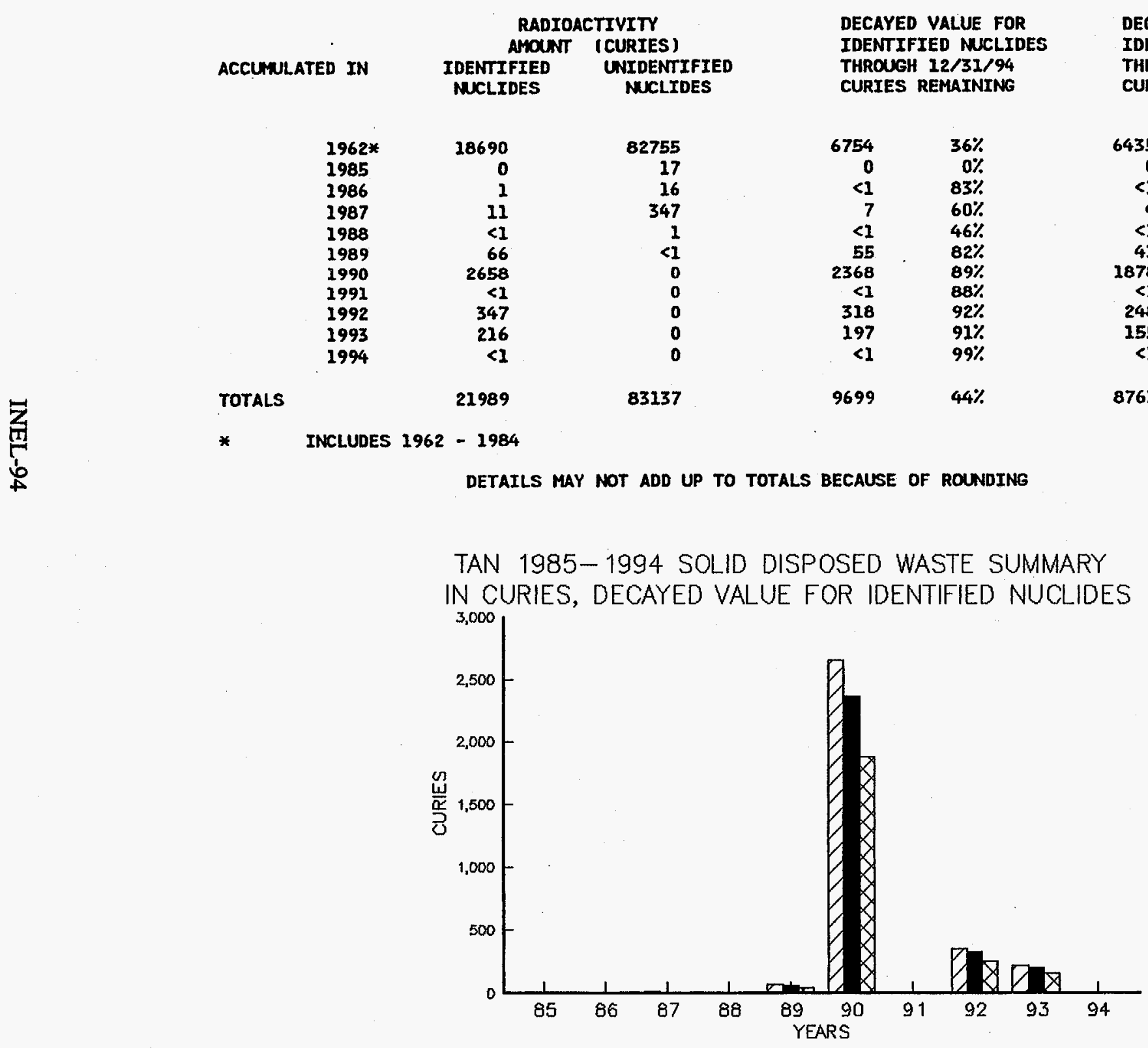

口DISPOSED DECAYED ON 12/31/94 \DECAYED ON 12/31/04 
AIRBORNE EFFLUENT DECAY DATA

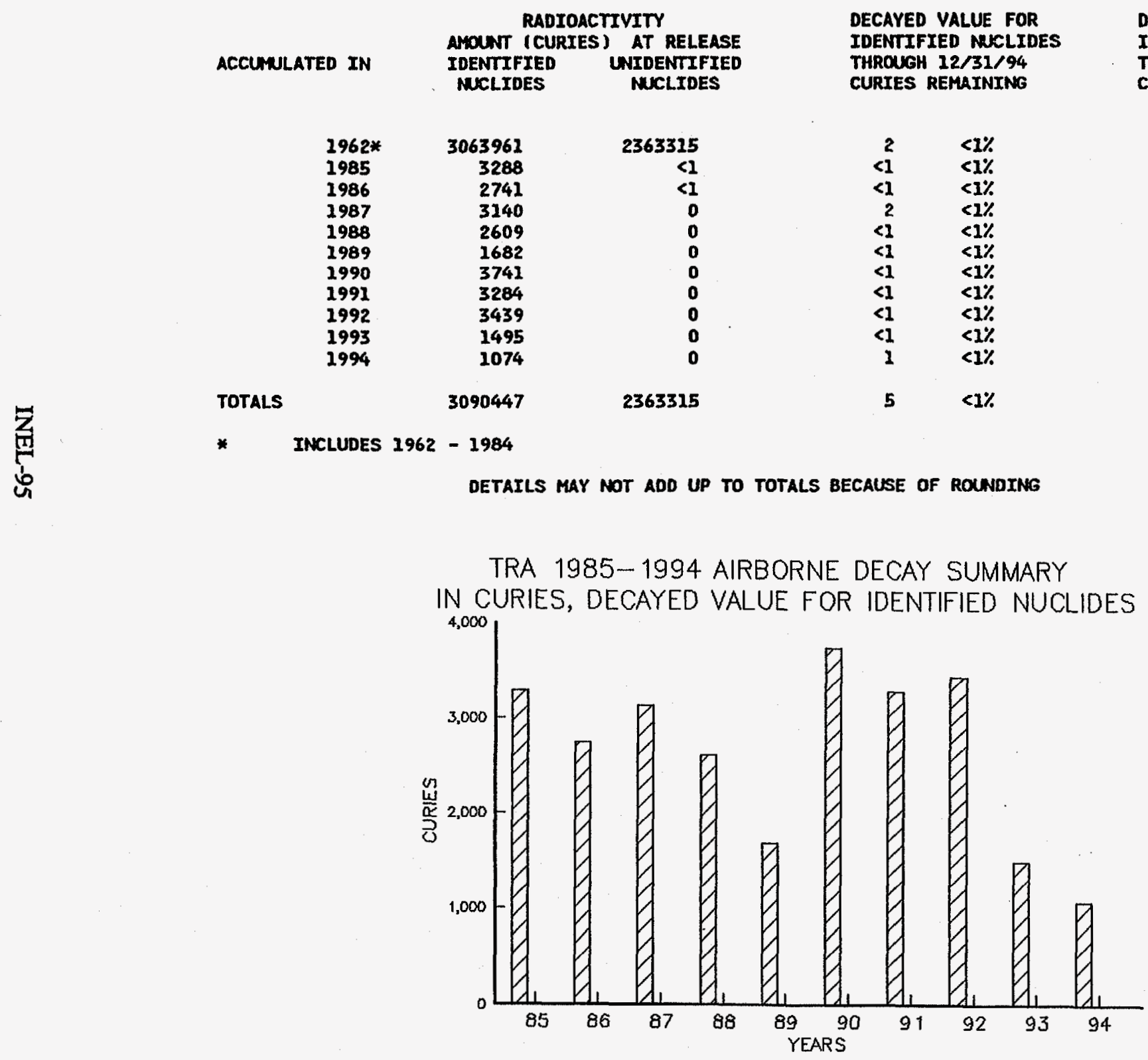

$\square$ RELEASED $\square$ DECAYED ON 12/31/94 $\square$ DECAYED ON $12 / 31 / 04$

DECAYED VALUE FOR

DENTIFIED MCLIDES

THROUGH 12/31/04

CURIES REMAINING

$\begin{array}{ll}<1 & <1 \% \\ <1 & <1 \% \\ <1 & <1 \% \\ <1 & <1 \% \\ <1 & <1 \% \\ <1 & <1 \% \\ <1 & <1 \% \\ <1 & <1 \% \\ <1 & <1 \% \\ <1 & <1 \% \\ <1 & <1 \% \\ 2 & <1 \%\end{array}$


LIQUTD EFFLUENT DATA

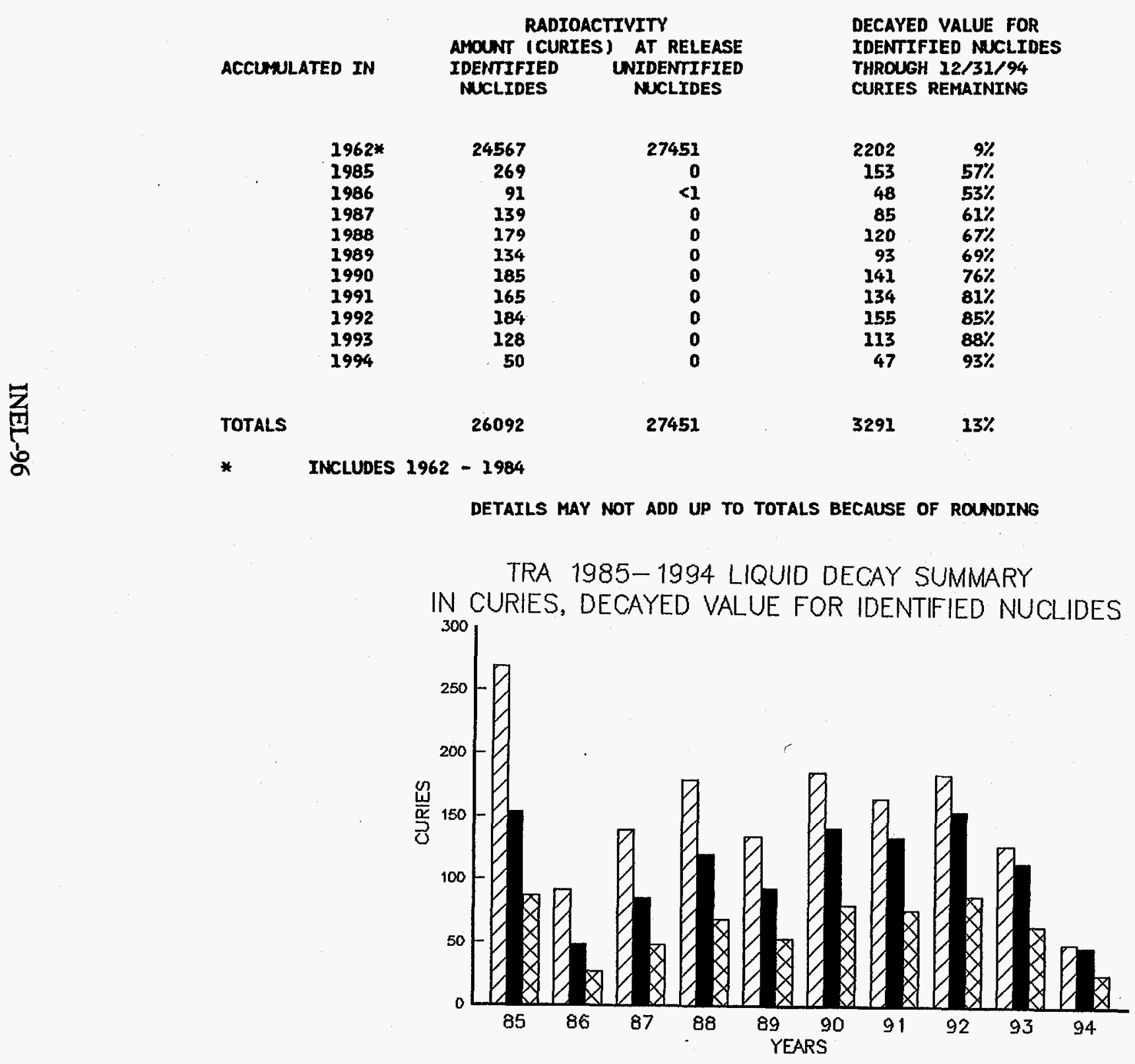

DECAYED VALUE FOR

IDENTIFIED MUCLIDES

THROUGH 12/31/04

CURIES REMAINIAH

$\begin{array}{rr}1276 & 5 \% \\ 87 & 32 \% \\ 27 & 30 \% \\ 48 & 35 \% \\ 68 & 38 \% \\ 53 & 39 \% \\ 80 & 43 \% \\ 76 & 46 \% \\ 88 & 48 \% \\ 64 & 50 \% \\ 26 & 52 \%\end{array}$

$1895 \quad 7 \%$

DETAILS MAY NOT ADD UP TO TOTALS BECAUSE OF ROUNDING

TRA 1985-1994 LIQUID DECAY SUMMARY

$\square$ RELEASED DECAYED ON $12 / 31 / 94$ D DECAYED ON $12 / 31 / 04$ 
SOLID DISPOSED MASTE DECAY DATA

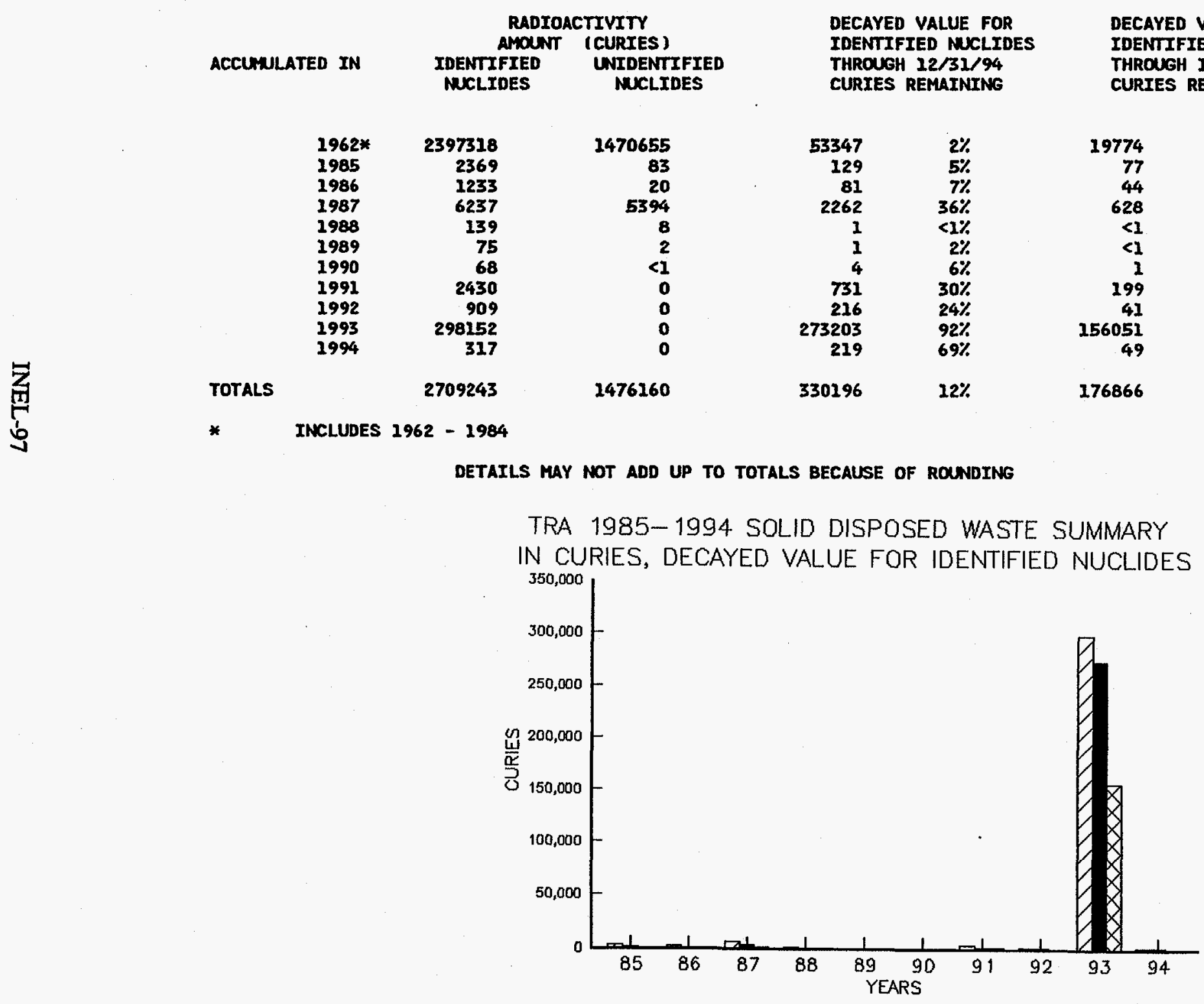

$\square D I S P O S E D \square D E C A Y E D$ ON 12/31/94 DDECAYED ON 12/31/04 


\section{AIRBORNE EFFLUENT DECAY DATA}

RADIOACTIVITY

AMOUNT (CURIES) AT RELEASE

IDENTIFIED UNIDENTIFIED

NUCLIDES

DECAYED VALUE FOR IDENTIFIED NUCLIDES

THROUGH 12/31/94

CURIES REMAINING

DECAYED VALUE FOR

IDENTIFIED MUCLIDES

THROUGH 12/31/04

CURIES REMAINTNG

\begin{tabular}{|c|c|c|c|c|c|c|}
\hline 1986 & $<1$ & $<1$ & $<1$ & $26 \%$ & $<1$ & 2.1\% \\
\hline 1987 & $<1$ & 0 & $<1$ & $32 \%$ & $<1$ & $25 \%$ \\
\hline 1988 & $<1$ & 0 & $<1$ & $31 \%$ & $<1$ & $24 \%$ \\
\hline 1989 & $<1$ & 0 & $<1$ & $6 \%$ & $<I$ & $5 \%$ \\
\hline 2990 & $<1$ & 0 & $<1$ & $9 \%$ & $<1$ & $7 \%$ \\
\hline 1991 & $<1$ & 0 & $<1$ & $38 \%$ & $<1$ & $28 \%$ \\
\hline 1992 & $<1$ & 0 & $<1$ & $7 \%$ & $<1$ & $6 \%$ \\
\hline 1993 & 0 & 0 & 0 & $49 \%$ & 0 & $38 \%$ \\
\hline 1994 & $<1$ & 0 & $<1$ & $95 \%$ & $<1$ & $75 \%$ \\
\hline & $<1$ & $<1$ & $<1$ & $30 \%$ & $<1$ & $\%$ \\
\hline
\end{tabular}

\section{TOtals}

DETAILS MAY NOT ADD UP TO TOTALS BECAUSE OF ROUNDING 
WASTE EXPERLFENIAL REUUCTIUNTACTILIY

SOLID DTSPOSED WASTE DECAY DATA

ACCUNULATED IN

RADIOACTIVITY
AMOUNT
(CURIES)
IDENTIFIED
UNIDENTIFIED
NUCLIDES

TOTALS

$$
\begin{aligned}
& 1985 \\
& 1986 \\
& 1987 \\
& 1988 \\
& 1989 \\
& 1990 \\
& 1991 \\
& 1993
\end{aligned}
$$

* INCLUdes $1962-1984$

$\begin{array}{rr}0 & <1 \\ 0 & <1 \\ 0 & <1 \\ 0 & <1 \\ 0 & <1 \\ 0 & 6 \\ 0 & 3 \\ <1 & 0 \\ <1 & 0 \\ <1 & \\ <1 & 10\end{array}$

DECAYED VALUE FOR IDENTIFIED MCLIDES THROUGH $12 / 31 / 94$

$\begin{array}{rr}0 & 0 \% \\ 0 & 0 \% \\ 0 & 0 \% \\ 0 & 0 \% \\ 0 & 0 \% \\ 0 & 0 \% \\ 0 & 0 \% \\ <1 & 87 \% \\ <1 & 100 \% \\ <1 & 99 \%\end{array}$

DETAILS MAY NOT ADD UP TO TOTALS BECAUSE OF ROUNDING
CURIES REMAINING

DECAYED VALUE FOR IDENTIFIED MUCLIDES THROUGH 12/31/04 CURIES REMAINING 
ATPBORNE EFFLUENT DECAY DATA

RADIOACTIVITY

AHOUNT (CURIES) AT RELEASE

IDENTIFIED UNIDENTIFIED

MXLIDES

AMCLIDES

DECAYED VALUE FOR IDENTIFIED NUCLIDES

THROUGH 12/31/94

CURTES REMAINING

1987
1988
1989
1992
1993

TOTALS

$\begin{array}{llrr}<1 & 0 & <1 & 3 \% \\ <1 & 0 & 0 & 0 \% \\ <1 & 0 & 0 & 0 \% \\ <1 & 0 & 0 & 0 \% \\ <1 & 0 & 0 & 0 \% \\ <1 & 0 & <1 & <1 \%\end{array}$

DECAYED VALUE FOR

IDENTIFIED NCLIDES

THROUGH 12/31/04

CURIES REMAINING

$\begin{array}{ll}<1 & 2 \% \\ 0 & 0 \% \\ 0 & 0 \% \\ 0 & 0 \% \\ 0 & 0 \% \\ <1 & <1 \%\end{array}$

DETAILS MAY NOT ADD UP TO TOTALS BECAUSE OF ROUNDING 
LIQUID EFFLUENT DATA

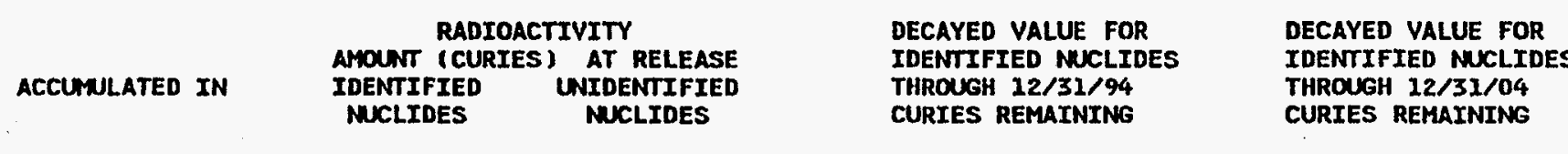

0

0

$0 \%$

0

$0 \%$

TOTALS

o

o

$\%$

0

INCLUUDES 1962 - 1984

DETAILS MAY NOT ADD UP TO TOTALS BECAUSE OF ROUNOING 


\section{haste managemeNT COMPLEX}

RUN DATE: 05/02/95

SOLID DISPOSED WASTE DECAY DATA

\section{accuntateo IN}

$1962 x$
1986
1987
1989
1990

TOTALS

*

\section{RADIOACTIVITY \\ AMOUNT (CURIES)
IDENTIFIED
NIDENTIFIED MUCLIDES}

60920

$$
\begin{aligned}
& 6 \\
& <1 \\
& 83 \\
& <1 \\
& <1
\end{aligned}
$$

89
60920
0
$<1$
0
0

60920
DECAYED VALUE FOR IDENTIFIED MKLIDES

THROUGH 12/31/94

CURIES REMAINING

$\begin{array}{rr}4 & 67 \% \\ <1 & 99 \% \\ 82 & 99 \% \\ <1 & 100 \% \\ <1 & 100 \%\end{array}$

86
DECAYED VALUE FOR IDENTIFIED NUCLIDES

THROUGH 12/31/04

CURIES REMAINING

$\begin{array}{rr}3 & 53 \% \\ <1 & 97 \% \\ 81 & 97 \% \\ <1 & 100 \% \\ <1 & 100 \%\end{array}$

$84 \quad 94 \%$

DETAILS MAY NOT ADD UP TO TOTALS BECAUSE OF ROUNDING

WMC 1985-1994 SOLID DISPOSED WASTE SUMMARY

IN CURIES, DECAYED VALUE FOR IDENTIFIED NUCLIDES

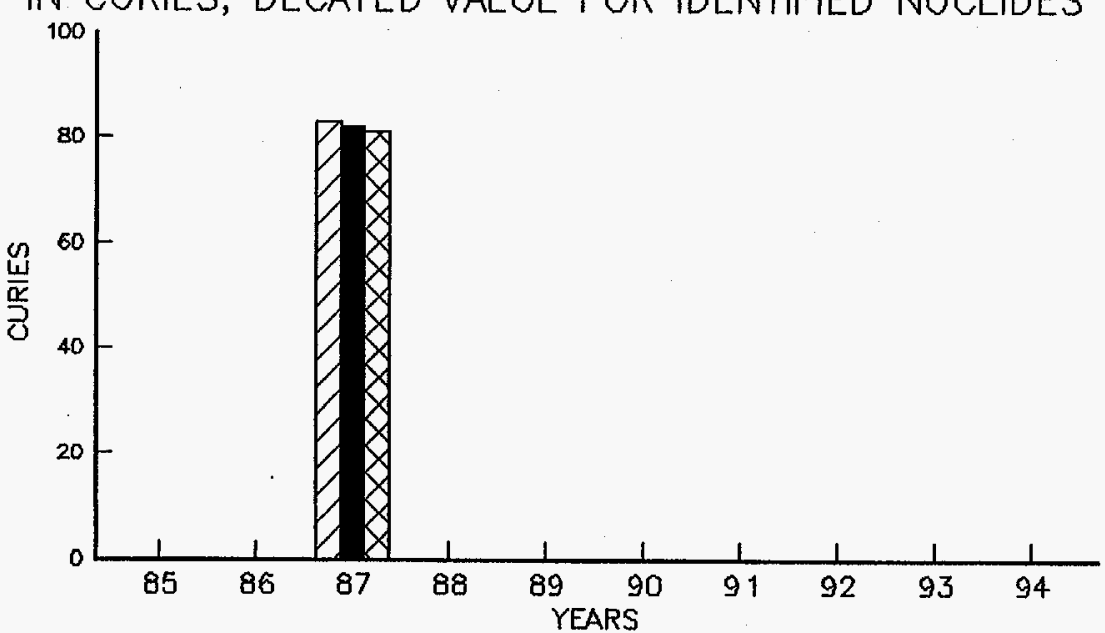

UDISPOSED $\square$ DECAYED ON $12 / 31 / 94 \square D E C A Y E D$ ON $12 / 31 / 04$ 
INEL 1994 Year-to-Date Summary

INEL Year To Date Summary, $1994 \ldots \ldots \ldots \ldots \ldots \ldots \ldots \ldots \ldots$ INEL-105

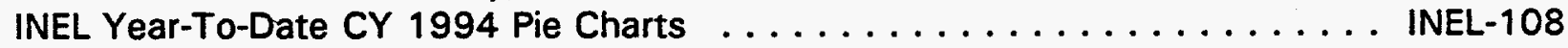

INEL Year-To-Date Summary CY 1994 Bar Graphs . . . . . . . . . . . . . INEL-109 

U.S. DEPARTMENT OF ENERGY

INEL WASTE MUNAGEMENT INFORMATION SYSTEM

INEL YEAR TO DATE SUMAARY $\quad$ PE I RPT. 2

FOR JANUARY THROUGH DECEMBER 1994

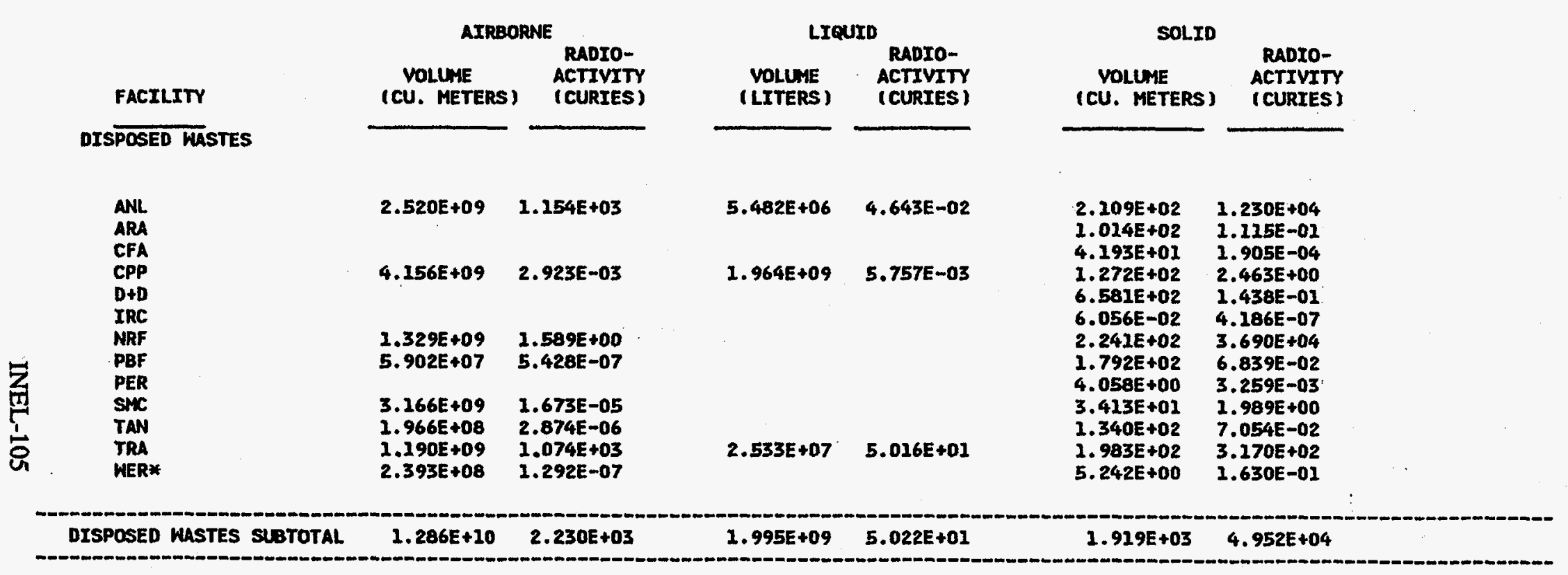

* solid values are for maste disposed at rHWC and are end products of werf reduction paocesses. 
SCHEDULE P6IPMO09-8

STORED WASTES

ANL STORAGE AREA

ANL

$0.000 E+00 \quad 0.000 E+00$

$0.000 E+00$

$0.000 E+00$

ILTSF WASTE FROM:

TRA
IDAHO OPERATIONS OFFICE

DEPARTMENT OF ENERGY

INEL WASTE MANAGEMENT INFORMATION SYSTEM

INEL YEAR TO DATE SUMYARY

FOR JAMUARY THROUGH DECEMBER 1994

AIRBORNE

VOLIME RADTO

(CU. METERS) (CURIES)

\section{LIQUID}

(LITERS) ACTIVITY

(CURIES)

(CU. METERS

SOLID

RADIO-

ACTIVITY

PG 2 RPT.

\section{章}

$0.000 E+00 \quad 0.000 E+00$

$0.000 E+00 \quad 0.000 E+00$

5.000E-01 7.261E+04

5.000E-01 7.261E+04

5.177E+00 5.313E+01

5. $177 E+00 \cdot 5.313 E+01$

STORED WASTES SUBTOTAL


U.S. DEPARTMENT OF ENERGY

INEL WASTE MANAGEMENT INFORMATION SYSTEM

INEL YEAR TO DATE SUMMARY

FOR JAMUARY THROUGH DECEMBER 199

\begin{tabular}{|c|c|c|c|c|c|c|}
\hline \multirow[b]{2}{*}{ FACILITY } & \multicolumn{2}{|c|}{ AIRBORNE } & \multicolumn{2}{|c|}{ LIEUID } & \multicolumn{2}{|l|}{ SOLID } \\
\hline & $\begin{array}{l}\text { VOLUME } \\
\text { (CU. METERS ) }\end{array}$ & $\begin{array}{l}\text { RADIO- } \\
\text { ACTIVITY } \\
\text { (CURIES) }\end{array}$ & $\begin{array}{l}\text { VOLUME } \\
\text { (LITERS) }\end{array}$ & $\begin{array}{l}\text { RADIO- } \\
\text { ACTIVITY } \\
\text { (CURIES) }\end{array}$ & $\begin{array}{l}\text { VOLUNE } \\
\text { (CU. METERS) }\end{array}$ & $\begin{array}{l}\text { RADIO- } \\
\text { ACTIVITY } \\
\text { (CURIES ) }\end{array}$ \\
\hline $\begin{array}{l}\text { WASTES TO BE } \\
\text { AT WERF FR }\end{array}$ & & & & & & \\
\hline $\begin{array}{l}C F A \\
D+D \\
N R F \\
\text { SMC }\end{array}$ & & & & & $\begin{array}{l}9.140 E+01 \\
1.146 E+02 \\
2.832 E+02 \\
2.802 E+02\end{array}$ & $\begin{array}{l}2.650 E-03 \\
2.714 E-03 \\
1.357 E+00 \\
6.060 E-03\end{array}$ \\
\hline
\end{tabular}




\section{INEL Year-to-Date Summary CY 1994}

INEL AIRBORNE EFFLUENT

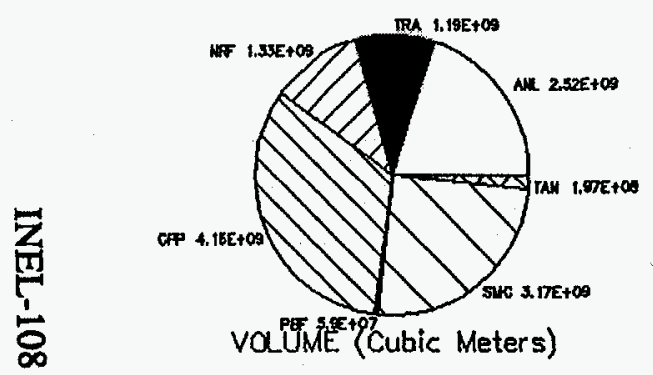

INEL LIQUID EFFLUENT
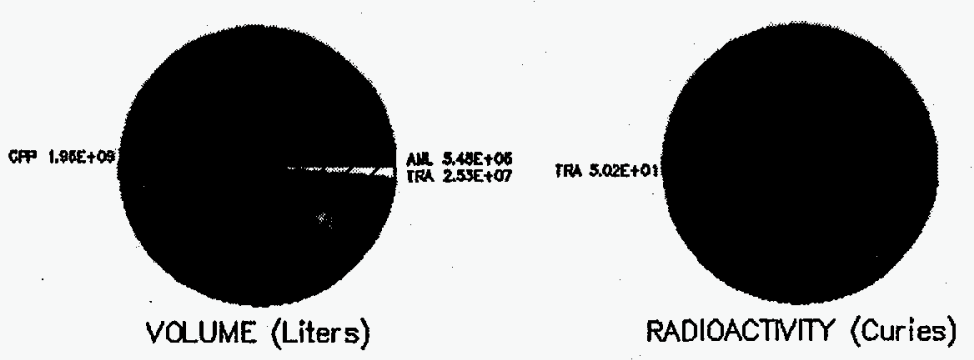

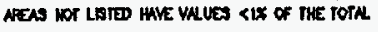

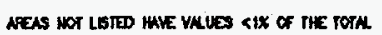

INEL SOLID WASTE VOLUME (Cubic Meters) DISPOSED AT RWMC AND SENT FOR REDUCTION EFFORTS

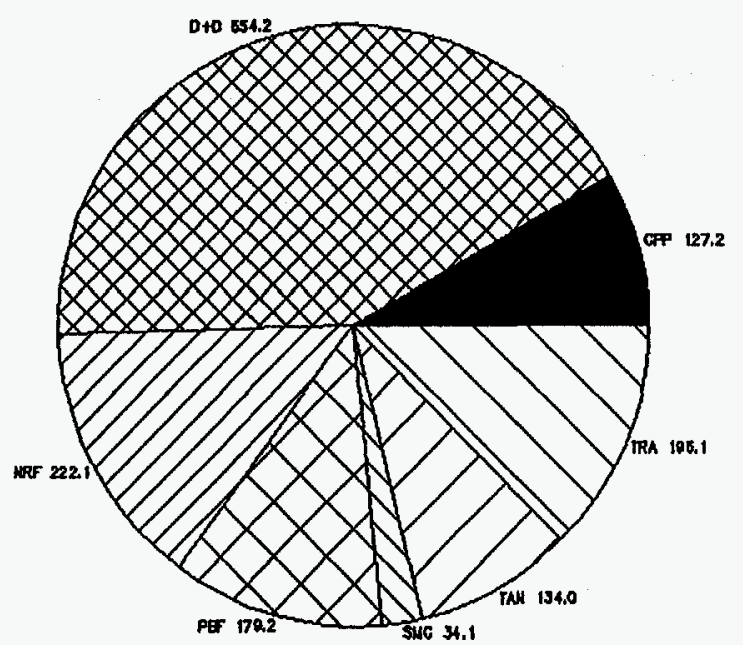

NREAS HOY LISED HAVE VNLUES \&LX OF THE TOYA 


\section{INEL Year-to-Date Summary CY 1994}
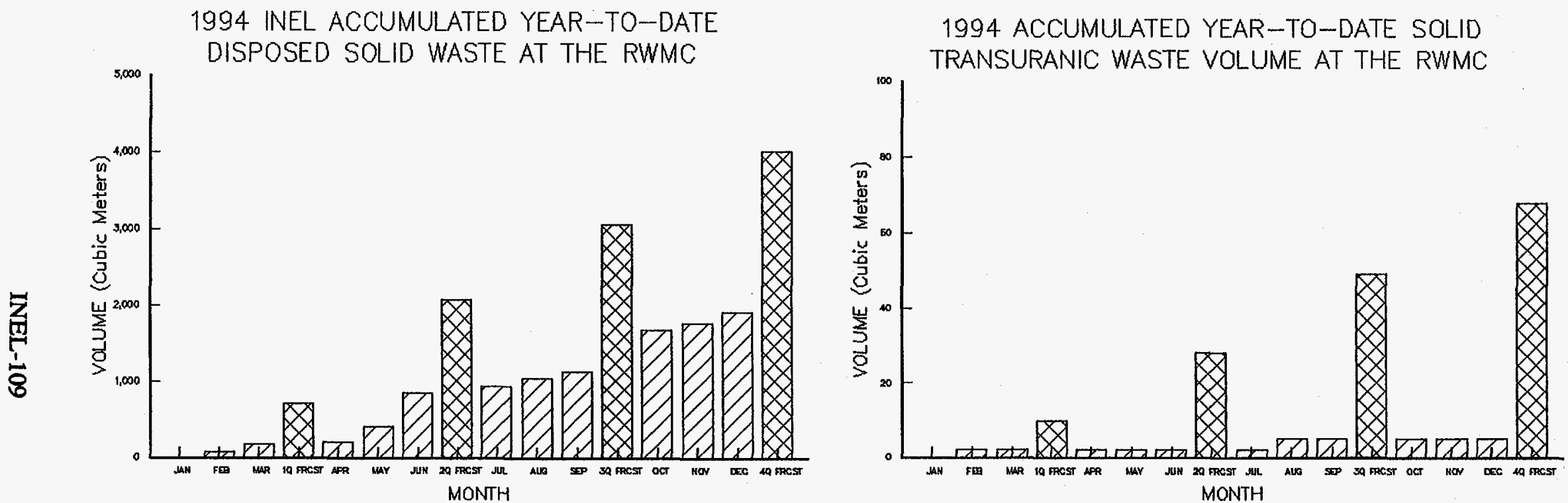

DACTUAL $\triangle F O R E C A S T$

DACTUAL GFORECAST

1994 INEL ACCUMULATED YEAR-TO-DATE SOLID LOW LEVEL WASTE

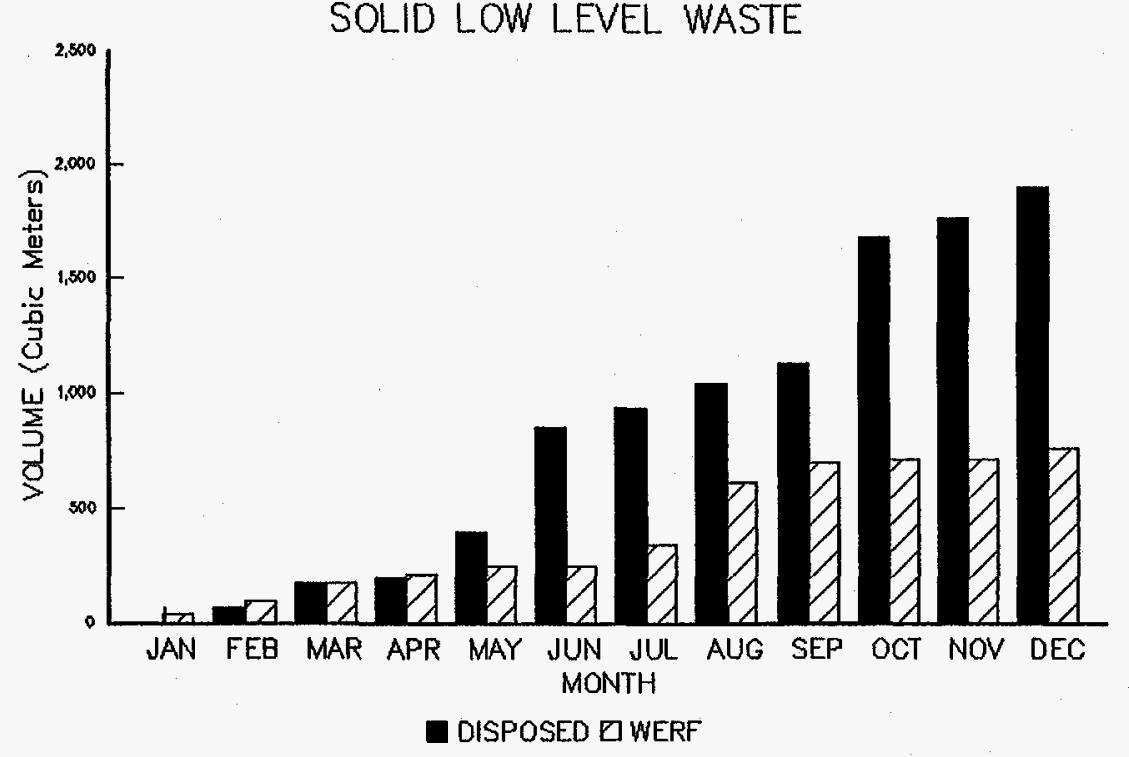




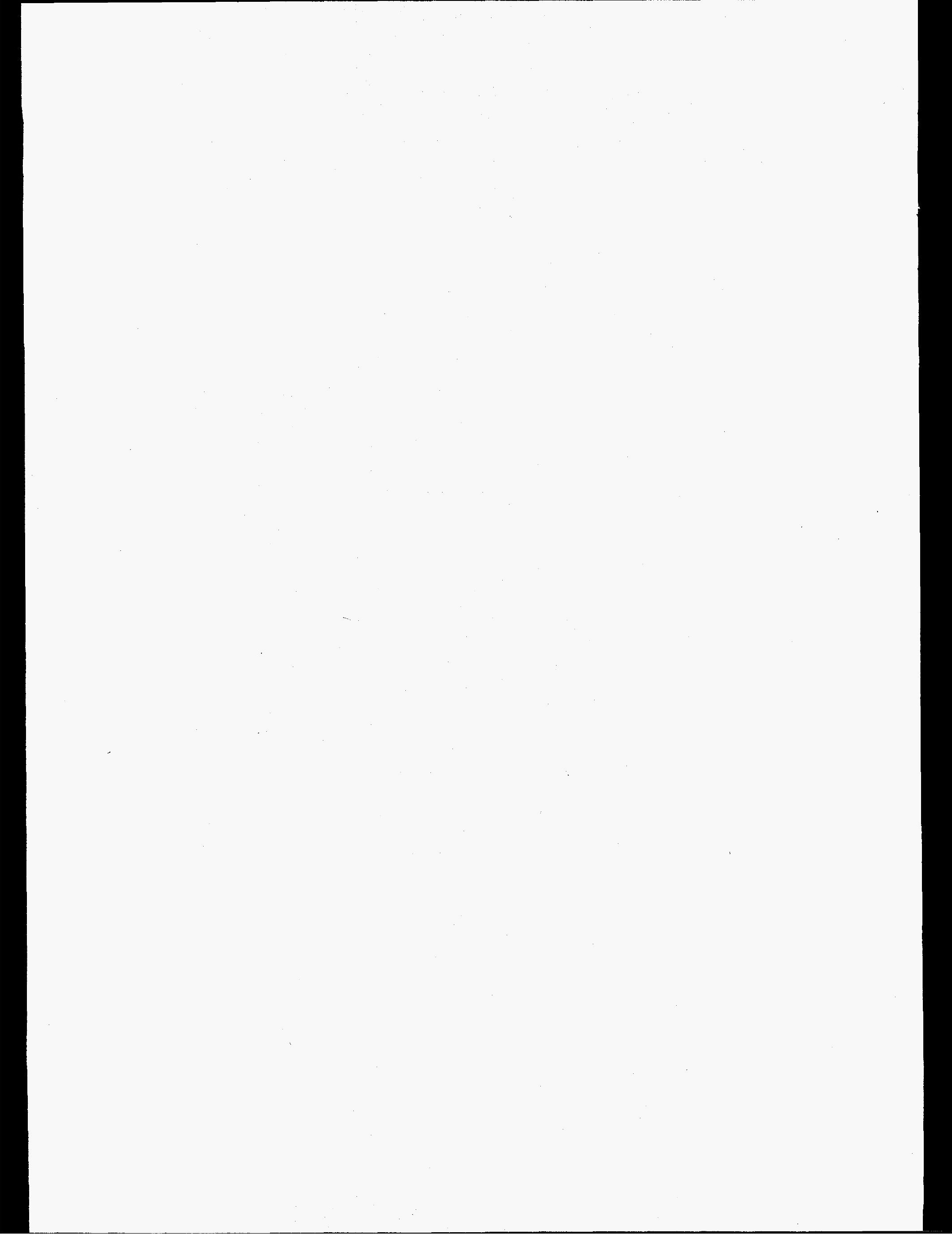


INEL Disposed Waste Decay Summary in Curies for 1994

INEL Disposed Waste Decay Summary in Curies for $1994 \ldots \ldots \ldots \ldots$ INEL-113 


\begin{tabular}{|c|c|c|c|c|c|c|c|c|c|}
\hline & & DISPC & ECAY SUMA & RY IN CURIE & ES FOR 1994 & & & & \\
\hline & $\begin{array}{l}\text { TOTAL } \\
\text { IDENTIFIED } \\
\text { NUCLIDES } \\
\text { AT TIME OF } \\
\text { DISPOSAL }\end{array}$ & $\begin{array}{l}\text { TOTAL } \\
\text { UNIDENTIFIED } \\
\text { NUCLIDES } \\
\text { AT TIME OF } \\
\text { DISPOSAL }\end{array}$ & $01 / 01 / 95$ & $\begin{array}{l}\text { DECAYED } \\
01 / 01 / 96\end{array}$ & $\begin{array}{l}\text { VALUE FOR } \\
01 / 01 / 97\end{array}$ & $\begin{array}{l}\text { IDENTIFIED } \\
01 / 01 / 98\end{array}$ & $\begin{array}{l}\text { MUCLIDES } \\
01 / 01 / 99\end{array}$ & $\begin{array}{l}\text { THROUGH - } \\
\text { O1/01/00 }\end{array}$ & $01 / 01 / 05$ \\
\hline AIRBORNE & 2230 & 0 & 49 & 46 & 44 & 42 & 40 & 38 & 31 \\
\hline LIQUID & 50 & o & 47 & 44 & 41 & 39 & 37 & 35 & 26 \\
\hline SOLID & 49524 & o & 44707 & 40660 & 37549 & 35047 & 32970 & 31215 & 25451 \\
\hline
\end{tabular}




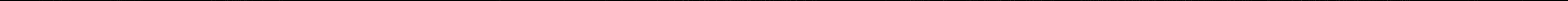


Nuclide Summary in Curies for Airborne, Liquid, and Solid Waste

Airborne Nuclide Summaries in Curies, $1994 \ldots \ldots \ldots \ldots \ldots \ldots \ldots$ INEL-117

Liquid Nuclide Summary in Curies, $1994 \ldots \ldots \ldots \ldots \ldots \ldots \ldots \ldots \ldots$ INEL-119

Disposed Solid Waste Nuclide Summary in Curies, $1994 \ldots \ldots \ldots \ldots \ldots \ldots$ INEL-120

ILTS Area Nuclide Summary in Curies, $1994 \ldots \ldots \ldots \ldots \ldots \ldots \ldots \ldots \ldots$ INEL-122

ANL Storage Area Nuclide Summary in Curies, $1994 \ldots \ldots \ldots \ldots \ldots \ldots \ldots$. . . . . . . . . 23

WERF Processed Nuclide Summary in Curies, $1994 \ldots \ldots \ldots \ldots \ldots$. . . . . INEL-124 

INEL WASTE MANAGEMENT INFORMATION SYSTEM

$$
\text { AIRBORNE }
$$

MUCLIDE SUMMARY IN CURIES

FOR JANUARY THROUGH DECEMBER 1994

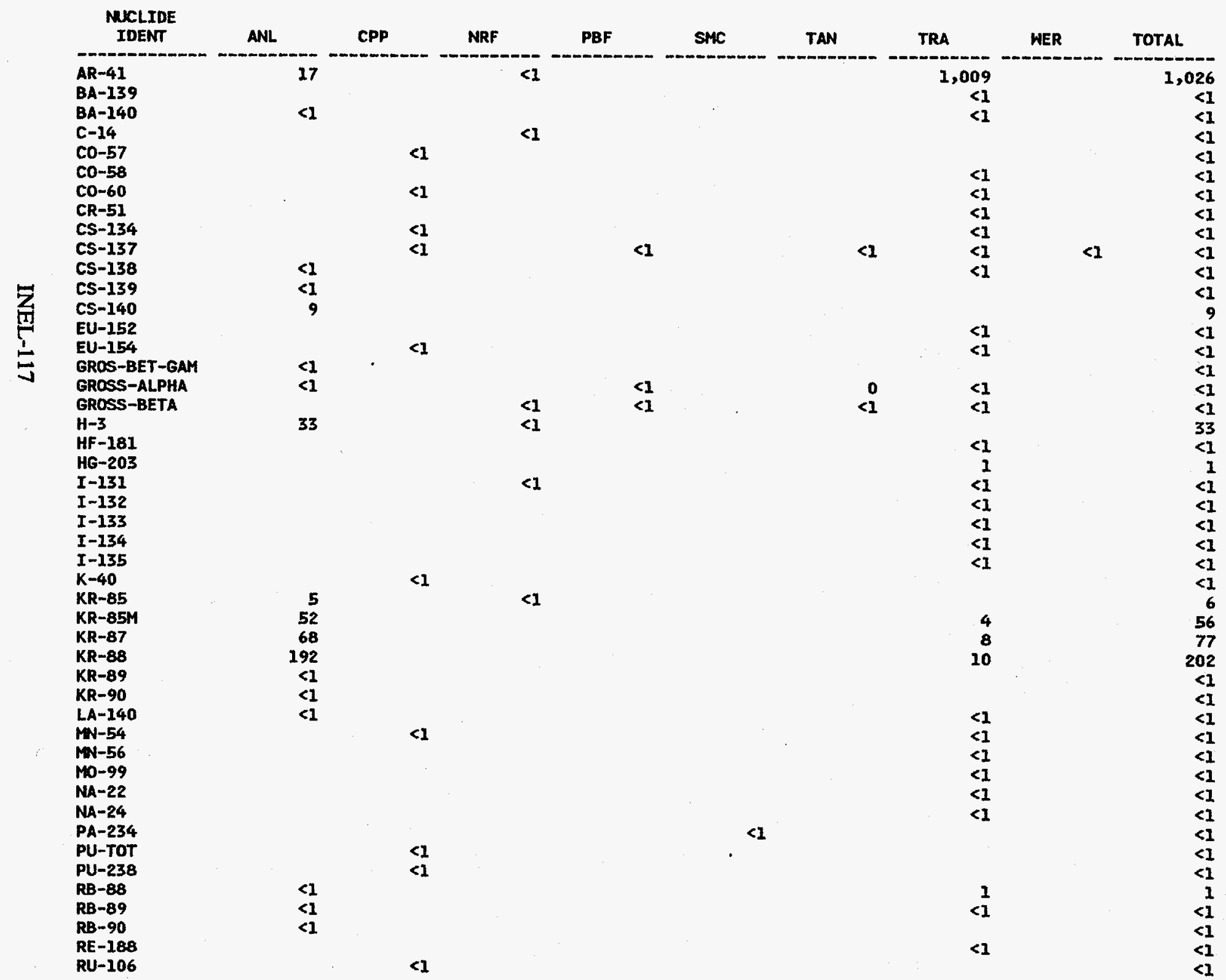


INEL WASTE MANAGEMENT INFORMATION SYSTEM

$$
\text { AIRBORNE }
$$

MUCLIDE SUMARY IN CURIES

FOR JANUARY THROUGH DECEMBER 1994

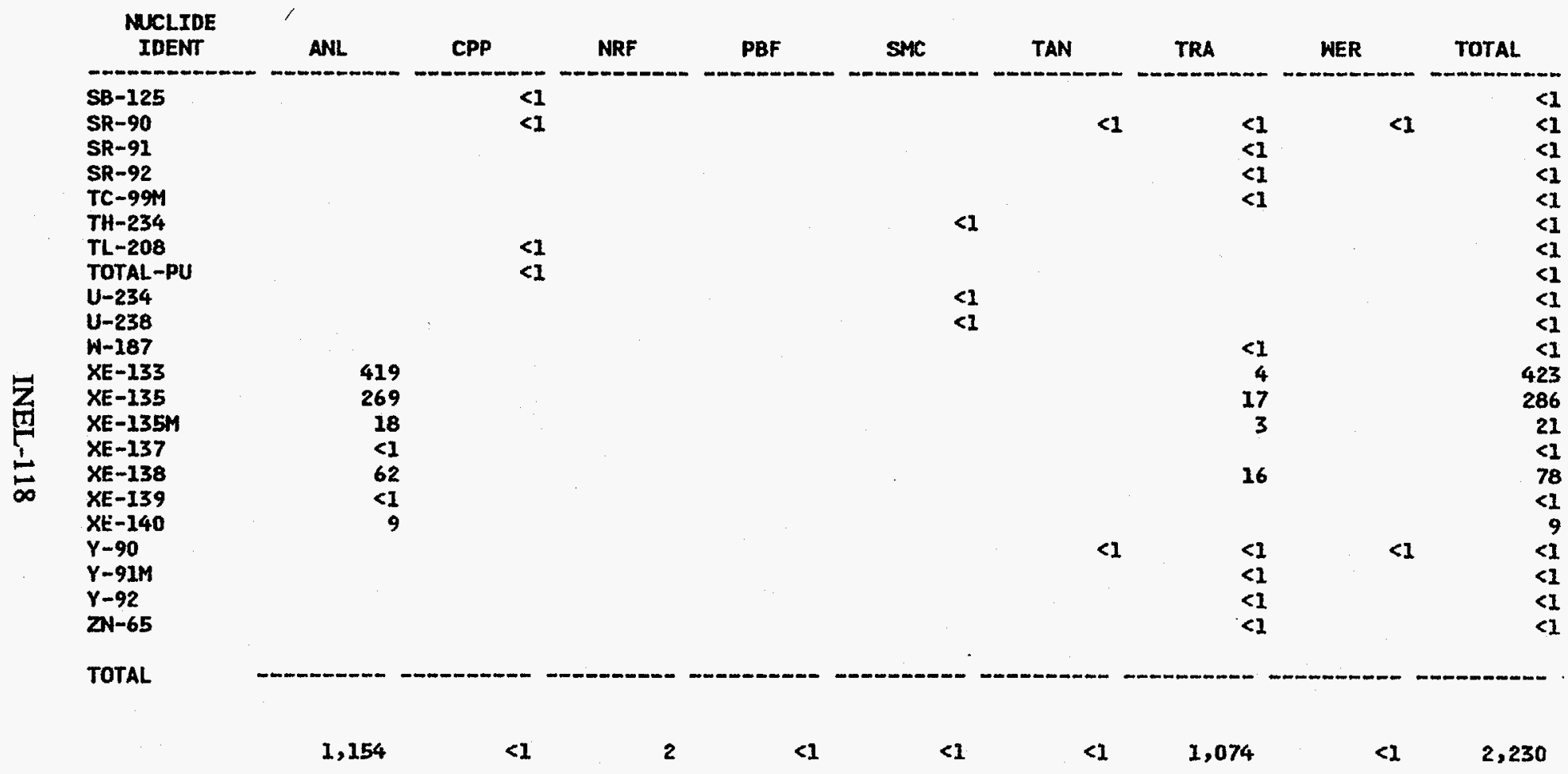


INEL WASTE MANAGEMENT INFORMATION SYSTEM

IN CURIES

FOR JANUARY THROUGH DECEMBER 1994

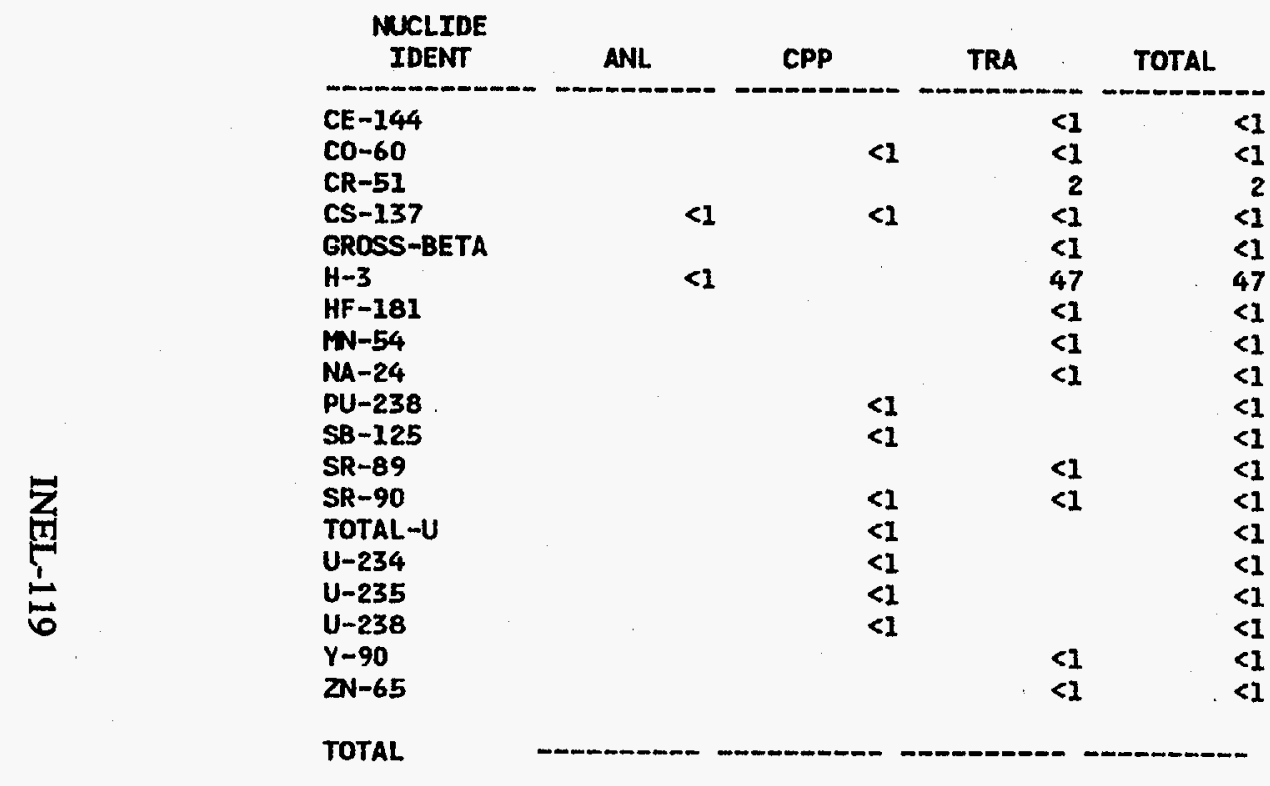

$<1$

$<1$

50

50 
INEL WASTE MANAGEMENT INFORMATION SYSTEM

\section{DISPOSED SOLID NASTE}

MUCLTDE SUMARY IN CURTES

PG 1

FOR JAMARY THROUGH DECEMBER 1994

\begin{tabular}{|c|c|c|c|c|c|c|c|c|c|c|c|c|c|c|}
\hline $\begin{array}{l}\text { MUCLIDE } \\
\text { IDENT }\end{array}$ & ANL & ARA & CFA & CPP & $D+D$ & IRC & NRF & PBF & PER & SMC & TAN & TRA & WER & TOTAL \\
\hline$A G-108 M$ & & & & & $<1$ & & & & & & $<1$ & & & $<1$ \\
\hline $\begin{array}{l}A G-110 \\
A G-110 Y\end{array}$ & & & & & & & & & & & & $<1$ & $<1$ & $\begin{array}{l}<1 \\
<1\end{array}$ \\
\hline $\begin{array}{l}A G-110 M \\
A M-241\end{array}$ & $<1$ & & $<1$ & & & & $<1$ & & & & $<1$ & $<1$ & $\begin{array}{l}<1 \\
<1\end{array}$ & \\
\hline$B A-13 M$ & $\therefore$ & & & & & & $<1$ & & & & & & $<2$ & $<1$ \\
\hline$B A-140$ & & & & & & & $<1$ & & & & & & & $<1$ \\
\hline$c-14$ & & & & & & & 1 & & & & & $<1$ & $<1$ & 1 \\
\hline CE-141 & $<1$ & & & & & & & & & & & $<1$ & & $<1$ \\
\hline CE-144 & $<1$ & & $<1$ & & & & $<1$ & & & & & $<1$ & $<1$ & $<1$ \\
\hline$C M-242$ & & & & & & & & & & & & & $<1$ & $<1$ \\
\hline CM-244 & & & $<1$ & & & & & & & & $<1$ & & $<1$ & $<1$ \\
\hline Co-57 & & & & & & & & & & & & & $<1$ & $<1$ \\
\hline CO-58 & 460 & & $<1$ & & & & 9 & & & & & $<1$ & & 470 \\
\hline co-60 & 10,148 & & $<1$ & $<1$ & $<1$ & & 7,416 & & $<1$ & & $<1$ & 172 & $<1$ & 17,737 \\
\hline$C R-51$ & 1 & & & & & & $<1$ & & & & & 33 & & 34 \\
\hline CS-134 & $<1$ & & $<1$ & & & & & & $<1$ & & $<1$ & $<1$ & $<1$ & $<1$ \\
\hline CS- 137 & 1 & $<1$ & $<1$ & 2 & $<1$ & & $<1$ & $<1$ & $<1$ & & $<1$ & 2 & $<1$ & 5 \\
\hline EU-152 & & & $<1$ & $<1$ & $<1$ & & $<1$ & & & & $<1$ & $<1$ & $<1$ & $<1$ \\
\hline EU-154 & $<1$ & & $<1$ & $<1$ & & & $<1$ & & $<1$ & & $<1$. & $<1$ & $<1$ & $<1$ \\
\hline EU-155 & $<1$ & & $<1$ & & & & $<1$ & & & & $<I$ & $<1$ & $<1$ & $<1$ \\
\hline FE- 55 & & & & & & & 5,790 & & & & & 28 & $<I$ & 5,818 \\
\hline FE-59 & 2 & & & & & & $<1$ & & & & & $<1$ & & 2 \\
\hline $\mathrm{H}-3$ & $<1$ & & & & & & $<1$ & & & & & $<1$ & & $<1$ \\
\hline$H F-181$ & & & & & & & $<1$ & & & & & $<1$ & & $<1$ \\
\hline $1-129$ & $<1$ & & & & & & $<1$ & & & & & & & $<1$ \\
\hline$I-131$ & & & & & & & & & & & & $<1$ & & $<1$ \\
\hline IR-192 & & & & & & & & & & & & $<1$ & & $<1$ \\
\hline$K-40$ & & & & & & & & & $<1$ & & $<1$ & & & $<1$ \\
\hline$K R-85$ & & & & & & & $<1$ & & & & & & & $<1$ \\
\hline$L A-140$ & & & & & & & & & & & & $<1$ & & $<1$ \\
\hline$M N-54$ & 1,682 & & $<1$ & & & & $<1$ & & $<1$ & & $<1$ & 3 & $<1$ & 1,685 \\
\hline MO-99 & & & & & & & & & & & & $<1$ & & $<1$ \\
\hline NA-22 & $<1$ & & & & & & & & $<1$ & & & & & $<1$ \\
\hline$N 8-94$ & & & & & & & $<1$ & & & & & $<1$ & & $<1$ \\
\hline NB-95 & $<1$ & & $<1$ & & & & 38 & & & & & $<1$ & & 38 \\
\hline NI -59 & & & & & & & 169 & & & & & $<1$ & & 169 \\
\hline$N I-63$ & & & & & & & 22,190 & & & & & $<1$ & $<1$ & 22,190 \\
\hline NP-237 & $<1$ & & $<1$ & & & & & & & & & & & $<1$ \\
\hline PA-234 & & & & & & $<1$ & & & & & & & $<1$ & $<1$ \\
\hline$P A-234 M$ & & & & & & & & & & & & & $<1$ & $<1$ \\
\hline PB-212 & & & & & $<1$ & & & & & & & & & $<1$ \\
\hline PM-147 & & & & & & & $<1$ & & & & & & & $<1$ \\
\hline$P R-144$ & & & & & & & $<1$ & & & & & & $<1$ & $<1$ \\
\hline PU-238 & $<1$ & & $<1$ & $<1$ & & & $<1$ & & & & $<1$ & $<1$ & $<1$ & $<1$ \\
\hline PU-239 & $<1$ & & $<1$ & $<1$ & & $<1$ & $<1$ & & & & $<1$ & $<1$ & $<1$ & $<1$ \\
\hline PU-240 & $<1$ & & & & & & & & & & & & & $<1$ \\
\hline PU-24I & & & & & & & $<1$ & & & & & & & $<1$ \\
\hline
\end{tabular}


INEL WASTE MANAGEMENT INFORMATION SYSTEM

\section{DISPOSED SOLID WASTE}

NUCLIDE SUMAARY IN CURIES

FOR JAMUARY THROUGH DECEMBER 1994

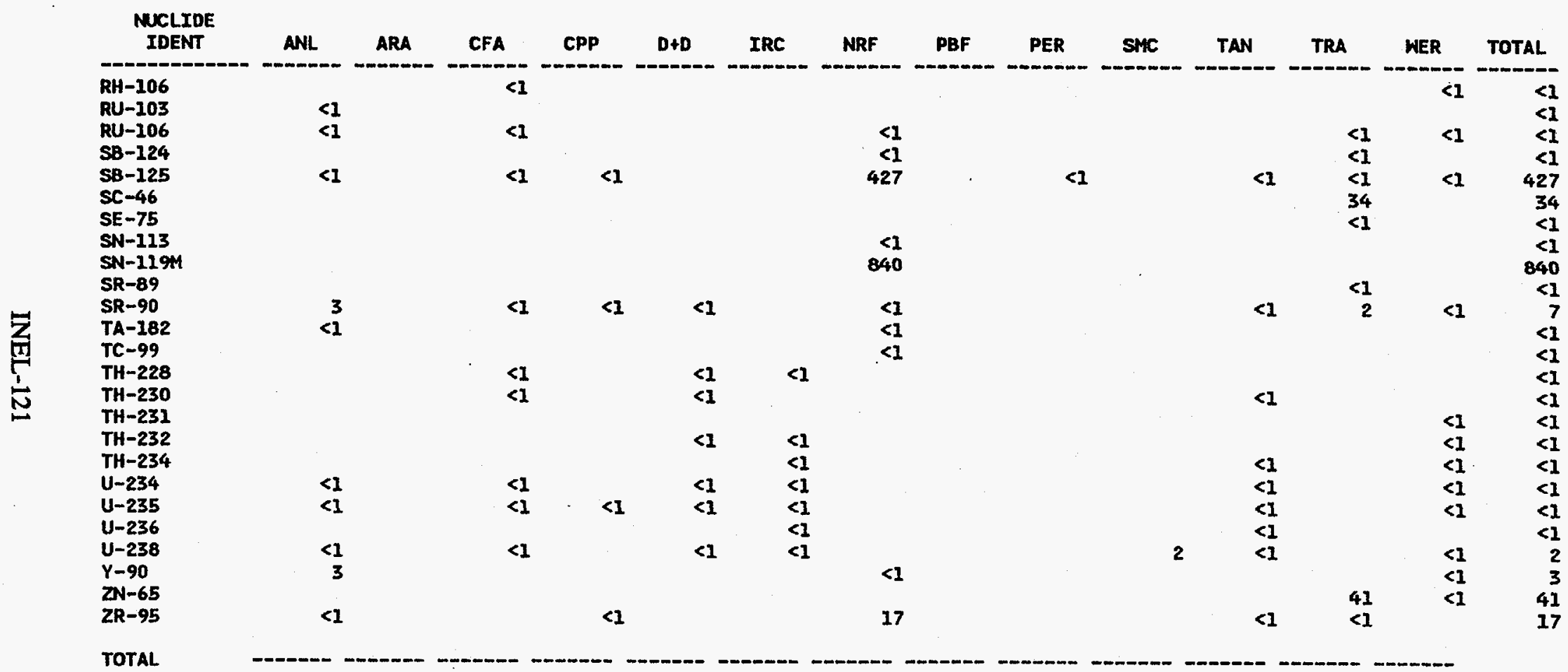

$\begin{array}{lllllllllllllll}12,301 & <1 & <1 & 2 & <1 & <1 & 36,901 & <1 & <1 & 2 & <1 & 317 & <1 & 49,524\end{array}$


INEL WASTE MANAGEMENT INFORMATION SYSTEM

INTERMEDIATE LEVEL TRANSURANIC STORAGE AREA

ICLIDE SUMARY IN CURIES

FOR JAMUARY THROUGH DECEMBER 1994

\begin{tabular}{lrr}
\multicolumn{1}{c}{$\begin{array}{c}\text { NUCLIDE } \\
\text { IDENT }\end{array}$} & TRA & TOTAL \\
\hline AM-241 & 23 & 23 \\
CM-242 & $<1$ & $<1$ \\
CM-244 & $<1$ & $<1$ \\
CO-60 & $<1$ & $<1$ \\
CR-51 & $<1$ & $<1$ \\
$C S-134$ & $<1$ & $<1$ \\
$C S-137$ & $<1$ & $<1$ \\
EU-152 & $<1$ & $<1$ \\
EU-154 & $<1$ & $<1$ \\
EU-155 & $<1$ & $<1$ \\
H-3 & $<1$ & $<1$ \\
MN-54 & $<1$ & $<1$ \\
PU-238 & 26 & 26 \\
$P U-239$ & $<1$ & $<1$ \\
SR-89 & $<1$ & $<1$ \\
SR-90 & 2 & 2 \\
$U-233$ & $<1$ & $<1$ \\
$U-234$ & $<1$ & $<1$ \\
$Z N-65$ & $<1$ & $<1$ \\
& &
\end{tabular}

TOTAL 
U.S. DEPARTMENT OF ENERGY

INEL MASTE MANAGEMENT INFORMATION SYSTEM

ANL STORAGE AREA

MUCLIDE SUMMARY IN CURIES

FOR JANUARY THROUGH DECEMBER 1994

\section{NUCLIDE}

IDENT

ANL.

TOTAL

AM-241

CE-144

CO-57

Co- 58

CO-60

CR-5I

CS -134

CS-137

EU-155

FE-59
$M N-54$

$\mathrm{MN}-54$
$\mathrm{PU}-239$

SB-125

SR-B5

$\mathrm{SR}-90$

$U-235$
$U-238$

$U-238$
$Y-90$

\begin{tabular}{rrr} 
ANL & TOTAL \\
$<1$ & $<1$ \\
$<1$ & $<1$ \\
$<1$ & $<1$ \\
3,960 & 3,960 \\
58,299 & 58,299 \\
622 & 622 \\
$<1$ & $<1$ \\
$<1$ & $<1$ \\
$<1$ & $<1$ \\
71 & 71 \\
9,658 & 9,658 \\
$<1$ & $<1$ \\
$<1$ & $<1$ \\
$<1$ & $<1$ \\
$<1$ & $<1$ \\
$<1$ & $<1$ \\
$<1$ & $<1$ \\
$<1$ & $<1$ \\
& \\
\hline & $<$
\end{tabular}

TOTAL

72,610

72,610 
INEL WASTE MANAGEMENT INFORMATION SYSTEM

WERF PROCESSED

MULITDE SUMARY IN CURIES

FOR JANUARY THROUGH DECEMBER 1994

\begin{tabular}{|c|c|c|c|c|c|}
\hline $\begin{array}{l}\text { MUCLIDE } \\
\text { IDENT }\end{array}$ & CFA & $D+D$ & NRF & SMC & TOTAL. \\
\hline$A G-110 M$ & & & $<1$ & & $<1$ \\
\hline AM-241 & & & $<1$ & & $<1$ \\
\hline$B A-137 M$ & & & $<1$ & & $<1$ \\
\hline$B A-140$ & & & $<1$ & & $<1$ \\
\hline$c-14$ & & & $<1$ & & $<1$ \\
\hline CE - 144 & $<1$ & & $<1$ & & $<1$ \\
\hline $\mathrm{CM}-242$ & & & $<1$ & & $<1$ \\
\hline CO-57 & & & $<1$ & & $<1$ \\
\hline CO-58 & & & $<1$ & & $<1$ \\
\hline co-60 & $<1$ & $<1$ & $<1$ & & $<1$ \\
\hline$C R-51$ & & & $<1$ & & $<1$ \\
\hline CS -134 & & & $<1$ & & $<1$ \\
\hline CS -137 & $<1$ & $<1$ & $<1$ & & $<1$ \\
\hline EU-152 & & & $<1$ & & $<1$ \\
\hline EU-154 & & & $<1$ & & $<1$ \\
\hline EU-155 & & & $<1$ & & $<1$ \\
\hline FE -55 & & & $<1$ & & $<1$ \\
\hline FE-59 & & & $<1$ & & $<1$ \\
\hline GD-153 & & & $<1$ & & $<1$ \\
\hline$H-3$ & & & $<1$ & & $<1$ \\
\hline$H F-175$ & & & $<1$ & & $<1$ \\
\hline$H F-181$ & & & $<1$ & & $<1$ \\
\hline$I-129$ & & & $<1$ & & $<1$ \\
\hline$K R-85$ & & & $<1$ & & $<1$ \\
\hline$M N-54$ & & & $<1$ & & $<1$ \\
\hline MO-99 & & & $<1$ & & $<1$ \\
\hline$N B-94$ & & & $<1$ & & $<1$ \\
\hline NB-95 & & & $<1$ & & $<1$ \\
\hline$N I-59$ & & & $<1$ & & $<1$ \\
\hline$N I-63$ & & & $<1$ & & $<1$ \\
\hline PM-147 & & & $<1$ & & $<1$ \\
\hline PR-144 & & & $<1$ & & $<1$ \\
\hline PU-238 & & & $<1$ & & $<1$ \\
\hline PU-239 & & & $<1$ & & $<1$ \\
\hline PU-241 & & & $<1$ & & $<1$ \\
\hline RH-106 & & & $<1$ & & $<1$ \\
\hline RU-106 & & & $<1$ & & $<1$ \\
\hline $58-124$ & & & $<1$ & & $<1$ \\
\hline$S B-125$ & & & $<1$ & & $<1$ \\
\hline SM-151 & & & $<1$ & & $<1$ \\
\hline SN-II3 & & & $<1$ & & $<1$ \\
\hline SR-90 & $<1$ & $<1$ & $<1$ & & $<1$ \\
\hline TA-182 & & & $<1$ & & $<1$ \\
\hline TC-99 & & & $<1$ & & $<1$ \\
\hline$U-238$ & $<1$ & & & $<1$ & $<1$ \\
\hline$Y-90$ & & & $<1$ & & $<1$ \\
\hline$Z R-95$ & & & $<1$ & & $<1$ \\
\hline
\end{tabular}


U.S. DEPARTIYENT OF ENERRGY

INEL MASTE MANAGEMENT INFORMATION SYSTEM

\section{WERF PROCESSED}

NUCLIDE SUTEARY IN CURIES

MCLIDE

IDENT

CFA

$D+D$

NRF

SMC

\section{TOTAL}

TOTAL 

Argonne National Laboratory-West 1994 Detail Data

Argonne National Laboratory-West (ANL) Bar Graphs

of Annual Data by Month . . . . . . . . . . . . . . . . . . INEL-129 



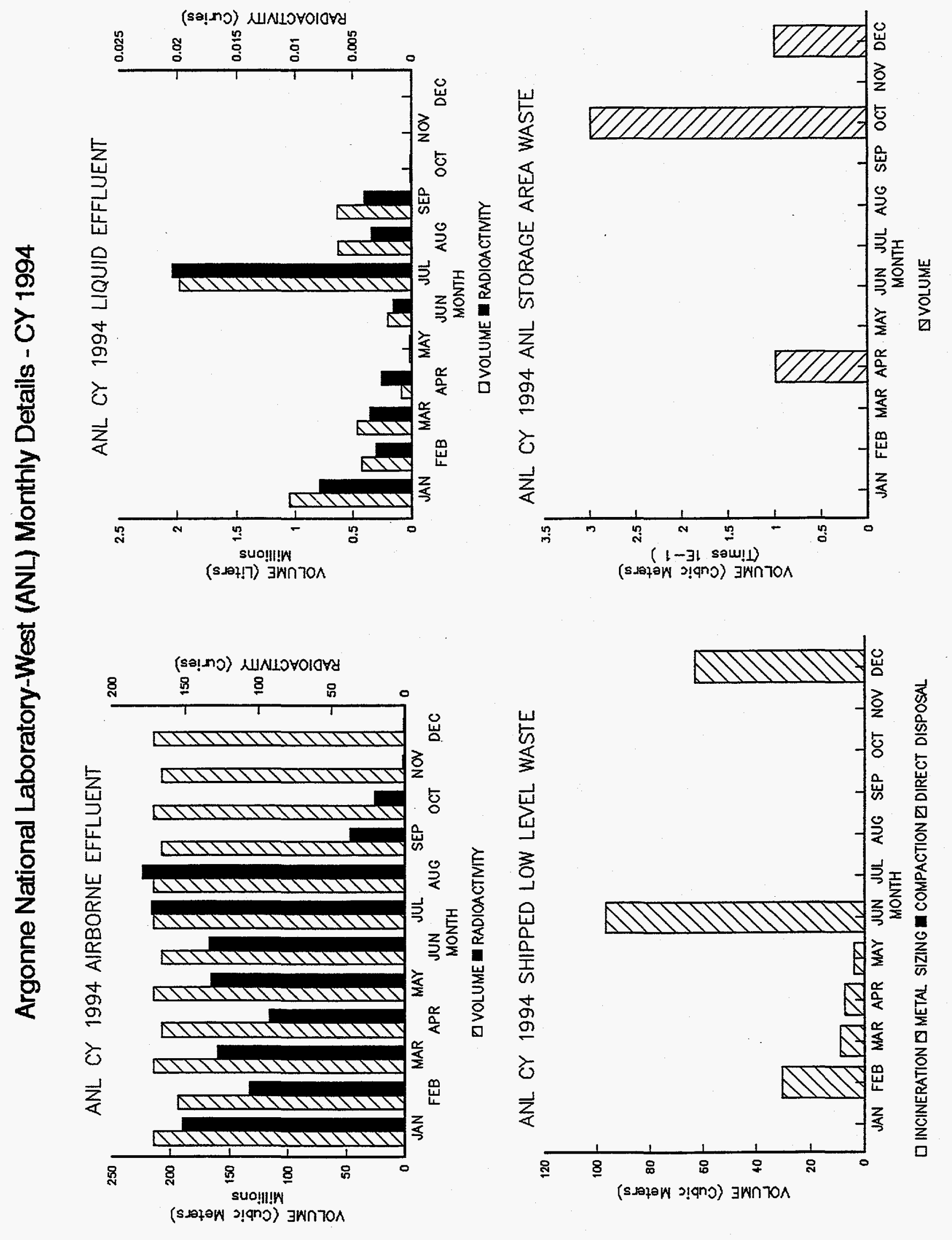

INEL-129 


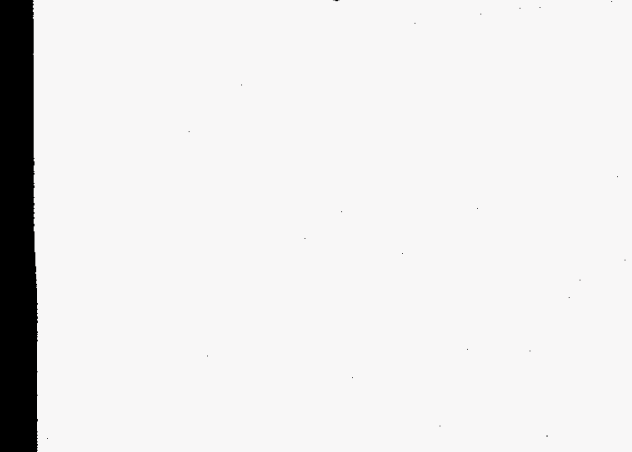


Central Facilities Area 1994 Detail Data

Central Facilities Area (CFA) Bar Graphs of Annual Data by Month $\ldots \ldots \ldots$ INEL-133 


\section{Central Facility Area (CFA) Monthly Details - CY 1994}

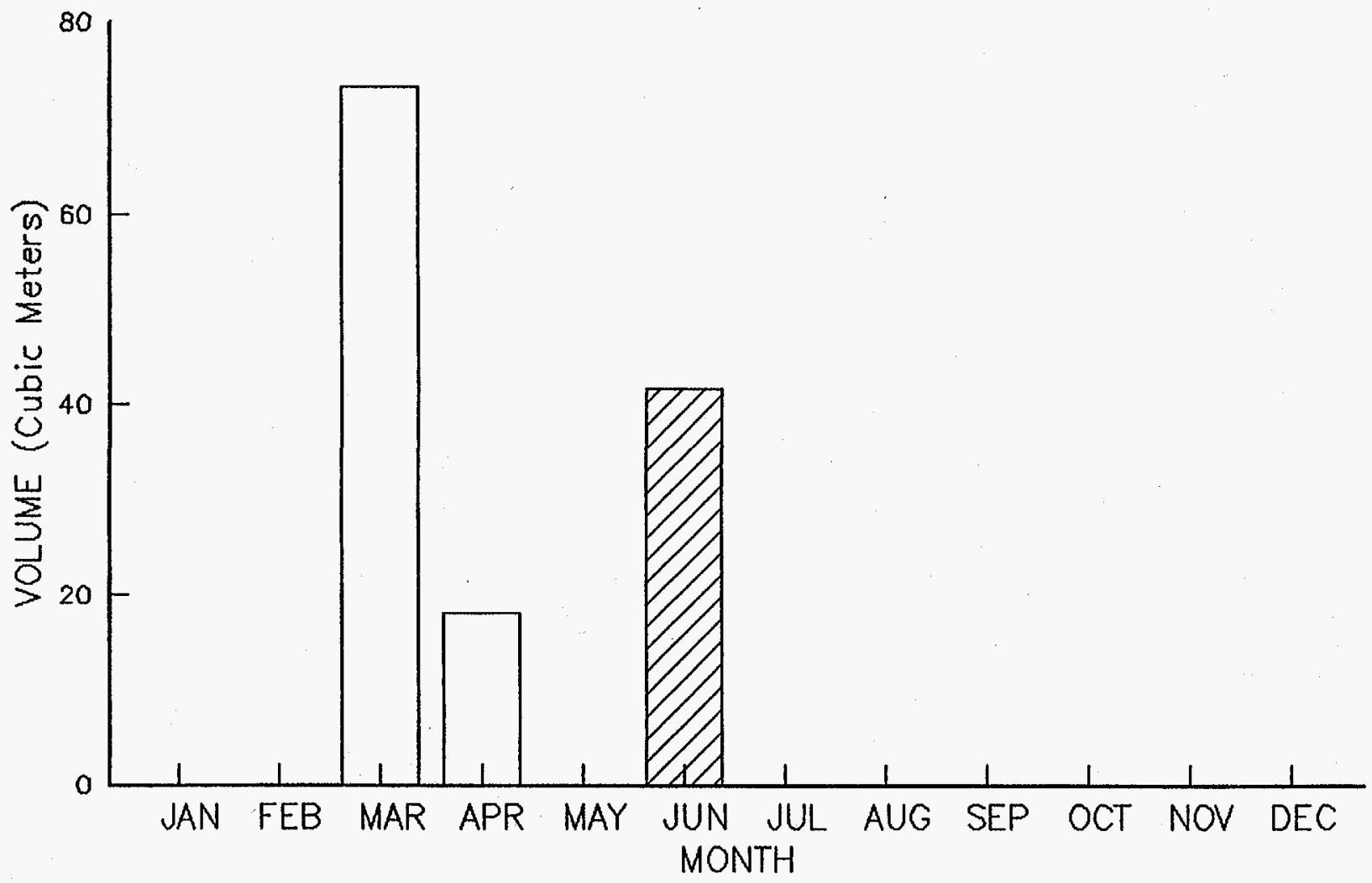

$\square$ INCINERATION METAL SIZING COMPACTION $\square$ DIRECT DISPOSAL 


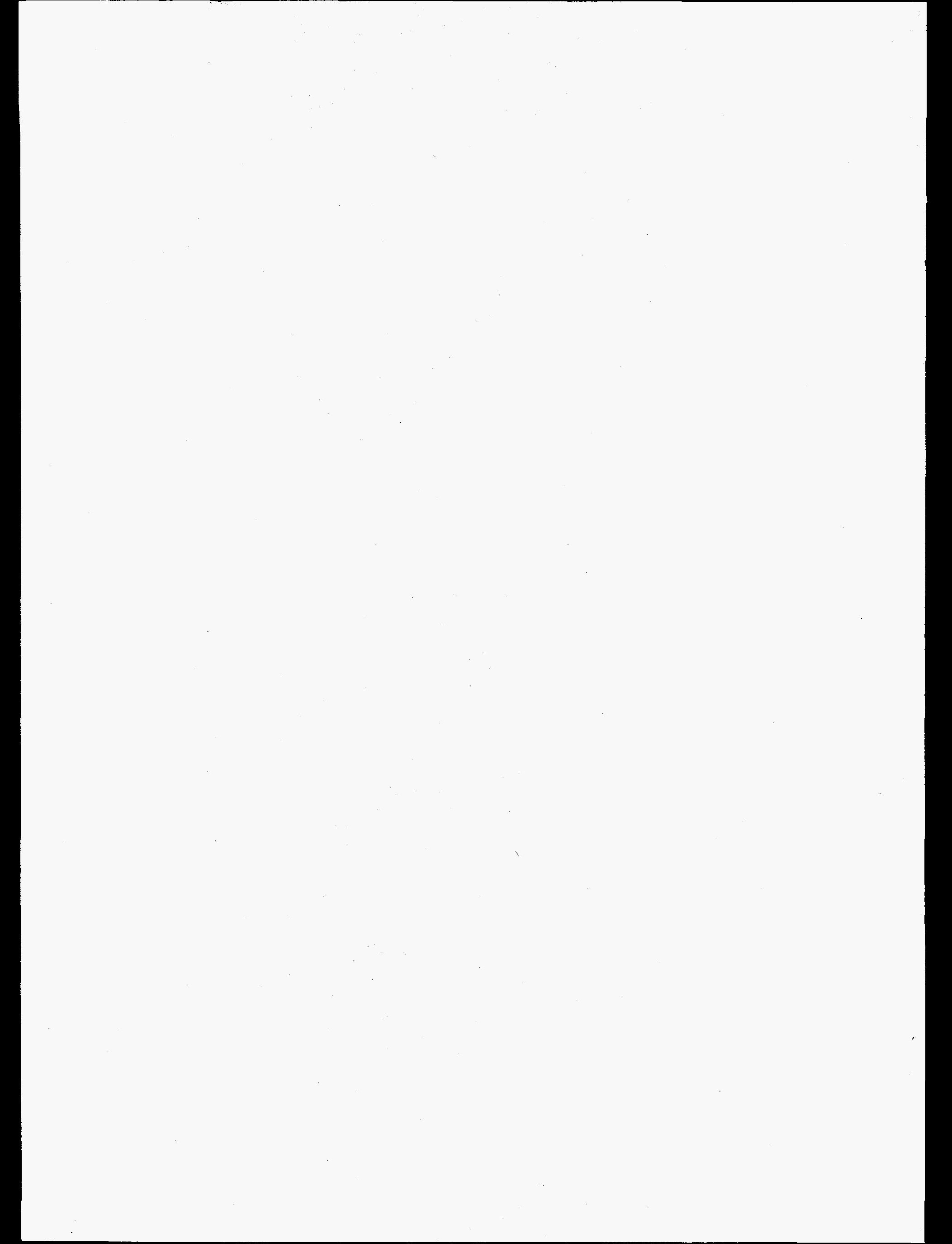


Chemical Processing Plant 1994 Detail Data

Chemical Processing Plant (CPP) Bar Graphs of Annual Data by Month $\ldots .$. INEL-137 



\section{Chemical Processing Plant (CPP) Monthly Details - CY 1994}
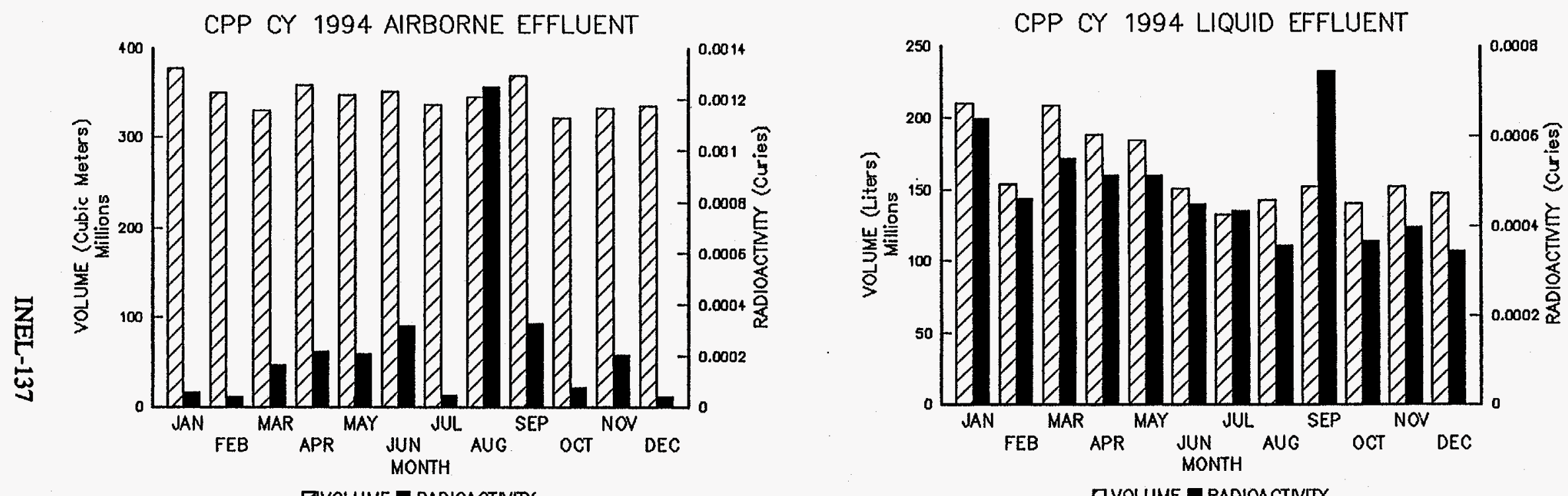

ZUVOLUME I RADIOACTNTT

DVOLUME Q RADIOACTNITY

CPP CY 1994 SHIPPED LOW LEVEL WASTE

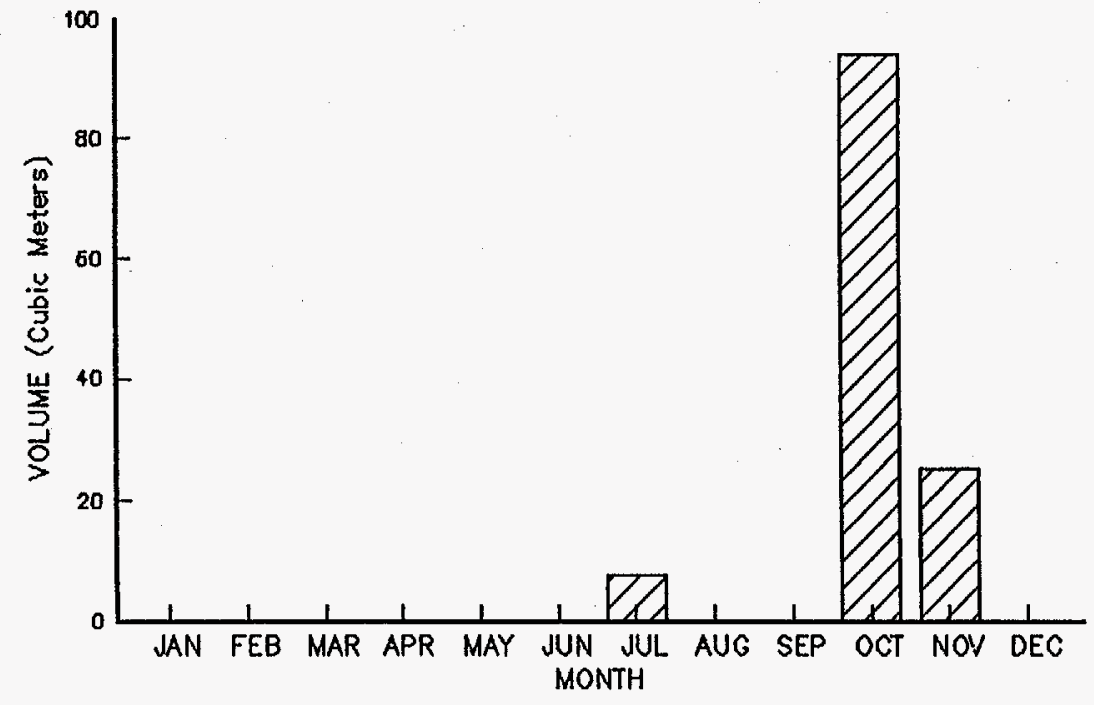

DINCINERATION $\triangle$ METAL SIZING $\approx$ COMPACTION $\square$ DIRECT DISPOSAL

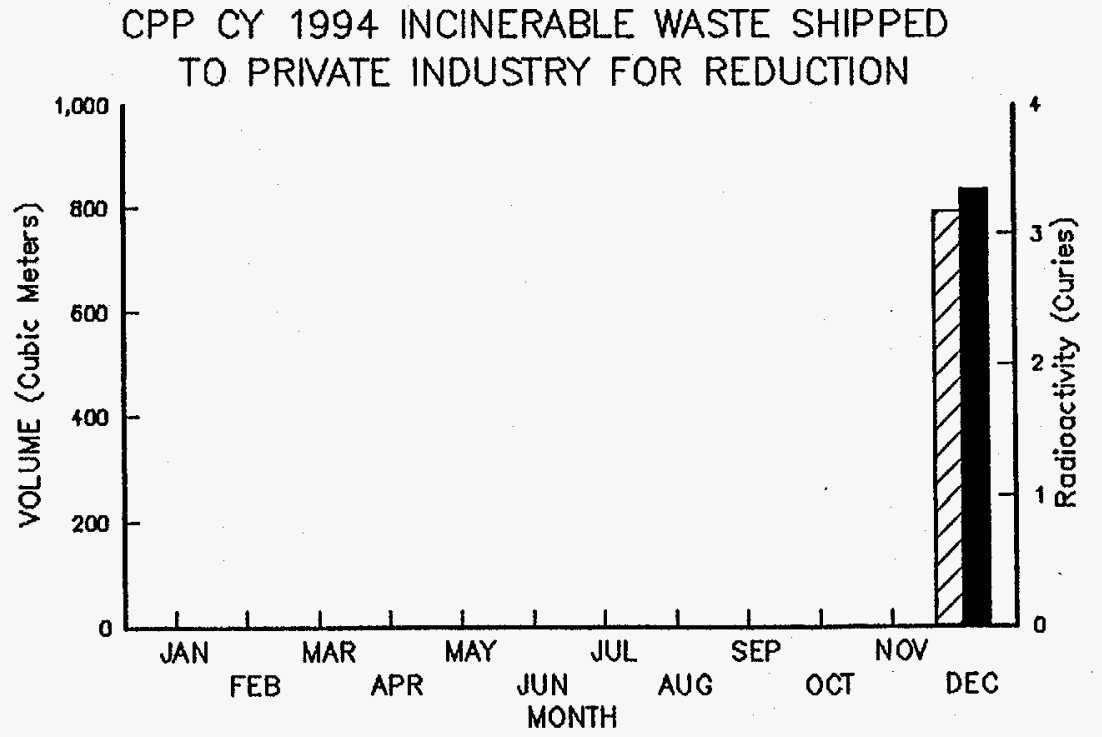

EVOLUME DCURIES 


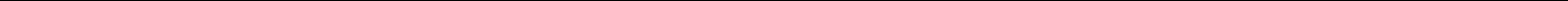


Decontamination and Decommissioning 1994 Detail Data

Decontamination and Decommissioning (D\&D) Bar Graphs

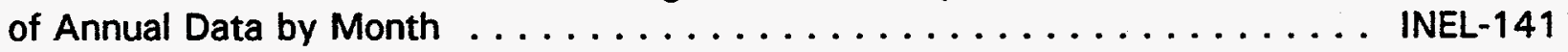





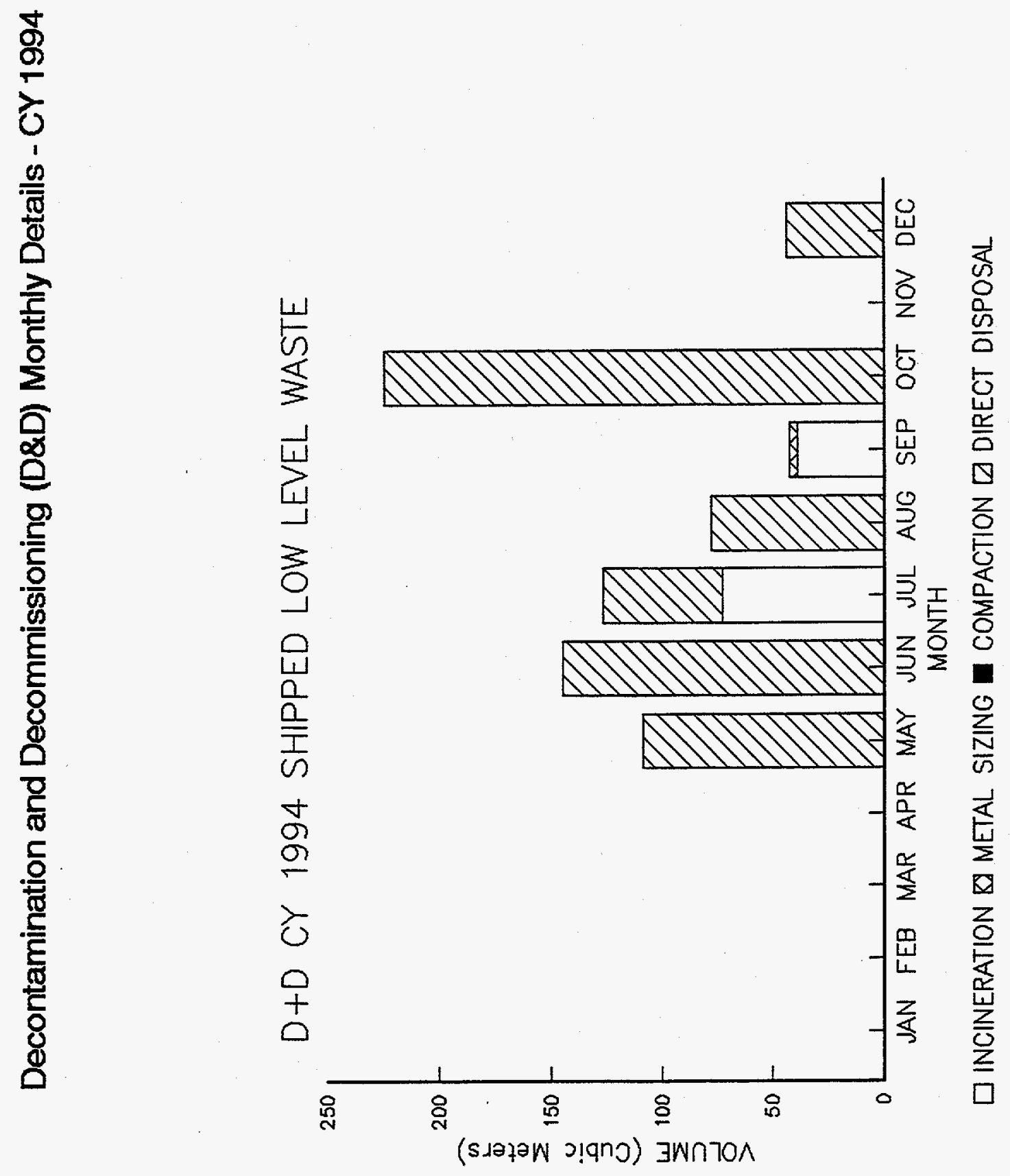



Naval Reactor Facility 1994 Detail Data

Naval Reactor Facility (NRF) Bar Graphs of Annual Data by Month . . . . . . . INEL-145 



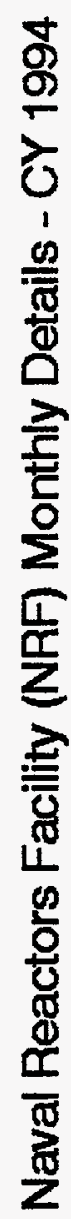
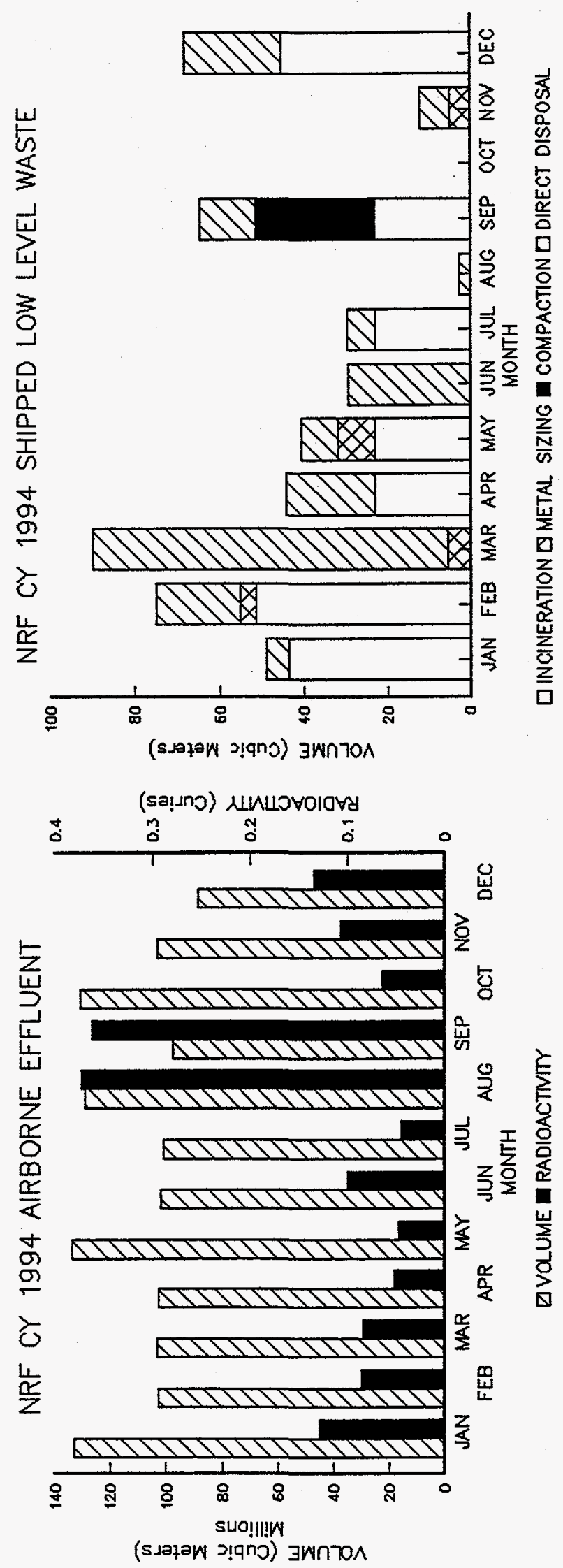

INEL-145 


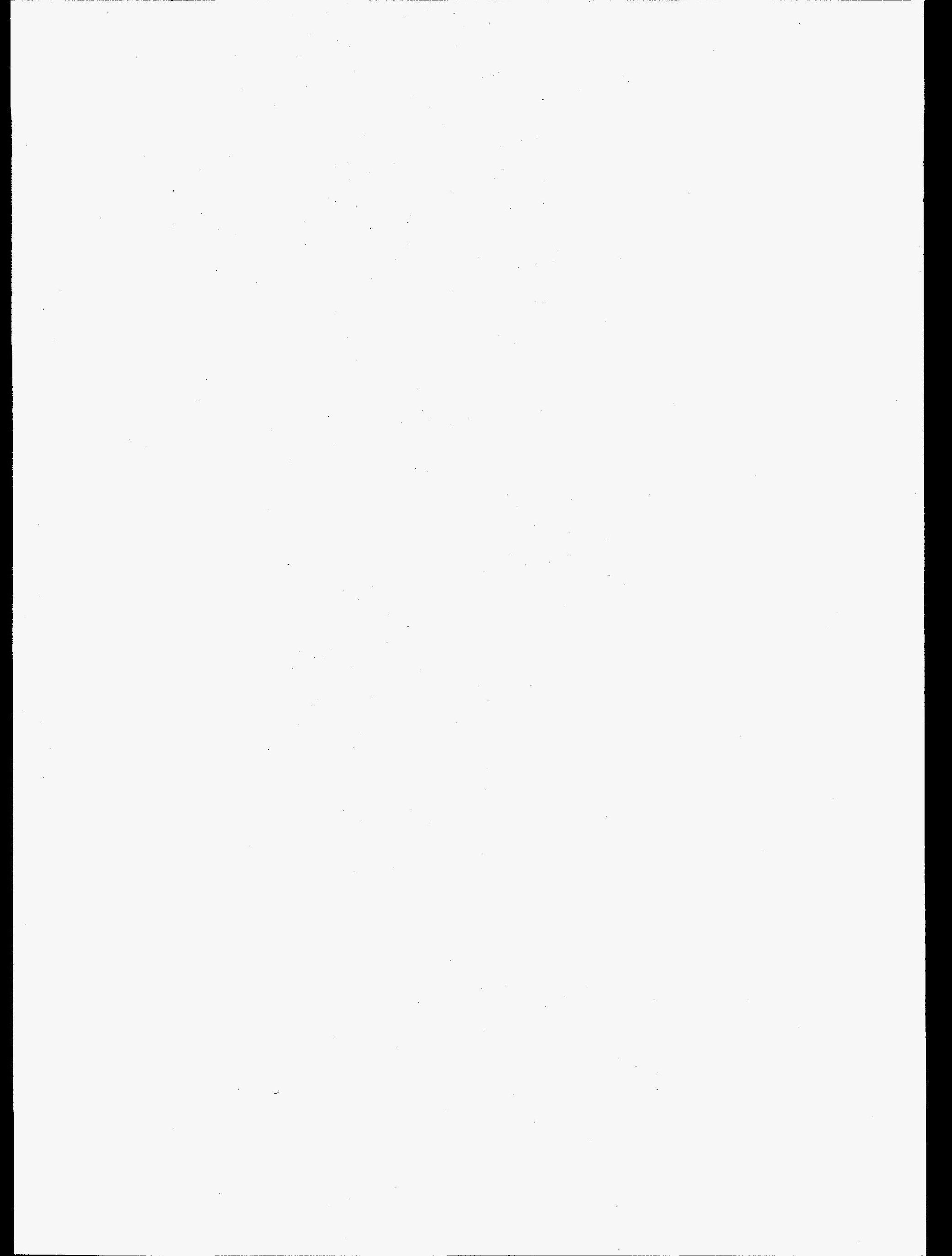


Power Burst Facility 1994 Detail Data

Power Burst Facility (PBF) Bar Graphs of Annual Data by Month . . . . . . . . INEL-149 



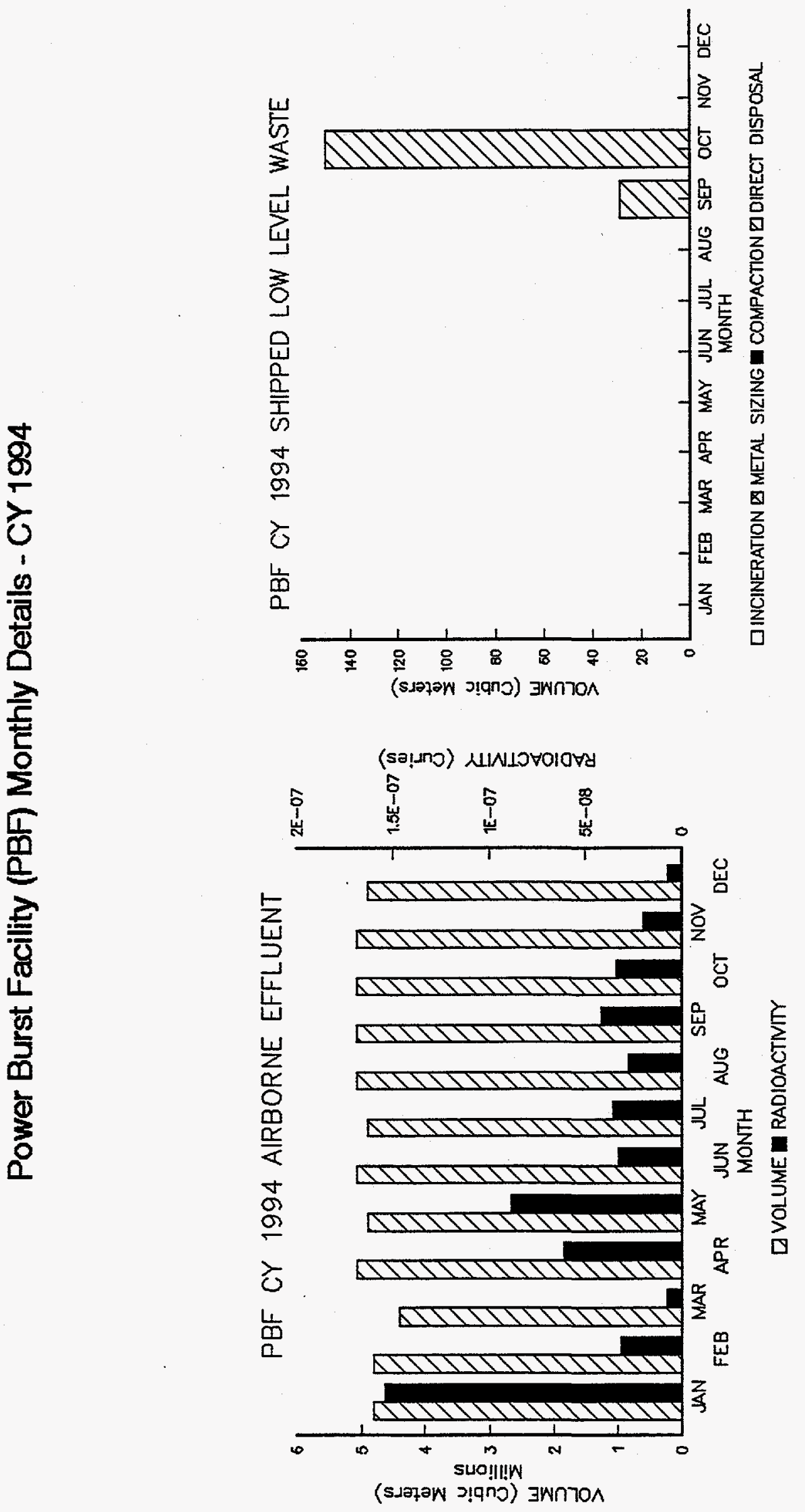

INEL-149 

Special Manufacturing Capability 1994 Detail Data

Special Manufacturing Capability (SMC) Bar Graphs of Annual Data by Month

INEL-153 



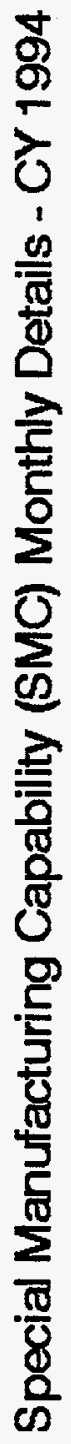
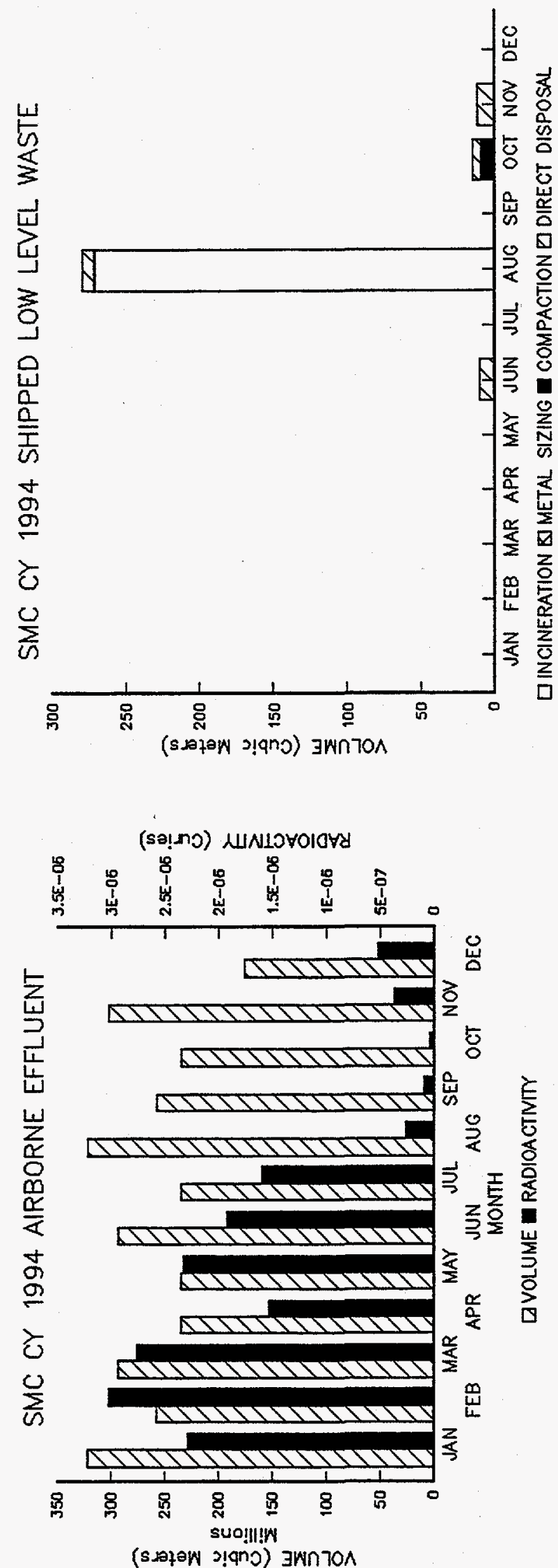

INEL-153 


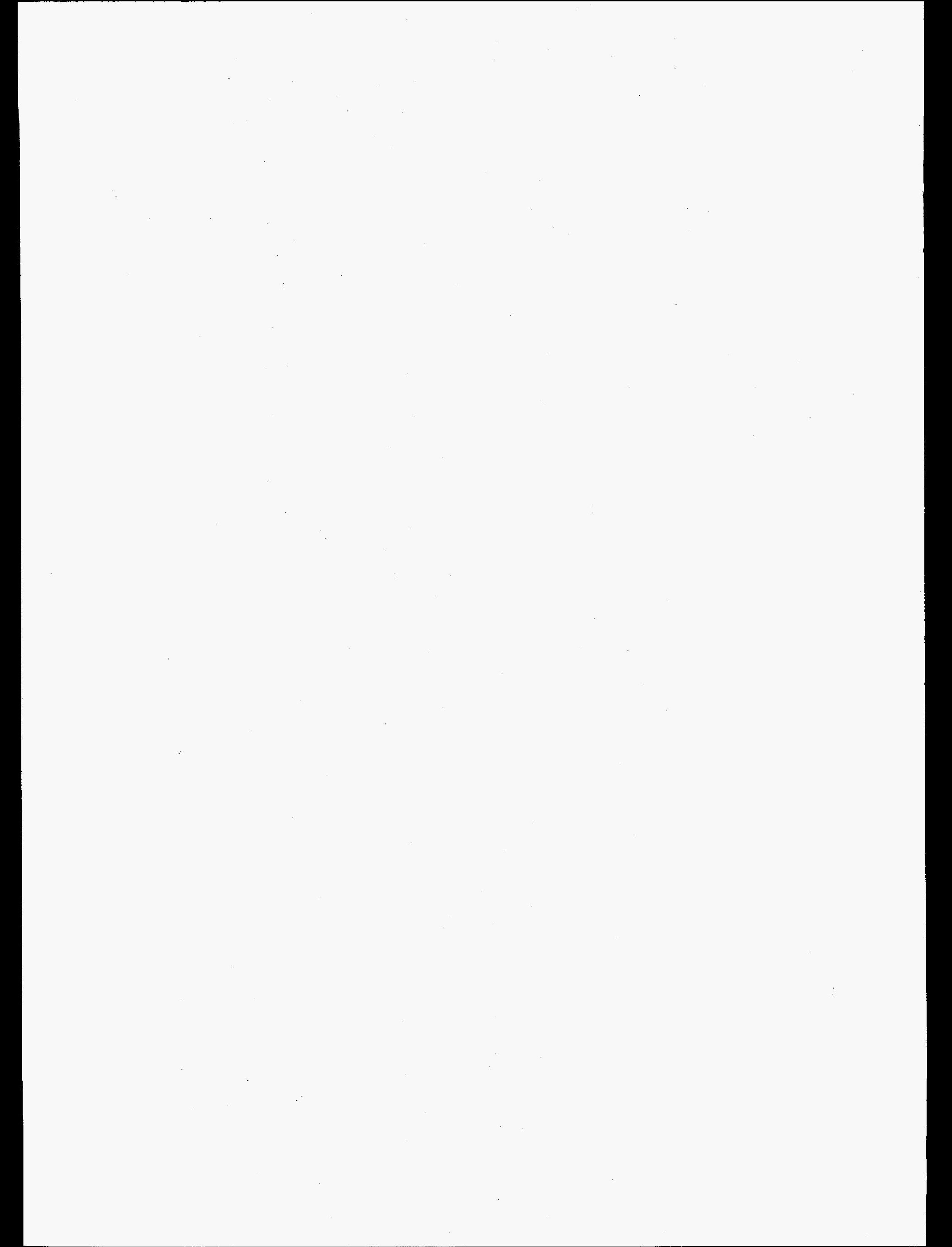


Test Area North 1994 Detail Data

Test Area North (TAN) Bar Graphs of Annual Data by Month $\ldots \ldots \ldots \ldots$ INEL-157 


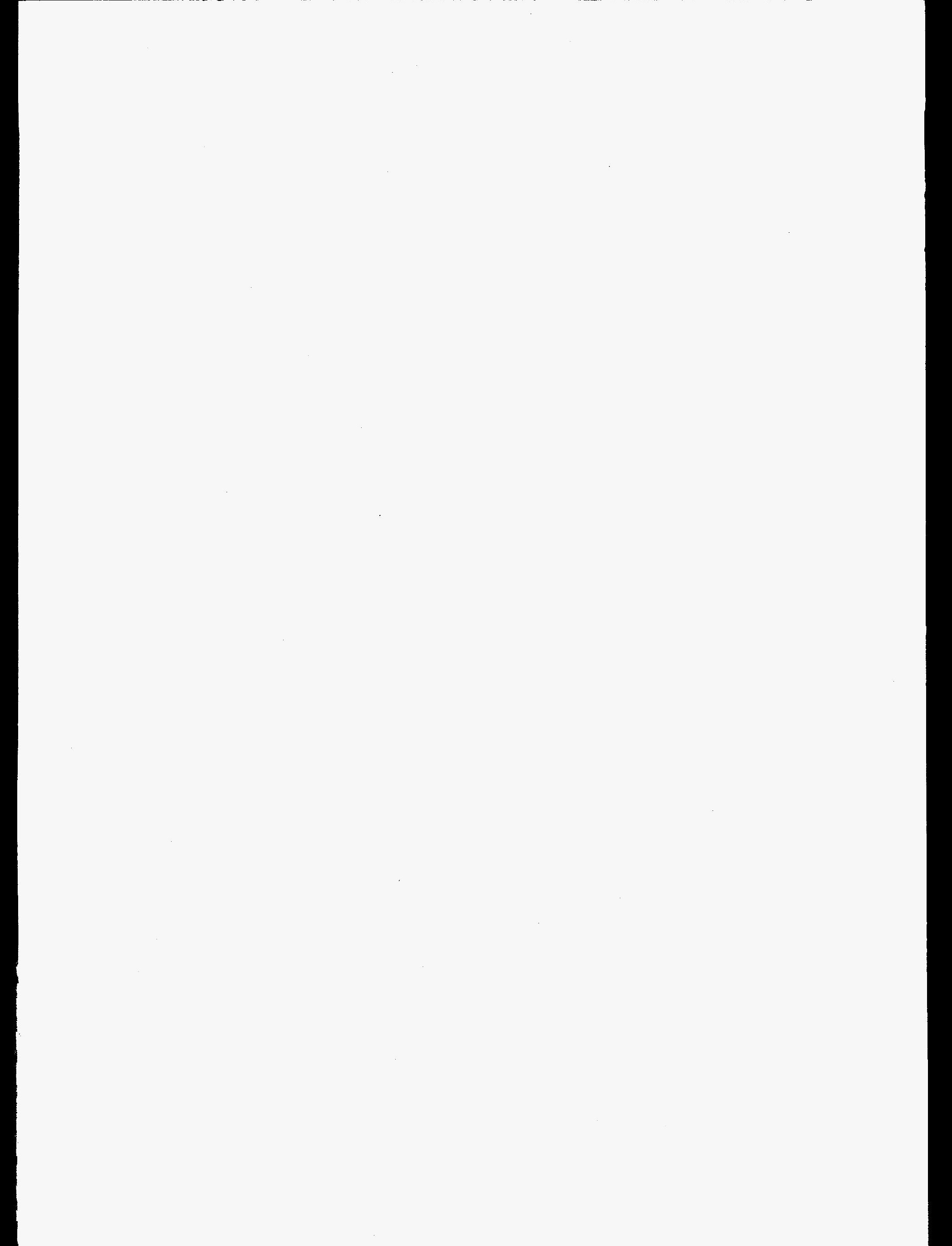




\section{Test Area North (TAN) Monthly Details - CY 1994}
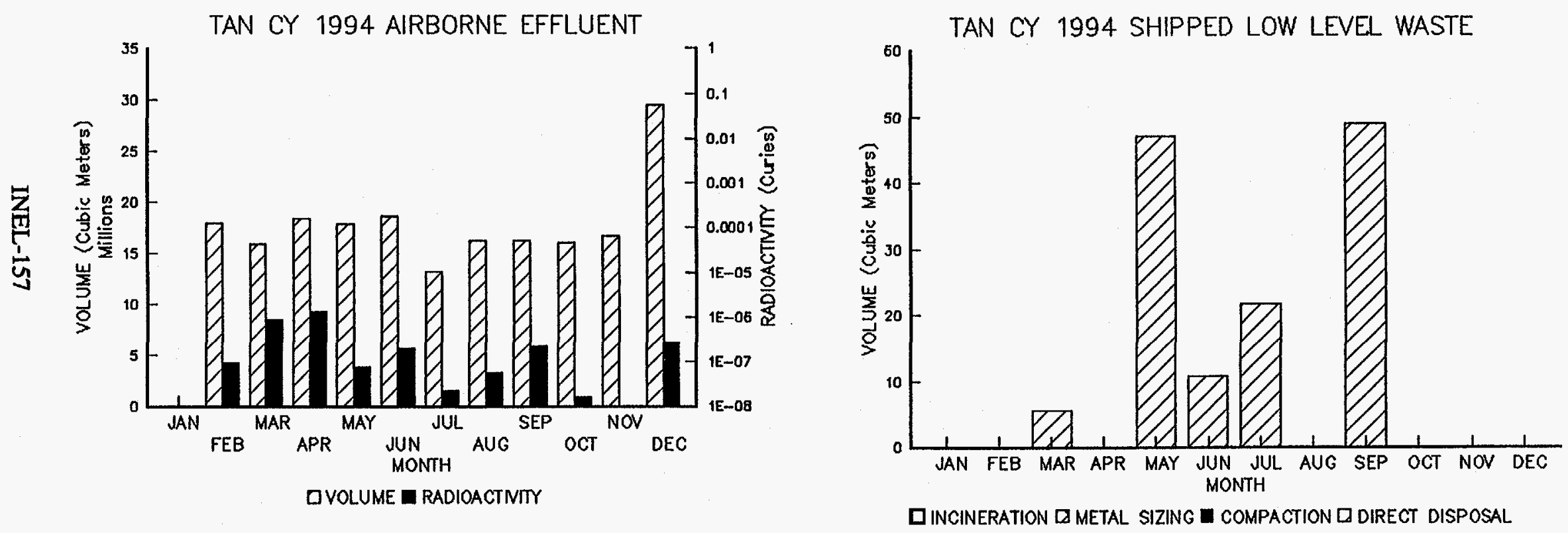
Test Reactor Area 1994 Detail Data

Test Reactor Area (TRA) Bar Graphs of Annual Data by Month . . . . . . . . INEL-161 


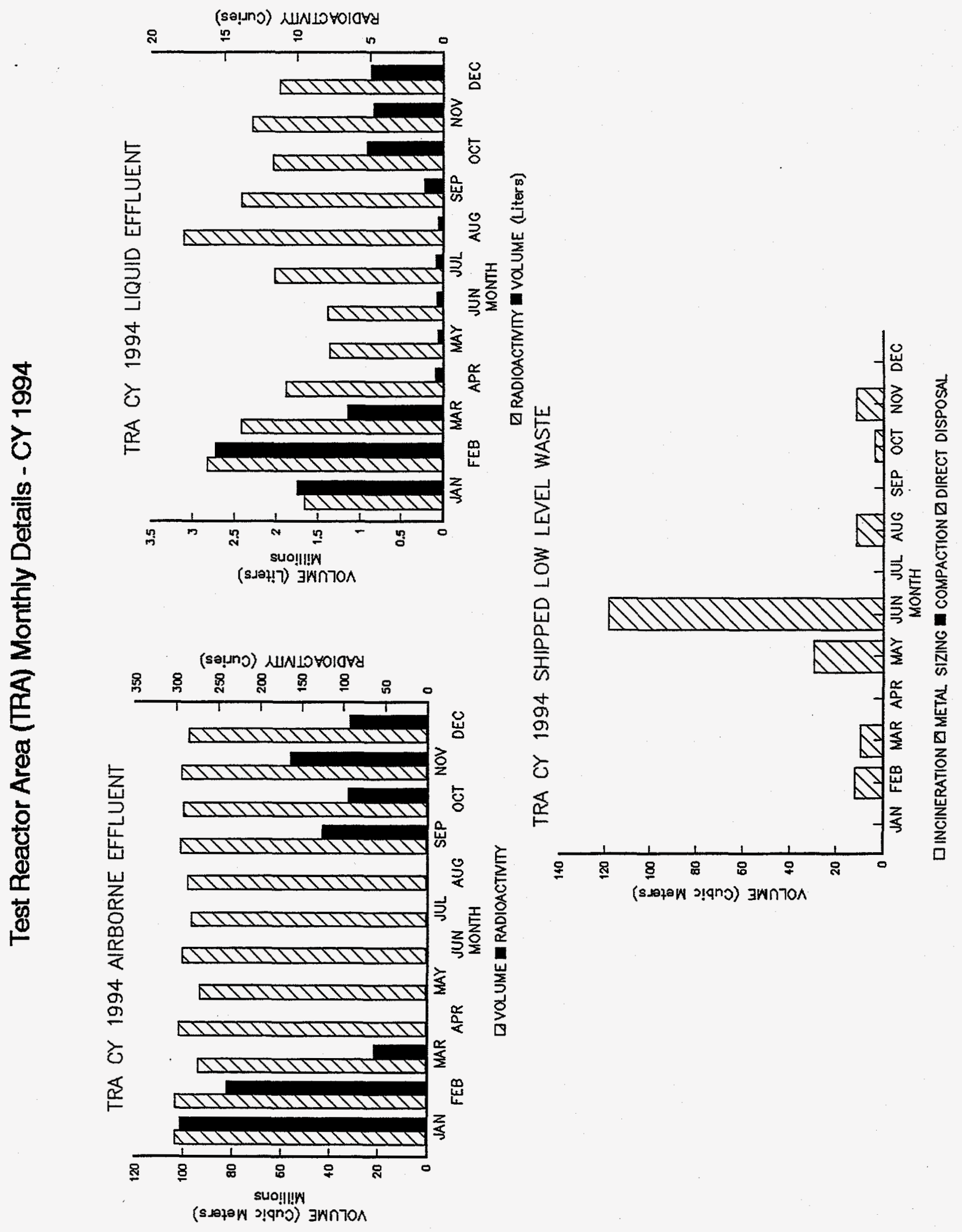




\section{Test Reactor Area (TRA) Monthly Details - CY 1994}
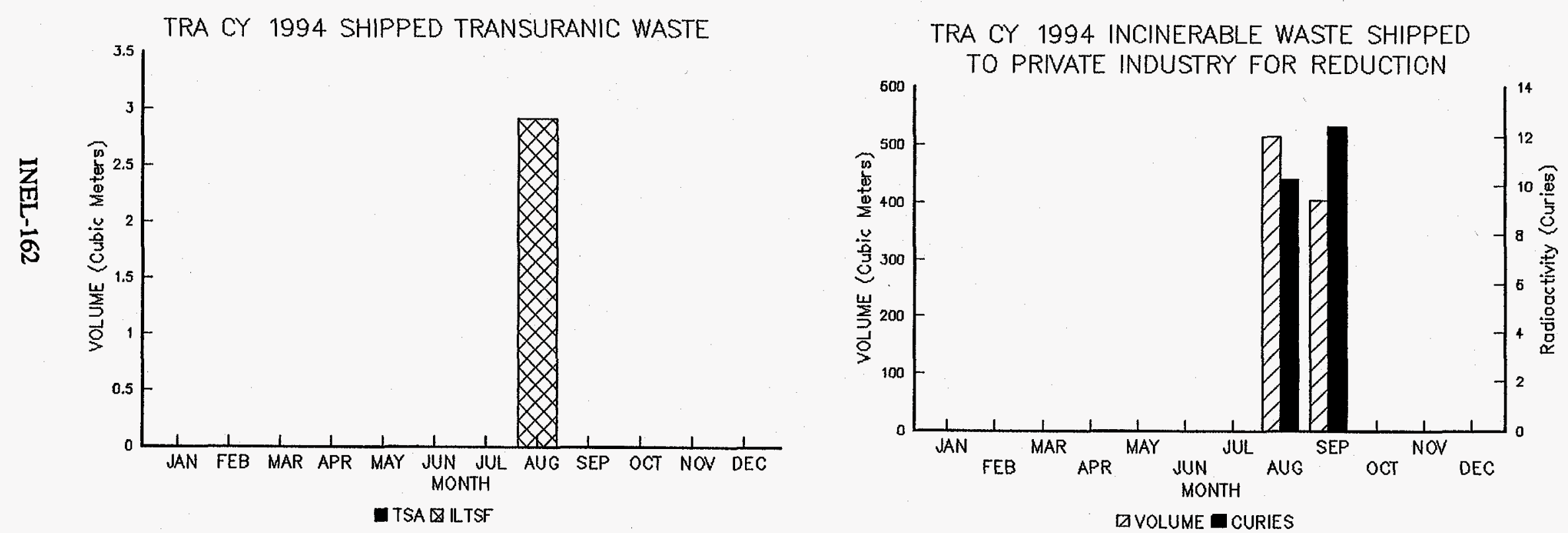
Waste Experimental Reduction Facility 1994 Detail Data

Waste Experimental Reduction Facility (WER) Bar Graphs

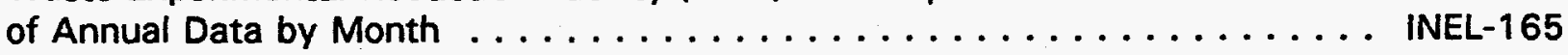





\section{Waste Experinmental Reduction Facility (WER) Monthly Details - CY 1994}
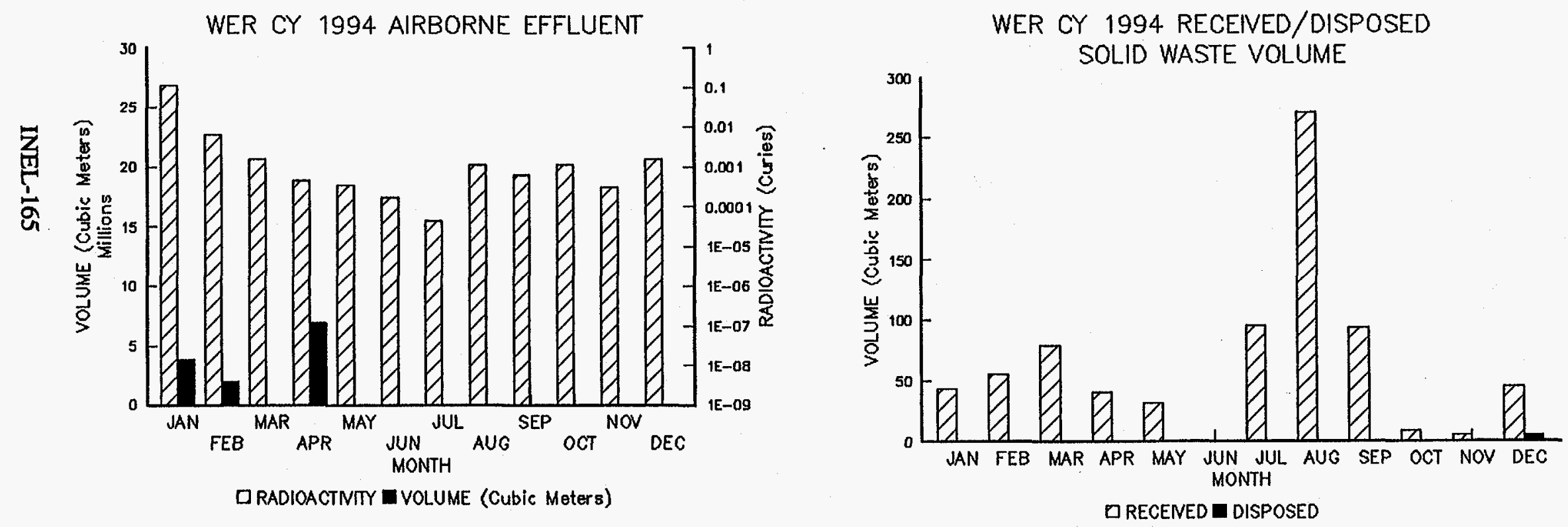


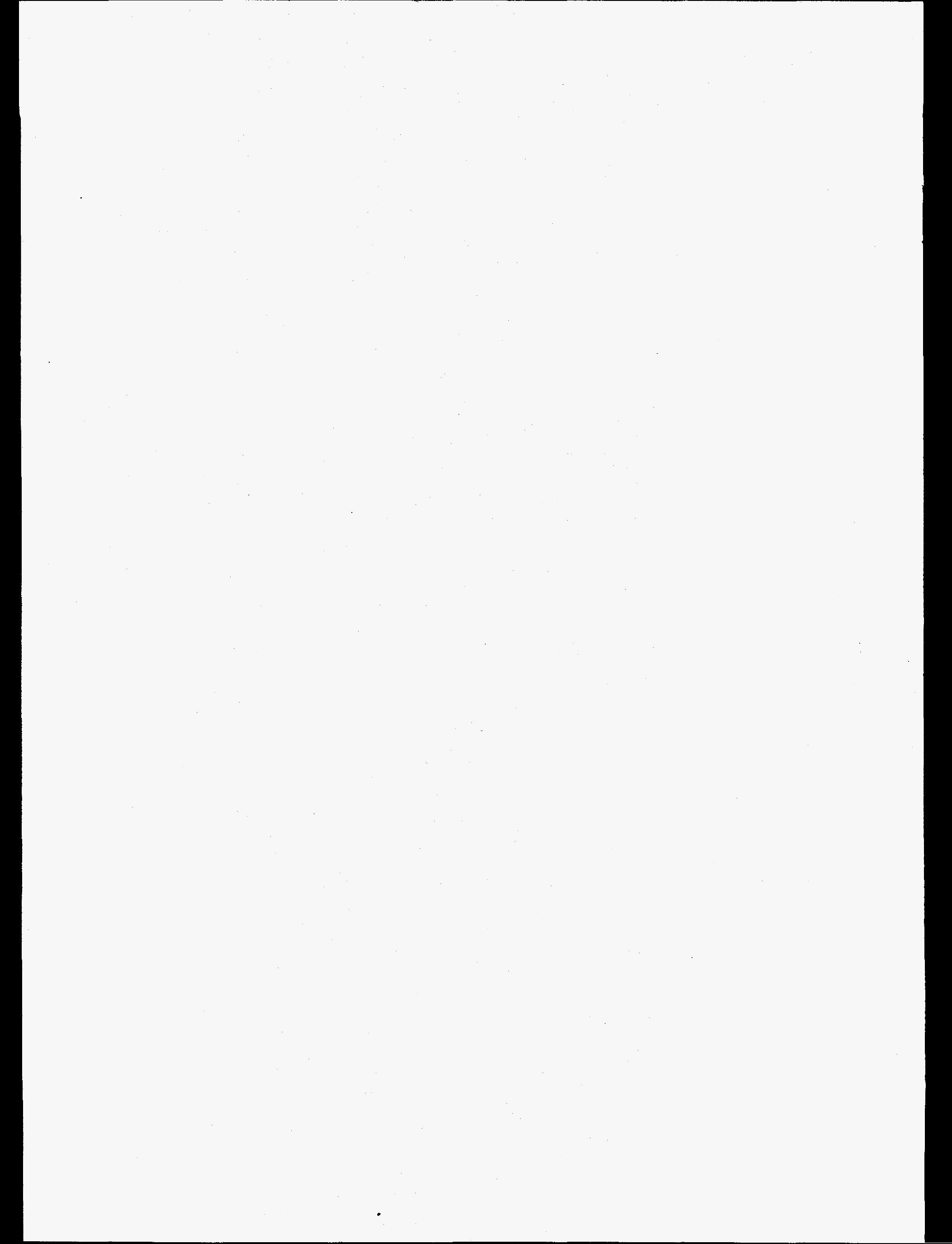




\section{Engineered Release Points}



Table 1. Engineered release points to the environment.

\begin{tabular}{|c|c|c|c|c|}
\hline $\begin{array}{l}\text { Type of } \\
\text { Discharge }\end{array}$ & Area/Location & $\begin{array}{l}\text { Type of Description of } \\
\text { Discharge Surface }\end{array}$ & $\begin{array}{l}\text { Air Emission } \\
\text { Inventory } \\
\text { Stack No. }\end{array}$ & $\begin{array}{c}\text { Active } \\
\text { (Yes/No) }\end{array}$ \\
\hline \multirow[t]{15}{*}{ Airborne } & $\begin{array}{l}\text { ANL/EBR-II, } \\
\text { FCF }\end{array}$ & $\begin{array}{l}61-\mathrm{m} \text { glass-coated steel stack, } 30 \mathrm{~m}^{3} / \mathrm{s} \text { discharge } \\
\text { capacity, continuously monitored. }\end{array}$ & ANL-764-001 & Yes \\
\hline & ANL/FASB & $\begin{array}{l}10-\mathrm{m} \text { stack, } 6.64 \mathrm{~m}^{3} / \mathrm{s} \text { discharge capacity, } \\
\text { continuously monitored. }\end{array}$ & ANL-787-001 & Yes \\
\hline & ANL/FMF & $\begin{array}{l}11.1-\mathrm{m} \text { stack, } 4.6 \mathrm{~m}^{3} / \mathrm{s} \text { discharge capacity, } \\
\text { continuously monitored. }\end{array}$ & ANL-704-008 & Yes \\
\hline & ANL/HFEF & $\begin{array}{l}28.6-\mathrm{m} \text { stack, } 21.7 \mathrm{~m}^{3} / \mathrm{s} \text { discharge capacity, } \\
\text { continuously monitored. }\end{array}$ & ANL-785-018 & Yes \\
\hline & ANL/L\&O & $\begin{array}{l}\text { Two separate stacks-one stack is } 15.2 \mathrm{~m} \text { with } \\
10.9 \mathrm{~m}^{3} / \mathrm{s} \text { discharge capacity. The other stack is } \\
11.3 \mathrm{~m} \text { with } 7.8 \mathrm{~m}^{3} / \mathrm{s} \text { discharge capacity. Both } \\
\text { stacks are continuously monitored. }\end{array}$ & $\begin{array}{l}\text { ANL-752-004 } \\
\text { (MAIN) } \\
\text { ANL-752-005 } \\
\quad \text { (NDA) }\end{array}$ & Yes \\
\hline & ANL/RLWTF & $\begin{array}{l}\text { 14.2-m stack, } 1.7 \mathrm{~m}^{3} / \mathrm{s} \text { discharge capacity, } \\
\text { continuously monitored. }\end{array}$ & ANL-798-017 & Yes \\
\hline & ANL/SCMS & $\begin{array}{l}14.6-\mathrm{m} \text { stack, } 4.7 \mathrm{~m}^{3} / \mathrm{s} \text { discharge capacity, } \\
\text { continuously monitored. }\end{array}$ & ANL-793-001 & Yes \\
\hline & ANL/SPF & $\begin{array}{l}\text { 6.9-m stack, } 2.1 \mathrm{~m}^{3} / \mathrm{s} \text { discharge capacity, } \\
\text { continuously monitored. This stack is presently } \\
\text { inactive. }\end{array}$ & ANL-799-010 & No \\
\hline & ANL/TREAT & $\begin{array}{l}42.7-\mathrm{m} \text { steel stack, } 2.8 \mathrm{~m}^{3} / \mathrm{s} \text { discharge capacity, } \\
\text { continuously monitored. }\end{array}$ & ANL-720-007 & Yes \\
\hline & ANL/ZPPR & $\begin{array}{l}22.9-\mathrm{m} \text { steel stack, } 2.3 \mathrm{~m}^{3} / \mathrm{s} \text { discharge capacity, } \\
\text { continuously monitored. }\end{array}$ & ANL-777-002 & Yes \\
\hline & ARA & $\begin{array}{l}9.1-\mathrm{m} \text { stack, } 1.4 \mathrm{~m}^{3} / \mathrm{s} \text { discharge capacity, } \\
\text { continuously monitored. These stacks were } \\
\text { capped in } 1989 . \text { D\&D removal scheduled for } \\
1996 .\end{array}$ & ARA001 & No \\
\hline & CFA/West & $\begin{array}{l}\text { Two normal ventilation exhausts, respirator } \\
\text { maintenance } 0.6 \mathrm{~m}^{3} / \mathrm{s} \text { flow, sampled weekly } \\
\text { when operating. Facility placed on standby } \\
\text { June } 1,1993 \text {. }\end{array}$ & $\begin{array}{l}\text { Drying ovens } \\
\text { CFA-617-030 } \\
\text { CFA-617-031 }\end{array}$ & No \\
\hline & CFA/North & $\begin{array}{l}\text { Normal ventilation exhausts, laundry dryer, } \\
6.1 \mathrm{~m}^{3} / \mathrm{s} \text { flow, continuously sampled when } \\
\text { operating. }\end{array}$ & $\begin{array}{l}\text { CFA-617-010 } \\
\text { CFA-617-011 }\end{array}$ & No \\
\hline & CPP/FAST & $\begin{array}{l}50-\mathrm{m} \text { stack, } 66.1 \mathrm{~m}^{3} / \mathrm{s} \text { discharge capacity, } \\
\text { continuously monitored and sampled for } \\
\text { particulates only. }\end{array}$ & CPP-767-001 & Yes \\
\hline & $\begin{array}{l}\text { CPP/Main } \\
\text { Stack }\end{array}$ & $\begin{array}{l}76.2-\mathrm{m} \text { stack, } 84.95 \mathrm{~m}^{3} / \mathrm{s} \text { discharge capacity, } \\
\text { continuously monitored. }\end{array}$ & CPP-708-001 & Yes \\
\hline
\end{tabular}


Table 1. (continued.)

\begin{tabular}{|c|c|c|c|c|}
\hline $\begin{array}{l}\text { Type of } \\
\text { Discharge }\end{array}$ & Area/Location & $\begin{array}{l}\text { Type of Description of } \\
\text { Discharge Surface }\end{array}$ & $\begin{array}{l}\text { Air Emission } \\
\text { Inventory } \\
\text { Stack No. }\end{array}$ & $\begin{array}{c}\text { Active } \\
\text { (Yes/No) }\end{array}$ \\
\hline & CPP/NWCF & $\begin{array}{l}22-\mathrm{m} \text { stack, } 51.9 \mathrm{~m}^{3} / \mathrm{s} \text { discharge capacity, } \\
\text { continuously monitored and sampled. }\end{array}$ & CPP-659-033 & Yes \\
\hline & $\begin{array}{l}\text { CPP/RAL } \\
\text { Stack }\end{array}$ & $\begin{array}{l}14.8-\mathrm{m} \text { stack, } 8.5 \mathrm{~m}^{3} / \mathrm{s} \text { discharge capacity, } \\
\text { continuously monitored and sampled. }\end{array}$ & CPP-684-001 & Yes \\
\hline & $\begin{array}{l}\text { CTF } \\
\text { (formerly LOF) }\end{array}$ & $\begin{array}{l}45.72-\mathrm{m} \text { stack, } 7.8 \mathrm{~m}^{3} / \mathrm{s} \text { discharge capacity, } \\
\text { continuously monitored. This facility is not } \\
\text { functioning and has no future plans to change } \\
\text { status. }\end{array}$ & TAN-725-001 & No \\
\hline & \multirow[t]{5}{*}{ NRF/A1W } & $\begin{array}{l}13.7 \text {-m stack, } .47 \mathrm{~m}^{3} / \mathrm{s} \text { discharge capacity, } \\
\text { continuously monitored. }\end{array}$ & NRF-617-013 & Yes \\
\hline & & $\begin{array}{l}13.7-\mathrm{m} \text { stack, } 4.7 \mathrm{~m}^{3} / \mathrm{s} \mathrm{A-RC} 4.7 \mathrm{~m}^{3} / \mathrm{s}, \mathrm{B}-\mathrm{RC} \text {; } \\
\text { these two share a stack, continuously monitored } \\
\text { while operating. }\end{array}$ & $\begin{array}{l}\text { NRF-617-020 } \\
\text { NRF-617-021 }\end{array}$ & $\begin{array}{l}\text { Yes } \\
\text { Yes }\end{array}$ \\
\hline & & $\begin{array}{l}\text { 12.2-m stack, } 8.9 \mathrm{~m}^{3} / \mathrm{s} \text { discharge capacity, } \\
\text { continuously monitored while operating. }\end{array}$ & NRF-616-012 & Yes \\
\hline & & $\begin{array}{l}9.14-\mathrm{m} \text { stack, } 39.6 \mathrm{~m}^{3} / \mathrm{s} \text { ELT, infrequent } \\
\text { operation. }\end{array}$ & NRF-616-039 & Yes \\
\hline & & $\begin{array}{l}17.1-\mathrm{m} \text { stack, } 17.1 \mathrm{~m}^{3} / \mathrm{s} \text { emergency } \mathrm{RC} \text {, } \\
\text { infrequent operation. }\end{array}$ & NRF-616A-002 & Yes \\
\hline & $\begin{array}{l}\text { NRF/AlW- } \\
\text { RWDS }\end{array}$ & $\begin{array}{l}6.7-\mathrm{m} \text { stack, } 283 \mathrm{~m}^{3} / \mathrm{s} \mathrm{RWDE} \text {, infrequent } \\
\text { operation. }\end{array}$ & NRF-628-006 & Yes \\
\hline & \multirow[t]{3}{*}{ NRF/ECF } & $\begin{array}{l}18 \text { High bay roof vents, } 20.4-\mathrm{m} \text { vents, } \\
13900 \mathrm{~m}^{3} / \mathrm{s} \text { continuously monitored. }\end{array}$ & $\begin{array}{c}\text { NRF-618-024 } \\
\text { through } \\
\text { NRF-618-029 } \\
\text { NRF-618-032 } \\
\text { through } \\
\text { NRF-618-043 }\end{array}$ & Yes \\
\hline & & $\begin{array}{l}31.1-\mathrm{m} \text { stack, } 23 \mathrm{~m}^{3} / \mathrm{s} ; \text { Stack } 1 \text { continuously } \\
\text { monitored. }\end{array}$ & NRF-618-099 & Yes \\
\hline & & $\begin{array}{l}18-\mathrm{m} \text { stack, } 12 \mathrm{~m}^{3} / \mathrm{s} ; \text { Stack } 2 \text { continuously } \\
\text { monitored. }\end{array}$ & NRF-618-103 & Yes \\
\hline & \multirow[t]{3}{*}{ NRF/S1W } & $45-m$ stack, $2.85 \mathrm{~m}^{3} / \mathrm{s}$, fan room & NRF-601-019C & Yes \\
\hline & & $\begin{array}{l}1.3 \mathrm{~m}^{3} / \mathrm{s} \text { Chem stack shares the stack but } \\
\text { continuously monitored separately. }\end{array}$ & NRF-601-019A & Yes \\
\hline & & $\begin{array}{l}16-\mathrm{m} \text { stack, } 1 \mathrm{~m}^{3} / \mathrm{s} \mathrm{RC} \text { exhaust; infrequent } \\
\text { operation, monitored while operating. }\end{array}$ & NRF-601-023 & Yes \\
\hline & NRF/S5G & $\begin{array}{l}26-\mathrm{m} \text { stack, } 12.5 \mathrm{~m}^{3} / \mathrm{s} \text { RAVE; continuously } \\
\text { operating, continuously monitored. }\end{array}$ & NRF-633A-057 & Yes \\
\hline
\end{tabular}


Table 1. (continued.)

\begin{tabular}{|c|c|c|c|c|}
\hline $\begin{array}{l}\text { Type of } \\
\text { Discharge }\end{array}$ & Area/Location & $\begin{array}{l}\text { Type of Description of } \\
\text { Discharge Surface }\end{array}$ & $\begin{array}{l}\text { Air Emission } \\
\text { Inventory } \\
\text { Stack No. }\end{array}$ & $\begin{array}{c}\text { Active } \\
\text { (Yes/No) }\end{array}$ \\
\hline & NRF/MSC & $\begin{array}{l}\text { Miscellaneous and fugitive sources, ground } \\
\text { level. }\end{array}$ & & Yes \\
\hline & PBF & $\begin{array}{l}\text { 24.4-m stack, } 2.8 \mathrm{~m}^{3} / \mathrm{s} \text { discharge capacity, } \\
\text { continuously monitored. }\end{array}$ & $\begin{array}{l}\text { PER } 620 \\
\text { Vent } 016\end{array}$ & Yes \\
\hline & $\begin{array}{l}\text { SMC/R\&D } \\
\text { Process }\end{array}$ & $\begin{array}{l}17.7-\mathrm{m} \text { stack, } 23.6 \mathrm{~m}^{3} / \mathrm{s} \text { discharge capacity, } \\
\text { continuously monitored. }\end{array}$ & TAN-607-039 & Yes \\
\hline & $\begin{array}{l}\text { SMC/MDF QC } \\
\text { LAB }\end{array}$ & $\begin{array}{l}17.99-\mathrm{m} \text { stack, } 7.79 \mathrm{~m}^{3} / \mathrm{s} \text { discharge capacity, } \\
\text { continuously monitored. }\end{array}$ & TAN-607-119 & Yes \\
\hline & SMC/LINE 2a & $\begin{array}{l}\text { 14.4-m stack, } 18.12 \mathrm{~m}^{3} / \mathrm{s} \text { discharge capacity, } \\
\text { continuously monitored. }\end{array}$ & TAN-629-013 & Yes \\
\hline & SMC/LINE $2 b$ & $\begin{array}{l}8.36-\mathrm{m} \text { stack, } 3.12 \mathrm{~m}^{3} / \mathrm{s} \text { discharge capacity, } \\
\text { continuously monitored. }\end{array}$ & TAN-629-014 & Yes \\
\hline & SMC/LINE 2B & $\begin{array}{l}8.36-\mathrm{m} \text { stack, } 3.12 \mathrm{~m}^{3} / \mathrm{s} \text { discharge capacity, } \\
\text { continuously monitored. }\end{array}$ & TAN-629-012 & Yes \\
\hline & $\begin{array}{l}\text { SMC/S. } \\
\text { Process S6 }\end{array}$ & $\begin{array}{l}16.97-\mathrm{m} \text { stack, } 7.08 \mathrm{~m}^{3} / \mathrm{s} \text { discharge capacity, } \\
\text { continuously monitored. }\end{array}$ & TAN-679-027 & Yes \\
\hline & $\begin{array}{l}\text { SMC/S. } \\
\text { Process S7 }\end{array}$ & $\begin{array}{l}16.97-\mathrm{m} \text { stack, } 7.08 \mathrm{~m}^{3} / \mathrm{s} \text { discharge capacity, } \\
\text { continuously monitored. }\end{array}$ & TAN-679-026 & Yes \\
\hline & $\begin{array}{l}\text { SMC/S. } \\
\text { Process S8 }\end{array}$ & $\begin{array}{l}16.97-\mathrm{m} \text { stack, } 7.08 \mathrm{~m}^{3} / \mathrm{s} \text { discharge capacity, } \\
\text { continuously monitored. }\end{array}$ & TAN-679-025 & Yes \\
\hline & $\begin{array}{l}\text { SMC/S. } \\
\text { Process } \$ 9\end{array}$ & $\begin{array}{l}16.97-\mathrm{m} \text { stack, } 7.08 \mathrm{~m}^{3} / \mathrm{s} \text { discharge capacity, } \\
\text { continuously monitored. }\end{array}$ & TAN-679-024 & Yes \\
\hline & $\begin{array}{l}\text { SMC/S. } \\
\text { Process S10 }\end{array}$ & $\begin{array}{l}16.97-\mathrm{m} \text { stack, } 7.08 \mathrm{~m}^{3} / \mathrm{s} \text { discharge capacity, } \\
\text { continuously monitored. }\end{array}$ & TAN-679-023 & Yes \\
\hline & $\begin{array}{l}\text { SMC/S. } \\
\text { Process S11 }\end{array}$ & $\begin{array}{l}16.97-\mathrm{m} \text { stack, } 7.08 \mathrm{~m}^{3} / \mathrm{s} \text { discharge capacity, } \\
\text { continuously monitored. }\end{array}$ & TAN-679-022 & Yes \\
\hline & $\begin{array}{l}\text { SMC/Scrap } \\
\text { Handling }\end{array}$ & $\begin{array}{l}16.97-\mathrm{m} \text { stack, } 7.93 \mathrm{~m}^{3} / \mathrm{s} \text { discharge capacity, } \\
\text { continuously monitored. }\end{array}$ & TAN-681-020 & Yes \\
\hline & $\begin{array}{l}\text { SMC/Liquid } \\
\text { Reclaim }\end{array}$ & $\begin{array}{l}16.97-\mathrm{m} \text { stack, } 7.93 \mathrm{~m}^{3} / \mathrm{s} \text { discharge capacity, } \\
\text { continuously monitored. }\end{array}$ & TAN-681-018 & Yes \\
\hline & $\begin{array}{l}\text { SMC/PRF } \\
\text { Stack S14 }\end{array}$ & $\begin{array}{l}\text { 33.54-m stack, } 0.94 \mathrm{~m}^{3} / \mathrm{s} \text { discharge capacity, } \\
\text { continuously monitored. }\end{array}$ & TAN-681-012 & Yes \\
\hline & TAN Decon & $\begin{array}{l}29.6-\mathrm{m} \mathrm{stack}, 24.9 \mathrm{~m}^{3} / \mathrm{s} \text { discharge capacity, } \\
\text { continuously monitored currently no emissions. } \\
\text { Currently in process of deactivation. }\end{array}$ & TAN-607-136 & No \\
\hline & TAN/TSF & $\begin{array}{l}\text { 51.4-m stack, } 7.9 \mathrm{~m}^{3} / \mathrm{s} \text { discharge capacity, } \\
\text { continuously monitored. }\end{array}$ & TAN-734-001 & Yes \\
\hline
\end{tabular}


Table 1. (continued.)

\begin{tabular}{|c|c|c|c|c|}
\hline $\begin{array}{c}\text { Type of } \\
\text { Discharge } \\
\end{array}$ & Area/Location & $\begin{array}{c}\text { Type of Description of } \\
\text { Discharge Surface }\end{array}$ & $\begin{array}{l}\text { Air Emission } \\
\text { Inventory } \\
\text { Stack No. } \\
\end{array}$ & $\begin{array}{c}\text { Active } \\
\text { (Yes/No) } \\
\end{array}$ \\
\hline & TAN/PREPP & $\begin{array}{l}26.1-\mathrm{m} \text { stack, } 3 \mathrm{~m}^{3} / \mathrm{s} \text { discharge capacity, } \\
\text { continuously monitored. Facility was never } \\
\text { activated-currently being scheduled for } \\
\text { decommissioning. }\end{array}$ & TAN-607-107 & No \\
\hline & TRA/ATR & $\begin{array}{l}76.2-\mathrm{m} \text { stack, } 21.2 \mathrm{~m}^{3} / \mathrm{s} \text { discharge capacity, } \\
\text { continuously monitored. }\end{array}$ & TRA-770-001 & Yes \\
\hline & $\begin{array}{l}\text { TRA/Chem } \\
\text { Lab }\end{array}$ & $\begin{array}{l}29.6-\mathrm{m} \text { stack, } 6.6 \mathrm{~m}^{3} / \mathrm{s} \text { discharge capacity, } \\
\text { continuously monitored. }\end{array}$ & TRA-604-035 & Yes \\
\hline & TRA/ETR & $\begin{array}{l}76.2-\mathrm{m} \text { stack, } 9.4 \mathrm{~m}^{3} / \mathrm{s} \text { discharge capacity, } \\
\text { continuously monitored. As of March } 1988 \text {, this } \\
\text { stack is not monitored. No activity is } \\
\text { discharged. }\end{array}$ & TRA-753-001 & Yes \\
\hline & TRA/Hot Cells & $\begin{array}{l}15.2-\mathrm{m} \text { stack, } 1.6 \mathrm{~m}^{3} / \mathrm{s} \text { discharge capacity, } \\
\text { continuously monitored. Three stacks sampled } \\
\text { as one. }\end{array}$ & TRA-632-030 & Yes \\
\hline & TRA/MTR & $\begin{array}{l}76.2 \text {-m stack, } 6 \mathrm{~m}^{3} / \mathrm{s} \text { discharge capacity, } \\
\text { continuously monitored. }\end{array}$ & TRA-710-001 & Yes \\
\hline & TRA/661 & $\begin{array}{l}8.43-\mathrm{m} \text { stack, } 3.3 \mathrm{~m}^{3} / \mathrm{s} \text { discharge capacity, not } \\
\text { monitored. }\end{array}$ & TRA-661-008 & Yes \\
\hline & WER/North & $\begin{array}{l}15-\mathrm{m} \text { stack, } 8 \mathrm{~m}^{3} / \mathrm{s} \text { discharge capacity, } \\
\text { continuously monitored. }\end{array}$ & PER-755-001 & Yes \\
\hline & WER/South & $\begin{array}{l}15-\mathrm{m} \text { stack, } 9.4 \mathrm{~m}^{3} / \mathrm{s} \text { discharge capacity, } \\
\text { continuously monitored. }\end{array}$ & PER-756-001 & Yes \\
\hline & WER/765 & $\begin{array}{l}15.5-\mathrm{m} \text { stack, } 4.7 \mathrm{~m}^{3} / \mathrm{s} \text { discharge capacity, } \\
\text { continuously monitored. }\end{array}$ & PER-765-001 & Yes \\
\hline & WMC/SWEPP & $\begin{array}{l}4.5-\mathrm{m} \text { stack, } 1.7 \mathrm{~m}^{3} / \mathrm{s} \text { discharge capacity, } \\
\text { continuously monitored during active Drum Vent } \\
\text { operation. }\end{array}$ & WMF-615-001 & Yes \\
\hline $\begin{array}{l}\text { Liquid } \\
\text { (injection } \\
\text { well) }\end{array}$ & CPP & $\begin{array}{l}181 \mathrm{~m} \text { deep ( } 42.7 \mathrm{~m} \text { below water table), } \\
\text { constantly monitored by radiation detector with a } \\
\text { detection unit of } 2 \times 10^{-6} \mathrm{Ci} / \mathrm{mL} \text {, flow } \\
\text { approximately } 170 \times 10^{6} \text { liters per month. } \\
\text { (INEL discharges to the injection well have been } \\
\text { terminated since } 1986 \text {. This well was closed in } \\
\text { November 1989.) }\end{array}$ & & No \\
\hline & PBF & $\begin{array}{l}33.5 \mathrm{~m} \text { deep ( } 105 \mathrm{~m} \text { above water table), } \\
\text { constantly monitored, flow intermittent with a } \\
\text { maximum of } 750 \text { liters per minute (no longer in } \\
\text { use as of August 1980; in June 1984, this well } \\
\text { was completely filled with concrete). }\end{array}$ & & No \\
\hline
\end{tabular}


Table 1. (continued.)

\begin{tabular}{|c|c|c|c|c|}
\hline $\begin{array}{c}\text { Type of } \\
\text { Discharge }\end{array}$ & Area/Location & $\begin{array}{l}\text { Type of Description of } \\
\text { Discharge Surface }\end{array}$ & $\begin{array}{l}\text { Air Emission } \\
\text { Inventory } \\
\text { Stack No. }\end{array}$ & $\begin{array}{c}\text { Active } \\
\text { (Yes/No) }\end{array}$ \\
\hline \multirow{10}{*}{$\begin{array}{l}\text { Liquid } \\
\text { (seepage } \\
\text { ponds, } \\
\text { leaching } \\
\text { pits, cribs) }\end{array}$} & $\begin{array}{l}\text { ANL/EBR-II } \\
\text { and L\&O }\end{array}$ & $\begin{array}{l}\text { Batch monitored pond approximately } 1.2 \times 10^{4} \\
\mathrm{~m}^{2} \text { maximum depth of } 4 \mathrm{~m} \text {. }\end{array}$ & & Yes \\
\hline & ARA & $\begin{array}{l}\text { Surface depression (approximately } 1.3 \text { acre), } \\
\text { estimate flow } 1.05 \times 10^{7} \text { liters per year, } \\
\text { continuously monitored. ARA completely shut } \\
\text { down operations in September } 1986 \text {, no further } \\
\text { releases are being made to this release point } \\
\text { except for drainage as a result of rain water } \\
\text { runoff. }\end{array}$ & & No \\
\hline & CFA & $\begin{array}{l}\text { Sewage plant tile drain field, } 610 \times 61 \mathrm{~m} \text {, } \\
\text { average flow approximately } 15 \times 10^{6} \text { liters per } \\
\text { month, continuously monitored. }\end{array}$ & & Yes \\
\hline & $\begin{array}{l}\text { CPP/Pond } \\
\text { No. } 1\end{array}$ & $\begin{array}{l}\text { Percolation pond, } 412 \mathrm{ft} \times 480 \mathrm{ft} \times 16 \mathrm{ft} \text {. } \\
\text { CPP } 797 \text { is the discharge point that is } \\
\text { continuously monitored and sampled. }\end{array}$ & & Yes \\
\hline & $\begin{array}{l}\text { CPP/Pond } \\
\text { No. } 2\end{array}$ & $\begin{array}{l}\text { Percolation pond, } 498 \mathrm{ft} \times 498 \mathrm{ft} \times 12 \text { to } 14 \mathrm{ft} \text {. } \\
\text { CPP } 797 \text { is the discharge point that is } \\
\text { continuously monitored and sampled. }\end{array}$ & & Yes \\
\hline & $\begin{array}{l}\text { CTF (formerly } \\
\text { LOF) }\end{array}$ & $\begin{array}{l}\text { Continuously monitored pond approximately } \\
76 \times 152 \times \text { maximum } 5.5 \mathrm{~m} \text { deep. }\end{array}$ & & Yes \\
\hline & NRF & $\begin{array}{l}\text { Continuously monitored leaching beds handling } \\
\text { 95,000 liters per month (releases ended April } \\
\text { 1979). }\end{array}$ & & No \\
\hline & TAN & $\begin{array}{l}\text { Continuously monitored pond approximately } 13 \\
\text { hectares in size. }\end{array}$ & N/A & Yes \\
\hline & TRA & $\begin{array}{l}\text { Two ponds } 40 \times 73 \text { and one pond } 76 \times 122 \mathrm{~m} \text {, } \\
\text { average flow approximately } 90 \times 10^{6} \text { liters per } \\
\text { month, prior to } 1988 \text {. In } 1988 \text {, average flow } \\
\text { decreased to } 4.7 \times 10^{6} \text { liters per month. Ponds } \\
\text { are continuously monitored. Removed from } \\
\text { service August } 1993 \text {. }\end{array}$ & N/A & No \\
\hline & TRA & $\begin{array}{l}\text { Evaporation pond. Two adjacent lined ponds } \\
\text { measuring } 70 \mathrm{~m} \times 140 \mathrm{~m} \text { each. Double liner } \\
\text { and leak detection system. All particulate } \\
\text { activity remains in lined pond } 3 \times 10^{6} \text { liters per } \\
\text { month. Continuously monitored. Zero release } \\
\text { to soil column. }\end{array}$ & & Yes \\
\hline
\end{tabular}


Table 1. (continued.)

\begin{tabular}{lllcc}
\hline $\begin{array}{l}\text { Type of } \\
\text { Discharge }\end{array}$ & \multicolumn{1}{c}{$\begin{array}{c}\text { Type of Description of } \\
\text { Discharge Surface }\end{array}$} & $\begin{array}{c}\text { Air Emission } \\
\text { Inventory } \\
\text { Stack No. }\end{array}$ & $\begin{array}{c}\text { Active } \\
\text { (Yes/No) }\end{array}$ \\
\hline Solid & All facilities & $\begin{array}{l}\text { Disposal of radioactive solid waste accomplished } \\
\text { at the INEL Radioactive Waste Management } \\
\text { Complex (RWMC). } \\
\text { Storage of transuranic radioactive solid waste } \\
\text { accomplished at the INEL RWMC. } \\
\text { Reduction of applicable radioactive solid waste } \\
\text { volume accomplished at the INEL WERF. }\end{array}$ & N/A & Yes \\
& $\begin{array}{l}\text { Storage of calcinated solids accomplished at } \\
\text { CPP. }\end{array}$ & N/A & Yes \\
& $\begin{array}{l}\text { Storage of sodium-contaminated waste generated } \\
\text { by ANL accomplished at ANL. }\end{array}$ & N/A & Yes \\
& $\begin{array}{l}\text { Storage of mixed waste accomplished at Mixed } \\
\text { Waste Storage Facility (MWSF). }\end{array}$ & N/A & Yes \\
\hline
\end{tabular}

